\title{
Predicting the Structure and Energetics of Protein-Ligand Interaction
}

A Dissertation presented to
the Faculty of the Graduate School
at the University of Missouri
In Partial Fulfillment
of the Requirements for the Degree
Doctor of Philosophy
Zhiwei Ma
Diaoqin Zou, Dissertation Supervisor

DECEMBER 2019 
The undersigned, appointed by the Dean of the Graduate School, have examined the dissertation entitled:

Predicting the Structure and Energetics of Protein-Ligand Interaction

presented by Zhiwei Ma, a candidate for the degree of Doctor of Philosophy and hereby certify that, in their opinion, it is worthy of acceptance.

Chair: Dr. Xiaoqin Zou, Physics

Dr. Jianlin Cheng, Computer Science

Dr. Shi-jie Chen, Physics

Dr. Ioan Kosztin, Physics

Dr. Gavin M. King, Physics 


\section{DEDICATION}

This dissertation is dedicated to my parents. 


\section{ACKNOWLEDGMENTS}

First, I would like to express my deepest gratitude to my Ph.D. advisor, Dr. Xiaoqin Zou, who has mentored and supported me for my whole Ph.D. period. Dr. Zou has devoted a great deal of time and energy to help me grow from a new graduate student with few research experiences in biophysics to a researcher. Dr. Zou is my role model not only in academia but also in being a nice lady and a decent person. She is always full of energy in her work, being patient to her students and enormous enthusiasm in everyday life. I still remember that cold winter of 2014 when I arrived in Columbia, Dr. Zou supported me to get through all the hard times since then. The most encouragement to my heart is her trust and confidence in my ability. Her warmth makes this place a second home and would be carried away into a region of my dreams no matter where I am.

Second, I would like to thank my committee members, Dr. Jianlin Cheng, Dr. Shi-jie Chen, Dr. Ioan Kosztin, and Dr. Gavin M. King, for their precious time and support. During the critical discussions with me, they show me how scientists are in discerning ways of thinking. Their passion for science and their advice will constantly encourage me in the rest of my life when I am doing research.

I extend my appreciation to all my current and previous lab members, Dr. ShengYou Huang, Dr. Sam Grinter, and Benjamin Merideth, for their help and advice.

Thank Dr. Chengfei Yan and Erica Hroblak, for their great and selfless help at the time when I first arrived. I treasure and appreciate their friendship in my life. Thank Dr. Xianjin Xu, Dr. Rui Duan, and Dr. Liming Qiu for their guidance and help on my research. I also have enjoyable and fruitful collaborations with them. Thank Dr. 
Shuang Zhang and Dr. Zhe Wang for their company in the last year of my Ph.D. study. They make me enjoy simple pleasures like in the undergraduate days.

I would like to thank all my good friends in Columbia, Jialu Yan, Dr. Milica Utjesanovic, Chenhan Zhao and Yuanzhe Zhou. I am really lucky to know them in this beautiful and peaceful town here.

Last but not least, thank Dr. Bao-xing Li in Hangzhou Normal University for his inspiration. Thank my parents and my whole family for their understanding and support. 


\section{TABLE OF CONTENTS}

ACKNOWLEDGMENTS ................ ii

LIST OF TABLES $\ldots \ldots \ldots \ldots \ldots \ldots \ldots$ vii

LIST OF FIGURES $\ldots \ldots \ldots \ldots \ldots \ldots \ldots \ldots$ ix

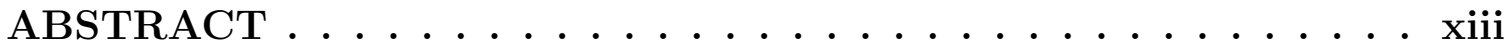

1 Introduction ...................... 1

1.1 Physical Basis of Molecular Docking . . . . . . . . . . . 4

1.1.1 Protein-ligand interaction . . . . . . . . . . . 4

1.1.2 Enthalpy-Entropy Compensation $\ldots \ldots \ldots \ldots$

1.1.3 Molecular recognition models . . . . . . . . . . . . 8

1.2 Key Components in Ligand-Protein Docking . . . . . . . . . . . . . 11

1.2 .1 Conformational sampling . . . . . . . . . . . . . . . 11

1.2 .2 Sampling algorithms . . . . . . . . . . . . . . 13

1.2 .3 Scoring functions . . . . . . . . . . . . . . . . . . 14

1.3 Docking Programs . . . . . . . . . . . . . . 16

1.4 Structure-based virtual screening and inverse virtual screening . . . . 19

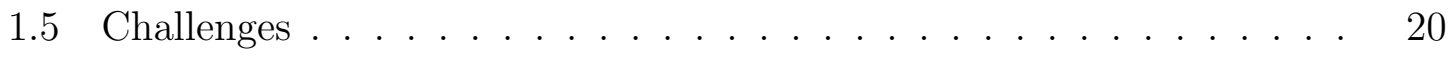

Bibliography ......................... 23

2 Drug Discovery against Diverse Kinases: Predicting Selectivity and Virtual Screening ................. 33

2.1 Introduction . . . . . . . . . . . . . . . . . . . . 34 
2.2 Materials and Methods . . . . . . . . . . . . . . 36

2.2.1 Ensemble docking algorithm to simultaneously address multiple targets . . . . . . . . . . . . . . 36

2.2 .2 Scoring function . . . . . . . . . . . . . . . 37

2.2 .3 Construction of the reference protein $\ldots \ldots \ldots$

2.2 .4 Docking algorithm $\ldots \ldots \ldots \ldots \ldots \ldots$

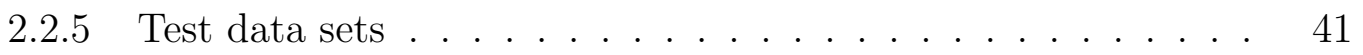

2.2 .6 Database preparation . . . . . . . . . . . . . . . . . 42

2.3 Results . . . . . . . . . . . . . . . . . . 46

2.3.1 Evaluation of the dock algorithm and scoring function . . . . 47

2.3 .2 The ligand selectivity . . . . . . . . . . . . . . . . . . . . 48

2.3.3 Virtual Database Screening _. . . . . . . . . . . . . . 53

2.3.4 Computational efficiency . . . . . . . . . . . . 55

2.4 Discussion and Conclusion . . . . . . . . . . . . . . . . 58

Bibliography ........................ 61

3 EDockMS: An Efficient Docking Platform for Multiple Target Screening ..................... 68

3.1 Introduction $\ldots \ldots \ldots \ldots \ldots \ldots \ldots \ldots \ldots \ldots \ldots$

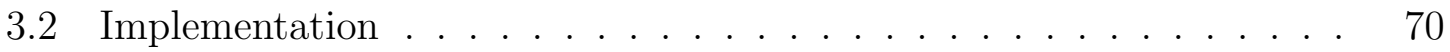

3.3 Discussion and perspectives $\ldots \ldots \ldots \ldots \ldots \ldots$

3.4 ACKNOWLEDGEMENT . . . . . . . . . . . . . 73

Bibliography ........................ 75 
4 Predicting Protein Ligand Binding Modes for CELPP and GC3:

Workflows and Insight $\ldots \ldots \ldots \ldots \ldots \ldots . \ldots \ldots$

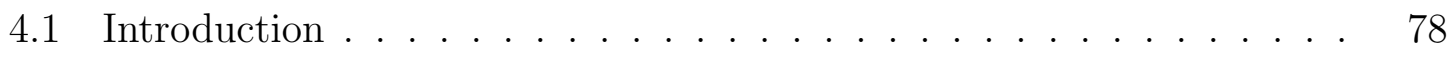

4.2 Materials and Methods . . . . . . . . . . . . . 80

4.2.1 The query protein-ligand complexes . . . . . . . . . 80

4.2.2 Overview of our binding mode prediction methods . . . . . . 82

4.2 .3 Protein preparation . . . . . . . . . . . . . 83

4.2.4 Ligand preparation and similarity calculation . . . . . . . 84

4.2 .5 Binding mode prediction . . . . . . . . . . . . . 85

4.3 Results and Discussion . . . . . . . . . . . . . . . 87

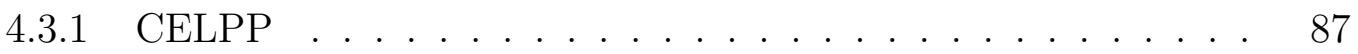

4.4 Conclusion . . . . . . . . . . . . . . . . . . . . . . . 98

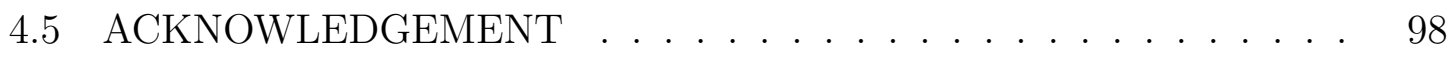

Bibliography . . . . . . . . . . . . . . . . . 99

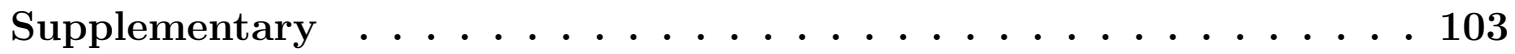

$.1 \quad$ EDockMS Implementation Details Related to Chapter 3. . . . . . . . 103

$.1 .1 \quad$ Server interface $\ldots \ldots \ldots \ldots \ldots$

.1 .2 Database preparation . . . . . . . . . . . . . . . 104

.1 .3 Docking Protocol . . . . . . . . . . . . . . . . . . . . 105

.1 .4 Clustering procedure . . . . . . . . . . . 106

.1 .5 Example and validation of the approach . . . . . . . . 106

Bibliography . . . . . . . . . . . . . . . . . 164

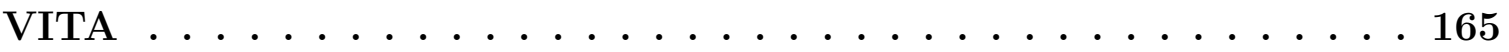




\section{LIST OF TABLES}

Table $\quad$ Page

1.1 Example scoring functions $\ldots \ldots \ldots \ldots$

2.1 The 14 protein kinases for testing the ensemble docking algorithm. The PDB codes of corresponding ligand-bound complexes are listed in the right column with the ligand IDs in parentheses. The protein structure with bold font represents its corresponding protein kinase in ensemble docking calculations. . . . . . . . . . . . .

2.2 A ranked list of 14 protein kinases according to the computed binding energies by ensemble docking experiments for seven typical inhitors. The true targets of each inhibitor are colored in gray. . . . . . . . . 46

2.3 The energy scores of Gleevec binding to 4 homologous tyrosine kinases and to the 14 protein kinases in our ensemble docking test set. The true targets are colored gray. . . . . . . . . . . . . . .

4.1 The results of binding mode prediction for the CELPP targets . . . 88

S1 Table S1 related to Chapter3. EDockMS results of Progesterone. . . . 107

S2 related to Chapter3. Target entries in MDTD . . . . . . . . . . 109 
S3 Table S3, related to Chapter 3. Benchmark for validation . . . . . . 160

S4 Table S4, related to Table 4.1. The results of binding mode prediction for the CELPP targets based on the Vina Score. The first row (Bound) lists the results of docking with the bound protein structures. The last row (hiSHAFTS) gives the results of docking with the user-specified protein structures, and the other five rows show the results of docking using the candidate protein structures provided by CELPP. The error of each value was estimated with the bootstrap method, in which 1000 replicates were used, and was reported in the parentheses. . . . . . . 163 


\section{LIST OF FIGURES}

Figure

Page

1.1 Protein Data Bank growth statistics. ${ }^{14}$ Number of structures deposited per year vs the number of total available structures. . . . . . . . .

1.2 Illustration of ligand-protein docking. Docking algorithms generate a variety of complex configurations. The right panel shows the surface representation of a protein binding pocket, with the ligands in different orientations. . . . . . . . . . . . . . . .

1.3 Illustration of the three conceptual protein-ligand interaction models accompanied with one extended interaction model: (a) The lock-andkey model; both protein and ligand are rigid. (b) The induced-fit model; the conformational change of the protein occurs. (c) The conformational selection model; the ligand binds to the most suitable conformation among an ensemble of protein conformations. (d) The extended conformational selection model; the ligand binds to one protein conformer first and then a subsequent conformational change occurs to the protein. To focus on protein flexibility, this figure does not show ligand flexibility. . . . . . . . . . . . . . . . 
1.4 Stick representation of sugar with the elements in different colors: $\mathrm{C}$ (olive), $\mathrm{O}$ (red), $\mathrm{N}$ (blue). The glycosidic torsion angles $\varphi, \psi$ and $\omega$ are annotated. $\mathrm{C}$ and $\mathrm{O}$ represents carbon and oxygen, respectively. The labeled atoms define the torsion angles as $\varphi\left(\mathrm{O} 5-\mathrm{C} 1-\mathrm{O}_{x}-\mathrm{C}_{x}\right), \psi$ $\left(\mathrm{C} 1-\mathrm{O}_{x}-\mathrm{C}_{x}-\mathrm{C}_{x-1}\right)$ and $\omega(\mathrm{O} 6-\mathrm{C} 6-\mathrm{C} 5-\mathrm{O} 5) \ldots \ldots . \ldots$

1.5 Illustration of dihedral angles in one amino acid residue of a protein: two backbone dihedral angles $\varphi, \psi$ and one side chain dihedral angle $\chi$. The elements are in different colors: $\mathrm{C}($ grey), $\mathrm{O}($ red $)$, and $\mathrm{N}($ blue). Hydrogen atoms are not shown. The the backbone of this amino acid is colored green. . . . . . . . . . . . . . . . . . .

2.1 The success rates in binding mode predictions for 200 complexes at different criteria when the top conformation was considered: rmsd $<$ $1.0,1.5,2.0,2.5$ and $3.0 \AA$, respectively. . . . . . . . . . . . .

2.2 The 2D structures of selected protein kinase inhibitors for ligand selectivity analysis. The ligand IDs with their corresponding PDB codes are shown in the lower-left corner of each cell. This figure is drawn with Marvinsketch, ChemAxon Ltd., http://www.chemaxon.com/.

2.3 (A) The 2D structures of staurosporine. (B) The 2D structures of Gleevec. (C) The binding pocket conformations of PDK1 kinase (sea green) and GSK3 $\beta$ (forest green) bound with staurosporine. (D) Superimposition of the conformation of Gleevec bound to the Tyrosine Kinase domains of Abl(red), c-Kit(orange), Lck(green), and c-Src(blue). Gleevec is shown in the ball \& stick representation. . . . . . . . . . 
2.4 The efficient factors of the ligands in discriminating the true targets from the non-targets predicted by the ensemble docking algorithm. .

2.5 Comparisons of the enrichments between ensemble docking and standard sequential docking on five typical target proteins. The legend applies to all the panels. . . . . . . . . . . . . . . . . . 57

3.1 Schematic representation that highlights the back-end procedures in EDockMS. . . . . . . . . . . . . . . . . .

4.1 The results of binding mode prediction for CELPP using the bound protein structures and the candidate protein structures provided by CELPP for docking. Different RMSD values were used as the thresholds that defined successful predictions. (A) The success rate when only the top predicted model was considered for each protein structure. (B) The success rate when top 5 models were considered for each protein structure. (C) The success rate when all the models were considered for each protein structure. . . . . . . . . . . . . .

4.2 A The results of binding mode prediction for CELPP using the userspecified protein structures (i.e. hiSHAFTS structures in this study) for both docking-based and template-based methods. Different RMSD values were used as the thresholds for the definition of successful predictions. B The correlation between the RMSD values of the binding modes predicted by the template-based method and the corresponding similarity scores between the query ligands and the corresponding template ligands . . . . . . . . . . . . . . . . 
4.3 The top predicted binding mode (colored red) of CatS_1 in comparison with the binding mode given by the crystal structure (colored green) released by D3R. The protein is shown in surface representation. The ligand (Cast_1) is plotted in stick representation. The predicted binding mode was obtained from the docking method using the bound protein structure . . . . . . . . . . . . . . . . . . . . . . . . . 97

S1 Figure S1, related to Chapter3. ROC curves of three benchmark ligands.108

S2 Figure S2, related to Figure 4.1. The results of the binding mode prediction based on the Vina Score. (A) The success rate when only the top predicted model was considered for each protein structure. When $2.0 \AA$ was used as the threshold, the success rates of dockings with the bound, LMCSS, SMCSS, hiResHolo, hiResApo, and hiTanimoto protein structures are $32.8 \%, 22.9 \%, 14.5 \%, 13.8 \%, 7.1 \%, 22.1 \%$, respectively. (B) The success rate when top 5 models were considered for each protein structure. When $2.0 \AA$ was used as the threshold, the success rates of dockings with the bound, LMCSS, SMCSS, hiResHolo, hiResApo, and hiTanimoto protein structures were 44.2\%, 31.3\%, 21.3\%, $21.7 \%, 11.1 \%$, and $30.6 \%$, respectively. . . . . . . . . . . . . 162 


\begin{abstract}
Molecular docking has been a crucial component and remains a highly active area in computer-aided drug design (CADD). In simple terms, molecular docking uses computer algorithms to identify the "best" match between two molecules, a process analogous to solving three-dimensional jigsaw puzzles. In more rigorous terms, the molecular docking problem can be defined as predicting the "correct" bound association state for the given atomic coordinates of two molecules. Docking is an important tool for structure and affinity predictions of molecular association, which would lead to the mechanistic understanding of the physicochemical interactions at the atomic level. Protein-small molecule (referred to as "ligand") docking, in particular, has broad application to structure-based drug design, as drug compounds are usually small molecules. In this dissertation, I present my studies on protein-ligand docking. In the background introduction, I reviewed the docking methodology and the key recent developments in the field. Next, I applied an ensemble docking algorithm onto 14 protein kinases to study ligand selectivity, a major issue for the development of kinase inhibitors as anticancer drugs. In Chapter 3, I developed a web server for automated, in silico screening of multiple targets for a given ligand query. Finally, I integrated the new methods for protein-ligand binding mode prediction and applied the integrated method to a large-scale, blind prediction competition named Continuous Evaluation of Ligand Pose Prediction (CELPP).
\end{abstract}




\section{Chapter 1}

\section{Introduction}

Proteins are incredibly diverse macromolecules made up of amino acids as the building blocks. In a cell, proteins interact with ions, small molecules (drugs), and even macromolecules (e.g., other proteins, nucleic acids). During such interactions, shape alteration (referred to as conformational change) almost occurs at the same time. It is known that a protein structure dictates its function in living organisms. ${ }^{1}$ Any dysfunction could lead to pathological changes. Therefore, studying protein interactions and solving the structures are crucial steps for understanding the mechanism of life, in addition to the investigation of biological systems and rational drug design.

Direct methods for the determination of three-dimensional structures of the proteinligand complexes rely on experiments. Traditional techniques include X-ray crystallography, neutron diffraction or electrons diffraction (which depends on the source type of beams used in interacting with the specimen). ${ }^{2,3}$ The principle of this method is the analysis of diffraction patterns, which requires a crystalline form of the specimen. Proteins are very difficult to crystalize due to the numerous unstructured loops. 
Therefore, the limitation of X-ray crystallography is the quality of the crystallization. In this circumstance, cryo-electron microscopy (cryo-EM) has the advantage that a crystalline sample is not necessary. However, cryo-EM usually has lower resolution when compared with crystallography. Nevertheless, considerable improvements have been made very recently. ${ }^{4-6}$ Also, magnetic resonance (NMR) spectroscopy has contributed to many structural details and dynamics of biological materials. ${ }^{7}$ However, NMR technology has difficulty to solve the structures of large proteins. In summary, all experimental techniques utilized to obtain protein structures have limitations. In addition, they are time consuming and costly. Ultimately, these shortcomings spur the development of computational methods.

Molecular docking is one of the examples that implement the integration of computational and experimental strategies. ${ }^{8-11}$ In the past decades, molecular docking has been a powerful approach for computer-aided drug design (CADD). Generally, molecular docking is performed between two molecules (see Figure 1.2). The target (receptor) is usually a macromolecule such as a protein, DNA or RNA. The other molecule can be a protein, peptide or small molecule. Molecular docking utilizes computational algorithms to predict the bound association of the two molecules. In this work, we focus on docking of small molecules against a protein target, referred to as ligand-protein docking, because of its broad application to structure-based drug design (SBDD) (drug compounds are usually small molecules). With a protein of a known three-dimensional structure, the docking method can predict how ligands interact with their protein targets from a physical or chemical perspective. The determination of protein-ligand complex structures can be done routinely by docking algorithms. In other words, protein-ligand docking approaches allow an automatic 


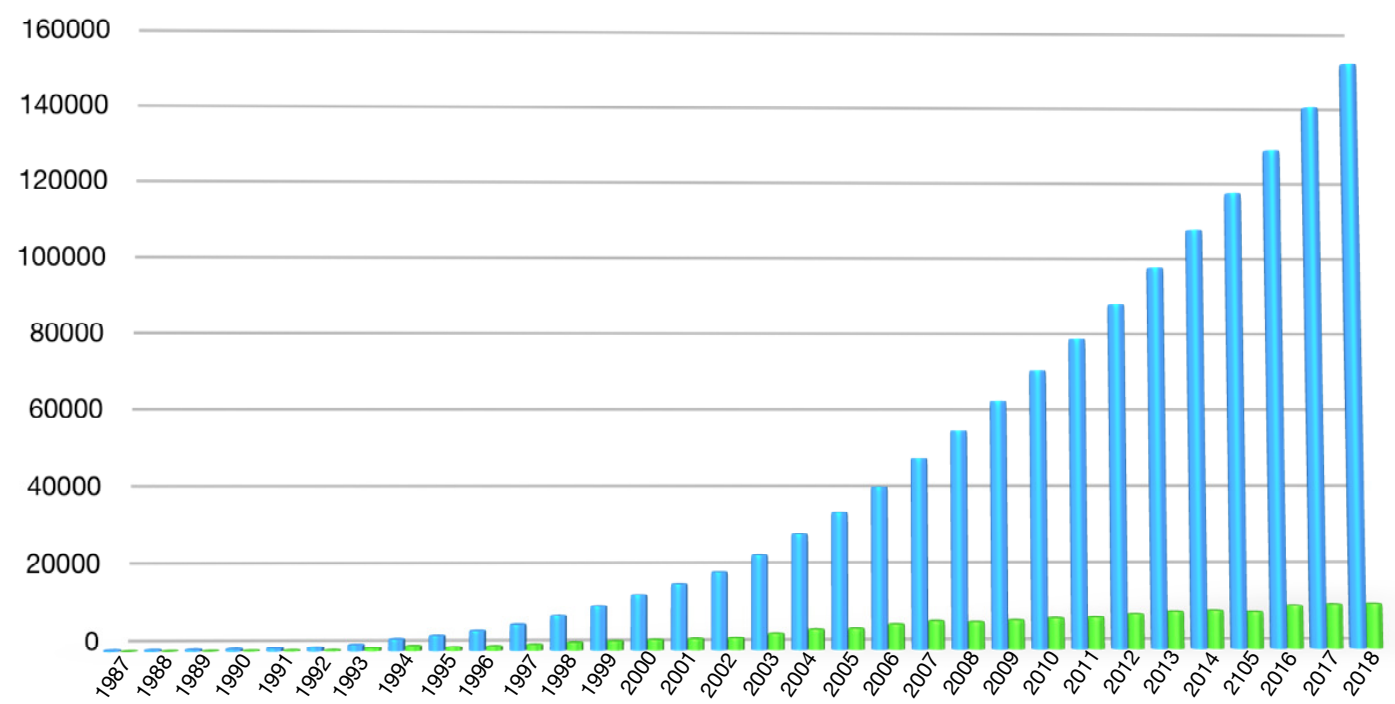

Figure 1.1: Protein Data Bank growth statistics. ${ }^{14}$ Number of structures deposited per year vs the number of total available structures.

way to manipulate the recognition of a drug by its protein target through capturing physical principles.

Nowadays, protein-ligand docking is widely applied to structure-activity and mutagenesis studies, virtual screening (VS), and lead optimization. ${ }^{12}$ With the rapid growth of the protein structures in the Protein Data Bank $\left.{ }^{13,14}\right)$, protein-ligand docking methods have become a valuable tool for mechanistic biological research and pharmaceutical drug discovery. 


\subsection{Physical Basis of Molecular Docking}

\subsubsection{Protein-ligand interaction}

Protein-ligand interactions are central to the in-depth understanding of protein functions in biology because proteins accomplish molecular recognition through binding with various molecules. ${ }^{15,16}$ Drugs often act as inhibitors when interacting with proteins, because many diseases including cancer are attributed to the abnormally active protein-ligand interactions. In this circumstance, inhibitors can be used to prevent the abnormal interactions for specific therapy. For instance, in the treatment of chronic myelogenous leukemia (CML), STI571 (Gleevec ${ }^{\mathrm{TM}}$, imatinib mesylate) is used to inhibit Bcr-Abl target protein activation, which also exemplifies the successful development of the rational drug design. ${ }^{17}$ Therefore, insights into protein-ligand interactions are vital for the development of drugs.

Molecular docking algorithms predict protein-ligand interactions in a complex that is formed non-covalently. In biological systems, hydrogen bonds, ionic bonds, Van der Waals interactions and hydrophobic bonds are the four main types of non-covalent interactions. ${ }^{18}$ Non-covalent bonds, ranging from $1-5 \mathrm{kcal} / \mathrm{mol},{ }^{19}$ are weak in comparison with covalent bonds. Therefore, most of the time the term "non-covalent interactions" is used, rather than non-covalent bonds. However, the cumulative effect of these non-covalent interactions can be significant, which means multiple non-covalent bonds often act together at the binding interface of a complex to produce highly stable and specific associations. ${ }^{20}$ 


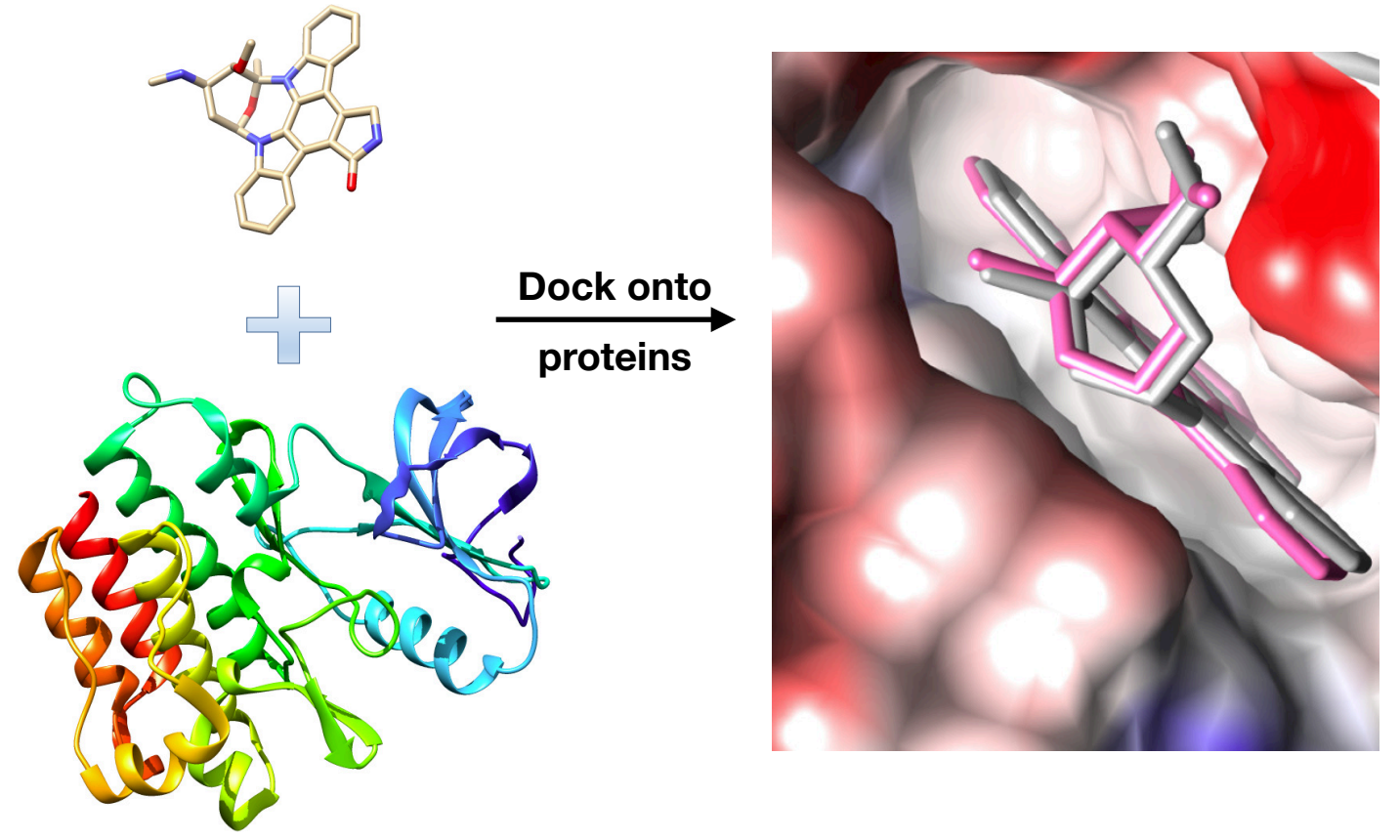

Figure 1.2: Illustration of ligand-protein docking. Docking algorithms generate a variety of complex configurations. The right panel shows the surface representation of a protein binding pocket, with the ligands in different orientations. 


\section{Hydrogen bonds}

Hydrogen bonds are polar electrostatic interactions that can be described in the form of $\mathrm{D}-\mathrm{H} \cdots \mathrm{A}$. Here, $\mathrm{D}$ and $\mathrm{A}$ stand for an electron donor and acceptor atom, respectively. $\mathrm{H}$ is the hydrogen atom attached to a donor atom. Thus, the donor atom must be electronegative. ${ }^{21}$ Hydrogen bonds have a strength of about $5 \mathrm{kcal} / \mathrm{mol}$, which is weaker than the covalent bond between an oxygen atom and a hydrogen atom (approximately $110 \mathrm{kcal} / \mathrm{mol}) \cdot{ }^{19}$ However, biomolecules exist in solvent surroundings, in which the extensive hydrogen bonds between adjacent molecules are broken and reformed constantly, resulting in the change of system enthalpy and entropy. Both the protein and the ligand interact with the solvent before binding, so the enthalpy

and entropy influence their complex formation. ${ }^{8,15}$ Also, hydrogen bonds contribute a stabilizing force to macromolecules (e.g., proteins and nucleic acids), which is of great importance to the structure.

\section{Ionic bonds}

Ionic interaction is the electronic attraction between oppositely charged ionic pairs. Thus, ionic bonds are highly specific electrostatic interactions. In the aqueous solution, ions are surrounded by a shell of water molecules which is caused by dissolution in water. ${ }^{20,22,23}$

\section{Van der Waals interactions}

When atoms in different molecule are close enough, the transient dipoles in the electron clouds lead to an intermolecular force and form nonspecific Van der Waals interactions. Roughly, the strength of Van der Waals interaction is $1 \mathrm{kcal} / \mathrm{mol}$, which 
is even weaker than typical hydrogen bonds.

\section{Hydrophobic interactions}

Hydrophobic interactions result from the effect that nonpolar molecules tend to exclude from the solvent and aggregate in an adequate surrounding. Hydrophobic inter-

actions are often considered as driven by the entropy gain. ${ }^{23}$ However, the molecular mechanisms of the hydrophobic effect remain controversial. In the scaled-particle theory, the hydrophobic effect is multifaceted depending on the size of solute. ${ }^{23,24}$

\subsubsection{Enthalpy-Entropy Compensation}

Besides the aforementioned major types of non-covalent interactions, other types of non-covalent interactions also exist in protein-ligand complexes, such as $\mathrm{CH}-\pi$ in protein-sugar complexes due to the presence of aromatic rings. ${ }^{25}$ To be noted, the solvent is always considered in the system when we discuss about non-covalent interactions between the protein and the ligand. In fact, numerous solvent molecules are involved in binding and the solvent effect plays an important role in protein-ligand interactions. The contributions of various non-covalent interactions are closely related to two thermodynamic quantities, entropy and enthalpy. The enthalpic and entropic changes before and after the complex formation determine how tightly the protein and ligand bind together, which is characterized by the Gibbs binding free energy, as shown in Equation 1.1.

$$
\Delta G_{\text {bind }}=\Delta H-T \Delta S
$$


Here, $\Delta G$ represents the change of the free energy, and $\mathrm{T}$ is the absolute temperature in Kelvin. $\Delta H$ represents the types and numbers of chemical bonds and noncovalent interactions that are broken and formed, and $\Delta S$ is the change in the randomness of the system. The Gibbs binding free energy can be calculated using theoretical methods, or can be quantified experimentally by the reaction rate: ${ }^{26}$

$$
\Delta G_{b i n d}=-R T \ln K_{e q}=-R T \ln \frac{k_{o n}}{k_{o f f}}
$$

Equation 1.2 shows that complex stability can be measured through the equilibrium binding constant $\mathrm{K}_{e q}$, which is determined by the two kinetic rate constants, $\mathrm{K}_{o n}$ (for the binding reaction) and $\mathrm{K}_{\text {off }}$ (for the dissociation reaction). $\mathrm{R}$ and $\mathrm{T}$ is the gas constant (8.314 J/mol.K.) and the absolute temperature, respectively.

The net driving force for binding is balanced between the two factors, entropy (the tendency to achieve the highest degree of randomness) and enthalpy (the tendency to achieve the most stable bonding state). In fact, the protein-ligand binding process is driven by the decrease in the total Gibbs free energy of the system. ${ }^{15}$

\subsubsection{Molecular recognition models}

Noncovalent interaction-driven molecular complexes show geometrical complementarity. Three conceptual models of ligand-protein binding have been proposed to explain the mechanism for molecular recognition (Figure 1.3). Fisher's lock-and-key model theorizes that the binding interface should be matched complementarily. ${ }^{27}$ The protein and the ligand are rigid in this model, which means that their conformations are 
identical before and after binding. Du et al. suggested that the lock-and-key model is an entropy-dominated binding process. ${ }^{15}$

In Koshland's induced-fit model, conformational change occurs to the protein during binding in order to accommodate the ligand with the best amino acid configuration. ${ }^{28}$ It should be noted that in most cases only minor conformational changes arise in proteins after ligand binding. Compared with the lock-and-key model, the induced-fit hypothesis can be linked to a "hand in glove" model, adding flexibility upon Fisher's idea. ${ }^{29}$

In the conformational selection model, ligands bind selectively to the most suitable conformational state among an ensemble substates. ${ }^{30,31}$ In the original conformational selection model, no further conformational rearrangement occurs. However, in an extended conformational selection recognition mechanism, ligands first bind to a protein in the initial favorable protein conformational state, which induces a protein conformational change subsequently. In other words, the "induced-fit" and "conformational selection" recognition mechanisms may co-exist in ligand binding. ${ }^{32,33}$ 
(a)

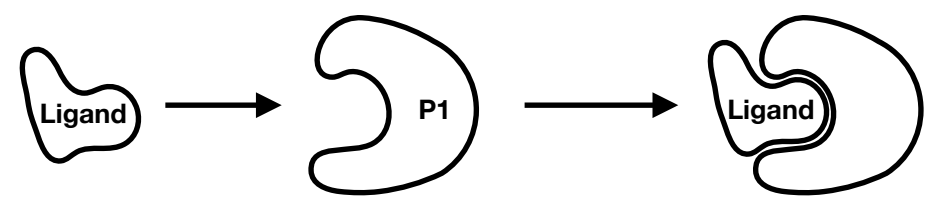

(b)

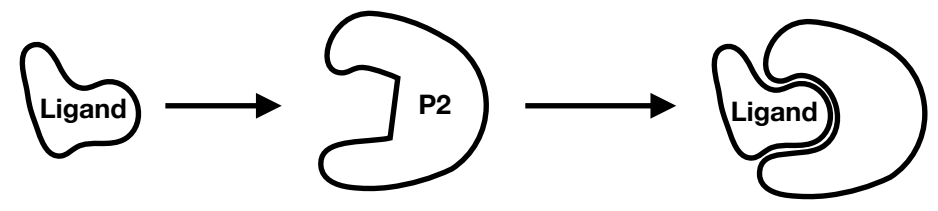

(c)

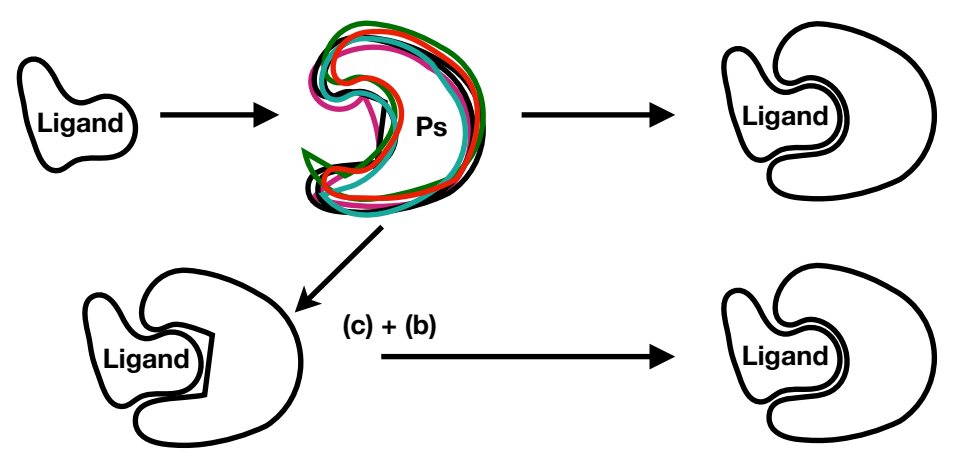

Figure 1.3: Illustration of the three conceptual protein-ligand interaction models accompanied with one extended interaction model: (a) The lock-and-key model; both protein and ligand are rigid. (b) The induced-fit model; the conformational change of the protein occurs. (c) The conformational selection model; the ligand binds to the most suitable conformation among an ensemble of protein conformations. (d) The extended conformational selection model; the ligand binds to one protein conformer first and then a subsequent conformational change occurs to the protein. To focus on protein flexibility, this figure does not show ligand flexibility. 


\section{$1.2 \quad$ Key Components in Ligand-Protein Docking}

The basic procedure of docking can be simplified to sampling and ranking, two critical ingredients in current docking programs. Sampling refers to searching putative ligand conformations in the binding site of a receptor. Proteins are usually held rigid in most docking algorithms. Generation of 3D conformers for the ligand is required for flexible (ligand) docking. A good sampling algorith should be able to generate a diverse ensemble of ligand structures, including the bioactive, bound ligand structure. The second component, ranking, refers to assessing the fit between a ligand conformation and the target by scoring functions. These two docking components will be discussed in detail, respectively.

\subsubsection{Conformational sampling}

Biomolecules are dynamic; they are either dissolved in the cytosol or associated with some other components of the cell. Besides the six translational and rotational degrees of freedom, dihedral (torsional) angles also add up extensive flexibility. For example, in sugar molecules, the diversity stems primarily from the rotatable bonds that connect monosaccharides, which are also known as glycosidic linkages (see Figure 1.4). Sugars are intricate biomolecules, as being indicated by their nomenclature, reactions, functions, and linear/branched structures. More diverse conformational sampling is required with a larger number of monosaccharides in an oligosaccharide chain. ${ }^{34-36}$

Similarly, proteins are polymers of amino acids that link together through peptide bonds. Therefore, even small variations in the conformations of protein subunits 


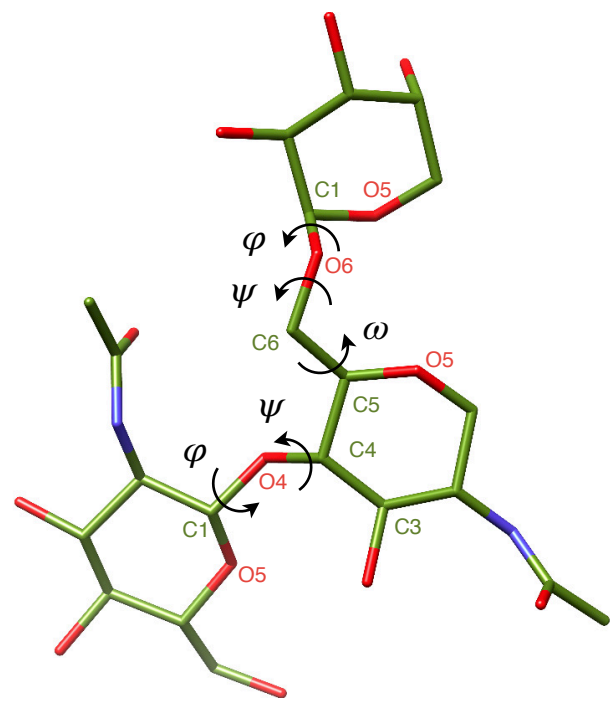

Figure 1.4: Stick representation of sugar with the elements in different colors: $\mathrm{C}$ (olive), $\mathrm{O}$ (red), $\mathrm{N}$ (blue). The glycosidic torsion angles $\varphi, \psi$ and $\omega$ are annotated. $\mathrm{C}$ and $\mathrm{O}$ represents carbon and oxygen, respectively. The labeled atoms define the torsion angles as $\varphi\left(\mathrm{O} 5-\mathrm{C} 1-\mathrm{O}_{x}-\mathrm{C}_{x}\right), \psi\left(\mathrm{C} 1-\mathrm{O}_{x}-\mathrm{C}_{x}-\mathrm{C}_{x-1}\right)$ and $\omega(\mathrm{O} 6-\mathrm{C} 6-\mathrm{C} 5-\mathrm{O} 5)$.

could lead to extensive flexibility in a protein. Figure 1.5 shows the dihedral angles of an amino acid in a protein. The third backbone dihedral angle $\omega$ (see Figure 1.4 for its definition) is typically close to $180^{\circ}$ or $0^{\circ}$ in proteins. The flexible side chain dihedral angle for each residue has various distributions. ${ }^{37}$ Furthermore, the task is more complicated because proteins in solution exist as an ensemble of conformations and flucuate over time, which make it even harder to simulate on computer. While more extensive samplings are needed to account for the induced fit and conformational selection models as we introduce in section 1.1.3. Our group has developed a docking package to consider many different conformations in an ensemble without time consuming strain. We will introduce it in section 1.2.3 and section 1.3. 


\subsubsection{Sampling algorithms}

Computational conformational sampling has been an active realm for many years because of evolving small molecule modeling and design in pharmaceutical work. ${ }^{38,39}$ Sampling algorithms explore the molecules conformational space from two perspectives. One of the categories exhaustively enumerates all possible torsions, which is necessary due to our limited knowledge of pharmaceutically relevant conformational space. However, this method may cause exponential growth of the search space. To overcome high degrees of freedom, systematic search methods store the physicochemical properties in an applicate grid to prevent combinatorial explosion. ${ }^{40}$ The typical procedures divide the ligand into fragments first and then dock into the active site sequentially. An application of automated calculating grids is incremental construction (IC) methods (anchor and grow), which are implemented in the application DOCK. ${ }^{41}$ DOCK is the first and most widely used docking program, and the newest version is DOCK 6. ${ }^{42,43}$ Other well known flexible ligand searching programs such as FlexX and Glide also use incremental construction methods. ${ }^{44,45}$

The other perspective uses a random element to change the system degrees of freedom, which is also referred to as stochastic search. Most common stochastic methods include genetic algorithms (GAs) and Monte Carlo (MC) search. ${ }^{46,47}$ Also, simulation methods have been developed and widely used in molecular docking sampling procedures. Molecular dynamics (MD) is one example of simulation methods that the generated state has to be lower in energy than the initial state to move to the next step. Therefore, it may often get trapped in the local minima that cannot cross high energy barriers. An alternative strategy is to identify the conformation preliminarily, and followed by further MD simulations to implement local optimiza- 


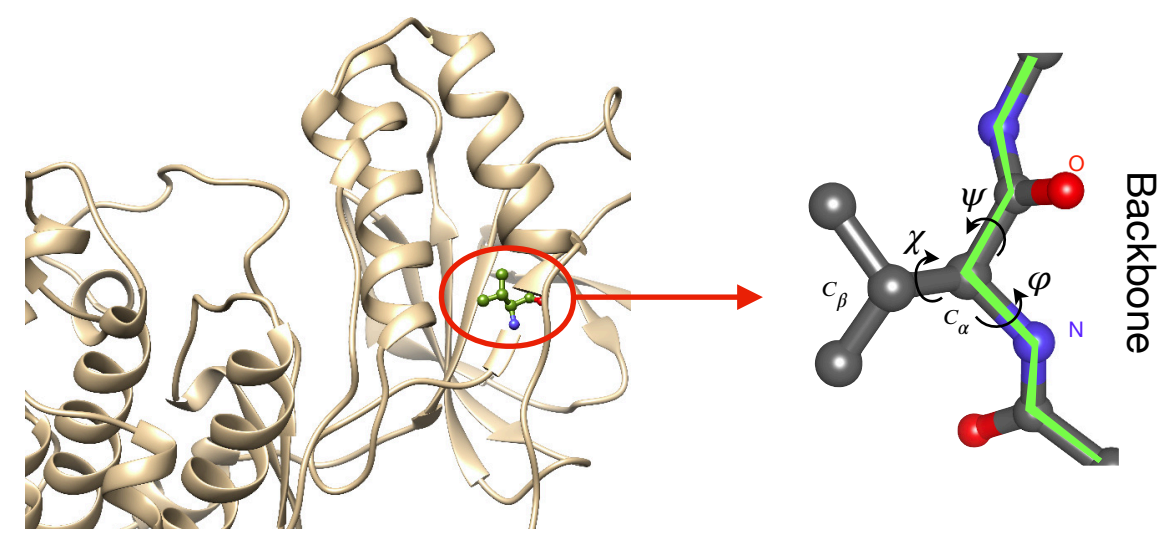

Figure 1.5: Illustration of dihedral angles in one amino acid residue of a protein: two backbone dihedral angles $\varphi, \psi$ and one side chain dihedral angle $\chi$. The elements are in different colors: $\mathrm{C}$ (grey), $\mathrm{O}$ (red), and $\mathrm{N}$ (blue). Hydrogen atoms are not shown. The the backbone of this amino acid is colored green.

tion. Other methods such as increasing the simulation temperature and smoothing potential energy surfaces are also introduced in previous studies. ${ }^{8,39}$

\subsubsection{Scoring functions}

Another key aspect of molecular docking is to rank the ligand conformations that have been generated. A good scoring function should be capable of distinguishing between the true binding modes and the decoy modes. Due to the large degrees of freedom in the protein-ligand system, balancing the speed and accuracy is necessary for the scoring step. The scoring functions are mainly divided into three categories: force field-based, empirical, and knowledge-based potentials. Different scoring functions are listed in Table 1.1. 


\section{Force field-based scoring functions}

Classical force field-based scoring functions calculate Coulombic (for electrostatics) and Lennard-Jones (for Van der Waals) terms for non-covalent interactions. More sophisticated forms as an extension of force field-based scoring functions also consider the terms of hydrogen bonds, solvations and entropic contributions.

\section{Empirical scoring functions}

Empirical scoring functions are often related to force field-based scoring functions because they consider similar molecular contributions such as hydrogen bonds, ionic interactions, hydrophobic effects and entropic change. Summation of all these components results in the binding free energy, which is ultimately calculated by empirical scoring functions. The empirical coefficients are trained by fitting the scoring function to the experimentally measured binding affinities. Therefore, these coefficients strongly rely on the experimental data set for fitting, which is the main drawback of empirical scoring functions.

\section{Knowledge-based scoring functions}

Knowledge-based scoring functions derive atomic pair potentials by statistical principles to extract preferred interaction geometries. ${ }^{48}$ High efficiency is one of the major characteristics of knowledge-based scoring functions, in addition to the implicit consideration of the solvent effect, resulting in more common application of knowledgebased scoring functions than other scoring functions. ${ }^{49,50}$ Example knowledge-based scoring functions include DrugScore, ${ }^{49}$ PMFScore, ${ }^{51}$ and ITScore. ${ }^{52,53}$ 


\section{Recent development in scoring functions}

It is worth noting that there is no universal scoring function that can fit all biological systems, and it is not appropriate to do so because the existing scoring functions remain to have limitations and most of them are sensitive to the training set or have a limited scope of application. Therefore, when docking against a specific target family, training a scoring function with the particular protein family can usually improve binding mode and binding affinity prediction, which is referred to as target-specific scoring functions. ${ }^{54}$ The consensus scoring approach is another way to overcome the deficiencies of individual scoring functions to some extent. Consensus scoring functions can mitigate the bias of a single scoring function and improve the hit rate. ${ }^{55,56}$ The consensus appoach should not include multiple scoring functions that yield highly correlated results; otherwise the bias of these scoring functions would be amplified rather than diminished. ${ }^{55}$ Other ongoing methodology development includes adding additional terms to the existing scoring functions to improve the scoring performance. ${ }^{57,58}$ Numerous comparison studies have been reported to evaluate the performance of different scoring functions. ${ }^{50,59-62}$

\subsection{Docking Programs}

In the past two decades, more than 60 docking programs have become available, ${ }^{63}$ such as SLIDE, ${ }^{64}$ X-CSCORE ${ }^{65}$ and ConsDock. ${ }^{66}$ Most of the existing docking algorithms treat ligands as flexible molecules and proteins as rigid molecules, because a protein

usually consists of thousands of atoms. In some programs such as GalaxyDock, ${ }^{67}$ protein side-chains can be optimized. 
Moreover, there are indirect ways to consider the protein induced fit effect in ligand binding. A common approach is to utilize ensemble protein conformations. These ensemble protein structures can be obtained either from computational analysis such as molecular dynamics (MD) simulations or from experimental measurements such as NMR or X-ray diffraction. The MDock software incorporates a method referred to as "ensemble docking" to implicitly account for protein flexibility. In this method, the protein conformational number is introduced as an additional variable during docking optimization. This ensemble docking algorithm is computationally efficient; its computational speed is comparable to that of single-protein docking. ${ }^{52,53,68}$ Mizutani et al. used an enlarged binding pocket in their docking program ADAM to allow for certain protein flexibility. ${ }^{69}$ In this approach, the van der Waals energy curve for each atom pair is shiftied to widen the protein cavity uniformly, followed by structure optimization to remove atomic clashes and to improve interaction energy. Similarly, Bottegoni et al. adopted the SCARE (SCan Alanines and REfine) protocol, which enlarges the binding pocket by alanine mutation within the binding pocket. ${ }^{70}$

In order to improve the docking success, some docking programs target specific protein or ligand classes. Rather than generating a "universal scoring function", training a system-dependent scoring function or adding additional items in energy calculations can be beneficial to the prediction accuracy. For example, Vina-carb ${ }^{71}$ integrated the Carbohydrate Intrinsic (CHI) energy terms into the AutoDock Vina scoring function (ADV), improving the docking accuracy from $55 \%$ to $74 \%$ compared with the use of the ADV score alone. 
Table 1.1: Example scoring functions

\begin{tabular}{|c|c|c|c|}
\hline Scoring function & Category & Prominent features & Ref \\
\hline GOLDScore & force field-based & Default scoring function for GOLD & 47 \\
\hline MedusaScore $^{b}$ & force field-based & $\begin{array}{l}\text { Including a hydrogen-bonding model and im- } \\
\text { plicit solvent model }\end{array}$ & 72 \\
\hline DOCK6 & $\begin{array}{l}\text { force field-based and } \\
\text { footprint similarity }\end{array}$ & Updated internal energy function & 43 \\
\hline Glide & $\begin{array}{l}\text { force field-based and } \\
\text { empirical }\end{array}$ & $\begin{array}{l}\text { Modifying and expanding the ChemScore } \\
\text { function }\end{array}$ & 45 \\
\hline AutoDock $^{a}$ & empirical & Allowing side chains to be flexible & 73 \\
\hline AutoDockFR ${ }^{a}$ & empirical & Considering partial receptor flexibility & 74 \\
\hline AutoDock VinaXB & $\begin{array}{l}\text { halogen bond scoring } \\
\text { function }\end{array}$ & Adding halogen bonding parameters & 75 \\
\hline $\mathrm{ICM}$ & empirical & $\begin{array}{l}\text { Allowing flexible side chains which are sam- } \\
\text { pled simultaneously with the ligand }\end{array}$ & 76 \\
\hline LigScore1/LigScore2 & empirical & $\begin{array}{l}\text { Consisting of three terms that describe the van } \\
\text { der Waals interaction }\end{array}$ & 77 \\
\hline Vina & empirical & $\begin{array}{l}\text { Summation of the energetic contributions } \\
\text { from both inter- and intra-molecular interac- } \\
\text { tions }\end{array}$ & 78 \\
\hline Vinardo & empirical & $\begin{array}{l}\text { Modifying the interaction terms and the } \\
\text { atomic radii of the Vina scoring function }\end{array}$ & 58 \\
\hline Vina-Carb & empirical & $\begin{array}{l}\text { Adding a CHI-energy term to the scoring func- } \\
\text { tion of AutoDock Vina to improve protein- } \\
\text { carbohydrate docking }\end{array}$ & 71 \\
\hline SLICK & empirical & $\begin{array}{l}\text { A scoring function for protein-carbohydrate } \\
\text { docking by implementing a } \mathrm{CH}-\pi \text { stacking in- } \\
\text { teraction energy term in BALLDock }\end{array}$ & 79 \\
\hline DrugScore & knowledge-based & $\begin{array}{l}\text { Extracting both short-range pair and solvent } \\
\text { accessible surface potentials }\end{array}$ & 49 \\
\hline PFM & knowledge-based & $\begin{array}{l}\text { Potentials of mean force for fast calculation of } \\
\text { atom pair interaction potentials }\end{array}$ & 51 \\
\hline ITScore & knowledge-based & $\begin{array}{l}\text { An iterative method to derive atomic pair po- } \\
\text { tential energies }\end{array}$ & 52 \\
\hline ROTA & knowledge-based & pair potentials in GalaxyDock ${ }^{a}$ & 67,80 \\
\hline AffiScore/N & knowledge-based & $\begin{array}{l}\text { A scoring function in SLIDE for the secondary } \\
\text { scoring step }\end{array}$ & 64 \\
\hline X-CSCORE & consensus & Combine three empirical scoring functions & 65 \\
\hline
\end{tabular}




\subsection{Structure-based virtual screening and inverse virtual screening}

The applications of molecular docking in therapy interventions can be bidirectional depending on the purpose of docking. Conventional docking searches for a compound from a drug bank or from a compound library, a process referred to as virtual screening (VS). ${ }^{55,81}$ On the other hand, for a specific ligand of interest, inverse docking is useful for identification of the targets from thousands of protein candidates, a process called inverse virtual screening (IVS). ${ }^{56,82-84}$ Both prediction tasks are challenging and require immense calculations.

Virtual screening methods are classified into structure-based virtual screening and ligand-based virtual screening. ${ }^{85}$ Structure-based virtual screening is explored by docking methods on a large scale. In contrast, ligand-based virtual screening methods utilize all the structural and chemical information (e.g., by searching the common features or similarity) rather than using a docking strategy. These two methods are not competitive, and can be used individually or in combination. For instance, a ligand-based prescreening step can be done for preselection in order to reduce the size of the compound database in many structure-based virtual screening applications. ${ }^{55}$ Tan et al. integrated the two methods into parallel selection and postulated that structure-based and ligand-based screening could be more effective in a complementarity manner than rank fusion. ${ }^{86}$

IVS reverse docking has been widely applied to drug development in recent years. ${ }^{82,87}$ Screening from a drug target database can help rapidly identify potential target candidates for a specific molecule. The molecule can be a non-drug ligand or a known drug. The major safety issues of drug candidates are possible side effects (referred 
to as off-target effects) and toxicity. IVS can help investigate the interactions of the drug candidate with multiple targets in early-stage drug development. The purpose of IVS studies on known drugs is to search for alternative uses of FDA approved drugs, which reduce costs and risks in comparison with new chemical compounds. This approach is referred to as drug-repositioning (also known as drug repurposing or reprofiling). ${ }^{88-90}$ Drug development has been trending from "one gene, one drug, one disease" to polypharmacology, which means that drugs are designed to interact with multiple targets. The main challenge of IVS is the costly computational time. Recently, we have integrated our fast docking application, MDock, into a webserver platform in order to undertake the IVS tasks. The server is built-in with 3268 proteinligand complexes (containing 3349 distinct binding sites) associated with 537 protein targets. A typical IVS run for a given ligand takes 1 to 4 hours. For the example, the IVS study on the progesterone receptor takes approxiamately one hour on a workstation with 24 Intel Xeon cores [Intel(R) Xeon(R) CPU E5-2650 v3 @ 2.30GHz]. In addition, the screening results from our web server provide searchable target diseases information. ${ }^{91}$

\subsection{Challenges}

Molecular docking methodology is rapidly evolving and improving with the exponential increase of computing power. Recently, machine learning algorithms (including neural network and deep learning) $)^{92,93}$ and hybrid approaches from high-content screening and virtual screening ${ }^{94}$ have been proposed for docking predictions and drug discovery. A critical question in docking is how reliable the docking predic- 
tions are Chen. ${ }^{95}$ Consequently, benchmarking excises for blind docking prediction have been held, such as Community Structure-Activity Resource (CSAR), ${ }^{96}$ Drug Design Data Resource (D3R) ${ }^{97-99}$ and Continuous Evaluation of Ligand Pose Prediction (CELPP). ${ }^{100}$ Our group participate in the weekly competition (CELPP) using a fully automated systematic strategy. ${ }^{101}$ These large-scale protein-ligand complex structure predictions are statistically significant. Through our analysis, the current sampling (80.4\%) is much better than the scoring (46.3\%). ${ }^{101}$ The inaccuracy of current scoring functions likely arises from various sources, such as solvent effects, entropic effects, protonation or tautomer states, and so on. Development of new scoring schemes to address these issues is at large. The biggest hurdle in molecular docking stems from protein flexibility. The computational cost is immense for sampling all possible protein conformations. In conclusion, despite decades of docking algorithm development, prediction accuracy remains a major issue.

This dissertation presents my study on protein-ligand binding mode predictions and inverse virtual screening. In Chapter 2, I will present my study of virtual screening against 14 human protein kinases for ligand selectivity. Protein kinases have large population in the human body; up to $30 \%$ of all human proteins may be modified by kinase activity. Ligand selectivity is a big hurdle for the development of kinase inhibitors as anticancer drugs. We have presented an efficient ensemble docking algorithm to simultaneously address multiple target proteins. The algorithm was validated by virtual screening against 14 human protein kinases. The ensemble docking algorithm performed comparably to standard single-target docking without a significant increase in run time. We expect the algorithm to be useful for the investigation of the ligand selectivity in structure-based drug design. 
In Chapter 3, I developed a ligand-protein docking server to predict potentially promising therapeutic targets for a query compound of interest. I also constructed a protein target database, which contains 537 targets, 3268 protein-ligand complex structures, and 3349 binding pockets. Our achieved success rates and computational efficiency suggest the server to be an effective tool for therapeutic intervention.

Chapter 4 describes the new methods we developed for protein-ligand binding mode prediction during the participation in the D3R Challenges. These methods were integrated, automated, and systematically tested using the large-scale data from Continuous Evaluation of Ligand Pose Prediction (CELPP) and a subset of Grand challenge 3 (GC3). The binding mode prediction results were thoroughly analyzed. 


\section{Bibliography}

[1] Starr, C.; Taggart, R.; Evers, C.; Starr, L. Biology: The Unity and Diversity of Life; Cengage Learning, 2015.

[2] Rossmann, M. G.; Morais, M. C.; Leiman, P. G.; Zhang, W. Structure 2005, 13, $355-362$.

[3] Gallagher-Jones, M.; Rodriguez, J. A.; Miao, J. Quarterly Reviews of Biophysics 2016, 49 .

[4] Callaway, E. Nature News 2015, 525, 172.

[5] Glaeser, R. M. Nature methods 2015, 13, 28.

[6] Vinothkumar, K. R.; Henderson, R. Quarterly reviews of biophysics 2016, 49.

[7] Price, W. S. Concepts in Magnetic Resonance Part A 2009, 34, 60-61.

[8] Brooijmans, N.; Kuntz, I. D. Annual review of biophysics and biomolecular structure 2003, 32, 335-373.

[9] Meng, X.-Y.; Zhang, H.-X.; Mezei, M.; Cui, M. Current computer-aided drug design 2011, 7, 146-157. 
[10] Grinter, S. Z.; Zou, X. Molecules 2014, 19, 10150-10176.

[11] Ferreira, L. G.; dos Santos, R. N.; Oliva, G.; Andricopulo, A. D. Molecules 2015, 20, 13384-13421.

[12] Morris, G. M.; Lim-Wilby, M. Molecular modeling of proteins; Springer, 2008; pp 365-382.

[13] Overington, J. P.; Al-Lazikani, B.; Hopkins, A. L. Nature reviews Drug discovery 2006, 5, 993.

[14] Bernstein, F. C.; Koetzle, T. F.; Williams, G. J.; Meyer, E. F.; Brice, M. D.; Rodgers, J. R.; Kennard, O.; Shimanouchi, T.; Tasumi, M. Journal of Molecular Biology 1977, 112, 535-542.

[15] Du, X.; Li, Y.; Xia, Y.-L.; Ai, S.-M.; Liang, J.; Sang, P.; Ji, X.-L.; Liu, S.-Q. International journal of molecular sciences 2016, 17, 144.

[16] Chandel, T. I.; Zaman, M.; Khan, M. V.; Ali, M.; Rabbani, G.; Ishtikhar, M.; Khan, R. H. International Journal of Biological Macromolecules 2018, 106, 11151129.

[17] Druker, B. J. Trends in molecular medicine 2002, 8, S14-S18.

[18] K., A. Thermodynamics - Interaction Studies - Solids, Liquids and Gases; InTech, 2011.

[19] others,, et al. Molecular cell biology; Macmillan, 2008.

[20] Nelson, D. L.; Cox, M. M. Lehninger Principles of Biochemistry, Fourth Edition; W. H. Freeman, 2004. 
[21] Baker, E. N. International Tables for Crystallography; International Union of Crystallography, 2006; pp 546-552.

[22] Ninham, B. W.; Yaminsky, V. Langmuir 1997, 13, 2097-2108.

[23] Chandler, D. Nature 2005, 43\%, 640.

[24] Snyder, P. W.; Mecinović, J.; Moustakas, D. T.; Thomas, S. W.; Harder, M.; Mack, E. T.; Lockett, M. R.; Héroux, A.; Sherman, W.; Whitesides, G. M. Proceedings of the National Academy of Sciences 2011,

[25] Muraki, M.; Ishimura, M.; Harata, K. Biochimica et Biophysica Acta (BBA) General Subjects 2002, 1569, 10-20.

[26] Olsson, T. S.; Williams, M. A.; Pitt, W. R.; Ladbury, J. E. Journal of Molecular Biology 2008, 384, 1002-1017.

[27] Fischer, E. Ber. Dtsch. Chem. Ges 1894, 27, 2985-2993.

[28] Koshland, D. Proceedings of the National Academy of Sciences 1958, 44, 98-104.

[29] Koshland Jr, D. Angewandte Chemie-English Edition 1994, 33, 2475.

[30] Ma, B.; Kumar, S.; Tsai, C.-J.; Nussinov, R. Protein engineering 1999, 12, 713-720.

[31] Tsai, C.-J.; Kumar, S.; Ma, B.; Nussinov, R. Protein Science 1999, 8, 1181-1190.

[32] Boehr, D. D.; Nussinov, R.; Wright, P. E. Nature chemical biology 2009, 5, 789.

[33] Csermely, P.; Palotai, R.; Nussinov, R. Trends in biochemical sciences 2010, 35, 539-546. 
[34] DeMarco, M. L.; Woods, R. J. Glycobiology 2008, 18, 426-440.

[35] Agirre, J. Acta Crystallographica Section D 2017, 73, 171-186.

[36] Lütteke, T. Acta Crystallographica Section D: Biological Crystallography 2009, $65,156-168$.

[37] Zhou, A. Q.; O'Hern, C. S.; Regan, L. Proteins: Structure, Function, and Bioinformatics 2014, 82, 2574-2584.

[38] Leach, A. R. Reviews in Computational Chemistry; John Wiley \& Sons, Inc., 2007; pp 1-55.

[39] Agrafiotis, D. K.; Gibbs, A. C.; Zhu, F.; Izrailev, S.; Martin, E. Journal of chemical information and modeling 2007, 47, 1067-1086.

[40] Goodford, P. J. Journal of medicinal chemistry 1985, 28, 849-857.

[41] Meng, E. C.; Shoichet, B. K.; Kuntz, I. D. Journal of computational chemistry $1992,13,505-524$.

[42] Kuntz, I. D.; Blaney, J. M.; Oatley, S. J.; Langridge, R.; Ferrin, T. E. Journal of Molecular Biology 1982, 161, 269-288.

[43] Allen, W. J.; Balius, T. E.; Mukherjee, S.; Brozell, S. R.; Moustakas, D. T.; Lang, P. T.; Case, D. A.; Kuntz, I. D.; Rizzo, R. C. Journal of Computational Chemistry 2015, 36, 1132-1156.

[44] Rarey, M.; Kramer, B.; Lengauer, T.; Klebe, G. Journal of molecular biology 1996, 261, 470-489. 
[45] Friesner, R. A.; Banks, J. L.; Murphy, R. B.; Halgren, T. A.; Klicic, J. J.; Mainz, D. T.; Repasky, M. P.; Knoll, E. H.; Shelley, M.; Perry, J. K.; Shaw, D. E.; Francis, P.; Shenkin, P. S. Journal of Medicinal Chemistry 2004, 47, 1739-1749.

[46] Bean, J. C. ORSA journal on computing 1994, 6, 154-160.

[47] Jones, G.; Willett, P.; Glen, R. C.; Leach, A. R.; Taylor, R. Journal of Molecular Biology 1997, 267, 727-748.

[48] Gohlke, H.; Klebe, G. Current opinion in structural biology 2001, 11, 231-235.

[49] Gohlke, H.; Hendlich, M.; Klebe, G. Journal of molecular biology 2000, 295, $337-356$.

[50] Huang, S.-Y.; Zou, X. Journal of computational chemistry 2006, 27, 1876-1882.

[51] Muegge, I.; Martin, Y. C. Journal of medicinal chemistry 1999, 42, 791-804.

[52] Huang, S.-Y.; Zou, X. Journal of Computational Chemistry 2006, 27, 1866-1875.

[53] Yan, C.; Zou, X. Computer-Aided Drug Discovery; Springer, 2015; pp 153-166.

[54] Guedes, I. A.; de Magalhães, C. S.; Dardenne, L. E. Biophysical Reviews 2013, $6,75-87$.

[55] Kitchen, D. B.; Decornez, H.; Furr, J. R.; Bajorath, J. Nature reviews Drug discovery 2004, 3, 935.

[56] Huang, H.; Zhang, G.; Zhou, Y.; Lin, C.; Chen, S.; Lin, Y.; Mai, S.; Huang, Z. Frontiers in Chemistry 2018, 6.

[57] Li, H.; Leung, K.-S.; Ballester, P. J.; Wong, M.-H. PLoS ONE 2014, 9, e85678. 
[58] Quiroga, R.; Villarreal, M. A. PLOS ONE 2016, 11, e0155183.

[59] Ferrara, P.; Gohlke, H.; Price, D. J.; Klebe, G.; Brooks, C. L. Journal of medicinal chemistry 2004, 47, 3032-3047.

[60] Li, Y.; Han, L.; Liu, Z.; Wang, R. Journal of Chemical Information and Modeling 2014, 54, 1717-1736.

[61] Xu, W.; Lucke, A. J.; Fairlie, D. P. Journal of Molecular Graphics and Modelling 2015, 57, 76-88.

[62] Huang, S.-Y. Briefings in bioinformatics 2017,

[63] Pagadala, N. S.; Syed, K.; Tuszynski, J. Biophysical Reviews 2017, 9, 91-102.

[64] Zavodszky, M. I.; Rohatgi, A.; Voorst, J. R. V.; Yan, H.; Kuhn, L. A. Journal of Molecular Recognition 2009, 22, 280-292.

[65] Wang, R.; Lai, L.; Wang, S. Journal of computer-aided molecular design 2002, $16,11-26$.

[66] Paul, N.; Rognan, D. Proteins: Structure, Function, and Genetics 2002, 47, $521-533$.

[67] Shin, W.-H.; Seok, C. Journal of Chemical Information and Modeling 2012, 52, $3225-3232$.

[68] Huang, S.-Y.; Zou, X. Proteins: Structure, Function, and Bioinformatics 2006, 66, 399-421. 
[69] Mizutani, M. Y.; Takamatsu, Y.; Ichinose, T.; Nakamura, K.; Itai, A. Proteins: Structure, Function, and Bioinformatics 2006, 63, 878-891.

[70] Bottegoni, G.; Kufareva, I.; Totrov, M.; Abagyan, R. Journal of Computer-Aided Molecular Design 2008, 22, 311-325.

[71] Nivedha, A. K.; Thieker, D. F.; Makeneni, S.; Hu, H.; Woods, R. J. Journal of Chemical Theory and Computation 2016, 12, 892-901.

[72] Yin, S.; Biedermannova, L.; Vondrasek, J.; Dokholyan, N. V. Journal of Chemical Information and Modeling 2008, 48, 1656-1662.

[73] Morris, G. M.; Goodsell, D. S.; Halliday, R. S.; Huey, R.; Hart, W. E.; Belew, R. K.; Olson, A. J. Journal of Computational Chemistry 1998, 19, 16391662.

[74] Ravindranath, P. A.; Forli, S.; Goodsell, D. S.; Olson, A. J.; Sanner, M. F. PLOS Computational Biology 2015, 11, e1004586.

[75] Koebel, M. R.; Schmadeke, G.; Posner, R. G.; Sirimulla, S. Journal of Cheminformatics 2016, 8 .

[76] Abagyan, R.; Totrov, M.; Kuznetsov, D. Journal of Computational Chemistry 1994, 15, 488-506.

[77] Krammer, A.; Kirchhoff, P. D.; Jiang, X.; Venkatachalam, C.; Waldman, M. Journal of Molecular Graphics and Modelling 2005, 23, 395-407.

[78] Trott, O.; Olson, A. J. Journal of computational chemistry 2010, 31, 455-461. 
[79] Kerzmann, A.; Fuhrmann, J.; Kohlbacher, O.; Neumann, D. Journal of Chemical Information and Modeling 2008, 48, 1616-1625.

[80] Hartmann, C.; Antes, I.; Lengauer, T. Proteins: Structure, Function, and Bioinformatics 2009, 74, 712-726.

[81] Malik, V.; Dhanjal, J. K.; Kumari, A.; Radhakrishnan, N.; Singh, K.; Sundar, D. Methods 2017, 131, 10-21.

[82] Xu, X.; Huang, M.; Zou, X. Biophysics Reports 2018, 4, 1-16.

[83] Zheng, M.; Liu, X.; Xu, Y.; Li, H.; Luo, C.; Jiang, H. Trends in Pharmacological Sciences 2013, 34, 549-559.

[84] Lauro, G.; Masullo, M.; Piacente, S.; Riccio, R.; Bifulco, G. Bioorganic 6 Medicinal Chemistry 2012, 20, 3596-3602.

[85] Lavecchia, A.; Di Giovanni, C. Current medicinal chemistry 2013, 20, 28392860.

[86] Tan, L.; Geppert, H.; Sisay, M.; Gtschow, M.; Bajorath, J. ChemMedChem 2008, 3, 1566-1571.

[87] Bullock, C.; Cornia, N.; Jacob, R.; Remm, A.; Peavey, T.; Weekes, K.; Mallory, C.; Oxford, J. T.; McDougal, O. M.; Andersen, T. L. Journal of Chemical Information and Modeling 2013, 53, 2161-2170.

[88] Keiser, M. J. et al. Nature 2009, 462, 175-181.

[89] Haupt, V. J.; Schroeder, M. Briefings in Bioinformatics 2011, 12, 312-326. 
[90] Nagaraj, A. B.; Wang, Q. Q.; Joseph, P.; Zheng, C.; Chen, Y.; Kovalenko, O.; Singh, S.; Armstrong, A.; Resnick, K.; Zanotti, K.; Waggoner, S.; Xu, R.; DiFeo, A. Oncogene 2017, 37, 403-414.

[91] Ma, Z.; Xu, X.; Zou, X. Biophysical Journal 2018, 114, 56a.

[92] Chen, H.; Engkvist, O.; Wang, Y.; Olivecrona, M.; Blaschke, T. Drug Discovery Today 2018, 23, 1241-1250.

[93] Russo, D. P.; Zorn, K. M.; Clark, A. M.; Zhu, H.; Ekins, S. Molecular pharmaceutics 2018, 15, 4361-4370.

[94] Samardzhieva, I.; Khan, A. International Journal of Computer Applications 2018, 182, 1-10.

[95] Chen, Y.-C. Trends in Pharmacological Sciences 2015, 36, 78-95.

[96] Carlson, H. A. Journal of Chemical Information and Modeling 2016, 56, 951954 .

[97] Gathiaka, S.; Liu, S.; Chiu, M.; Yang, H.; Stuckey, J. A.; Kang, Y. N.; Delproposto, J.; Kubish, G.; Dunbar, J. B.; Carlson, H. A.; Burley, S. K.; Walters, W. P.; Amaro, R. E.; Feher, V. A.; Gilson, M. K. Journal of Computer-Aided Molecular Design 2016, 30, 651-668.

[98] Gaieb, Z.; Liu, S.; Gathiaka, S.; Chiu, M.; Yang, H.; Shao, C.; Feher, V. A.; Walters, W. P.; Kuhn, B.; Rudolph, M. G.; Burley, S. K.; Gilson, M. K.; Amaro, R. E. Journal of Computer-Aided Molecular Design 2017, 32, 1-20. 
[99] Gaieb, Z.; Parks, C. D.; Chiu, M.; Yang, H.; Shao, C.; Walters, W. P.; Lambert, M. H.; Nevins, N.; Bembenek, S. D.; Ameriks, M. K.; Mirzadegan, T.; Burley, S. K.; Amaro, R. E.; Gilson, M. K. Journal of Computer-Aided Molecular Design 2019, 33, 1-18.

[100] Wagner, J. R.; Churas, C. P.; Liu, S.; Swift, R. V.; Chiu, M.; Shao, C.; Feher, V. A.; Burley, S. K.; Gilson, M. K.; Amaro, R. E. 2018,

[101] Xu, X.; Ma, Z.; Duan, R.; Zou, X. Journal of Computer-Aided Molecular Design 2019, 33, 367-374. 


\title{
Chapter 2
}

\section{Drug Discovery against Diverse Kinases: Predicting Selectivity and Virtual Screening}

\begin{abstract}
Docking against multiple target proteins is necessary for the prediction of ligand specificity and for rapid identification of inhibitors within a family of proteins. However, sequentially docking ligands into each target using conventional single-target docking methods is computationally expensive for virtual ligand screening, especially with a large number of the targets. This work introduced an efficient ensemble docking algorithm to address the issue of simultaneous docking against multiple targets. We used 14 protein kinases to study ligand selectivity and employed staurosporine and Gleevec for the representation of nonselective and selective binding. Virtual ligand screening was also performed against five protein kinases that have at least seven known inhibitors; the enrichments for ensemble docking were shown to be comparable
\end{abstract}


to those for sequentially docking the ligands to multiple targets. We demonstrated the ensemble docking algorithm to be computationally efficient, with a run time comparable to that of single-target docking. Our method will save significant amounts of computational time for studies of ligand selectivity in structure-based drug design.

\subsection{Introduction}

Molecular docking has become a common tool in structure-based drug design (see refs $^{1-6}$ for review). Particularly, ligand-protein docking, which addresses the likely binding modes of a ligand interacting with a protein of known 3D structure, is extensively applied to a variety of work in modern drug discovery. ${ }^{7,8}$ However, there are so many opportunities for promiscuity due to both similarities of cognate protein structures and flexibilities in the active binding sites when protein and ligands are cross-docked, resulting in possible side effects of drugs. An exemplary example is protein kinases.

As one of the largest families of enzymes, protein kinases are crucial in signal transduction cascades, regulating protein functions by chemically adding a negativelycharged phosphate group. Protein kinases are important targets for cancers and inflammation. ${ }^{9-11}$ Consequently, numerous efforts have been devoted to developing kinase inhibitors. In the past decade alone, more than 20 drugs targeting protein kinases were approved for clinical use. ${ }^{12}$ Despite the achieved success, the broad promiscuity causing undesirable off-target actions and side effects remains a major problem for protein kinase inhibitors. Both the presence of hundreds of protein kinases and the highly conserved residues in the active binding sites make it difficult for target 
specificity. Therefore, ligand selectivity is crucial yet challenging for the development of kinase-targeted therapies.

Most current docking programs are designed for a single protein target, meaning that only one receptor can be considered in a docking process. This is a reasonable restriction in most cases, because one is often interested in a single, specific protein target at a time. However, investigating the selectivity of an inhibitor with multiple protein targets requires a more advanced method. ${ }^{16-19}$ Current docking programs sequentially dock ligands into each of the target proteins, a process referred to as sequential docking. The results from each single-target docking merge together via re-ranking. Despite its feasibility, this kind of sequential docking demands extensive computational investment, with the computational time being proportional to the number of the target proteins involved. In addition, sequential docking also results in added inconvenience during docking setup and the analysis of docking results. Therefore, a docking strategy that can simultaneously address multiple targets would be highly preferred.

This work presents an efficient ensemble docking algorithm that can simultaneously dock ligands into multiple target proteins without significantly increasing the computational time. The ensemble docking algorithm regards the protein structure as an additional parameter in the energy optimization process and performs docking against multiple targets like docking against a single target. After docking calculations, each ligand receives a series of energy scores corresponding to different target proteins so that one can investigate the selectivity of the ligand against different targets. The binding scores also yield multiple enrichment curves corresponding to different targets in the same virtual screening experiment. We use protein kinases as 
a test case because they share similar binding pockets that provide an excellent test system and because they have important clinical applicaitons. Our ensemble docking algorithm performs well on ligand selectivity and virtual screening of large compound database. We also employ our docking algorithm on Gleevec to identify specificity and achieve good agreement with the reference study.

\subsection{Materials and Methods}

\subsubsection{Ensemble docking algorithm to simultaneously address multiple targets}

The main purpose of our ensemble algorithm is to introduce an additional parameter in docking calculations used to describe different protein structures. In traditional single-rigid-receptor docking software such as DOCK,${ }^{20}$ the receptor is fixed and the ligand is allowed to orient in the binding site. The binding energy score between the ligand and the receptor can be expressed as

$$
E^{\mathrm{S}}=E(x, y, z, \theta, \phi, \psi)
$$

where $x, y$, and $z$ represent the coordinates of the center of mass in the ligand, and $\theta, \phi$, and $\psi$ denote the three Euler angles, respectively. These six parameters define the orientation of the ligand. The binding score is only determined by the orientation of the ligand. The optimization procedure of docking involves continuous adjustment $(x, y, z, \theta, \phi, \psi)$ until the calculated score converges to the minimum, corresponding to the predicted binding mode. Therefore, if a ligand needs to be docked into an 
ensemble of $M$ target proteins by using the standard docking approach, the docking procedure needs to be performed $M$ times. In addition, the computational time will be $M$ times that of the CPU for docking against a single target.

In order to improve the efficiency of docking against multiple targets, our ensemble docking algorithm adds the number of protein targets as the seventh dimension for scoring. Specifically, the ensemble algorithm reconsiders the scoring procedure as

$$
E^{\mathrm{M}}=E(x, y, z, \theta, \phi, \psi, m)
$$

where $m$ stands for the $m$-th protein of the target ensemble, an integer parameter ranging from 1 to $M$. Namely, in our ensemble docking algorithm, the binding score depends not only on the ligand orientation (determined by $x, y, z, \theta, \phi, \psi)$ but also the specific target protein (denoted by $m$ ). Thus, by adjusting the parameter set $(x, y, z, \theta, \phi, \psi, m)$, our ensemble algorithm can simultaneously dock a ligand into the ensemble of multiple target proteins.

When only one target protein exists, i.e. $M=1$, ensemble docking by eq (2.2) reverts to standard single-target docking by eq (2.1). Similar ensemble algorithms have

been used to consider protein structural variations in ligand binding. ${ }^{21,22}$ It has been demonstrated that the computational time of our ensemble docking is comparable to that of single-target docking.

\subsubsection{Scoring function}

The scoring function that used in this study is ITScore, an accurate iterative knowledgebased scoring function developed by our group. ${ }^{23,24}$ The energy score between protein 
and ligand $E_{\mathrm{P}-\mathrm{L}}$ is obtained by summing up all protein(P)-ligand(L) atom pair interactions as

$$
E_{\mathrm{P}-\mathrm{L}}=\sum_{\mathrm{P}-\mathrm{L} \text { atom pair }} u_{i j}(r)
$$

where $u_{i j}(r)$ is the pair potential of ITScore between the protein atom of type $i$ and the ligand atom of type $j$ at the atom pair distance $r$. Only intermolecular interactions are considered in ITScore. In this work, the scoring fucntion was derived via an effcient iterative method using 2897 protein-ligand complexes extracted from

the Protein Data Bank (PDB). ${ }^{25}$ Its efficiency and versatility were demonstrated through binding mode identification, binding affinity prediction, and virtual database screening on diverse test sets of up to 200 protein-ligand complexes. Details of the theory of ITScore and related test studies were described in our previous studies. ${ }^{23,24}$ In the present work, the protein is treated as a rigid body; the ligand is allowed to be flexible in docking calculations. The ligand flexibility is represented by multiple conformers, which are generated by the OMEGA software (version 2.4.6. OpenEye Scientific Software, Santa Fe, NM. http://www.eyesopen.com.) $)^{26,27}$

\subsubsection{Construction of the reference protein}

Our docking program uses the same matching algorithm as the DOCK 4.0 package. ${ }^{20,28}$ Therefore, similar to DOCK 4.0, a protein structure is required to guide the generation of initial ligand orientations around the binding site. However, our ensemble docking algorithm works on multiple target proteins, and it is neither possible nor reasonable to determine which target should be used as a representative of the ensemble. Therefore, a reference protein will be automatically constructed by the 
docking program for generation of initial ligand orientations.

The principle for constructing the reference protein is the same as that in the previous work. ${ }^{21}$ Namely, the binding pocket should be as large as possible to provide sufficient sampling space for the ligand with the least change in the overall shape of the binding site. The details are described as follows.

First, by collecting and clustering the sphere points of the ensemble, we generated a set of reference sphere points. ${ }^{21}$ We calculated the distances between the reference sphere points and each residue of every target protein, with the distance defined as the minimum of the distances between every atom in the residue and any of the reference sphere points. Then, we performed multiple sequence alignment for multiple target proteins by using the structure-based sequence alignment software T-Coffee, ${ }^{29}$ and randomly selected one of the multiple sequences to be the reference sequence of the reference protein. Then, the conformation of a residue was chosen as a representation of the corresponding residue of the reference sequence by satisfying the following two conditions: the residue of the selected conformation was aligned with the residue of the reference sequence within at least $3 \AA$ from the reference sphere points, and all the selected residue conformations were finally combined into the reference protein.

It should be noted that the constructed reference protein is artificial. However, this is irrelevant because the reference protein only guides the generation of initial ligand orientations. The real target proteins, rather than the reference protein, will be used for score calculations and orientation optimization. 


\subsubsection{Docking algorithm}

We wrote a Fortran docking program to implement our ensemble docking algorithm ${ }^{21,22}$ by using the ligand matching method of DOCK $4.0^{28}$ and the ITScore scoring function, ${ }^{23,24}$ named MDock (c) University of Missouri-Columbia, 2007). We re-trained the scoring function using 2897 protein-ligand complexes from the Protein Data Bank. The ligand flexibility is considered by docking multiple conformers of a ligand generated by the OMEGA software under default parameters. The maximum number of output conformers per ligand, MAXCONFS, was set to the recommended values (200).

The initial ligand orientations were generated by using the exhaustive matching algorithm by Ewing et al., ${ }^{30}$ which is guided by a reference protein of multiple targets. About 50 sphere points represented the binding site. The tolerance distance between ligand atoms and sphere points was set to $0.5 \AA$ during matching. The ligand orientation that matched the most sphere points underwent further scoring evaluation, with the maximum number of evaluated orientations set to 1000, and the maximum minimization step set to 200. Equation (2.2) calculated ligand energy scores for our ensemble docking algorithm. By employing the grid-based energy calculation algorithm of DOCK ${ }^{31}$ we improved the computational speed. The grid spacing was set to $0.3 \AA$, and trilinear interpolation was used to calculate energy scores. SIMPLEX optimized the binding energy of each ligand orientation. ${ }^{32}$ Because SIMPLEX is essentially a local optimization method, the top 20 orientations for each ligand were re-optimized according to each target protein as a way to reduce the influence of local minima. We saved the ligand binding scores of each target protein for sequent analysis. In short, we have $M$ sets of energy scores for each ligand corresponding to 
different target proteins of the ensemble.

\subsubsection{Test data sets}

The protein kinases obtained from the PdbBind (2015 version) database were selected as a test set because the experimental binding affinity data of the complexes were available in this database. ${ }^{33,34}$ We extracted 200 kinase complexes from a collection of 2401 hits in the PdbBind database by applying guidelines as follows: (1) only human gene protein kinases; (2) pH ranges from 6.8 to 7.5; (3) not binding with ATP, ADP, ANP or peptide; (4) X-ray structures rather than NMR models. These 200 protein kinase complexes belong to 14 different protein kinases (see Table 2.1) and were used to evaluated our docking algorithm and scoring function by performing traditional single-target docking (see Supporting Information). The 200 ligands served as the active compounds in the virtual screening test.

Each of the 14 protein kinases includes a certain number of complexes. Each protein kinase was represented by one protein structure with high resolution and no missing residue in its binding site. Thus, we obtained a total of 14 target proteins representing 14 protein kinases (see Table 2.1), which will be used to evaluate our ensemble docking algorithm.

The Protein Data Bank provided coordinates for all the complexes. ${ }^{25}$ The multiProt software superimposed the 14 target proteins representing 14 protein kinases according to their binding sites. ${ }^{35}$ Water molecules and metal ions were removed from the protein structures. Hydrogen atoms were not considered explicitly because of the feature of the ITScore scoring function we used in this study. ${ }^{23,24} \mathrm{~T}$-Coffee software ${ }^{29}$ aligned the sequences of 14 target proteins for construction of the reference 
protein. SYBYL software assigned atom types to the proteins and ligands(Tripos, Inc.). During docking calculations, proteins were treated as rigid bodies and ligands were allowed to be flexible.

\subsubsection{Database preparation}

For a small-scale virtual screening test, 1000 commercially available compounds were randomly selected from the Available Chemical Directory (ACD, distributed by Molecular Design Ltd., San Leandro, CA) with the aid of the FILTER program (OpenEye Inc.) under default parameters . These 1000 molecules served as a set of inactive compounds for virtual database screening. The CONCORD program generated coordinates for the molecules. ${ }^{36}$ 
Table 2.1: The 14 protein kinases for testing the ensemble docking algorithm. The PDB codes of corresponding ligand-bound complexes are listed in the right column with the ligand IDs in parentheses. The protein structure with bold font represents its corresponding protein kinase in ensemble docking calculations.

\begin{tabular}{|c|c|}
\hline Kinases & Protein-ligand Complexes \\
\hline P38 & 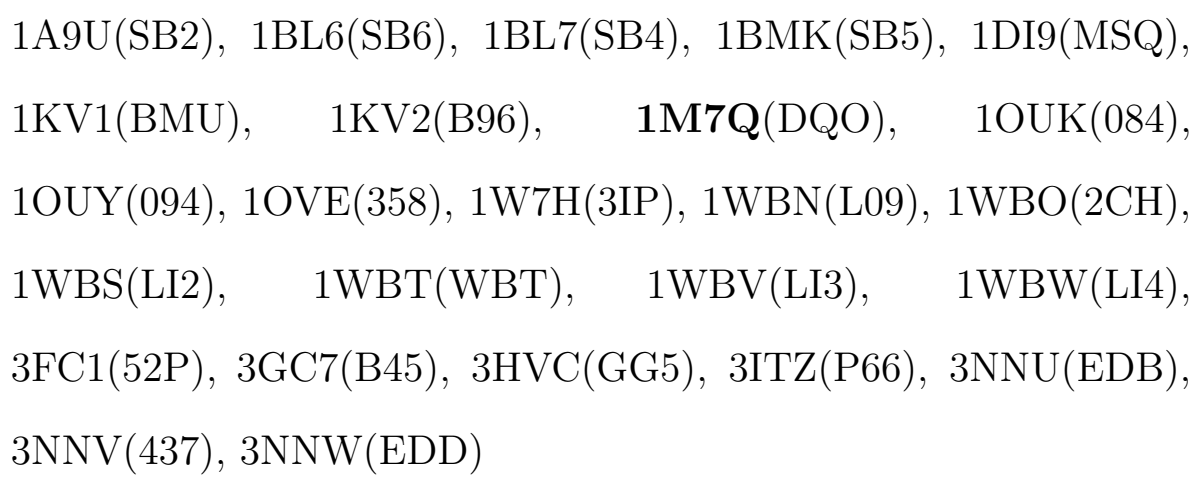 \\
\hline TGFR & $\begin{array}{l}\text { 1PY5(PY1), 1RW8(580), 2X7O(ZOP), 3FAA(55F), 3KCF(JZO), } \\
\text { 4L3P(1UH), 4L52(1UL), 4L53(1UO), 4O91(NG2) }\end{array}$ \\
\hline PDK1 & $\begin{array}{l}\text { 1OKY(STU), 1OKZ(UCN), 1UU3(LY4), 1UU7(BI2), 1UU8(BI1), } \\
\text { 1UVR(BI8), 1Z5M(LI8), 3QC4(MP7), 3RCJ(3RC), 3RWP(ABQ), } \\
\text { 3RWQ(3RW), 3SC1(3S1) }\end{array}$ \\
\hline
\end{tabular}




\begin{tabular}{|c|c|}
\hline CDK2 & 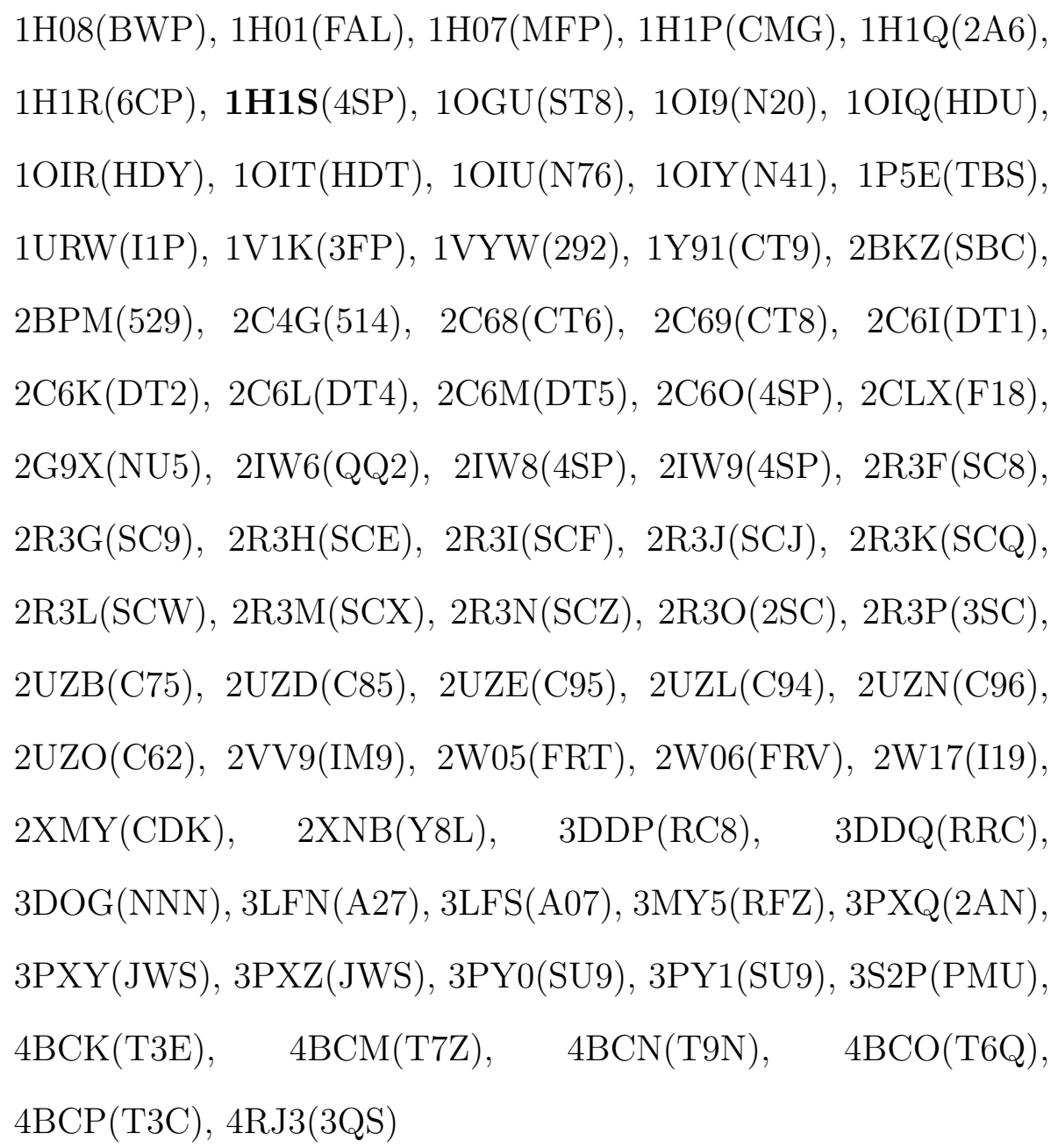 \\
\hline AURKA & $\begin{array}{l}\text { 3P9J }(\mathrm{P} 9 J), \quad 4 \mathrm{DEA}(\mathrm{NHI}) \\
4 \mathrm{UZD}(\mathrm{QMN})\end{array}$ \\
\hline CHK1 & $\begin{array}{l}2 \mathrm{HOG}(710), 2 \mathrm{HXL}(422), 2 \mathrm{HXQ}(373), 2 \mathrm{HY0}(306), 2 \mathrm{QHM}(7 \mathrm{CS}), \\
2 \mathrm{QHN}(582), 2 \mathrm{R} 0 \mathrm{U}(\mathrm{M} 54), 2 \mathrm{X} 8 \mathrm{D}(\mathrm{X} 8 \mathrm{D}), 3 \mathrm{TKH}(07 \mathrm{~S}), 3 \mathrm{TKI}(\mathrm{S} 25)\end{array}$ \\
\hline CK2 & 3MB6(01I), 3MB7(14I) \\
\hline
\end{tabular}




\begin{tabular}{|c|c|}
\hline JNK3 & $\begin{array}{l}\text { 1PMN(984), 2B1P(AIZ), 2EXC(JNK), 3G90(J72), 3G9L(J67), } \\
\text { 3G9N(j88), 3OY1(589), 3V6R(CQQ), 3V6S(0F0), 4U79(3EL), } \\
\text { 4WHZ(3NL) }\end{array}$ \\
\hline JAK2 & $\begin{array}{l}\text { 3E62(5B1), 3E63(5B2), 3E64(5B3), 3JY9(JZH), 3KCK(3KC), } \\
\text { 3KRR(DQX), 3Q32(J2I), 3RVG(17P), 3ZMM(F9J), 4BBE(3O4), } \\
4 \mathrm{BBF}(\mathrm{O} 19), 4 \mathrm{C} 61(\mathrm{LMM}), 4 \mathrm{D} 0 \mathrm{~W}(\mathrm{VVQ}), 4 \mathrm{D} 0 \mathrm{X}(953)\end{array}$ \\
\hline $\mathrm{HGF}$ & $\begin{array}{l}\text { 1R0P(KSA), 3CE3(1FN), 3CTH(319), 3CTJ(320), 3F82(353), } \\
\text { 3Q6W(Q6W), 3QTI(3QT), 3R7O(M61), 4EEV(L1X), 4GG5(0J3), } \\
\text { 4GG7(0J8), 4IWD(1JC) }\end{array}$ \\
\hline ERK2 & $\begin{array}{l}\text { 1PME(577), 2OJG(19A), 2OJI(33A), 2OJJ(82A), 3I5Z(Z48), } \\
\text { 3I60(E86), 4O6E(2SH), 4QTA(38Z) }\end{array}$ \\
\hline GSK $3 \beta$ & $\begin{array}{l}\text { 1Q3D(STU), 1Q3W(ATU), 1Q41(679), 3GB2(G3B), 3I4B(Z48), } \\
\text { 3L1S(Z92), 4IQ6(IQ6) }\end{array}$ \\
\hline ITK & $\begin{array}{l}\text { 3T9T(IAQ), 4HCT(18R), 4HCU(13L), 4HCV(13J), 4M0Y(M0Y), } \\
\text { 4M0Z(M0Z), 4M14(QWS) }\end{array}$ \\
\hline PAK1 & 4EQC(XR1), 4O0R(X4Z), 4O0T(2OL) \\
\hline
\end{tabular}


Table 2.2: A ranked list of 14 protein kinases according to the computed binding energies by ensemble docking experiments for seven typical inhitors. The true targets of each inhibitor are colored in gray.

\begin{tabular}{|cc|cc|cc|cc|cc|cc|cc|}
\hline \multicolumn{2}{|c|}{ I17 } & \multicolumn{2}{|c|}{ MNY } & \multicolumn{2}{c|}{ KSA } & \multicolumn{2}{c|}{ TBS } & \multicolumn{2}{c|}{ STU } & \multicolumn{2}{c|}{084} & \multicolumn{2}{c|}{460} \\
\hline Kinase & Score & Kinase & Score & Kinase & Score & Kinase & Score & Kinase & Score & Kinase & Score & Kinase & Score \\
\hline CDK2 & -51.6 & CK2 & -45.3 & HGF & -66.8 & CK2 & -49.2 & PDK1 & -52.7 & P38 & -51.4 & TGFR & -47.1 \\
HGF & -49.9 & JAK2 & -43.5 & PDK1 & -51.5 & CDK2 & -49.0 & CDK2 & -48.5 & JNK3 & -47.3 & JNK3 & -46.0 \\
CHK1 & -47.2 & CDK2 & -41.9 & CK2 & -51.3 & ERK2 & -47.4 & CHK1 & -45.4 & ERK2 & -43.2 & HGF & -43.7 \\
JNK3 & -45.6 & HGF & -41.4 & CHK1 & -46.8 & HGF & -45.6 & GSK3 $\beta$ & -45.0 & AURKA & -43.2 & CK2 & -41.7 \\
ERK2 & -45.2 & CHK1 & -41.2 & TGFR & -44.6 & JNK3 & -44.9 & JNK3 & -41.5 & TGFR & -41.9 & ERK2 & -41.5 \\
CK2 & -43.9 & JNK3 & -40.3 & JNK3 & -44.5 & JAK2 & -44.2 & HGF & 40.8 & CK2 & -40.4 & PDK1 & -38.4 \\
AURKA & -40.6 & AURKA & -38.8 & AURKA & -42.4 & PDK1 & -43.4 & ERK2 & -40.7 & PAK1 & -40.1 & AURKA & -38.2 \\
ITK & -39.6 & PDK1 & -37.7 & GSK3 $\beta$ & -41.5 & AURKA & -42.7 & CK2 & -40.1 & GSK3 $\beta$ & -40.1 & ITK & -38.2 \\
JAK2 & -39.1 & TGFR & -37.7 & ERK2 & -40.6 & CHK1 & -42.2 & AURKA & -38.8 & PDK1 & -40.0 & CHK1 & -37.0 \\
PDK1 & -39.1 & ERK2 & -36.3 & ITK & -38.3 & ITK & -41.9 & P38 & -34.4 & CDK2 & -40.0 & GSK3 3 & -34.3 \\
P38 & -39.1 & ITK & -35.8 & JAK2 & -35.4 & P38 & -41.6 & ITK & -30.7 & CHK1 & -40.0 & P38 & -33.4 \\
GSK3 $\beta$ & -39.0 & P38 & -35.2 & PAK1 & -35.3 & PAK1 & -41.6 & PAK1 & -30.3 & HGF & -38.9 & CDK2 & -33.2 \\
TGFR & -38.1 & PAK1 & -35.2 & P38 & -35.2 & TGFR & -41.5 & TGFR & -29.5 & ITK & -38.1 & PAK1 & -32.5 \\
PAK1 & -35.9 & GSK3 $\beta$ & -35.2 & CDK2 & -35.1 & GSK3 $\beta$ & -41.1 & JAK2 & -28.9 & JAK2 & -34.2 & JAK2 & -32.5 \\
\hline
\end{tabular}

\section{$2.3 \quad$ Results}

One major limitation of standard single-target docking against multiple targets is its lack of efficiency in a large database screening, especially for a large number of the targets. We have performed virtual database screening against multiple target proteins to evaluate our ensemble docking algorithm. The test was performed on an ensemble of 14 protein kinases (see Materials and Method). The results will be compared with those of standard single-target docking. 


\subsubsection{Evaluation of the dock algorithm and scoring function}

Before docking against the ensemble of multiple target proteins, we first re-docked the 200 ligands into their bound protein structures (Table 1), referred as native docking, which served as validation of our docking algorithm and scoring function.

In the present work, the docking accuracy was evaluated by calculating the rootmean-square deviation (rmsd) between the docked ligand and the ligand in the crystal complex structure. The ligand binding mode was considered to be successfully predicted if the rmsd $<2.0 \AA$ for the top orientation. ${ }^{37}$ This represents the default criterion unless otherwise specified. Figure 2.1 shows the success rates of our docking algorithm for 200 ligands with different criteria ranging from rmsd $<1.0 \AA$ to rmsd $<3.0 \AA$. From this figure, we can see that our docking algorithm yields high success rates at different rmsd criteria. Under the strictest criterion, rmsd $<1.0 \AA$, the lowest success rate achieves $74 \%$. Under looser restrictions the success rate increases. The highest success rate, $83 \%$, resulted from rmsd $<3.0$. The default criterion of rmsd $<2.0 \AA$, yielded a success rate of $79 \%$. The high success rates validated our docking algorithm and scoring function. 


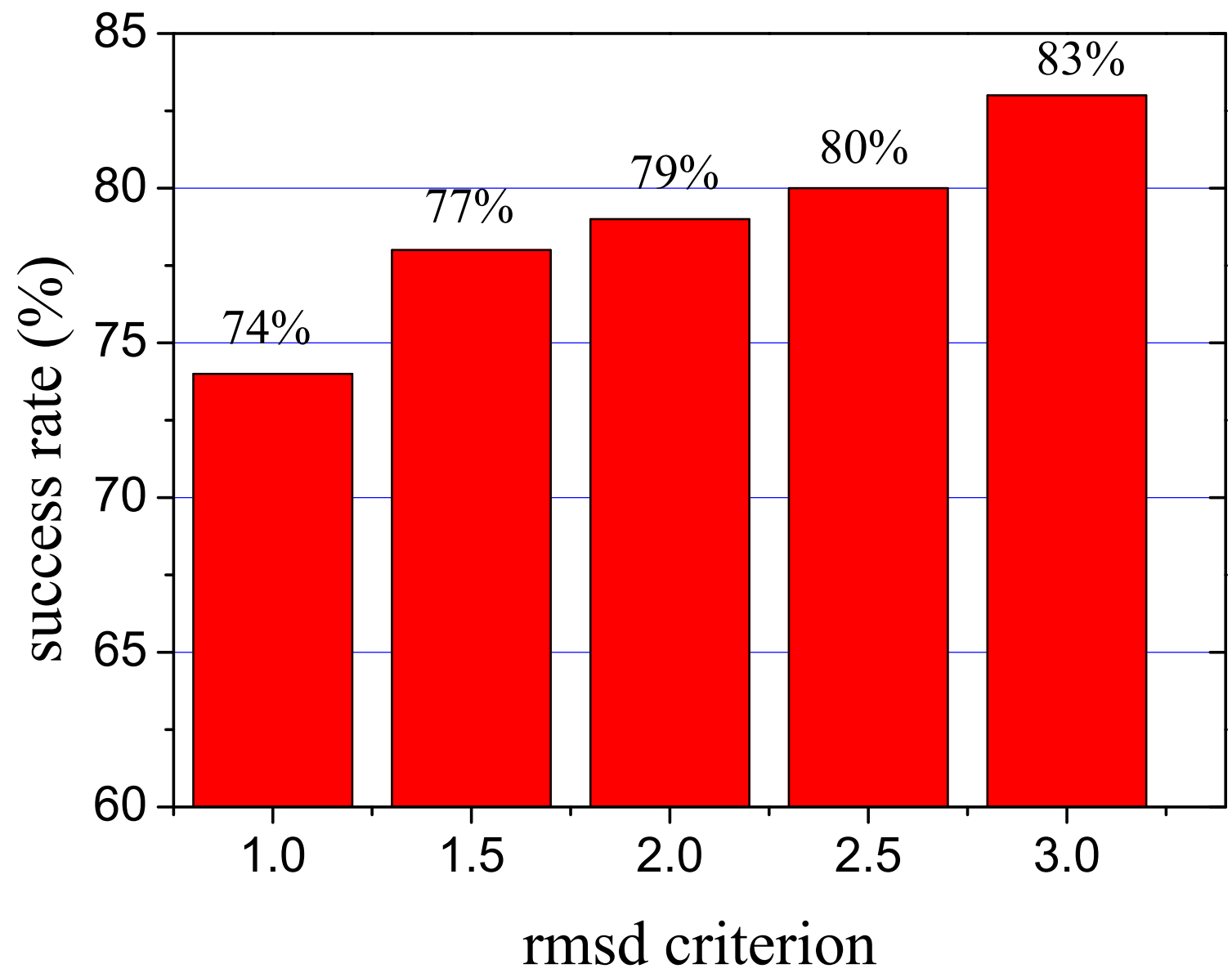

Figure 2.1: The success rates in binding mode predictions for 200 complexes at different criteria when the top conformation was considered: rmsd $<1.0,1.5,2.0,2.5$ and $3.0 \AA$, respectively.

\subsubsection{The ligand selectivity}

Another task of the ensemble docking algorithm is to investigate the ligand selectivity against different target proteins. Ideally, a docking algorithm would give the same energy scores as experimental binding affinity for those targets of an inhibitor and worse scores for those non-targets. However, our scoring function was not scaled to 


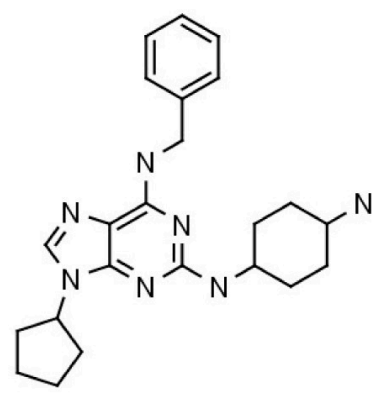

1G5S_I17<smiles>Nc1ccc(N)c2c1C(=O)c1c(O)ccc(O)c1C2=O</smiles>

1M2R_MNY

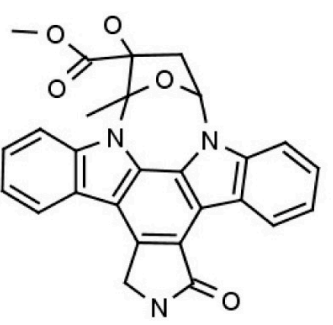

1R0P_KSA<smiles>Cc1cccc(C2=C(c3ccc4ncccc4n3)CNN2)n1</smiles>

Figure 2.2: The 2D structures of selected protein kinase inhibitors for ligand selectivity analysis. The ligand IDs with their corresponding PDB codes are shown in the lower-left corner of each cell. This figure is drawn with Marvinsketch, ChemAxon Ltd., http://www.chemaxon.com/. 
reproduce experimental binding affinities, even though it obtain a good correlation between the calculated energy scores and experimental binding affinities. ${ }^{23,24}$ Therefore, we used an alternative ranking method to investigate the selectivity of the ligands against different protein kinases.

The principle of the ranking method is that the true targets for an inhibitor should have a more favorable ranking than those non-targets in terms of calculated binding scores. For an inhibitor, the proteins were only divided into two simple classes of targets and non-targets according to the available experimental data. Table 2.2 shows the ranked list of different protein kinases according to their binding scores for seven typical inhibitors (Figure 2.2 and Figure 2.3A ). From the table we can see that the true targets of the inhibitors indeed obtain better rankings than those of the non-targets. It is also notable that our ensemble docking algorithm identifies the targets of some inhibitors such as I17, MNY, 460 etc., even though the protein kinase ensemble did not include the bound protein structures of these inhibitors.

To further discuss our ensemble algorithm in ligand selectivity which is applicable for both non-specific and specific targets, we take an example of staurosporine (STU) and Gleevec (STI) to illustrate. For non-specific binding, staurosporine represent a well-studied class which has been used as a universal kinase inhibitor. ${ }^{38,39}$ The consistent conformations of protein kinases as well as the almost rigid structure with few rotatable bonds of staurosporine contribute to the similar binding sites conformations when staurosporine docking against protein kinase, including two important motifs (the GXGXXG motif and the DFG motif, see Figure 2.3A, 3C). Table 2.2 shows that STU inhibit at least 4 sub-families of protein kinases, and we obtained better rankings for these 4 targets from the 14 kinases in our ensemble docking. 
Comparing with promiscuity of staurosporine, a potent and effective drug Gleevec (also known as Imatinib or STI-571, Figure 2.3D), has been utilized as a primary clinical drug for treating certain cancers, such as chronic myelogenous leukemia (CML) and gastrointestinal stromal tumors (GISTs). ${ }^{40,41}$ Gleevec exhibits an effective autoinhibitory function specifically against c-Kit and Abl protein tyrosine kinase. In the tumor cell, tyrosine kinases are constitutively active, thus Gleevec is used to block kinase in an inactive conformation to preventing the phosphorylation that would benefit the cancerous cell. However, the reason why Gleevec is a specific and potent inhibitor has been controversial. ${ }^{42-44}$ Recently, Agafonov et al. ${ }^{45}$ experimentally identified that Gleevec specifically inhibitor of Abl rather than Src because of the induced-fit conformational change. They used NMR and fluorescence to directly detect Gleevec binding and gave a convinced explaination in their paper. In Figure 2.3D, we made an alignment of the 4 tyrosine kinase complexes (PDB ID as shown in Table 2.3) and noted by different color to focus on the conformational change after Gleevec binding to kinases. In the Figure 2.3D, Gleevec bind between $\alpha$ C-helix and the activation loop (A-loop) with Asp-Phe-Gly (DFG) motif, as the phosphate-binding loop (P-loop) which is responsible for stabilizing Gleevec binding. ${ }^{43}$ Although these 4 tyrosine kinases are homologous, they exhibit distinct conformations of the binding sites, which result in the binding specificity of Gleevec.

Our ensemble docking results on binding score of Gleevec with protein kinase yielding -76.8 and -72.8 for c-Kit and Abl, respectively. However, the energy scores are only -65.7 and -64.9 for Lck and c-Src, respectively (see Table 2.3). The energy scores exhibit sensitivity when Gleevec binding to Abl and c-Kit kinases, even including Lck and c-Src which are closely homologous tyrosine kinases of target proteins. Thus, the 
present results support the conclusions about effectiveness of our ensemble docking algorithm.

To quantitatively evaluate the efficiency of our ensemble docking algorithm in discriminating targets from non-targets, we employed a similar measure to that included in previous work done by our group: ${ }^{21}$

$$
E F=100 \% \times \frac{\langle\operatorname{Rank}(\text { nontarget })\rangle-\langle\operatorname{Rank}(\text { target })\rangle+N_{\text {proteins }} / 2}{N_{\text {proteins }}}
$$

where $N_{\text {proteins }}(=14$, here $)$ is the number of the proteins in the ensemble including both non-targets and targets, and $\langle\operatorname{Rank}($ nontarget $)\rangle$ and $\langle\operatorname{Rank}(\operatorname{target})\rangle$ denote the average ranking of the non-target and target proteins, respectively. The value of the efficiency factor $E F$ can vary from $0 \%$ to $100 \%$. If all the targets rank better than the non-targets, the efficiency factor $E F$ will be $100 \%$, indicating the complete agreement between the predictions and the experimental findings. In contrast, if all the non-targets rank better than the targets, the efficiency factor $E F$ will be $0 \%$. An equal distribution of the targets in the ranked list of proteins gives an efficiency factor of $50 \%$, representing random selection. The higher the $E F$ value, the better the efficiency of recovering known targets. Figure 2.4 gives the efficiency factors for all the ligands in identifying the true protein target by the ensemble docking algorithm. It can be seen from the figure that most of the ligands resulted in an efficiency factor of $>50 \%$ with an average efficiency of $74 \%$, indicating favorable rank for the true target over the nontargets. The overall good results demonstrate effectiveness of our ensemble algorithm in investigating the selectivity of inhibitors against multiple target proteins. 
Table 2.3: The energy scores of Gleevec binding to 4 homologous tyrosine kinases and to the 14 protein kinases in our ensemble docking test set. The true targets are colored gray.

\begin{tabular}{llll}
\hline Kinase & Score & Kinase & Score \\
c-Kit(1T46) & -76.8 & CHK1 & -50.3 \\
Abl(1IEP) & -72.8 & AURKA & -50.2 \\
Lck(2PL0) & -65.7 & GSK3 $\beta$ & -49.0 \\
c-Src(2OIQ) & -64.9 & TGFR & -48.9 \\
JNK3 & -53.9 & ERK2 & -47.7 \\
CDK2 & -52.0 & JAK2 & -47.6 \\
ITK & -51.8 & P38 & -47.3 \\
HGF & -51.6 & PDK1 & -47.3 \\
CK2 & -51.2 & PAK1 & -46.4 \\
\hline
\end{tabular}

\subsubsection{Virtual Database Screening}

A widely-used index for success in virtual screening is the enrichment factor, defined as the accumulated rate of known inhibitors identified in a certain top percentage of the ranked database. As described in the Materials and Methods section, our ensemble docking algorithm can give the binding energy scores of a ligand for each 


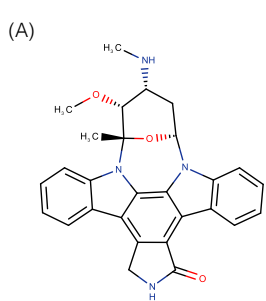

Staurosporine

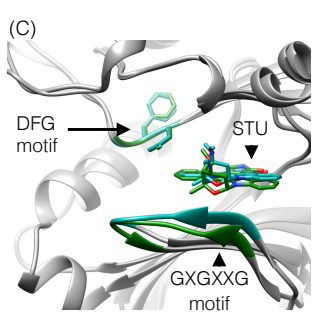

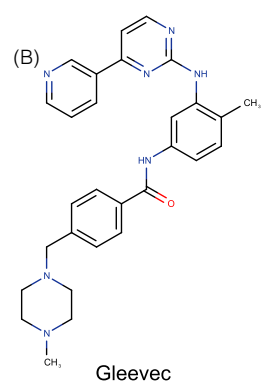

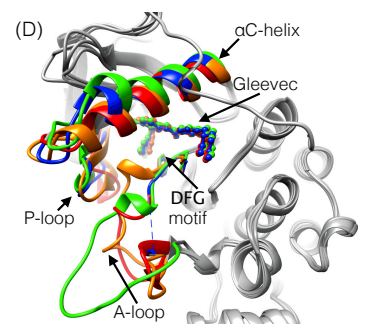

Figure 2.3: (A) The 2D structures of staurosporine. (B) The 2D structures of Gleevec. (C) The binding pocket conformations of PDK1 kinase (sea green) and GSK3 $\beta$ (forest green) bound with staurosporine. (D) Superimposition of the conformation of Gleevec bound to the Tyrosine Kinase domains of Abl(red), c-Kit(orange), Lck(green), and c-Src(blue). Gleevec is shown in the ball \& stick representation. 
of the targets in a docking calculation. Namely, each target protein will have a set of binding energy scores for all the docked ligands. Therefore, we can obtain multiple enrichment curves corresponding to multiple target proteins in only one ensemble docking calculation. However, one question remains: how does the accuracy of the docking results differ from that of the ensemble docking algorithm? To answer this question, we compared the enrichment data from the ensemble docking algorithm with those from the standard single-target docking method. Figure 2.5 compares the enrichment data between ensemble docking of multiple targets and standard singletarget docking on five typical target proteins with at least seven known ligands (Table 2.1). It can be seen from the figure that ensemble docking performs comparably well to standard single-target docking for all the target proteins, indicating that our ensemble docking algorithm efficiently identifies known ligands.

\subsubsection{Computational efficiency}

In addition to docking accuracy, another advantage of our ensemble docking algorithm is its superior computational efficiency. As demonstrated previously, the run time of ensemble docking algorithm is comparable to that of single-structure docking, though the ensemble algorithm addresses multiple protein structures. ${ }^{21,22}$ In the present work, the ensemble docking algorithm only needs on average 2.8 seconds to place and optimize a ligand conformer in the binding site, another result comparable to that of standard single-target docking (2.4 seconds). 


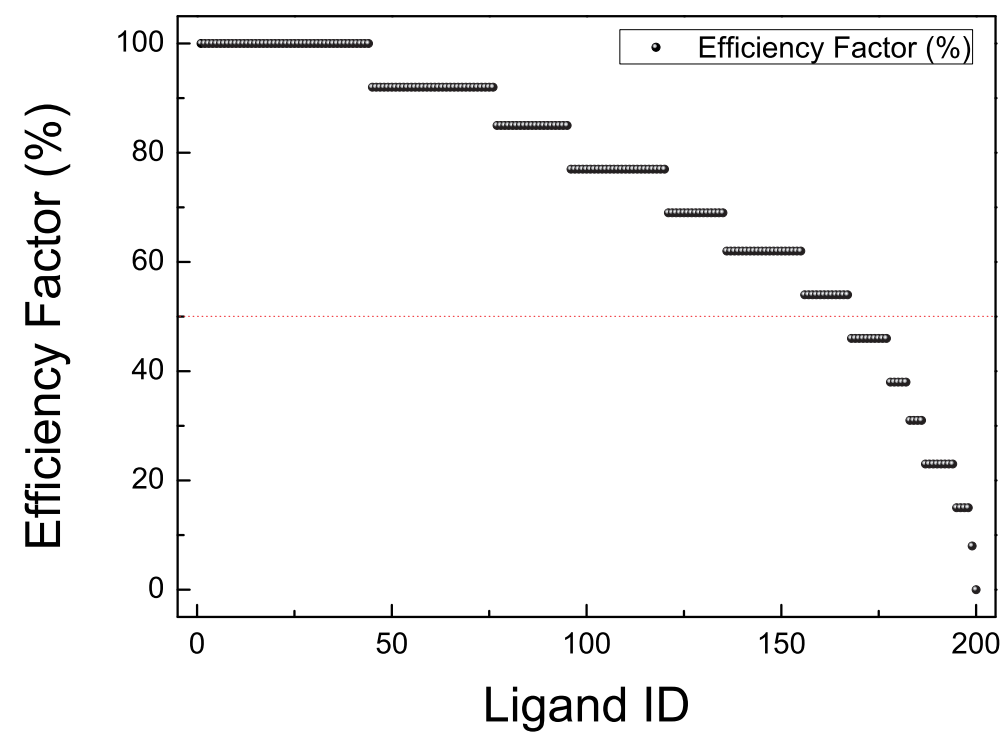

Figure 2.4: The efficient factors of the ligands in discriminating the true targets from the non-targets predicted by the ensemble docking algorithm. 

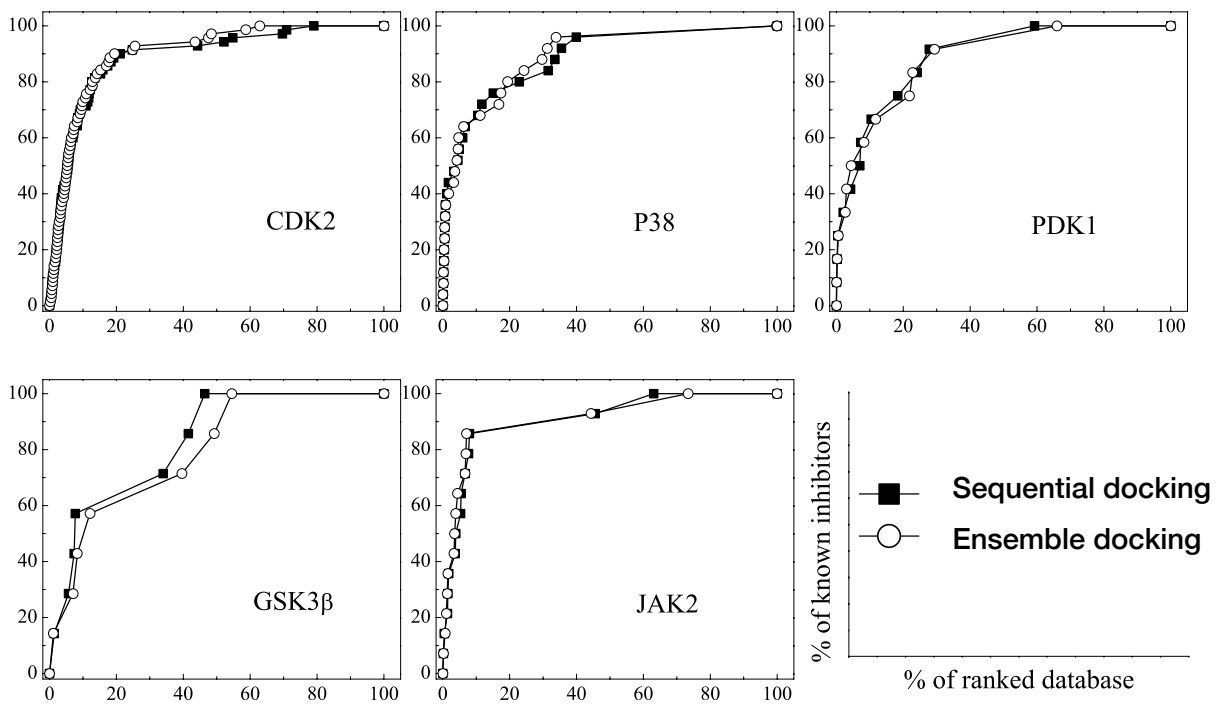

Figure 2.5: Comparisons of the enrichments between ensemble docking and standard sequential docking on five typical target proteins. The legend applies to all the panels. 


\subsection{Discussion and Conclusion}

Simultaneously docking against multiple target proteins is essential because compounds often inhibit multiple proteins of the same family which results in a challenging issue of ligand specificity. ${ }^{16-18,46-50}$ Our present ensemble docking algorithm addresses this issue by simultaneously docking against multiple targets. The virtual screeing test on 14 protein kinases demonstrates that our ensemble docking algorithm is comparable of simultaneously addressing multiple target proteins, and achieved comparable performances to the standard single-target docking method without a significant increase in the computational time.

There is a similar "in situ cross-docking" approach has been presented and tested on several ensembles of up to six targets by Sotriffer and Dramburg, in which the ligands can be simultaneously docked into one united grid consisting of all the binding sites of multiple targets. ${ }^{18}$ The work closely resembles our algorithm, however, there are major differences. In the approach by Sotriffer and Dramburg, ${ }^{18}$ the binding sites of multiple tartet proteins are separate from each other in the united grid. Therefore, if a ligand has fairly favorable scores against more than one target, the convergence toward to the native, global optimum will be slow. This may limit the applicability of their approach for the protein ensemble of very similar targets or different conformations of the same protein. ${ }^{18}$ Moreover, because the grids from different targets are joined one by one, the united grid will boast a much larger volume than the grid of single target. Therefore, the searching efficiency for a ligand binding mode will be considerably lower as a result of the larger docking available docking space.

Unlike the "in situ cross-docking" approach, our ensemble docking algorithm 
aligns the binding sites of multiple target proteins so that the united grid has almost the same volume as that of single-target docking. Accordingly, our ensemble algorithm is computationally efficient with a run time comparable to that of singletarget docking. The ensemble algorithm can also be applied to different proteins for identifying potential targets of an inhibitor and also to different conformations of the same protein for considering protein flexibility regardless of whether the protein structures are similar or not. In addition, our ensemble docking also assigns ligand binding energy scores for each of the targets so that one can investigate the selectivity of a ligand.

Despite the present success, our ensemble docking algorithm contains limitations, Such as the energy optimization method mentioned in the previous work. ${ }^{21}$ In principle, our ensemble algorithm can simultaneously address unlimited target proteins. However, the energy landscape of the target ensemble will be more rugged with the more proteins, which will, in turn, lower the searching efficiency of an energy optimization method. In this work, we used the same optimization method, SIMPLEX ${ }^{32}$ as the DOCK 4.0 software. ${ }^{20,28}$ Thus, the ligand may sometimes be trapped in a local minimum because SIMPLEX is essentially a local minimization method. ${ }^{32}$ As a result, occasional wrong predictions often originate from the optimization method rather than from the ensemble algorithm itself. One solution is to use a global minimization method, which would not be affected by local minima. However, the global minimization method achieves its efficiency at the cost of consumed time. Therefore, despite the fast and accurate results obtained by the present work, it is necessary to develop an appropriate optimization method with a good balance between speed and efficiency when the number of proteins increased. 
In summary, we have presented an efficient ensemble docking algorithm to simultaneously address multiple target proteins. The algorithm was validated by virtual screening against 14 human protein kinases. The ensemble docking algorithm per-

formed comparably to standard single-target docking without a significant increase in run time. We expect the algorithm to be useful for the investigation of the ligand selectivity in structure-based drug design.

\section{Acknowledgments}

We are thankful to our colleague Xianjin $\mathrm{Xu}$ for his expertise that greatly assisted this study.

We are also greatful to the support to XZ from OpenEye Scientific Software Inc. (Santa Fe, NM), Tripos, Inc. (St. Louis, MO) and MDL Information Systems, Inc. (San Leandro, CA) is gratefully acknowledged. 


\section{Bibliography}

[1] Huang S-Y, Zou X.; Advances and Challenges in Protein-Ligand Docking. Int $J$ Mol Sci. 2010, 11, 3016-3034.

[2] Huang S-Y, Grinter SZ, Zou X.; Scoring Functions and Their Evaluation Methods for Protein-Ligand Docking: Recent Advances and Future Directions. Phys Chem Chem Phys. 2010, 12, 12899-12908.

[3] Grinter SZ, Zou X.; Challenges, Applications, and Recent A dvances of ProteinLigand Docking in Structure-based Drug Design. Molecules 2014, 19, 1015010176 .

[4] Kitchen DB, Decornez H, Furr JR, et al; Docking and Scoring in Virtual Screening for Drug Discovery: Methods and Applications. Nat Rev Drug Discovery. 2004, 3, 935-949.

[5] Brooijmans, N.; Kuntz, I. D. Molecular recognition and docking algorithms. Annu. Rev. Biophys. Biomol. Struct. 2003, 32, 335-373.

[6] Meng X.-Y., Zhang H.-X., Mezei M., Cui M. Molecular Docking: A powerful 
approach for structure-based drug discovery. Current Computer-Aided Drug Design. 2011, 7, 146-157.

[7] Santiago, Daniel N., Pevzner Y.,Durand A.A., Virtual target screening: validation using kinase inhibitors. Journal of chemical information and modeling. 2012, $52,2192-2203$.

[8] Zahler S, Tietze S, Totzke F, Kubbutat M, Meijer L, Vollmar AM, Apostolakis J. Inverse in silico screening for identification of kinase inhibitor targets. Chemistry \& biology. 2007, 14, 1207-1214.

[9] Cohen, P. Protein kinases - the major drug targets of the twenty-first century? Nat. Rev. Drug Discov. 2002, 1, 309-315.

[10] Cherry, M.; Williams, D. H. Recent kinase and kinase inhibitor X-ray structures: Mechanisms of inhibition and selectivity insights. Curr. Med. Chem. 2004, 11, 663-673.

[11] Lahiry Piya, Orkamani Ali, Schork Nicholas J., Hegele Robert A., Kinase mutations in human disease: interpreting genotype-phenotype relationships. Nat Rev Genet. 2010, 11, 1471-0056.

[12] Cohen P, Alessi DR. Kinase Drug Discovery Whats Next in the Field? ACS chemical biology. 2013, 8, 96-104.

[13] Macchiarulo A., Nobeli I., Thornton J. M. (2004). Ligand selectivity and competition between enzymes in silico. Nat. Biotechnol. 2004, 22, 1039-1045.

[14] Seifert MHJ, Kraus J, Kramer B. Virtual high-throughput screening of molecular databases. Curr Opin Drug Discov Devel. 2007, 10, 298-307 
[15] Slynko I, Scharfe M, Rumpf T, Eib J, Metzger E, Schule R, Jung M, Sippl W. Virtual screening of PRK1 inhibitors: ensemble docking, rescoring using binding free energy calculation and QSAR model development. Journal of chemical information and modeling. 2014, 54, 138-50.

[16] Lamb, M. L.; Burdick, K. W.; Toba, S.; Young, M. M.; Skillman, K. G.; Zou, X. Q.; Arnold, J. R.; Kuntz, I. D. Design, docking, and evaluation of multiple libraries against multiple targets. Proteins 2001, 42, 296-318.

[17] Rockey, W. M.; Elcock, A. H. Progress toward virtual screening for drug side effects. Proteins 2002, 48, 664-671.

[18] Sotriffer, C. A.; Dramburg, I. "In situ cross-docking" to simultaneously address multiple targets. J. Med. Chem. 2005, 48, 3122-3125.

[19] The Pharmacological Basis of Therapeutics, 10th ed.; Hardman, J. G., Limbird, L. E., Gilman, A. G., Eds.; McGraw-Hill: New York, 2001.

[20] Kuntz, I. D.; Blaney, J. M.; Oatley, S. J.; Langridge, R.; Ferrin, T. E. A geometric approach to macromolecule-ligand interactions. J. Mol. Biol. 1982, 161, 269-288.

[21] Huang S-Y, Zou X. Ensemble docking of multiple protein structures: Considering protein structural variations in molecular docking. Proteins. 2007, 66, 399-421.

[22] Huang S-Y, Zou X. Efficient molecular docking of NMR structures: Application to HIV-1 protease. Protein Sci. 2007, 16, 43-51.

[23] Huang S-Y, Zou X. An iterative knowledge-based scoring function to predict protein-ligand interactions: I. Derivation of interaction potentials. J Comput Chem. 2006, 27, 1865-1875. 
[24] Huang S-Y, Zou X. An iterative knowledge-based scoring function to predict protein-ligand interactions: II. Validation of the scoring function.J Comput Chem 2006, 27, 1876-1882.

[25] Berman, H. M.; Westbrook, J.; Feng, Z.; Gilliland, G.; Bhat, T. N.; Weissig, H. Shindyalov, I. N.; Bourne, P. E. The Protein Data Bank. Nucleic Acids Res. 2000, 28, 235-242.

[26] Hawkins et al., Conformer Generation with OMEGA: Algorithm and Validation Using High Quality Structures from the Protein Databank and the Cambridge Structural Database J. Chem. Inf. Model. 2010, 50, 572-584.

[27] Hawkins PC, Nicholls A. Conformer generation with OMEGA: learning from the data set and the analysis of failures. Journal of chemical information and modeling. 2012, 52, 2919-2936.

[28] Ewing, T. J. A.; Makino, S.; Skillman, A. G.; Kuntz, I. D. DOCK 4.0: Search strategies for automated molecular docking of flexible molecule database. J. Comput. Aided Mol. Des. 2001, 15, 411-428.

[29] Notredame, C.; Higgins, D. G.; Heringa, J. T-Coffee: A novel method for fast and accurate multiple sequence alignment. J. Mol. Biol. 2000, 302, 205-217.

[30] Ewing, T. J. A.; Kuntz, I. D. Critical evaluation of search algorithms for automated molecular docking and database screening. J. Comput. Chem. 1997, 18, 1175-1189.

[31] Meng, E. C.; Shoichet, B. K.; Kuntz, I. D. Automated docking with grid-based 
energy approach to macromolecule-ligand interactions. J. Comput. Chem. 1992, $13,505-524$.

[32] Nelder, J. A.; Mead, R. A simplex method for function minimization. Computer J. 1965, 7, 308-313.

[33] Liu Z., Li Y., Han L. et al. PDB-wide collection of binding data: current status of the PDBbind databaseBioinformatics 2015, 31, 405-412.

[34] Wang, R. X.; Fang, X. L.; Lu, Y. P.; et al. The PDBbind database: Collection of binding affinities for protein-ligand complexes with known three-dimensional structures. J. Med. Chem. 2004, 47, 2977-2980.

[35] Shatsky, M.; Nussinov, R.; Wolfson, H. J. A method for simultaneous alignment of multiple protein structures. Proteins 2004, 56, 143-56.

[36] Rusinko, III A., Sheridan, R. P., Nilakantan, R. et al.; Using CONCORD to construct a large database of three-dimensional coordinates from connection tables. J.Chem. Inf. Comput. Sci. 1989, 29, 251-255.

[37] Cole, J.C., Murray, C.W., Nissink, J.W.M., Taylor, R. D., and Taylor, R. Comparing protein-ligand docking programs is difficult. Proteins 2005, 60, 325-332.

[38] Haupt VJ, Daminelli S, Schroeder M. Drug promiscuity in PDB: protein binding site similarity is key. PLoS one. 2013, 21, e65894.

[39] Tanramluk D, Schreyer A, Pitt WR, Blundell TL. On the origins of enzyme inhibitor selectivity and promiscuity: a case study of protein kinase binding to staurosporine. Chemical biology \& drug design. 2009, 74, 16-24. 
[40] Kantarjian, Hagop, et al. Hematologic and cytogenetic responses to imatinib mesylate in chronic myelogenous leukemia. New England Journal of Medicine 2002, 346, 645-652.

[41] Demetri, GD., et al. Efficacy and safety of imatinib mesylate in advanced gastrointestinal stromal tumors. New England Journal of Medicine 2002, 347, 472480.

[42] Lin, Yen-Lin, et al. Explaining why Gleevec is a specific and potent inhibitor of Abl kinase. Proceedings of the National Academy of Sciences 2013, 110,16641669.

[43] Lin YL, Roux B. Computational analysis of the binding specificity of Gleevec to Abl, c-Kit, Lck, and c-Src tyrosine kinases. Journal of the American Chemical Society. 2013 135, 14741-53.

[44] Lovera, Silvia, et al. The different flexibility of c-Src and c-Abl kinases regulates the accessibility of a druggable inactive conformation. Journal of the American Chemical Society. 2012, 134, 2496-2499.

[45] Agafonov RV, Wilson C, Otten R, Buosi V, Kern D. Energetic dissection of Gleevec's selectivity toward human tyrosine kinases. Nature structural \& molecular biology. $201421,848-53$.

[46] Bowman AL, Lerner MG, Carlson HA. Protein flexibility and species specificity in structure-based drug discovery: Dihydrofolate reductase as a test system. $J$ Am Chem Soc. 2007, 129, 3634-3640. 
[47] Chen YZ, Zhi DG. Ligand-protein inverse docking and its potential use in the computer search of protein targets of a small molecule. Proteins. 2001, 43, 217226.

[48] Paul N, Kellenberger E, Bret G, Muller P, Rognan D. Recovering the true targets of specific ligands by virtual screening of the Protein Data Bank. Proteins. 2004, 54, 671-680.

[49] Gilson MK. Sensitivity analysis and charge-optimization for flexible ligands: Applicability to lead optimization. J Chem Theory Comput. 2006, 2, 259-270.

[50] Rockey WM, Elcock AH. Rapid computational identification of the targets of protein kinase inhibitors. $J$ Med Chem. 2005, 48, 4138-4152. 


\title{
Chapter 3
}

\section{EDockMS: An Efficient Docking Platform for Multiple Target Screening}

\begin{abstract}
Predicting the most likely protein target(s) for a given ligand from thousands of protein candidates is challenging yet important for finding novel targets to combat diseases such as cancer and bacterial infections. Recently, we have built a web server for Efficient Docking-based Multiple target Screening (EDockMS). EDockMS is a user-friendly ligand-protein docking server for predicting potential therapeutic targets for a query compound of interest. We have also constructed a protein database of 537 targets comprising 3268 protein-ligand complex structures with 3349 binding pockets, providing each target with searchable disease-related information. Our achieved success rates and computational efficiency showed that the server is capable of efficiently predicting the protein target(s), their structures complexed with the
\end{abstract}


query ligand, and searchable disease information about the targets. EDockMS has potentially valuable application for therapeutic intervention.

\subsection{Introduction}

Drug molecules often produce not only valuable therapeutic effects but also side effects (i.e. off-target effects) due to their interactions with multiple targets. Although off-target effects are predominantly undesirable, some of them can have potential applications in the treatment of complex diseases such as cancers. ${ }^{1}$ It is now well-known that the clinical effects of a drug molecule are often the result of its interactions with multiple targets rather than with one target, which has led to a shift in the focus of the multi-target paradigm in both experimental and computational approaches. ${ }^{2,3}$ For example, in the emerging field of polypharmacology, the off-target binding property of an FDA-approved drug is utilized for drug repositioning, which lowers the

development costs and reduces the risks of reusing this drug on new purposes. ${ }^{4-6}$ Consequently, it is highly desired to develop methods for discovering multiple targets.

Docking-based Inverse Virtual Screening (IVS) utilizes a molecular docking algorithm to virtually screen large libraries of protein targets to discover possible side effects and toxicities of new drugs or drug candidates. ${ }^{7,8}$ In contrast to the costly and time-consuming biological experiments, IVS allows for a more efficient framework in identifying potential receptor targets for a compound. The current major challenge in computational target prediction lies in the accuracy and efficiency of the scoring functions in the docking-based methods, which need to be substantially improved 
Recently, we developed a web server, referred to as EDockMS, based on the latest version of our MDock package ((http://zoulab.dalton.missouri.edu/mdock.htm) for efficient multiple target screening. The web server uses a statistical potential-based scoring function, ITScore, ${ }^{9,10}$ which was derived based on an statistical mechanicsbased iterative method. ITScore is computationally efficient for large-scale docking. 7,11,12 Moreover, we constructed a protein target database, referred to as MDTD, as part of the EDockMS server. Specifically, we built MDTD based on two databases, sc-PDB ${ }^{13,14}$ and Therapeutic Target Database (TTD). ${ }^{15}$ The sc-PDB database provides structure files (e.g., separated protein and ligand files and the binding site information) suitable for docking-based computational drug design, and TTD allows therapeutic information to be trackable. However, there is no connection between these two databases. MDTD integrates these two databases, resulting in a comprehensive and accurate database which contains both the structural and disease-relevant information for each protein target.

\subsection{Implementation}

The server construction includes three major components: interface, MDTD preparation, and docking/analysis.

\section{Interface}

The front-end web interface is written in HTML and python. The server hardware consists of shared resources with 24 Intel Xeon cores [Intel(R) Xeon(R) CPU E5-2650 v3@2.30GHz]. 


\section{MDTD preparation}

We extracted PDB $\operatorname{codes}^{16}$ using the UniProt IDs ${ }^{17}$ of the targets annotated in TTD, then mapped the PDB codes to those in sc-PDB so that MDTD are finalized with the target structures and disease-relevant details. In the current version, we included 3268 protein-ligand complexes (with 3349 distinct binding sites) associated with 537 target entries which can be tracked with annotated TTD IDs for biochemical information (see Supplementary material).

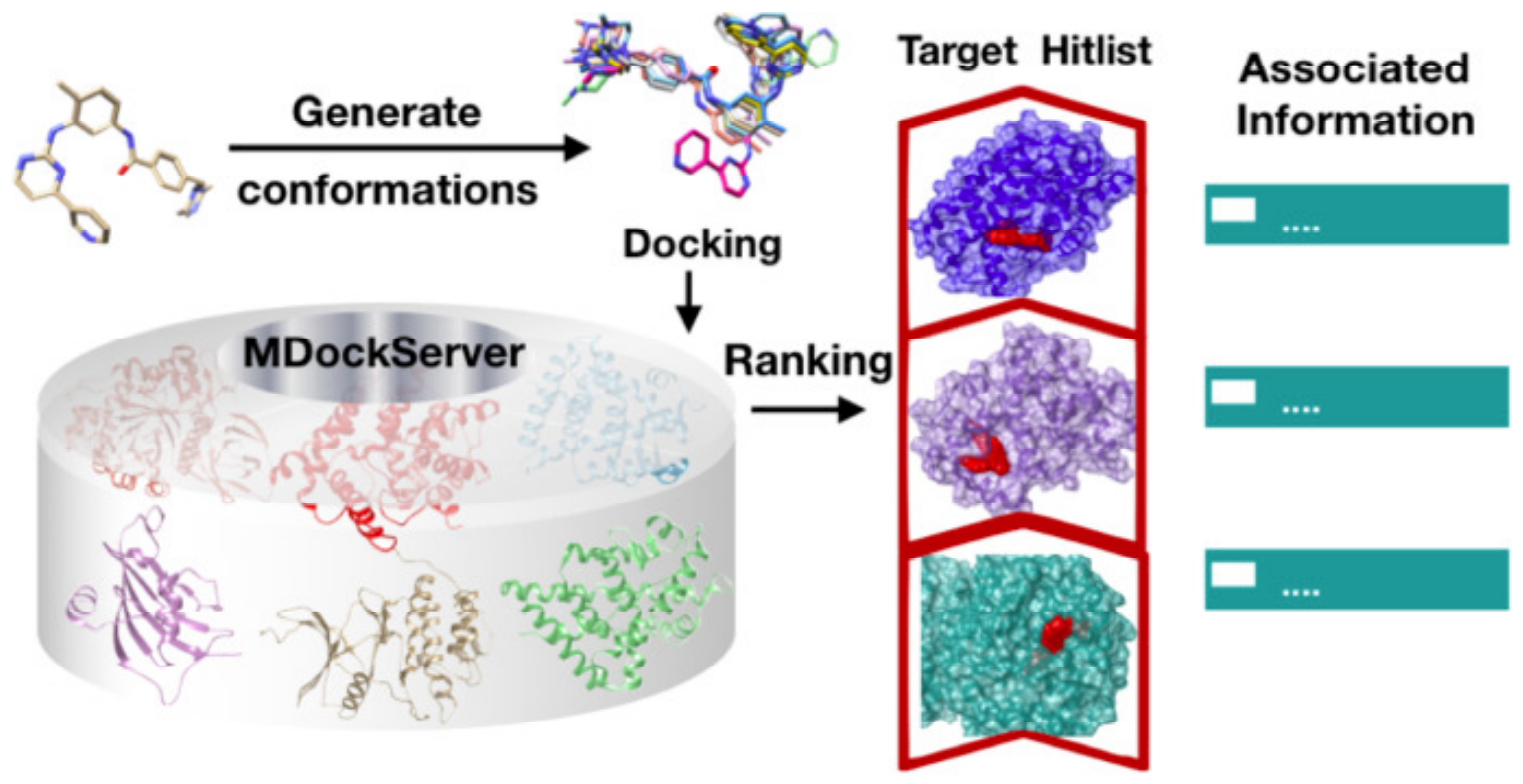

Figure 3.1: Schematic representation that highlights the back-end procedures in EDockMS.

\section{Docking and results analyzation}

EDockMS uses a back-end docking protocol MDock for IVS, via a ligand-protein in-

verse docking strategy. ${ }^{7,8,12}$ Figure 3.1 illustrates the operation of EDockMS. Specif- 
ically, for each submitted query ligand, we use OMEGA (Version 2.5.1. OpenEye Scientific Software, Santa Fe, NM. http://www.eyesopen.com.) to generate conformations for flexible-ligand docking with the default parameter for maxconfs (i.e., 200). ${ }^{18}$ Each conformer will be docked onto all the protein structures in MDTD using our in-house docking protocol MDock. MDock then ranks the generated proteinligand structures with its scoring function, ITScore, keeping only the structure with the best (i.e., most negative) score for each target. The output of each job includes a list of the top 50 protein targets (with disease-related information), and the binding modes for the top 10 targets.

\subsection{Discussion and perspectives}

The docking approach implemented in EDockMS was applied to several studies. ${ }^{7,11,12}$ Importantly, oxidosqualene cyclase was identified as the targets of PRIMA-1 in our previous work. ${ }^{7}$ We further analyzed the performance of EDockMS using an IVS benchmark. ${ }^{19}$ For the 72 drugs in the benchmark, we removed the drugs whose protein targets were absent in the MDTD database. For the remaining drugs with similar structures, we selected the first one in each cluster listed in Table 3 of ref. 19. The final set contained 47 drug ligands (see Supplementary Information). The results showed that EDockMS was able to predict the first true target in 38 out of 47 cases (81\%) among the top $10 \%$ of the dataset consisting of 537 targets. Moreover, the novelty of EDockMS is summarized as follows.

First, EDockMS is user-friendly. Many docking algorithms require users to set up the parameters before docking, which is tedious especially for novices. For job 
submission in EDockMS, only a mol2 file of a ligand structure is required. Once a job is submitted successfully, the status can be checked from the Queue page. Meanwhile, a user has the option to provide an e-mail address, which can be used for notifications when jobs are completed. The results are compressed in a tar file, which can be downloaded directly.

Second, EDockMS is highly efficient, which is a significant feature because dockingbased IVS is computationally costly. In our server, typical runs take 1-4 hours. For example, running IVS for progesterone takes about one hour.

Last, the existing docking web-servers do not provide disease-associated information. With the construction of MDTD, EDockMS provides trackable disease-relevant information for the predicted protein targets, a valuable feature for the further lead optimization.

In conclusion, we have presented the EDockMS server for docking-based IVS with disease-relevant information. The predictions of the top 50 protein targets and the top 10 binding modes are provided via a user-friendly web interface. The benchmarking evaluation results have shown that EDockMS can rapidly identify targets for a query ligand with a high success rate. Future developments include 1) adding the ensemble docking mode to account for protein flexibility, and 2) further classifying the MDTD database for users to focus on a subset of protein targets of interest.

\subsection{ACKNOWLEDGEMENT}

This work is supported by the NSF CAREER Award DBI-0953839 and the NIH R01GM109980 (X.Z.). The computations are performed on the high-performance 
computing infrastructure supported by NSF CNS-1429294 (principal investigator, Chi-Ren Shyu) and the HPC resources supported by the University of Missouri Bioinformatics Consortium (UMBC). 


\section{Bibliography}

[1] Saginc, G.; Voellmy, F.; Linding, R. Nature chemical biology 2017, 13, 1204.

[2] Hopkins, A. L. Nature chemical biology 2008, 4, 682.

[3] Medina-Franco, J. L.; Giulianotti, M. A.; Welmaker, G. S.; Houghten, R. A. Drug discovery today 2013, 18, 495-501.

[4] Reddy, A. S.; Zhang, S. Expert review of clinical pharmacology 2013, 6, 41-47.

[5] Martorana, A.; Perricone, U.; Lauria, A. Current topics in medicinal chemistry 2016, 16, 2088-2106.

[6] Nagaraj, A. B.; Wang, Q. Q.; Joseph, P.; Zheng, C.; Chen, Y.; Kovalenko, O.; Singh, S.; Armstrong, A.; Resnick, K.; Zanotti, K.; Waggoner, S.; Xu, R.; DiFeo, A. Oncogene 2017, 37, 403-414.

[7] Grinter, S. Z.; Liang, Y.; Huang, S.-Y.; Hyder, S. M.; Zou, X. Journal of Molecular Graphics and Modelling 2011, 29, 795-799.

[8] Chen, Y.; Ung, C. Journal of Molecular Graphics and Modelling 2001, 20, 199218. 
[9] Huang, S.-Y.; Zou, X. Journal of Computational Chemistry 2006, 27, 1866-1875.

[10] Huang, S.-Y.; Zou, X. Journal of computational chemistry 2006, 27, 1876-1882.

[11] Grinter, S. Z.; Yan, C.; Huang, S.-Y.; Jiang, L.; Zou, X. Journal of Chemical Information and Modeling 2013, 53, 1905-1914.

[12] Yan, C.; Zou, X. Computer-Aided Drug Discovery; Springer, 2015; pp 153-166.

[13] Meslamani, J.; Rognan, D.; Kellenberger, E. Bioinformatics 2011, 27, 13241326.

[14] Desaphy, J.; Bret, G.; Rognan, D.; Kellenberger, E. Nucleic acids research 2014, 43, D399-D404.

[15] Li, Y. H. et al. Nucleic acids research 2017, 46, D1121-D1127.

[16] Berman, H. M. Nucleic Acids Research 2000, 28, 235-242.

[17] TheUniProtConsortium, Nucleic Acids Research 2016, 45, D158-D169.

[18] Hawkins, P. C. D.; Skillman, A. G.; Warren, G. L.; Ellingson, B. A.; Stahl, M. T. Journal of Chemical Information and Modeling 2010, 50, 572-584.

[19] Schomburg, K. T.; Bietz, S.; Briem, H.; Henzler, A. M.; Urbaczek, S.; Rarey, M. Journal of chemical information and modeling 2014, 54, 1676-1686. 


\title{
Chapter 4
}

\section{Predicting Protein Ligand Binding Modes for CELPP and GC3: Workflows and Insight}

The work in this chapter has been published in Methods in J Comput Aided Mol Des. ${ }^{1}$

\begin{abstract}
Drug Design Data Resource (D3R) continues to release valuable benchmarking datasets to promote improvement and development of computational methods for new drug discovery. We have developed several methods for protein-ligand binding mode prediction during the participation in the D3R challenges. In the present study, these methods were integrated, automated, and systematically tested using the large-scale data from Continuous Evaluation of Ligand Pose Prediction (CELPP) and a subset of Grand challenge 3 (GC3). The results show that current molecular docking methods
\end{abstract}

\footnotetext{
${ }^{1} \mathrm{X} . \mathrm{Xu}^{\dagger}$, Z. $\mathrm{Ma}^{\dagger}$, R. Duan ${ }^{\dagger}, \mathrm{X}$. Zou. Predicting proteinligand binding modes for CELPP and GC3: workflows and insight, Scoring and in Silico Screening. J Comput Aided Mol Des, 2019 ( $^{\dagger}$ Equally contributed).
} 
benefit from the increasing number of protein-ligand complex structures deposited in Protein Data Bank. Using an appropriate protein structure for docking significantly improves the success rate of the binding mode prediction. The results of our templatebased method and docking method are compared and discussed. Our future direction include the combination of these two methods for binding mode prediction.

\subsection{Introduction}

In silico methods for protein-ligand complex structure prediction (e.g. molecular docking) play an increasingly important role in modern drug discovery and development. ${ }^{1-3}$ Although great successes have been achieved, current methods are facing several challenges such as the protein flexibility problem and the scoring function problem. ${ }^{4,5}$ To promote the improvement of the existing methods and the development of new approaches, Drug Design Data Resource (D3R,(https ://www .drugdesigndata. org), starting from 2015) continues to release valuable benchmarking datasets containing experimentally determined binding structures and affinity data. ${ }^{6-8}$ Recently, the D3R Team has developed Continuous Evaluation of Ligand Pose Prediction (CELPP), an automated workflow to process and evaluate the challenge of proteinligand binding mode prediction. CELPP is held weekly, in which the targets are

prepared based on pre-released data from the Protein Data Bank (PDB) ${ }^{9}$ (including the ligands and the sequence of their target proteins). In addition to CELPP, D3R organizes one or two rounds of Grand Challenge (GC) every year for both binding mode and binding affinity predictions. In the latest Grand Challenge 3, D3R released a subset of 24 compounds targeting the human Cathepsin S (CatS) for binding mode 
prediction.

Through the participation in the D3R and its previous predecessor, Community Structure-Activity Resource (CSAR, (http://www.csardock.org), 2010-2014), ${ }^{10-13}$ we developed several novel strategies for predicting protein-ligand complex structures. ${ }^{14-20}$ During the CSAR challenges, we improved our in-house scoring function, ITScore2, an efficient statistical potential-based scoring function derived by using a statistical mechanics-based iterative method based on a training set of experimentally determined proteinligand complex structures [15]. During the GC 2015, we improved both binding mode and binding affinity predictions of molecular docking by using the protein structures that contain the co-bound ligands sharing high molecular similarities with the query ligands. ${ }^{14}$ A system-specific scoring function was also introduced in this round of GC. Then, in GC2, we developed a template-based approach using known protein-ligand complex structures as templates. ${ }^{15}$ These methods use known protein-ligand complex structures in different ways to improve the performance of binding mode prediction, and benefit from the increasing number of experimentally determined protein-ligand complex structures deposited in the PDB.

In this study, we thoroughly analyzed the binding mode prediction results from our recent participation in the D3R challenges, CELPP and GC3. For CELPP, we predicted more than one thousand targets in 42 CELPP weeks (from week22_2017 to week11_2018). For each target, a molecular docking approach was performed using up to 6 protein structures. Five of these protein structures were provided by CELPP. The 6th protein structure was selected from all the known experimental structures of the same protein by using a 3D molecular similarity calculation method. The docking results showed that using a proper protein structure (e.g., containing a co-bound 
ligand that shares a high molecular similarity with the query ligand) significantly improved the performance of binding mode prediction for the docking method. In addition to molecular docking, a template-based method was also used and the results were compared with the docking results. The analysis showed that the performance of the template-based method highly depends on the molecular similarities between the co-bound ligands in the templates and the query ligands. Similar results were observed for the GC3 targets (CatS subset). Through systematic validation on the large-scale data from D3R (CELPP and GC), we continue to improve our protein-ligand complex structure prediction methods (both docking-based and template-based), which would become a useful, user-friendly tool for computer-aided drug design (CADD) and for biologists' use.

\subsection{Materials and Methods}

\subsubsection{The query protein-ligand complexes}

\section{The CELPP targets}

CELPP releases 20-100 upcoming protein-ligand complex targets every Saturday (12:01 am), and the participants are required to submit predicted binding modes by the following Tuesday $(2: 59 \mathrm{pm})$. Experimental structures will be released in the Protein Data Bank on Wednesday (i.e., the day after the submission deadline). For each target, one query ligand and up to five candidate protein structures are provided. These five protein structures are named as LMCSS, SMCSS, hiResHolo, hiResApo, and hiTanimoto. LMCSS stands for the structure that contains the ligand with the 
largest $\underline{\text { maximum }}$ common substructure to the query ligand. In contrast, SMCSS represents the structure that contains the ligand with the smallest maximum common substructure to the query ligand. hiResHolo denotes the highest-resolution, ligandbound protein structure, and hiResApo marks the highest-resolution, apo protein structure. Similar to LMCSS, hiTanimoto corresponds to the structure that contains a ligand sharing the highest similarity (calculated by Tanimoto coefficient) with the query ligand. In addition, the participants are allowed to submit predictions based on other protein structures. For each protein structure, participants are asked to submit only one predicted binding mode. More details regarding the CELPP targets are available at the website https://drugdesigndata.org/about/celpp.

In this study, we analyzed our predictions based on the 1472 targets that we submitted from week22_2017 to week11_2018. A total of 1636 targets were released during these 42 weeks. Failed submissions were mainly due to the uncommon atoms (such as As) in the query ligands. In addition, for 295 cases out of these 1636 targets, their LMCSS or hiTanimoto protein structures contain co-bound ligands that were identical to the query ligands. These 295 targets were therefore discarded in the analysis of this study.

\section{The GC3 targets}

Grand Challenge 3 released a subset for proteinligand binding mode prediction. This subset contains 24 query ligands targeting the human Cathepsin S (CatS). The organizers provided the SMILES strings of the query ligands, and the participants were free to use any structures of CatS. For each query ligand, the participants were allowed to submit up to 5 predicted binding modes. 


\subsubsection{Overview of our binding mode prediction methods}

\section{For CELPP}

For the protein structures provided by CELPP, we used a docking strategy that was developed during previous D3R challenges. Briefly, a modified version of AutoDock Vina $^{16,21}$ was used to generate up to 500 ligand binding modes on a target protein. Then, these putative binding modes were re-ranked using our in-house scoring function, ITScore2. ${ }^{16}$ Finally, the top binding mode based on each protein structure was submitted for each target.

ITScore2 was a statistical-potential-based scoring function. It was derived using an iterative method that circumvents the reference state problem by improving atompair potentials iteratively through the comparison of the calculated $\left(g_{i j}^{k}(r)\right)$ and the experimental pair distribution functions $\left(g_{i j}^{*}(r)\right)$ until the native complex structures in the training database can be correctly discriminated from decoy structures. A detailed description of the ITScore 2 method is available in the references. ${ }^{16,22,23}$

Moreover, in addition to the predictions based on the protein structures provided by CELPP, we also submitted a prediction based on our own selected protein structure for each target. This protein structure was selected based on our calculated 3D molecular similarity between the co-bound ligand and the query ligand. Namely, the protein structure that contains a co-bound ligand sharing the highest 3D molecular similarity with the query ligand according to our calculation was used for binding mode prediction. 


\section{For GC3}

Two approaches were used in binding mode prediction for GC3. The first approach was the docking approach that was used for CELPP. The protein structure that contains a co-bound ligand sharing the highest 3D molecular similarity with the query ligand according to our calculation was used for docking. The second approach was a template-based method. Briefly, the query ligand was superimposed onto the ligands in the known protein-ligand complexes, and the protein-query ligand complexes were ranked using the scoring function ITScore2. The top 5 models were further refined by the minimization step in molecular dynamics (MD) simulation.

\subsubsection{Protein preparation}

\section{For CELPP}

In addition to the protein structures provided by CELPP, we also selected a protein structure from the PDB for each target, referred to as the user-specified protein struc-

ture. Specifically, the program Protein-Protein BLAST (v2.6.0+) ${ }^{24,25}$ was employed to search protein structures deposited in the PDB using the protein sequence provided by CELPP. The cutoff was set to $90 \%$ for both the sequence identity and the sequence coverage. The apo structures (i.e., the structures without co-bound ligands) were discarded. If there were more than one PDB structure that contain the same ligand, the structure with the lowest $\mathrm{R}$-free value was kept. Then, 3D molecular similarities between the co-bound ligands and the query ligand were calculated by the program SHAFTS. ${ }^{26}$ The PDB structure that contained the ligand sharing the highest similarity with the query ligand was selected for docking. The whole process 
was automatically performed for a given target.

\section{For GC3}

For the Grand Challenge, we manually checked the CatS structures in PDB, and found 12 PDB entries of human CatS containing different co-bound ligands. These PDB entries (2F1G, 2G7Y, 2H7J, 2R9M, 2R9O, 3IEJ, 3KWN, 3MPE, 3MPF, 3N3G, 4P6E, and 4P6G) were used for both docking method and template-based method.

\subsubsection{Ligand preparation and similarity calculation}

Up to 500 3D conformers were generated for each query ligand, using the program OMEGA (version 2.5.1.4, OpenEye Scientific Software, Santa Fe, NM. http: //www . eyesopen. com $)^{27,28}$ based on the SMILES string provided by CELPP or GC3. Only one conformer for each query ligand was necessary for molecular docking, because the ligand was treated as a flexible molecule in docking. The multiple ligand conformers were for the calculation of the 3D molecular similarities between a query ligand and the co-bound ligands in the known proteinligand complex structures, using the program SHAFTS. ${ }^{26}$ The similarity score calculated by SHAFTS consists of a ShapeScore (i.e., shape-densities overlap) and a FeatureScore (i.e., pharmacophore feature fit values); both scores are normalized to a range of $[0,1]$, and the resulting HybridScore (i.e., the sum of ShapeScore and FeatureScore) is scaled to [0, 2], with 0 representing no similarity and 2 corresponding to the same ligand. 


\subsubsection{Binding mode prediction}

\section{The docking approach}

AutoDock Vina ${ }^{21}$ was employed for sampling putative binding modes of a query ligand on a target protein. For the CELPP targets, we used the binding site center provided by CELPP as the center of the search box. The size of the cubic search box depended on the size of the query ligand. Specifically, the edge length of the cubic box was set to $\max [2 * \operatorname{maxL}, 22.5 \AA]$, in which maxL was the maximum length of the query ligand structure in all dimensions. For the GC3 targets, the geometric center of the co-bound ligand was used as the center of the search box. The size of the search box was set to $30 \AA$. For both CELPP targets and GC3 targets, the protein structures were treated as rigid bodies and single bonds of the ligands were considered to be flexible. The exhaustiveness value was increased to 30 (default=8) to ensure exhaustive sampling. The original Vina source code was modified to set the maximum of output modes to 500 (default=20). The output modes were then re-ranked using an in-house scoring function ITScore2. ITScore2 is a knowledge-based atomic pairwise scoring function that was derived based on the known proteinligand complex structures (a refined set of PDBbind 2012 containing 2897 proteinligand complexes $^{29,30}$ ) and the statistical mechanics-based iterative method ${ }^{16,22,23}$. Finally, the top mode was submitted for each CELPP target protein structure, and the top 5 modes were submitted for each GC3 target. 


\section{The template-based approach}

During Grand Challenge 2, we developed a template-based approach for proteinligand complex structure prediction. The details of the approach are described in Reference. ${ }^{15}$ Briefly, molecular similarities of a query ligand with the co-bound ligands in the known complex structures were calculated using the program SHAFTS. Then, the top 5 PDB entries that had the best similarity scores with the query ligand were chosen as the templates. For each template, the query ligand was superimposed onto the ligand using the program SHAFTS. Finally, the protein-query ligand complex was refined using the minimization step of the MD simulation.

A drawback of this template-based approach is that severe clashes/overlaps between protein atoms and ligand atoms were found in the superimposition step (i.e., before minimization) in some cases, particularly in the cases in which only low-quality templates were available. Even MD minimization were unable to remove the clashes. To avoid or reduce these clashes/overlaps, we introduced a re-scoring step after the superimposition step in GC3. Specifically, instead of using the top 5 best PDB entries as the templates, we superimposed each conformer of the query ligand onto all the co-bound ligands in the known complex structures. Then, all the generated

protein-query ligand complex structures were ranked using their scores calculated by the scoring function ITScore2. If the ligand RSMD of the two models was less than $2.0 \AA$, the model with a more negative score was kept. Finally, the top 5 models were refined using the minimization step of the MD simulation. 


\subsection{Results and Discussion}

To evaluate the quality of predicted binding modes, we calculated root-mean-square deviations (RMSDs) between the predicted ligand binding modes and the modes in the released experimental complex structures. Specifically, the protein structures were matched using the MatchMaker tool of UCSF Chimera, ${ }^{31}$ and the RMSDs of the heavy atoms in the ligands were calculated using the maximum common substructure (MCS) functionality of the OEChem Python toolkit (version 2.5.1.4, OpenEye Scientific Software, Santa Fe, NM. http://www. eyesopen.com). ${ }^{27,28}$ The MCS functionality can handle ligand atom renumbering and account for compound symmetries that are often involved in ligand superimposition.

\subsubsection{CELPP}

For each target, CELPP provided up to 5 protein structures, which were named as candidate protein structures. In addition to the predictions based on these candidate protein structures, we also submitted a prediction based on our own selected protein structure (referred to as the user-specified protein structure) for each target. The prediction results for using the CELPP-provided protein structures and the userspecified protein structures are reported as follows.

\section{The CELPP-provided protein structures}

CELPP provided the binding site location for each target. However, for a number of cases, the provided binding site locations were far from those in the released experimental complex structures. Here, we analyzed only the cases in which the binding 
site information was correctly provided. Namely, the targets were discarded if the distance between the geometry centers of the CELPP binding site and the real binding site were larger than $10 \AA$. The number of the targets that were kept for each type of protein structures are reported in Table 4.1.

Table 4.1: The results of binding mode prediction for the CELPP targets

\begin{tabular}{ccccccccc}
\hline & & \multicolumn{3}{c}{ Top 1 } & \multicolumn{3}{c}{ Best in Top 5} & \multicolumn{2}{c}{ Best in All } \\
\cline { 3 - 8 } Protein & $\begin{array}{c}\text { Number } \\
\text { of } \\
\text { Structure }\end{array}$ & $\begin{array}{c}\text { Median } \\
\text { RMSD } \\
\text { Targets }\end{array}$ & $\begin{array}{c}\text { Median } \\
\text { RMSD }\end{array}$ & $\begin{array}{c}\text { Median } \\
(\AA)\end{array}$ & $\begin{array}{c}\text { RMSD } \\
(\AA)\end{array}$ & $\begin{array}{c}\text { Median } \\
\text { RMSD } \\
(\AA)\end{array}$ & $\begin{array}{c}\text { Median } \\
\text { RMSD } \\
(\AA)\end{array}$ & $\begin{array}{c}\text { Median } \\
\text { RMSD } \\
(\AA)\end{array}$ \\
\hline Bound & 969 & $4.3(0.3)$ & $4.9(0.1)$ & $2.3(0.1)$ & $3.3(0.1)$ & $1.2(0.0)$ & $1.5(0.0)$ \\
LMCSS & 969 & $4.6(0.2)$ & $5.1(0.1)$ & $2.6(0.1)$ & $3.5(0.1)$ & $1.5(0.0)$ & $1.9(0.0)$ \\
SMCSS & 969 & $5.7(0.2)$ & $6.0(0.1)$ & $3.5(0.1)$ & $4.2(0.1)$ & $1.9(0.1)$ & $2.4(0.1)$ \\
hiResApo & 608 & $6.4(0.2)$ & $6.5(0.1)$ & $4.4(0.2)$ & $4.8(0.1)$ & $2.2(0.1)$ & $2.8(0.1)$ \\
hiResHolo & 969 & $5.6(0.2)$ & $5.8(0.1)$ & $3.5(0.1)$ & $4.2(0.1)$ & $1.8(0.1)$ & $2.4(0.1)$ \\
hiTanimoto & 969 & $4.9(0.2)$ & $5.2(0.1)$ & $2.6(0.1)$ & $3.5(0.1)$ & $1.5(0.0)$ & $1.9(0.0)$ \\
hiSHAFTS & 875 & $4.7(0.2)$ & $5.3(0.1)$ & $2.7(0.1)$ & $3.6(0.1)$ & $1.6(0.1)$ & $1.9(0.0)$ \\
\hline
\end{tabular}

The first row ("Bound") lists the results of using the bound protein structures for docking. The last row (hiSHAFTS) gives the results from the use of the user-specified protein structures (i.e., the protein structure that contains the ligand with the largest HybridScore to the query ligand) for docking, and the other five rows show the results from the use of the candidate protein structures provided by CELPP. The error of each value was estimated using the bootstrap method in which 1000 replicates were used, and was reported in parentheses. ${ }^{32,33}$ 
Figure 4.1 shows the results of binding mode prediction using different RMSD values as the thresholds to define successful predictions. The vertical dashed black lines correspond to a commonly-used threshold value of 2.0 . In addition to the docking results using CELPP-provided protein structures, we also present the results of docking using bound protein structures (i.e. the protein structures extracted from the experimental complex structures) for reference. In bound dockings, ligand structures were generated from the SMILES strings. Figure 1a shows the results when the top model was considered for each protein structure. Using the threshold of 2.0 as an example, bound docking yielded the highest success rate $30.5 \%$, as expected. The LMCSS protein structures (which contain similar ligands with the query ligands) resulted in a significantly higher success rate (23.2\%) than the SMCSS protein structures (which contain dissimilar ligands with the query ligands) for which the success rate was $13.6 \%$. The highest resolution ligand-bound candidate protein structures (hiResHolo) yielded slightly better docking performance (a success rate of 14.8\%) than SMCSS, but resulted in significantly worse performance than LMCSS. When the hiResApo protein structures (i.e., the highest-resolution unbound protein structures) were used, the success rate was $8.1 \%$, which was lower than all the other cases. Similar to LMCSS, the hiTanimoto protein structures contained similar ligands with the query ligands and achieved comparable performances with LMCSS. The same conclusion can be obtained using different threshold values (see Fig. 4.1). 

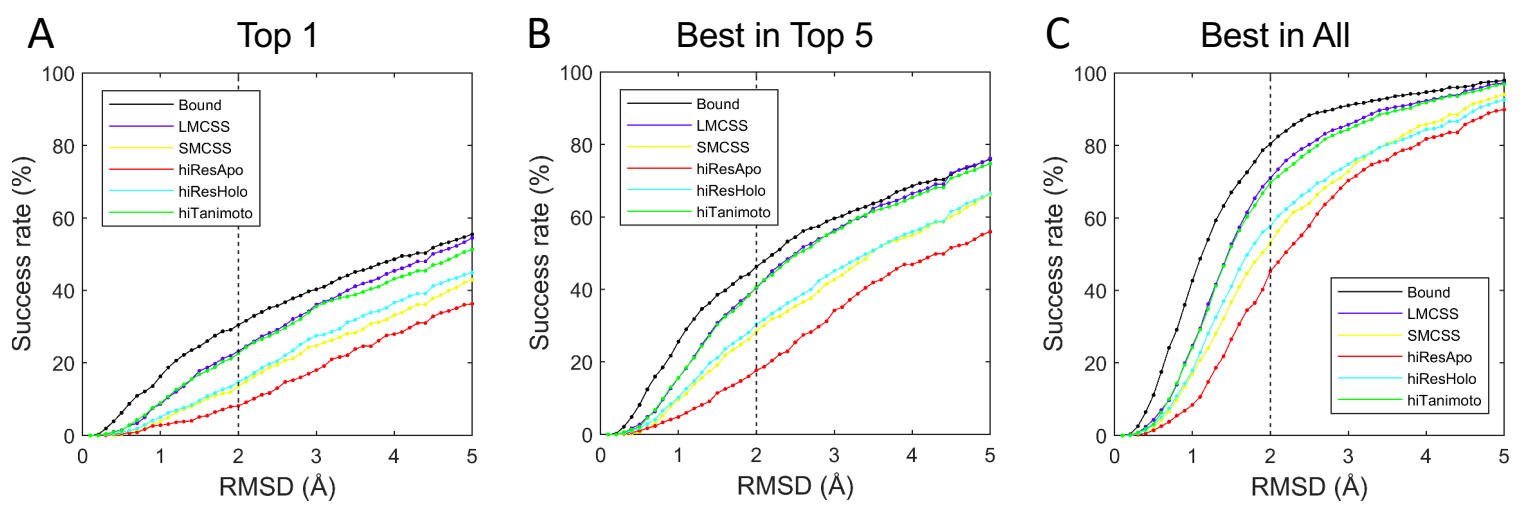

Figure 4.1: The results of binding mode prediction for CELPP using the bound protein structures and the candidate protein structures provided by CELPP for docking. Different RMSD values were used as the thresholds that defined successful predictions. (A) The success rate when only the top predicted model was considered for each protein structure. (B) The success rate when top 5 models were considered for each protein structure. (C) The success rate when all the models were considered for each protein structure.

Figure 4.1B presents the results when top 5 models were considered for each protein structure. Dramatically higher success rates were observed for all types of protein structures, in comparison with those when only the top model was considered. Specifically, when $2.0 \AA$ was used as the threshold, the success rates of dockings with the bound, LMCSS, SMCSS, hiResHolo, hiResApo, and hiTanimoto protein structures increased to $46.3 \%, 40.4 \%, 28.5 \%, 30.4 \%, 17.7 \%$, and $40.6 \%$, respectively. Lower success rates observed in Fig. 4.1a (considering only the top model) were mainly due to the inaccuracy of the current scoring function (ITScore2).

In addition to using the in-house scoring function, ITScore2, we also analyzed the performance of the Vina Score. The results are presented in the Supplementary Materials (Fig. S2). Vina Score achieved similar success rates as ITScore2 for each type of protein structures when the top model was considered. However, ITScore2 
yielded significantly better performance than Vina Score when top 5 models were considered. In addition, similar to what was observed for ITScore2, the bound docking yielded the highest success rate. Dockings with the protein structures containing similar ligands with the query ligands (LMCSS or hiTanimoto) achieved significantly higher success rates than dockings with other types of protein structures (SMCSS, hiResHolo, or hiResApo).

Figure 4.1C shows the sampling results for the use of each type of protein structures for docking. Using $2.0 \AA$ as the threshold, bound docking yielded the highest success rate (80.4\%), similar to the results in Fig. 1a, b. Possible reasons for the failures of sampling in some targets could be the non-exhaustive sampling strategy used in Vina and the ignorance of the effects of crystal waters or co-factors during docking. For the cases using the CELPP-provided protein structures for docking, LMCSS or hiTanimoto protein structures resulted in significantly higher success rates than other CELPP-provided protein structures. Specifically, when $2.0 \AA$ was used as the threshold, the success rates of dockings with the LMCSS, SMCSS, hiResHolo, hiResApo, and hiTanimoto protein structures were $71.0 \%, 53.0 \%, 57.7 \%, 45.3 \%$, and $69.8 \%$, respectively.

In addition to the success rates shown in Fig. 4.1, the median and mean RMSD values for the use of each type of protein structures for docking are reported in Table 4.1. Similar to the results presented in Fig. 4.1, docking with the LMCSS and hiTanimoto protein structures achieved similar performances, and their median/mean RMSD values were significantly lower than dockings with the other three types of CELPP-provided protein structures. For instance, as shown in Columns 3 and 4 , when the top predicted model was considered for each protein structure, the 
LMCSS protein structures resulted in significantly lower median/mean RMSD values $(4.6 \pm 0.2 / 5.1 \pm 0.1 \AA)$ than the SMCSS protein structures $(5.7 \pm 0.2 / 6.0 \pm 0.1 \AA)$. The highest resolution ligand-bound candidate protein structures (hiResHolo) yielded slightly better docking performance $(5.6 \pm 0.2 / 5.8 \pm 0.1 \AA)$ than SMCSS, but resulted in significantly worse performance than LMCSS. When the hiResApo protein structures were used, the median/mean RMSD values were $6.4 \pm 0.2 / 6.5 \pm 0.1 \AA$, which were larger than all the other cases. Similar to LMCSS, the hiTanimoto protein structures, which contained similar ligands with the query ligands, achieved comparable performances with LMCSS. The results based on the Vina Score are also reported in Table S1.

In summary, dockings using the LMCSS or hiTanimoto protein structures achieved significantly better performances than dockings using other CELPP-provided protein structures. Similar conclusions were also obtained in the previous Grand Challenges (GC1 and GC2), in which we found that using protein structures containing a cobound ligand sharing a high molecular similarity with the query ligand would significantly improve the binding mode prediction. ${ }^{14,15}$ The above findings are consistent with the assumption that similar protein conformations, particularly similar conformations of the binding pockets, would be induced by binding of similar ligands, and that similar ligands tend to manifest similar binding modes. Next, we will present the results of prediction based on our own selected protein structures (i.e., user-specified protein structures), which were chosen with a 3D molecular similarity calculation method. 


\section{The user-specified protein structures}

Fig. 4.2A shows the performances using different RMSD values as the thresholds for predictions based on our own selected protein structures (referred to as hiSHAFTS). For each target, docking was performed on the protein structure that contains a co-bound ligand sharing the highest 3D molecular similarity with the query ligand. The molecular similarity was calculated using the program SHAFTS with the default

cutoff of 0.8. Based on the 875 evaluated targets, using $2.0 \AA$ as the threshold for a correct prediction, the success rates were $22.6 \%, 39.0 \%$, and $67.7 \%$ for the top model, the best in top 5 models, and the best in all sampled models, respectively. The median and mean RMSD values of binding mode prediction using hiSHAFTS are also reported in Table 4.1. The performances were similar to the performances using the best candidate protein structures (i.e., LMCSS or hiTanimoto structures provided by CELPP). 

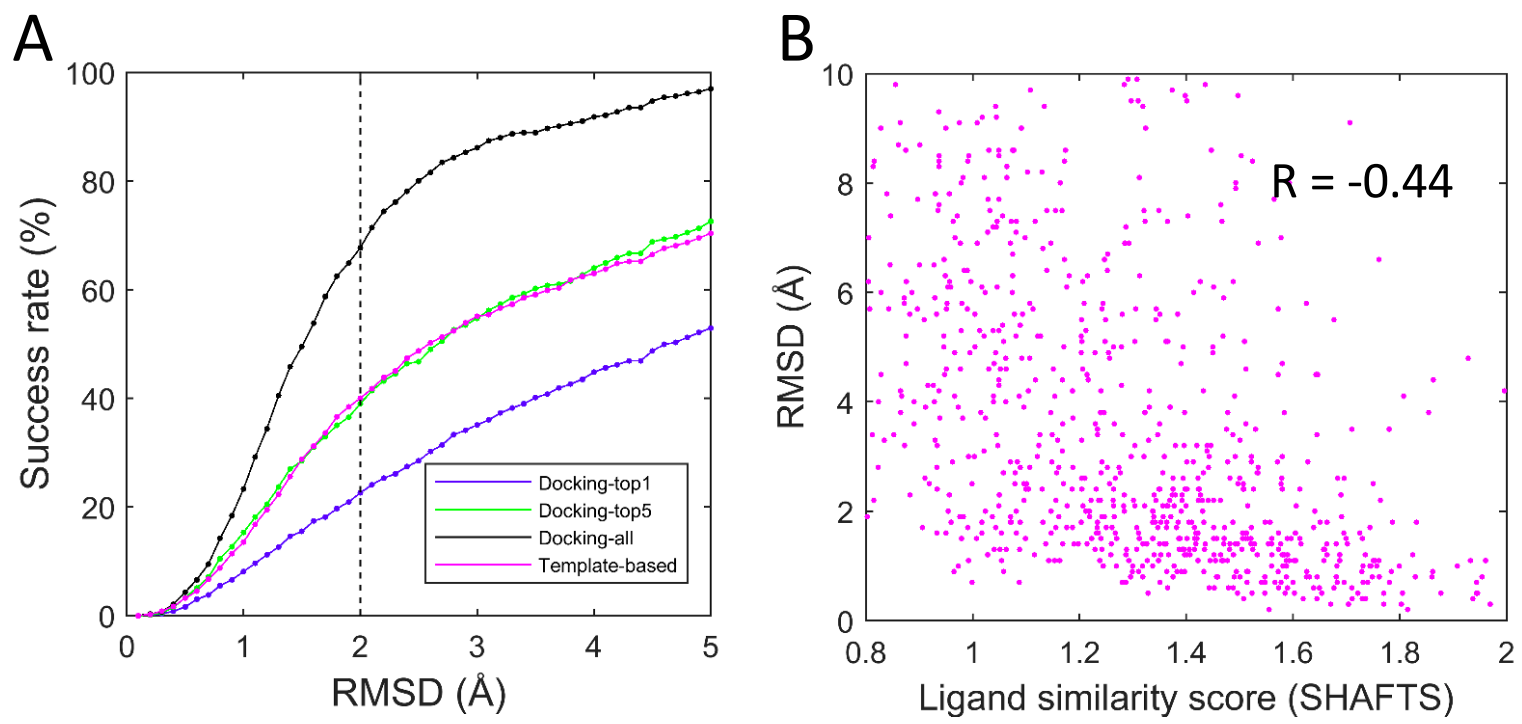

Figure 4.2: A The results of binding mode prediction for CELPP using the userspecified protein structures (i.e. hiSHAFTS structures in this study) for both dockingbased and template-based methods. Different RMSD values were used as the thresholds for the definition of successful predictions. B The correlation between the RMSD values of the binding modes predicted by the template-based method and the corresponding similarity scores between the query ligands and the corresponding template ligands

Figure $4.2 \mathrm{~A}$ also shows the results of the template-based method. Here, the hiSHAFTS complex structure was used as the template for each target. The query ligand was superimposed onto the co-bound ligand in the hiSHAFTS complex structure. It should be noted that the MD-based refinement step for the generated protein-query ligand complexes was not performed in the CELPP study, mainly due to the large number and complexity of the CELPP targets. It is surprising that a simple superimposition of the query ligand onto the template achieved a much better performance than the docking-based method. When $2.0 \AA$ was used as the threshold, the success rate of the template-based method was $40.0 \%$, about twice of the success rate 
achieved by the docking method (considering only the top model).

Furthermore, we analyzed the correlation between the performance of the templatebased method and the quality of the templates used for prediction. Figure 4.2B plots the RMSD values of each target vs the corresponding similarity scores between the query ligands and the corresponding ligands in the templates, showing that targets with higher similarity values tend to have lower $R M S D$ values $(R=0.44)$. The value of 1.2 is the default cutoff of SHAFTS for a distinction of similar and dissimilar ligands. In this study, at least one good-quality template (SHAFTS score1.2) were found for about $63.4 \%$ of the 875 evaluated cases. If only the cases in which SHAFTS score1.2 were considered, the success rate increased to $53.1 \%$ using $2.0 \AA$ as the threshold. The success rate increased to as high as $71.2 \%$ if the SHAFTS score cutoff was set to 1.5 .

Although the template-based method achieved higher success rate than the docking method for binding mode prediction, the performance of the template-based method was highly dependent on the quality of the templates. The method usually failed if no good-quality template (e.g. SHAFTS scorej1.2) was found. Furthermore, sometimes large RMSD values were also observed even if high-quality templates (e.g. SHAFTS score1.5) were (see Fig. 4.2B). The failures suggest the need for the improvement of our current template-based method, in which the query ligands were simply superimposed onto the template ligands and the protein induced-fit effects were ignored. 


\section{GC3}

The template-based method also achieved good performance for the CatS subset, which contains 24 compounds targeting the human Cathepsin S. The median/mean RMSD values are 3.5/4.3 (2.4/3.4) $\AA$ when the top model (top 5 models) was (were) considered for each case. Unfortunately, poor performance was observed with the docking method. The median/mean RMSD values were as high as 8.3/9.9 (5.9/6.5) $\AA$ when the top model (top 5 models) was (were) considered for each case. The predictions did not improve when the bound protein structures were used for docking. Analyzing the targets in this subset showed that the targets contain large-size flexible compounds and large-size binding pocket and that relatively few interactions were found in the released crystal structures (see Fig. 4.3 for an example), indicating that entropy may play an important role in binding for this subset. The failure of the current docking method is probably due to the inaccuracy of the scoring function for the entropy calculation for large-size ligand molecules. 


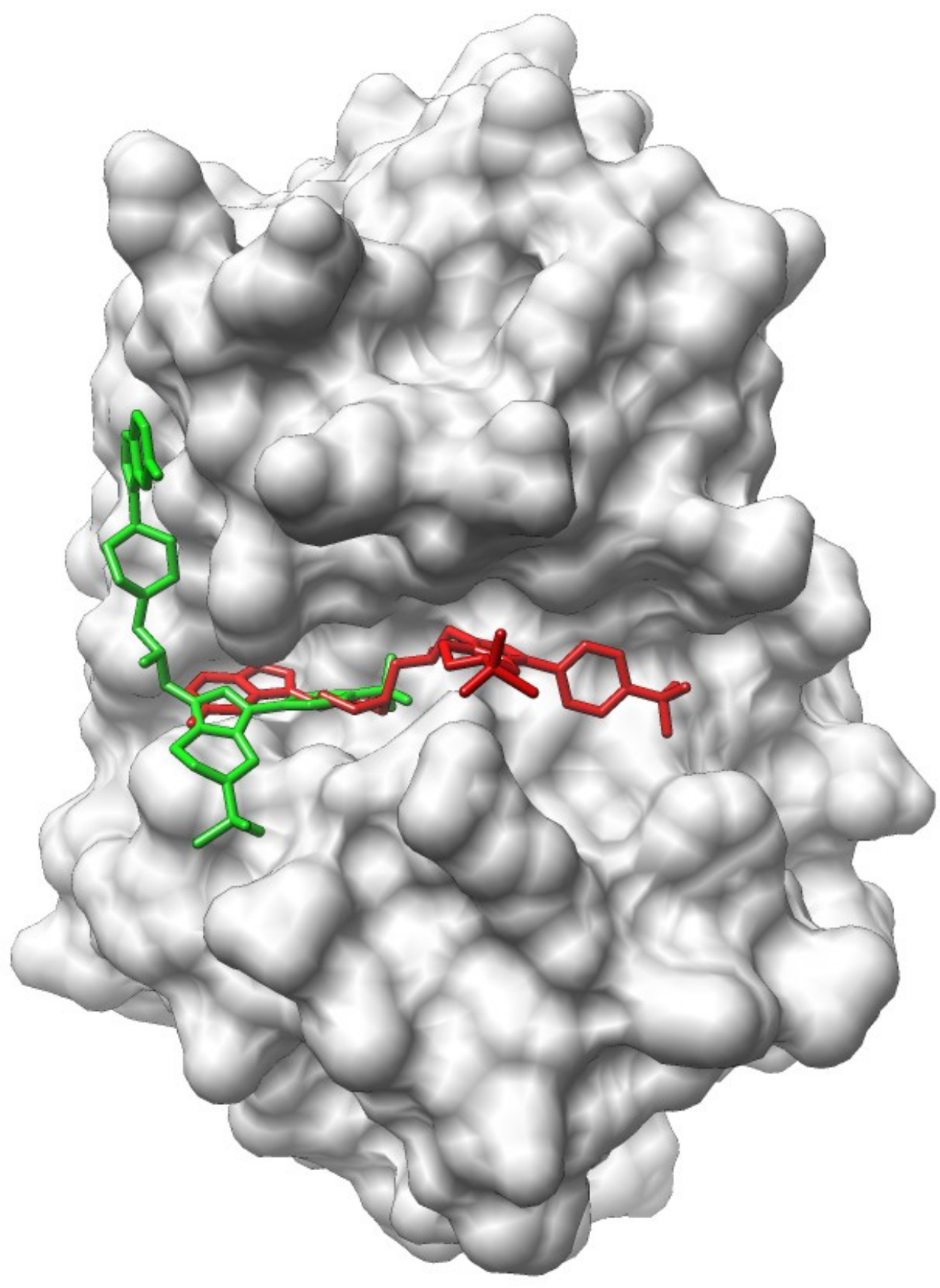

Figure 4.3: The top predicted binding mode (colored red) of CatS_1 in comparison with the binding mode given by the crystal structure (colored green) released by D3R. The protein is shown in surface representation. The ligand (Cast_1) is plotted in stick representation. The predicted binding mode was obtained from the docking method using the bound protein structure 


\subsection{Conclusion}

In this study, we thoroughly analyzed our prediction results on recent D3R challenges, CELPP and GC3. Our binding mode prediction methods have been systematically tested using the large-scale benchmark (more than one thousand targets) provided by CELPP. The results show that current molecular docking methods can benefit from the rapid increase of the number of proteinligand complex structures in the PDB. The use of an appropriate protein structure for docking will significantly improve the success rate of the binding mode prediction. In addition to the docking method, the results of a template-based approach have been assessed. The template-based method achieved better performance than the docking method. However, a drawback of the template-based method is its reliance on the templates, and its performance was poor when there was no good-quality template available. On the other hand, the dockingbased method does not have this issue. Future combination of these two different methods is on the way to improve the binding mode prediction.

\subsection{ACKNOWLEDGEMENT}

Support to XZ from OpenEye Scientific Software Inc. (Santa Fe, NM, http:// www.eyesopen.com) is gratefully acknowledged. This work was supported by NIH R01GM109980 (PI: XZ), NIH R01HL126774 and NIH R01HL142301 (PI: Cui) to XZ. The computations were performed on the high performance computing infrastructure supported by NSF CNS-1429294 (PI: Chi-Ren Shyu) and the HPC resources supported by the University of Missouri Bioinformatics Consortium (UMBC). 


\section{Bibliography}

[1] Kitchen, D. B.; Decornez, H.; Furr, J. R.; Bajorath, J. Nature reviews Drug discovery 2004, 3, 935.

[2] Grinter, S. Z.; Zou, X. Molecules 2014, 19, 10150-10176.

[3] Xu, X.; Huang, M.; Zou, X. Biophysics Reports 2018, 4, 1-16.

[4] Huang, S.-Y.; Grinter, S. Z.; Zou, X. Physical Chemistry Chemical Physics 2010, 12, 12899-12908.

[5] Brooijmans, N.; Kuntz, I. D. Annual review of biophysics and biomolecular structure 2003, 32, 335-373.

[6] Gaieb, Z.; Parks, C. D.; Chiu, M.; Yang, H.; Shao, C.; Walters, W. P.; Lambert, M. H.; Nevins, N.; Bembenek, S. D.; Ameriks, M. K.; Mirzadegan, T.; Burley, S. K.; Amaro, R. E.; Gilson, M. K. Journal of Computer-Aided Molecular Design 2019, 33, 1-18.

[7] Gathiaka, S.; Liu, S.; Chiu, M.; Yang, H.; Stuckey, J. A.; Kang, Y. N.; Delproposto, J.; Kubish, G.; Dunbar, J. B.; Carlson, H. A.; Burley, S. K.; Walters, W. P.; 
Amaro, R. E.; Feher, V. A.; Gilson, M. K. Journal of Computer-Aided Molecular Design 2016, 30, 651-668.

[8] Gaieb, Z.; Liu, S.; Gathiaka, S.; Chiu, M.; Yang, H.; Shao, C.; Feher, V. A.; Walters, W. P.; Kuhn, B.; Rudolph, M. G.; Burley, S. K.; Gilson, M. K.; Amaro, R. E. Journal of Computer-Aided Molecular Design 2017, 32, 1-20.

[9] Berman, H. M. Nucleic Acids Research 2000, 28, 235-242.

[10] Smith, R. D.; Dunbar, J. B.; Ung, P. M.-U.; Esposito, E. X.; Yang, C.-Y.; Wang, S.; Carlson, H. A. Journal of Chemical Information and Modeling 2011, 51, 2115-2131.

[11] Damm-Ganamet, K. L.; Smith, R. D.; Dunbar, J. B.; Stuckey, J. A.; Carlson, H. A. Journal of Chemical Information and Modeling 2013, 53, 1853-1870.

[12] Smith, R. D.; Damm-Ganamet, K. L.; Dunbar, J. B.; Ahmed, A.; Chinnaswamy, K.; Delproposto, J. E.; Kubish, G. M.; Tinberg, C. E.; Khare, S. D.; Dou, J.; Doyle, L.; Stuckey, J. A.; Baker, D.; Carlson, H. A. Journal of Chemical Information and Modeling 2015, 56, 1022-1031.

[13] Carlson, H. A.; Smith, R. D.; Damm-Ganamet, K. L.; Stuckey, J. A.; Ahmed, A.; Convery, M. A.; Somers, D. O.; Kranz, M.; Elkins, P. A.; Cui, G.; Peishoff, C. E.; Lambert, M. H.; Dunbar, J. B. Journal of Chemical Information and Modeling 2016, 56, 1063-1077.

[14] Xu, X.; Yan, C.; Zou, X. Journal of Computer-Aided Molecular Design 2017, 31, 689-699. 
[15] Duan, R.; Xu, X.; Zou, X. Journal of Computer-Aided Molecular Design 2017, 32, 103-111.

[16] Yan, C.; Grinter, S. Z.; Merideth, B. R.; Ma, Z.; Zou, X. Journal of Chemical Information and Modeling 2015, 56, 1013-1021.

[17] Huang, S.-Y.; Zou, X. Journal of Chemical Information and Modeling 2011, 51, 2097-2106.

[18] Huang, S.-Y.; Zou, X. Journal of Chemical Information and Modeling 2011, 51, $2107-2114$.

[19] Grinter, S. Z.; Yan, C.; Huang, S.-Y.; Jiang, L.; Zou, X. Journal of Chemical Information and Modeling 2013, 53, 1905-1914.

[20] Huang, S.-Y.; Zou, X. Proteins: Structure, Function, and Bioinformatics 2006, 66, 399-421.

[21] Trott, O.; Olson, A. J. Journal of computational chemistry 2010, 31, 455-461.

[22] Huang, S.-Y.; Zou, X. Journal of Computational Chemistry 2006, 27, 1866-1875.

[23] Huang, S.-Y.; Zou, X. Journal of computational chemistry 2006, 27, 1876-1882.

[24] Altschul, S. F.; Gish, W.; Miller, W.; Myers, E. W.; Lipman, D. J. Journal of Molecular Biology 1990, 215, 403-410.

[25] Camacho, C.; Coulouris, G.; Avagyan, V.; Ma, N.; Papadopoulos, J.; Bealer, K.; Madden, T. L. BMC bioinformatics 2009, 10, 421. 
[26] Liu, X.; Jiang, H.; Li, H. Journal of Chemical Information and Modeling 2011, $51,2372-2385$.

[27] Hawkins, P. C. D.; Nicholls, A. Journal of Chemical Information and Modeling 2012, 52, 2919-2936.

[28] Hawkins, P. C. D.; Skillman, A. G.; Warren, G. L.; Ellingson, B. A.; Stahl, M. T. Journal of Chemical Information and Modeling 2010, 50, 572-584.

[29] Cheng, T.; Li, X.; Li, Y.; Liu, Z.; Wang, R. Journal of Chemical Information and Modeling 2009, 49, 1079-1093.

[30] Wang, R.; Fang, X.; Lu, Y.; Yang, C.-Y.; Wang, S. Journal of Medicinal Chemistry 2005, 48, 4111-4119.

[31] Pettersen, E. F.; Goddard, T. D.; Huang, C. C.; Couch, G. S.; Greenblatt, D. M.; Meng, E. C.; Ferrin, T. E. Journal of Computational Chemistry 2004, 25, 16051612 .

[32] Efron, B. Breakthroughs in statistics; Springer, 1992; pp 569-593.

[33] Pattengale, N. D.; Alipour, M.; Bininda-Emonds, O. R.; Moret, B. M.; Stamatakis, A. Journal of Computational Biology 2010, 17, 337-354. 


\section{Supplementary}

\section{.1 EDockMS Implementation Details Related to Chapter 3.}

In the development of EDockMS, we aimed to set up a user-friendly docking-based multiple target screening platform as the initial leading step in therapeutic applications.

EDockMS is a docking-based IVS web-server. The platform construction includes four major components, the interface, target database preparation, docking calculations and IVS results analysis.

\section{$.1 .1 \quad$ Server interface}

\section{Input}

The user only needs to upload a mol2 file of the ligand structure. Users also have the options to specify such as job name and e-mail address for their convenience. 


\section{Output}

The Queue page is used for checking the job status. Once a job is submitted successfully, a result page is generated at the same time, users will receive a notification through email after the job is completed. The results are compressed in a tar file which can be downloaded directly. The most promising targets are predicted and ranked by ITScore. The output of each job includes a list of the top 50 protein targets (with disease-related information), and the top 10 binding modes.

\section{.1.2 Database preparation}

The server built-in database MDTD is for storing data that EDockMS needs for multiple target screening. Moreover, MDTD crosslinks to TTD to provide the clinical trial information about the drugs, targets and pathways for therapeutically perspective analysis. First, we used Uniprot $\mathrm{IDs}^{1}$ to extract TTD target relevant $\mathrm{PDBs}^{2}$ because TTD crosslinked UniprotKB and PDB. ${ }^{3}$ Then, we mapped those PDBs to sc-PDB so that the finalized structures in MDTD are the targets that can be tracked with TTD IDs for disease associated details. Last, we used Sphgencpp to generate spheres around the whole surface of each protein in MDTD, and getsph to choose spheres in the vicinity of the protein binding site. Here, we utilized the binding site definitions in sc-PDBs, which are based on the set of amino acid residues that are within 6.5 $\AA$ to any ligand heavy atom. In the current version, we include 3268 protein ligand complexes (3349 distinguish binding sites) associate with 537 target entries. All the PDBs deposited in MDTD corresponding with TTD IDs and correlated information are listed in TableS2. 


\section{.1.3 Docking Protocol}

All of the docking calculations are performed backstage using MDock software (http: //zoulab.dalton.missouri.edu/mdock.htm), details of local installation and usage are introduced in our previous study. ${ }^{4}$ OMEGA is used to generate sets of query ligand conformations (up to 200 conformers as the default setting) for flexible docking (Version 2.5.1. OpenEye Scientific Software, Santa Fe, NM. (http://www . eyesopen. com)).

ITScore is a knowledge-based scoring function (also referred to as statistical potentialbased scoring function) which has been implemented in MDock. The basic idea of the knowledge-based scoring function is to apply Boltzmann distribution principles to extract interaction potentials from the known structural information. Specifically, the pairwise interaction potentials can be expressed as:

$$
u_{i j}(r)=-k_{B} T \ln \left(\rho(r) / \rho^{*}(r)\right)
$$

$i$ and $j$ represent types of atom pairs, $\rho(r)$ is the atom pair density of a protein ligand atom at distance of $\mathrm{r} . \mathrm{r}$ is the distance between the two atoms. $k_{B}$ and $\mathrm{T}$ are the Boltzmann constant and absolute temperature, respectively. Yet, $\rho^{*}(r)$

remains the reference-state hurdle. ${ }^{5}$ The idea of ITScore is utilizing an iterative way to circumvent the reference state problem. ${ }^{5,6}$ Then, the pairwise Energy interaction potentials are generated by the following equations:

$$
u_{i j}^{k+1}(r)=u_{i j}^{k}(r)+\Delta u_{i j}^{k}(r), \quad \Delta u_{i j}^{k}(r)=\frac{1}{2} K_{B} T\left[g_{i j}^{k}(r)-g_{i j}^{o b s}(r)\right]
$$


$g_{i j}^{k}(r)$ is the $k$-th iterative step of pair distribution functions.

\section{.1.4 Clustering procedure}

The server automatically removes redundancies of same family targets. However, for each protein target, it may contain several PDB structures. In order to do the flexible docking, we used all of the structures in the docking procedure. After docking, all of the structures are ranked according to their scores while only the structure with the highest score is kept.

\section{.1.5 Example and validation of the approach}

In terms of accuracy, we have re-docked all of the structures deposited in the database to test the ability of ITScore to predict the binding mode. ITScore achieved a success rate of $84 \%$ on identifying native-like binding modes under the criterion of rmsd $\geq$

$2 \AA$. Table S1 presents the top 5 predicted targets for Progesterone as one of the examples from 47 cases (See in supplementary materials, S3). 
Table S1 related to Chapter3. EDockMS results of Progesterone.

\begin{tabular}{ccccc}
\hline Rank & Predicted target & ITScore & PDB ID & TTD ID \\
\hline 1 & Mineralocorticoid receptor & -58.25 & 2aax & T72168 \\
2 & Progesterone receptor & -55.77 & $1 \mathrm{a} 28$ & T22939 \\
3 & Corticosteroid-binding globulin & -53.73 & $4 \mathrm{bb2}$ & $\mathrm{T} 88452$ \\
4 & Acetylcholinesterase & -53.26 & $4 \mathrm{m0f}$ & $\mathrm{T} 30082$ \\
5 & Androgen receptor & -52.47 & $1 \mathrm{gs} 4$ & $\mathrm{~T} 11211$ \\
\hline
\end{tabular}

The true targets of Progesterone are colored in gray 


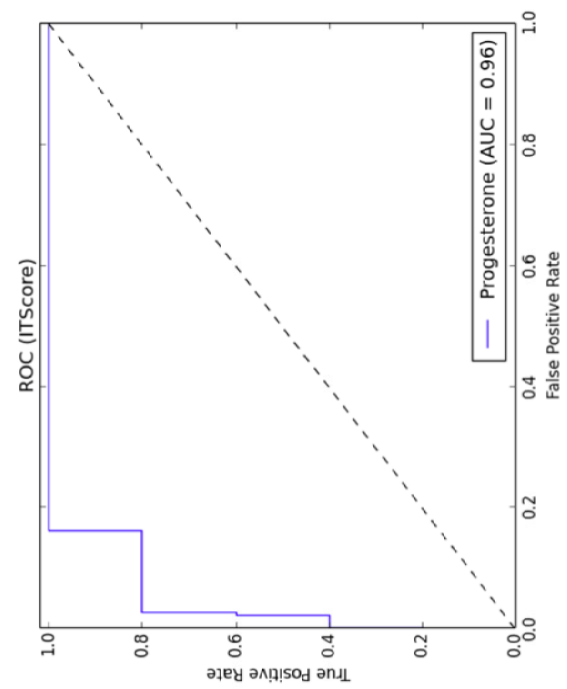

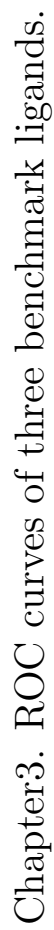

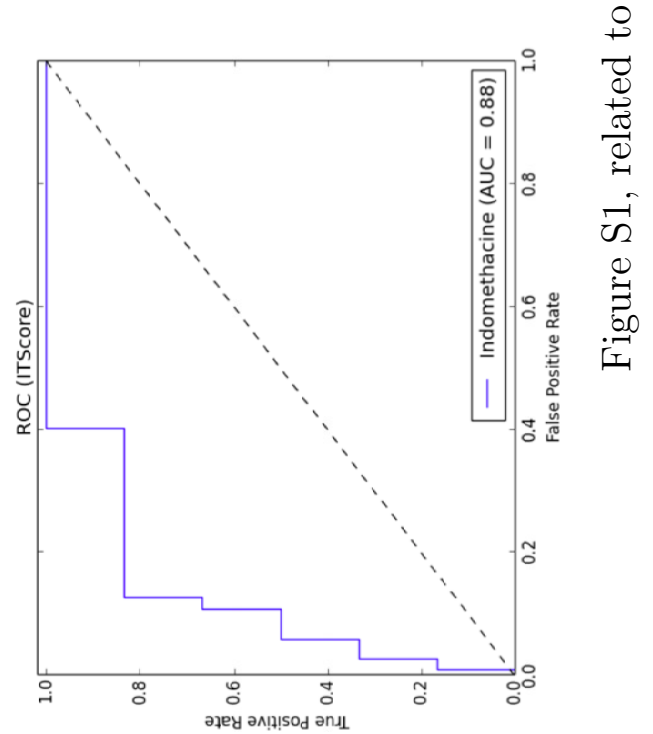




\section{Table S2: related to Chapter3. Target entries in MDTD}

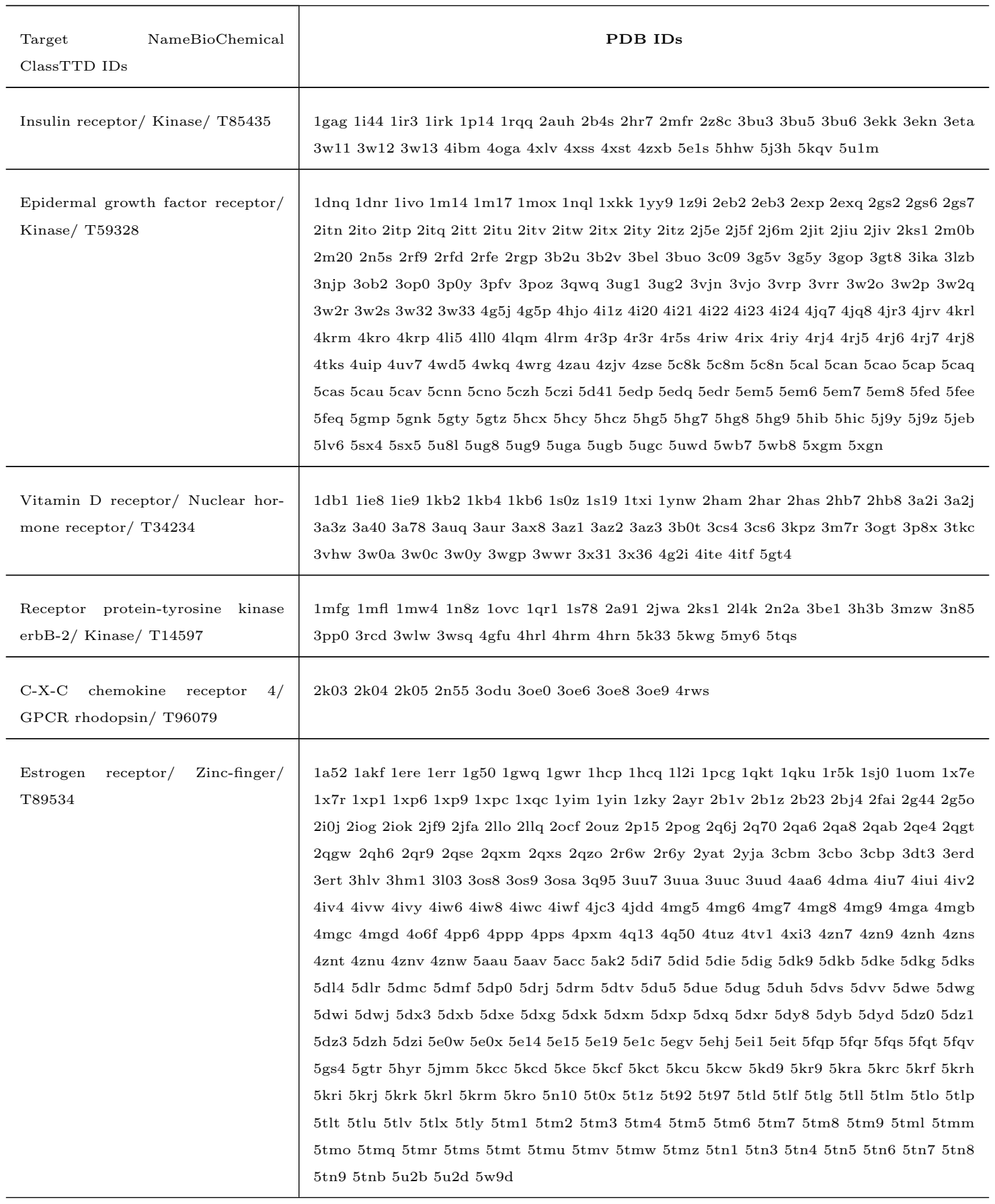




\begin{tabular}{|c|c|}
\hline $\begin{array}{l}\text { Coagulation factor Xa/ Peptidase/ } \\
\text { T84631 }\end{array}$ & 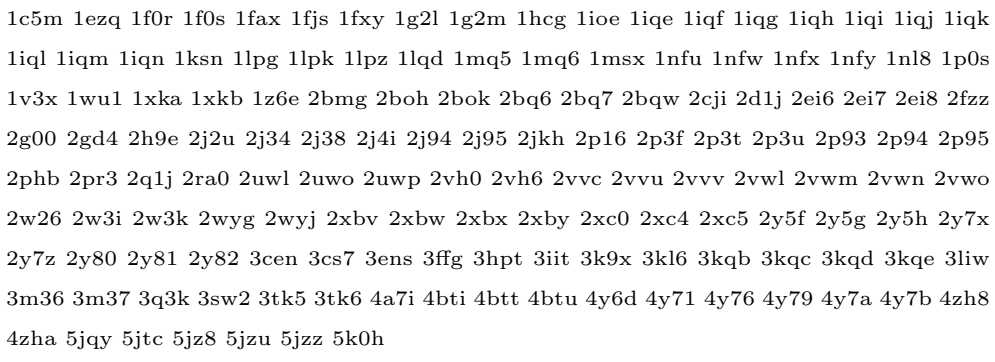 \\
\hline $\begin{array}{l}\text { Vascular endothelial growth factor } \\
\text { receptor } 2 / \text { Kinase/ T } 80975\end{array}$ & $\begin{array}{l}\text { 1vr2 1y6a 1y6b 1ywn 2m59 2met 2meu 2oh4 2p2h 2p2i 2qu5 2qu6 2r15 2x1w 2x1x 2xir } \\
\text { 3b8q 3b8r 3be2 3c7q 3cjf 3cjg 3cp9 3cpb 3cpc 3dtw 3efl 3ewh 3kvq 3s35 3s36 3s37 3u6j } \\
\text { 3v2a 3v6b 3vhe 3vhk 3vid 3vnt 3vo3 3wzd 3wze 4ag8 4agc 4agd 4asd 4ase 5ew3 }\end{array}$ \\
\hline $\begin{array}{l}\text { Fibroblast growth factor receptor } \\
2 / \text { Kinase/ T58454 }\end{array}$ & $\begin{array}{l}\text { 1djs 1e0o 1ev2 1gjo 1ii4 1iil 1nun 1oec 1wvz 2fdb 2psq 2pvf 2pvy 2pwl 2py3 2pz5 2pzp } \\
\text { 2pzr 2q0b 3b2t 3caf 3cly 3cu1 3dar 3euu 3oj2 3ojm 3ri1 4j23 4j95 4j96 4j97 4j98 4j99 } \\
\text { 4wv1 5eg3 5ugl 5ugx 5uhn 5ui0 }\end{array}$ \\
\hline $\begin{array}{l}\text { Androgen receptor/ Zinc-finger/ } \\
\text { T11211 }\end{array}$ & $\begin{array}{l}\text { 1e3g } 1 \text { gs4 } 1 \text { t5z 1t63 } 1 \text { t65 1xj7 1xow 1xq3 } 1 \text { z95 2am9 2ama 2amb 2ao6 2ax6 2ax7 2ax8 } \\
\text { 2ax9 2axa 2hvc 2oz7 2pio 2pip 2piq 2pir 2pit 2piu 2piv 2piw 2pix 2pkl 2pnu 2q7i 2q7j } \\
\text { 2q7k 2q7l 2yhd 2ylo 2ylp 2ylq 2z4j 3b5r 3b65 3b66 3b67 3b68 3btr 313x 313z 3rlj 3rll 3v49 } \\
\text { 3v4a 3zqt 4hlw 4k7a 4oea 4oed 4oey 4oez 4ofr 4ofu 4ogh 4oh5 4oh6 4oha 4oil 4oiu 4oj9 } \\
\text { 4ojb 4ok1 4okb 4okt 4okw 4okx 4olm 4ql8 5cj6 5jjm 5t8e 5t8j 5v8q }\end{array}$ \\
\hline $\begin{array}{l}\text { Nitric-oxide synthase, endothelial/ } \\
\text { Oxidoreductases acting on paired } \\
\text { donors/ T06046 }\end{array}$ & $\begin{array}{l}1 \mathrm{~m} 9 \mathrm{j} 1 \mathrm{~m} 9 \mathrm{k} 1 \mathrm{~m} 9 \mathrm{~m} 1 \mathrm{~m} 9 \mathrm{q} 1 \mathrm{~m} 9 \mathrm{r} 1 \mathrm{niw} 21172 \mathrm{mg} 52 \mathrm{n} 8 \mathrm{j} 3 \mathrm{eah} 3 \mathrm{nos} 4 \mathrm{~d} 1 \mathrm{o} 4 \mathrm{~d} 1 \mathrm{p} 5 \mathrm{uo} 85 \mathrm{uo9} 5 \text { uoa } \\
5 \text { uob } 5 \text { uoc } 5 \mathrm{vvb} 5 \mathrm{vvc} 5 \mathrm{vvd} 5 \mathrm{xof}\end{array}$ \\
\hline $\begin{array}{l}\text { B-Raf proto-oncogene serine/ } \\
\text { threonine-protein kinase/ T99089 }\end{array}$ & 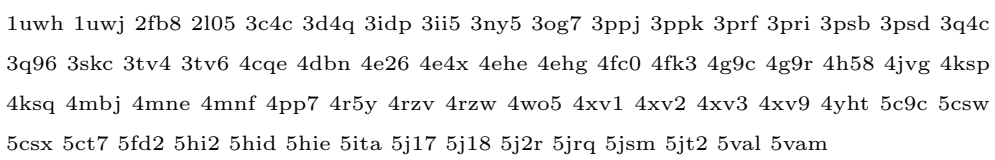 \\
\hline
\end{tabular}


Coagulation factor IIa/ Peptidase/ T94033 1a2c 1a3b 1a3e 1a46 1a4w 1a5g 1a61 1abi 1abj 1ad8 1ae8 1afe 1aht 1ai8 1aix 1awf 1awh 1ay6 1b5g 1b7x 1ba8 1bb0 1bcu 1bhx 1bmm 1bmn 1bth 1c1u 1c1v 1c1w 1c4u 1c4v 1c4y 1c5l 1c5n 1c5o 1ca8 1d3d 1d3p 1d3q 1d3t 1d4p 1d6w 1d9i 1de7 1dit 1dm4 1doj 1dwb 1dwc 1dwd 1dwe 1dx5 1e0f 1eb1 1eoj 1eol 1fpc 1fph 1 g30 1g32 1 g37 1 ghv 1 ghw 1 ghx 1ghy 1gj4 1gj5 1h8d 1h8i 1hag 1hah 1hai 1hao 1hap 1hbt 1hdt 1hgt 1hlt 1hut 1hxe 1 hxf 1ihs 1 iht 1 jmo 1 jou 1 jwt $1 \mathrm{k} 21$ k 22 1kts $1 \mathrm{ktt} 1 \mathrm{hc} 1$ hd 1 he $1 \mathrm{lhf} 1 \mathrm{hg} 1 \mathrm{mh} 01 \mathrm{mu} 61 \mathrm{mu} 8$ 1mue 1nm6 1no9 1nrn 1nro 1nrp 1nrq 1nrr 1nrs 1nt1 1nu7 1nu9 1ny2 1nzq 1o0d 1o2g 1o5g 1ook 1oyt 1p8v 1ppb 1qbv 1qhr 1qj1 1qj6 1qj7 1qur 1rd3 1riw 1sb1 1sfq 1sg8 1sgi $1 \mathrm{shh} 1 \mathrm{sl} 31 \mathrm{sr} 51 \mathrm{t} 4 \mathrm{u} 1 \mathrm{t} 4 \mathrm{v} 1 \mathrm{ta} 21 \mathrm{ta} 61 \mathrm{tb} 6$ tbz 1 thp 1 thr 1 ths 1 tmb 1 tmt 1 tmu 1 tom 1 tq0 1 tq7 1twx 1uma 1uvs 1vr1 1vzq 1w7g 1way 1wbg 1xm1 1xmn 1ype 1ypg 1ypj 1ypk 1ypl 1ypm 1z71 1z8i 1z8j 1zgi 1zgv 1zrb 2a0q 2a2x 2a45 2afq 2ank 2anm 2b5t 2bdy 2bvr 2bvs 2 bvx 2 bxt 2bxu 2c8w 2c8x 2c8y 2c8z 2c90 2c93 2cf8 2cf9 2cn0 2feq 2fes 2gde 2gp9 2h9t 2hgt 2hnt 2hpp 2hpq 2hwl 2jh0 2jh5 2jh6 2od3 2pgb 2pgq 2pks 2pw8 2r2m 2thf 2uuf 2uuj 2uuk 2v3h 2v3o 2zc9 2zda 2zdv 2zfo 2zff 2zfp 2zfq 2zfr 2zg0 2zgb 2zgx 2zhe 2zhf 2zhq 2zhw 2zi2 2ziq 2znk 2zo3 3b23 3b9f 3bef 3bei 3bf6 3biu 3biv 3bv9 3c1k 3c27 3d49 3da9 3dd2 3dhk 3dt0 3dux 3e6p 3ee0 3egk 3eq0 3f68 3gic 3gis 3hat 3hkj 3htc 3jz1 3jz2 3k65 3ldx 3lu9 3nxp 3p17 3p6z 3p70 3pmh 3po1 3qdz 3qgn 3qlp 3qto 3qtv 3qwc 3qx5 3r3g 3rlw 3rly 3rm0 3rm2 3rml 3rmm 3rmn 3rmo 3s7h 3s7k 3sha 3shc 3si3 3si4 3sqe 3sqh 3sv2 3t5f 3tu7 3u69 3u8o 3u8r 3u8t 3u98 3u9a 3utu 3uwj 3vxe 3vxf 4ax9 4ayv 4ayy 4az2 4bah 4bak 4bam 4ban 4bao 4baq 4boh 4ch2 4ch8 4dih 4dii 4dt7 4dy7 4e05 4e06 4e7r 4h6s 4h6t 4hfp 4htc 4hzh 4i7y 4loy 4lxb 4lz1 4lz4 4mlf 4n3l 4nze 4nzq 4o03 4rkj 4rko 4rn6 4thn 4ud9 4udw 4ue7 4ueh 4ufd 4ufe 4uff 4ufg 4yes 5a2m 5af9 5afy 5afz 5ahg 5cmx 5do4 5e8e 5edk 5edm 5ew1 5ew2 5gds 5gim 5jdu 5jfd 5jzy 5l6n 5nhu 5to3 6eo6 6eo7 7kme 8kme

RAC serine/ threonine-protein kinase/ T67619

1h10 1unp 1unq 1unr 2uvm 2uzr 2uzs 3cqu 3cqw 3mv5 3mvh 3o96 3ocb 3ow4 3qkk 3qkl $3 \mathrm{qkm} 4 \mathrm{ejn} 4 \mathrm{ekk} 4 \mathrm{ekl} 4 \mathrm{gv} 15 \mathrm{kcv}$

mRNA of RAF proto-oncogene serine/ threonine-protein kinase/ T60405

1c1y 1faq 1far 1gua 1rfa 3cu8 3iqj 3iqu 3iqv 3kuc 3kud 3nkx 3o8i 3omv 4fj3 4g0n 4g3x 4 iea 4 ihl

Peroxisome proliferator activated receptor gamma/ Nuclear hormone receptor/ T58921

$1 \mathrm{fm} 61 \mathrm{fm} 9$ 1i7i $1 \mathrm{k} 74$ 1 knu 1nyx 1prg 1rdt 1wm0 1zeo 1zgy 2ath 2f4b 2fvj 2g0g 2g0h 2gtk 2hfp 2hwq 2hwr 2i4j 2i4p 2i4z 2om9 2p4y 2pob 2prg 2q59 2q5p 2q5s 2q61 2q6r 2q6s 2q8s 2qmv 2vsr 2vst 2vv0 2vv1 2vv2 2vv3 2vv4 2xkw 2yfe 2zk0 2zk1 2zk2 2zk3 2zk4 2zk5 2zk6 2zno 2zvt 3ads 3adt 3adu 3adv 3adw 3adx 3an3 3an4 3b0q 3b0r 3b1m 3b3k 3bc5 3cdp 3cds 3cs8 3cwd 3d6d 3dzu 3dzy 3e00 3et0 3et3 3fej 3fur 3g9e 3gbk 3h0a 3ho0 3hod 3ia6 3k8s 3kmg 3lmp 3noa 3osi 3osw 3pba 3po9 3prg 3qt0 3r5n 3r8a 3r8i 3s9s 3sz1 3t03 3ty0 3u9q 3v9t 3v9v 3v9y 3vjh 3vji 3vn2 3vso 3vsp 3wj4 3wj5 3wmh 3x1h 3x1i 4a4v 4a4w 4ci5 4e4k 4e4q 4em9 4ema 4f9m 4fgy 4hee 4jaz 4j14 41964198 4o8f 4oj4 4prg 4pvu 4pwl 4r06 4r2u 4r6s 4xld 4xta 4xuh 4xum 4y29 4yt1 5azv 5dsh 5dv3 5dv6 5dv8 5dvc 5dwl 5f9b 5gtn 5 to 5 gtp 5 hzc 5 ji0 5lsg 5tto 5two 5 u 51

1gkc 1gkd 1itv 1l6j 1lkg 2ovx 2ovz 2ow0 2ow1 2ow2 4h1q 4h2e 4h3x 4h82 4hma 4jij 4jqg 4 wzv 4 xct 5cuh 5i12 5th6 5th9 5ue3 5ue4

3-hydroxy-3-methylglutaryl1dq8 1dq9 1dqa 1hw8 1hw9 1hwi 1hwj 1hwk 1hwl 2q1l 2q6b 2q6c 2r4f 3bgl 3cct 3ccw 3ccz $3 \mathrm{~cd} 03 \mathrm{~cd} 53 \mathrm{~cd} 73 \mathrm{cda} 3 \mathrm{cdb}$ 


\begin{tabular}{|c|c|}
\hline $\begin{array}{l}\text { Basic fibroblast growth factor re- } \\
\text { ceptor } 1 / \text { Kinase/ T47101 }\end{array}$ & $\begin{array}{l}\text { 1agw 1cvs 1evt } 1 \text { fgi } 1 \text { fgk } 1 \text { fq9 } 1 \text { xr0 } 2 \mathrm{cr} 3 \text { 2fgi 3c4f 3dpk 3gqi 3gql 3js2 3krj 3krl 3kxx 3ky2 } \\
\text { 3ojv 3rhx 3tt0 4f63 4f64 4f65 4nk9 4nka 4nks 4rwi 4rwj 4rwk 4rwl 4uwb 4uwc 4uwy 4v01 } \\
4 \text { v04 4v05 4wun 4zsa 5a46 5a4c 5am6 5am7 5b7v 5ew8 5flf 5uq0 5ur1 5vnd 5w59 }\end{array}$ \\
\hline $\begin{array}{l}\text { Estradiol } 17 \text { beta-dehydrogenase } 1 / \\
\text { Short-chain dehydrogenases reduc- } \\
\text { tases / T44011 }\end{array}$ & 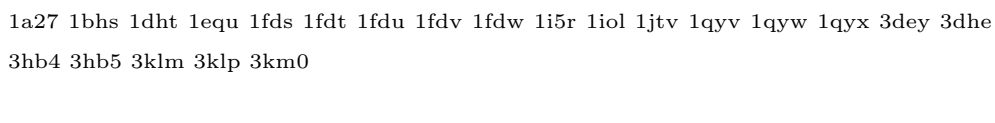 \\
\hline $\begin{array}{l}\text { Glucocorticoid receptor/ Zinc- } \\
\text { finger/ T40016 }\end{array}$ & $\begin{array}{l}\text { 1m2z 1nhz 1p93 3bqd 3cld 3e7c 3h52 3k22 3k23 4csj 4hn5 4hn6 4lsj 4mdd 4p6w 4p6x } \\
4 \text { udc 4udd 5cbx 5cby 5cbz 5cc1 5e69 5e6a 5e6b 5e6c 5e6d 5emc 5emp 5emq 5g3j 5g5w } \\
5 \text { nfp } 5 \text { nft } 5 \text { va0 } 5 \text { va7 }\end{array}$ \\
\hline $\begin{array}{l}\text { Apoptosis regulator } \mathrm{Bcl}-2 / \mathrm{Bcl}-2 \\
\text { family/ T31309 }\end{array}$ & 1g5m 1gjh 1ysw $2 \mathrm{o} 212 \mathrm{o} 22$ 2o2f 2 w31 2 xa0 4 aq3 4 ieh 4 lvt 4 lxd 4 man 5 agw 5 agx 5 fcg 5 jsn \\
\hline $\begin{array}{l}\text { Cytochrome P450 } 19 / \text { Oxidoreduc- } \\
\text { tases acting on paired donors/ } \\
\text { T13260 }\end{array}$ & 1tqa 3 eqm $3 \mathrm{~s} 79$ 3s7s $4 \mathrm{gl} 54 \mathrm{gl} 17$ 4kq8 $5 \mathrm{jkv} 5 \mathrm{jkw} 5 \mathrm{j} 16 \quad 5 \mathrm{jl} 17$ 5j19 \\
\hline $\begin{array}{l}\text { C-C chemokinereceptor type 5/ } \\
\text { GPCR rhodopsin/ T09960 }\end{array}$ & 1nd8 1ne0 1opn 1opt 1opw $21872 \mathrm{mzx} 2$ rll $2 \mathrm{rrs} 4 \mathrm{mbs}$ \\
\hline $\begin{array}{l}\text { Adenosine deaminase/ Carbon- } \\
\text { nitrogen hydrolase/ T03661 }\end{array}$ & $1 \mathrm{~m} 7 \mathrm{~m} 3 \mathrm{iar}$ \\
\hline mRNA of HER2/ Kinase/ T96788 & 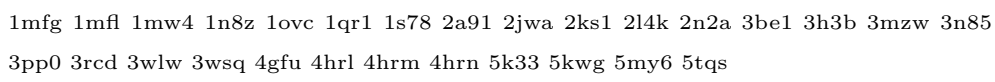 \\
\hline $\begin{array}{l}\text { Angiopoietin } 1 \text { receptor/ Kinase/ } \\
\text { T92144 }\end{array}$ & 1 fvr $2 \mathrm{gy} 52 \mathrm{gy} 72 \mathrm{oo} 82 \mathrm{osc} 2 \mathrm{p} 4 \mathrm{i} 2 \mathrm{wqb} 3$ bea $318 \mathrm{p} 4 \mathrm{k} 0 \mathrm{v} 4 \mathrm{x} 3 \mathrm{j} 5$ mya 5 myb 5 utk \\
\hline $\begin{array}{l}\text { mRNA of Protein tyrosine } \\
\text { phosphatase-1B/ Target of an- } \\
\text { tisense drug/ T89529 }\end{array}$ & 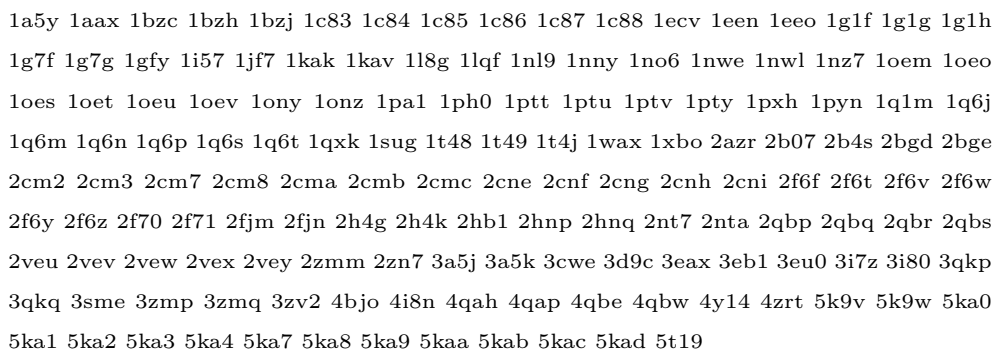 \\
\hline Glucokinase/ Kinase/ T87166 & 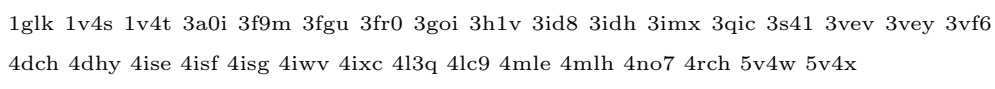 \\
\hline Stromelysin-1/ Peptidase/ T86702 & $\begin{array}{l}\text { 1b3d 1b8y 1biw 1bm6 1bqo 1c3i 1c8t 1caq 1ciz 1cqr 1d5j 1d7x 1d8f 1d8m 1g05 1g49 1g4k } \\
\text { 1hfs } 1 \text { hy } 7 \text { 1m1w 1oo9 1qia 1qic 1slm 1sln 1uea 1ums 1umt 1usn 2d1o 2jnp 2jt5 2jt6 2srt } \\
\text { 2usn 3ohl 3oho 3usn 4dpe 4g9l 4ja1 }\end{array}$ \\
\hline
\end{tabular}




\begin{tabular}{|c|c|}
\hline $\begin{array}{l}\text { Proto-oncogene tyrosine-protein ki- } \\
\text { nase SRC/ Kinase/ T } 85943\end{array}$ & $\begin{array}{l}\text { 1a07 1a08 1a09 1a1a 1a1b 1a1c 1a1e 1fmk 1hcs 1hct 1ksw 1o41 1o42 1o43 1o44 1o45 1o46 } \\
1 \mathrm{o} 471 \mathrm{o} 48 \text { lo49 1o4a 1o4b 1o4c 1o4d 1o4e 1o4f 1o4g 1o4h 1o4i 1o4j 1o4k 1o4l 1o4m 1o4n } \\
\text { 1o4o 1o4p 1o4q 1o4r 1shd 1y57 1yi6 1yoj 1yol 1yom 2bdf 2bdj 2h8h 2src 3vro 3zmp 3zmp } \\
4 \text { f59 4f5a 4f5b 4hxj 4k11 4mxo 4mxx 4mxy 4mxz }\end{array}$ \\
\hline $\begin{array}{l}\text { Purine nucleoside phosphorylase/ } \\
\text { Pentosyltransferase/ T78198 }\end{array}$ & $\begin{array}{l}\text { 1m73 1pf7 1pwy 1rct 1rfg 1rr6 1rsz 1rt9 1ula 1ulb 1v2h 1v3q 1v41 1v45 1yry 2a0w 2a0x } \\
\text { 2a0y 2oc4 2oc9 2on6 2q7o 3bgs 3d1v 3gb9 3ggs 3iny 3k8o 3k8q 3phb 4ear 4eb8 4ece 4gka } \\
\text { 5etj 5ugf }\end{array}$ \\
\hline $\begin{array}{l}\text { Adenosine A2a receptor/ GPCR } \\
\text { rhodopsin/ T77365 }\end{array}$ & $\begin{array}{l}1 \mathrm{mmh} 1 \text { upe } 2 \mathrm{ydo} 2 \mathrm{ydv} 3 \mathrm{eml} 3 \mathrm{pwh} 3 \mathrm{qak} 3 \mathrm{rey} 3 \mathrm{rfm} 3 \mathrm{uza} 3 \mathrm{uzc} 3 \mathrm{vg} 93 \mathrm{vga} 4 \mathrm{eiy} 4 \mathrm{ug} 24 \mathrm{uhr} \\
5 \mathrm{~g} 535 \mathrm{iu} 45 \mathrm{iu} 75 \mathrm{iu} 85 \mathrm{iua} 5 \mathrm{iub} 5 \mathrm{jtb} 5 \mathrm{k} 2 \mathrm{a} 5 \mathrm{k} 2 \mathrm{~b} 5 \mathrm{k} 2 \mathrm{c} 5 \mathrm{k} 2 \mathrm{~d} 5 \mathrm{mzj} 5 \mathrm{mzp} 5 \mathrm{n} 2 \mathrm{r} 5 \mathrm{nlx} 5 \mathrm{~nm} 25 \mathrm{~nm} 4 \\
5 \text { uig 5uvi }\end{array}$ \\
\hline $\begin{array}{l}\text { Mitogen-activated protein kinase } \\
\text { 14/ Kinase/ T65864 }\end{array}$ & 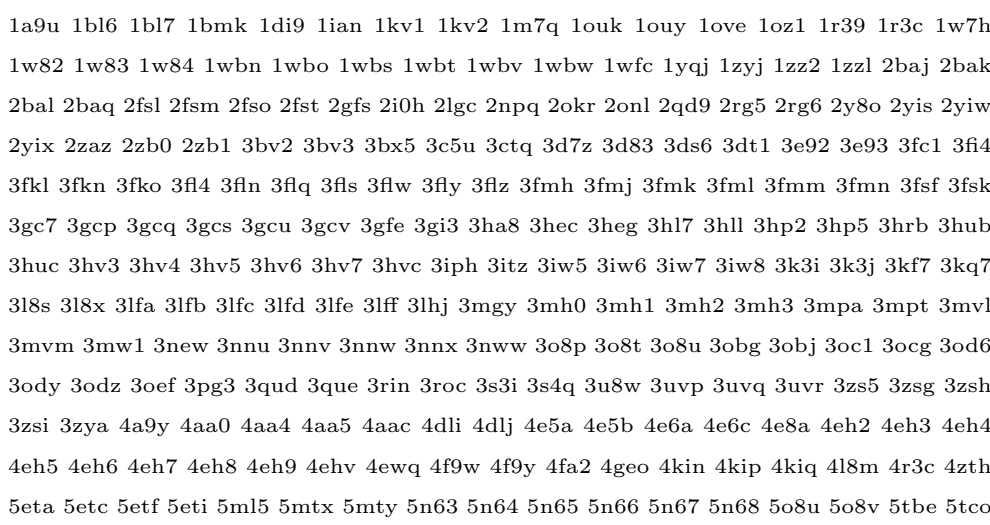 \\
\hline $\begin{array}{l}\text { Aldo-keto reductase family } 1 \text { mem- } \\
\text { ber C3/ Short-chain dehydroge- } \\
\text { nases reductases/ T60857 }\end{array}$ & $\begin{array}{l}\text { 1ry0 1ry8 1s1p 1s1r 1s2a 1s2c 1xf0 1zq5 2f38 2fgb 3r43 3r58 3r6i 3r7m 3r8g 3r8h 3r94 } \\
3 \text { ufy 3ug8 3ugr 3uwe 4dbs } 4 \text { dbu } 4 \text { dbw } 4 \text { dz5 } 4 \text { fa } 34 \text { fal } 4 \text { fam } 4 \text { h7c } 4 \text { hmn } 4 \text { wdt } 4 \text { wdu } 4 \text { wdw } \\
4 \text { wdx } 4 \text { wrh } 4 \text { xvd } 4 \text { xve } 4 \text { yvv } 4 \text { yvx } 4 \text { zfc } 5 \text { hnt } 5 \text { hnu } 5 \text { jm5 }\end{array}$ \\
\hline $\begin{array}{l}\text { Mitogen-activated protein kinase 1/ } \\
\text { Kinase/ T58970 }\end{array}$ & 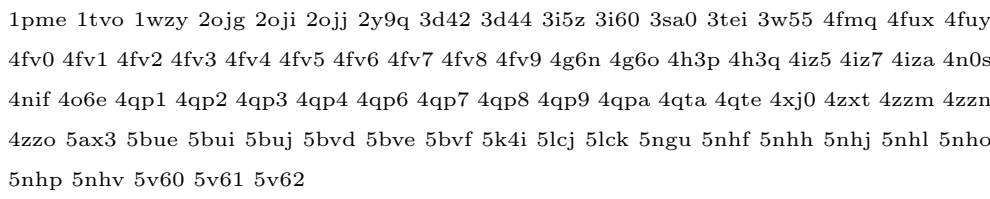 \\
\hline Apopain/ Peptidase/ T57943 & $\begin{array}{l}\text { 1cp3 1gfw 1i3o 1nme 1nmq 1nms 1pau 1qx3 1re1 1rhj 1rhk 1rhm 1rhq 1rhr 1rhu 2c1e } \\
\text { 2c2k 2c2m 2c2o 2cdr 2cjx 2cjy 2cnk 2cnl 2cnn 2cno 2dko 2h5i 2h5j 2h65 2j30 2j31 2j32 } \\
2 \mathrm{j} 33 \text { 2xyg 2xyh 2xyp 2xzd 2xzt 2y0b 3deh 3dei 3dej 3dek 3edq 3gjq 3gjr 3gjs 3gjt 3h0e } \\
3 \text { itn 3kjf 3pcx 3pd0 3pd1 4dcj 4dco 4dcp 4eha 4ehd 4ehf 4ehh 4ehk 4ehl 4ehn 4jje 4jqy } \\
\text { 4jqz 4jr0 4pry 4ps0 4qtx 4qty 4qu0 4qu5 4qu8 4qu9 4qua 4qub 4qud 4que 4qug 4quh 4qui } \\
\text { 4quj 4qul 5i9b 5i9t 5iab 5iae 5iag 5iaj 5iak 5ian 5iar 5ias 5ibc 5ibp 5ibr 5ic4 }\end{array}$ \\
\hline $\begin{array}{l}\text { Beta-2 adrenergic receptor/ GPCR } \\
\text { rhodopsin/ T52522 }\end{array}$ & $\begin{array}{l}\text { 1gq4 2r4r 2r4s 2rh1 3d4s 3kj6 3ny8 3ny9 3nya 3p0g 3pds 3sn6 4gbr 4lde 4ldl 4ldo 4qkx } \\
5 \mathrm{~d} 5 \mathrm{a} 5 \mathrm{~d} 5 \mathrm{~b} 5 \mathrm{~d} 615 \mathrm{jqh} 5 \mathrm{x} 7 \mathrm{~d}\end{array}$ \\
\hline
\end{tabular}




\begin{tabular}{|c|c|}
\hline $\begin{array}{l}\text { Interstitial collagenase/ Peptidase/ } \\
\text { T52450 }\end{array}$ & 1ayk 1cge $1 \mathrm{cgf} 1 \mathrm{cgl} 1 \mathrm{hfc} 1 \mathrm{su} 32 \mathrm{ayk} 2 \mathrm{clt} 2 \mathrm{j} 0 \mathrm{t} 2 \mathrm{tcl} 3 \mathrm{ayk} 3 \mathrm{shi} 4$ auo 4 ayk $966 \mathrm{c}$ \\
\hline $\begin{array}{l}\text { Bile acid receptor/ Nuclear hor- } \\
\text { mone receptor/ T5 } 51426\end{array}$ & $\begin{array}{l}\text { 1osh 1osk 3bej 3dct 3dcu 3fli 3fxv 3gd2 3hc5 3hc6 3l1b 3okh 3oki 3olf 3omk 3omm 3oof } \\
\text { 3ook 3p88 3p89 3rut 3ruu 3rvf 4oiv 4qe6 4qe8 4wvd 5iaw 5ick 5q0i 5q0j 5q0k 5q01 5q0m } \\
\text { 5q0n 5q0o 5q0p 5q0q 5q0r 5q0s 5q0t 5q0u 5q0v 5q0w 5q0x 5q0y 5q0z 5q10 5q11 5q12 } \\
\text { 5q13 5q14 5q15 5q16 5q17 5q18 5q19 5q1a 5q1b 5q1c 5q1d 5q1e 5q1f 5q1g 5q1h 5q1i }\end{array}$ \\
\hline $\begin{array}{l}\text { Muscarinic acetylcholine receptor } \\
\text { M2/ GPCR rhodopsin/ T46185 }\end{array}$ & 1lub 3uon $4 \mathrm{mqs} 4 \mathrm{mqt}$ \\
\hline $\begin{array}{l}\text { Xanthine dehydrogenase/ oxidase/ } \\
\text { T40954 }\end{array}$ & $2 \mathrm{ckj} 2 \mathrm{e} 1 \mathrm{q}$ \\
\hline $\begin{array}{l}\text { Glyceraldehyde } \\
\text { dehydrogenase, } \\
\text { Glyceraldehyde-3-phosphate/ } \\
\text { T39321 }\end{array}$ & 1u8f 1 znq 2 feh 3 gpd 4 wnc 4 wni \\
\hline $\begin{array}{l}\text { Acetylcholinesterase/ Carboxylic } \\
\text { ester hydrolase/ T30082 }\end{array}$ & $\begin{array}{l}\text { 1b41 } 1 \text { f8u } 1 \text { puv } 1 \text { puw } 1 \mathrm{vzj} 2 \mathrm{clj} 2 \mathrm{x} 8 \mathrm{~b} 3 \text { lii } 4 \mathrm{bdt} 4 \mathrm{ey} 44 \mathrm{ey} 54 \text { ey } 64 \text { ey } 74 \text { ey } 8 \text { 4m0e } 4 \mathrm{~m} 0 \mathrm{f} 4 \mathrm{pqe} \\
5 \text { foq } 5 \text { fpq } 5 \mathrm{hf} 55 \mathrm{hf} 65 \mathrm{hf} 85 \mathrm{hf} 95 \mathrm{hfa}\end{array}$ \\
\hline $\begin{array}{l}\text { Progesterone receptor/ Zinc-finger/ } \\
\text { T22939 }\end{array}$ & $\begin{array}{l}\text { 1a28 1e3k 1sqn } 1 \text { sr7 1zuc 2c7a 2ovh 2ovm 2w8y 3d90 3g8o 3hq5 3kba 3zr7 3zra 3zrb 4a } 2 \mathrm{j} \\
4 \mathrm{apu} 4 \mathrm{oar} 5 \mathrm{cc} 0\end{array}$ \\
\hline $\begin{array}{l}\text { Tumor necrosis factor/ Cytokine: } \\
\text { tumor necrosis factor/ T20178 }\end{array}$ & $\begin{array}{l}\text { 1a8m } 1 \text { tnf } 2 \text { az5 } 2 \text { e7a } 2 \text { tun } 2 \text { zjc } 2 \text { zpx 3alq } 3 i t 8319 j 3 \text { wd5 } 4 \mathrm{~g} 3 \mathrm{y} 4 \text { tsv } 4 \text { twt } 4 \text { y6o } 5 \mathrm{~m} 2 \mathrm{i} 5 \mathrm{~m} 2 \mathrm{j} \\
5 \mathrm{~m} 2 \mathrm{~m} 5 \mathrm{mu} 85 \text { tsw } 5 \text { uui } 5 \text { wux }\end{array}$ \\
\hline $\begin{array}{l}\text { Urokinase-type plasminogen activa- } \\
\text { tor/ Peptidase/ T17758 }\end{array}$ & 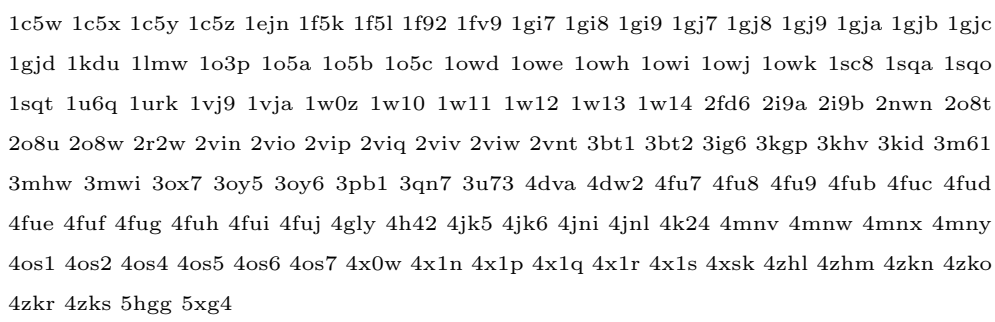 \\
\hline Cellular tumor antigenp53/ T15739 & 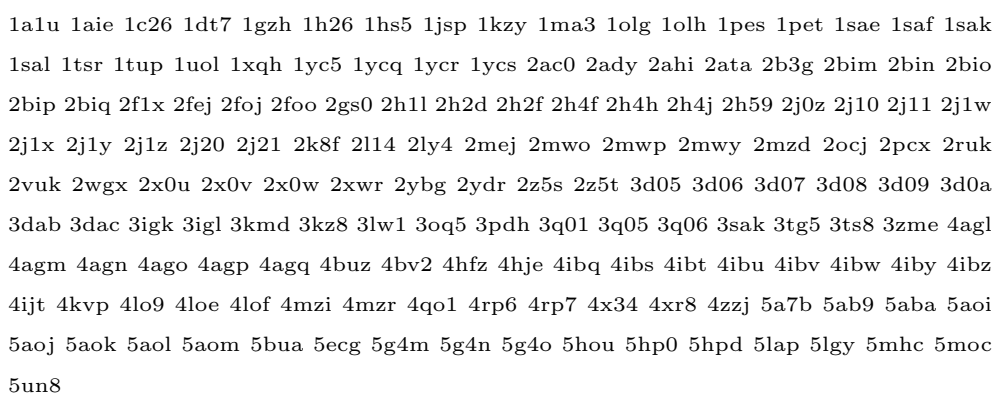 \\
\hline
\end{tabular}




\begin{tabular}{|c|c|}
\hline $\begin{array}{l}\text { Protein kinase C, alpha type/ Ki- } \\
\text { nase/ T12808 }\end{array}$ & 2eli 3iw4 4dnl 4ra4 \\
\hline $\begin{array}{l}\text { Lck tyrosine kinase/ Kinase/ } \\
\text { T12499 }\end{array}$ & 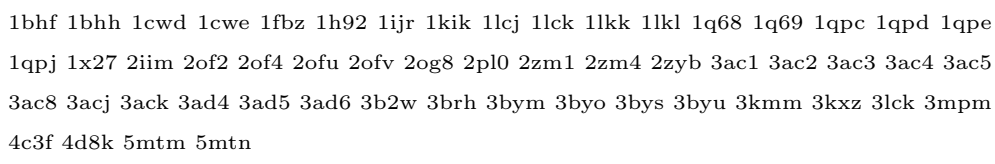 \\
\hline $\begin{array}{l}\text { Glutamate carboxypeptidase II/ } \\
\text { Peptidase/ T } 97071\end{array}$ & 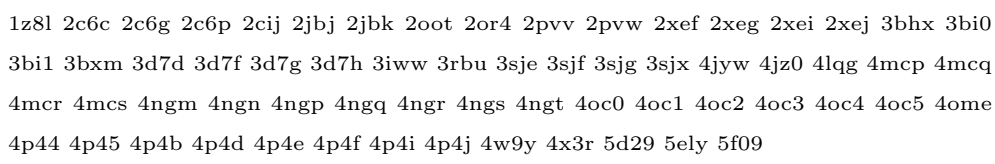 \\
\hline $\begin{array}{l}\text { Cell division protein kinase } 6 / \mathrm{Ki}- \\
\text { nase/ T89361 }\end{array}$ & 1bi7 1bi8 1blx 1g3n 1jow 1xo2 2euf 2f2c 3nup 3nux 4aua 4ez5 4tth 512i 512s 512t \\
\hline $\begin{array}{l}\text { Peroxisome proliferator acti- } \\
\text { vated receptor alpha/ Zinc-finger/ } \\
\text { T86591 }\end{array}$ & $\begin{array}{l}\text { 1i7g } 1 \mathrm{k} 7 \mathrm{l} 1 \mathrm{kkq} 2 \mathrm{npa} 2 \mathrm{p} 54 \text { 2rew 2znn } 3 \text { et1 3fei 3g8i 3kdt 3kdu 3sp6 3vi8 4bcr 4ci4 5azt } \\
5 \text { hyk }\end{array}$ \\
\hline $\begin{array}{l}\text { Sphingosine kinase/ Kinase/ } \\
\text { T86014 }\end{array}$ & $3 \mathrm{vzb} 3 \mathrm{vzc} 3 \mathrm{vzd} 41024 \mathrm{v} 24$ \\
\hline $\begin{array}{l}\text { Inhibitor of apoptosis protein 2/ } \\
\text { Carbon-nitrogen ligase/ } \mathrm{T} 84780\end{array}$ & $\begin{array}{l}\text { 1qbh } 219 \mathrm{~m} 3 \mathrm{~d} 9 \mathrm{t} 3 \mathrm{~d} 9 \mathrm{u} 3 \mathrm{~m} 1 \mathrm{~d} 3 \mathrm{mup} 3 \mathrm{oz} 13 \mathrm{t} 6 \mathrm{p} 3 \mathrm{uw} 4 \text { eb9 4hy4 4hy5 } 4 \mathrm{kmn} 4 \text { lge } 4 \text { lgu } 4 \mathrm{mti} \\
4 \mathrm{mu} 75 \mathrm{~m} 6 \mathrm{n}\end{array}$ \\
\hline $\begin{array}{l}\text { Amine oxidase [flavin-containing] } \\
\mathrm{B} / \text { Oxidoreductases acting on } \mathrm{CH}- \\
\mathrm{NH} 2 \text { group of donors/ T83011 }\end{array}$ & $\begin{array}{l}\text { 1gos 1h8r 1oj9 1oja 1ojc 1ojd 1s2q 1s2y 1s3b 1s3e 2bk3 2bk4 2bk5 2byb 2c64 2c65 2c66 } \\
\text { 2c67 2c70 2c72 2c73 2c75 2c76 2v5z 2v60 2v61 2vrl 2vrm 2vz2 2xcg 2xfn 2xfo 2xfp 2xfq } \\
\text { 2xfu 3po7 3zyx 4a79 4a7a 4crt } 5 \mathrm{mrl}\end{array}$ \\
\hline ADAM 17/ Peptidase/ T82393 & $\begin{array}{l}\text { 1bkc } 1 \text { zxc } 2 \mathrm{a} 8 \mathrm{~h} 2 \mathrm{ddf} 2 \mathrm{fv} 52 \mathrm{fv} 92 \mathrm{i} 472 \mathrm{~m} 2 \mathrm{f} 2 \mathrm{oi} 03 \mathrm{~b} 92 \text { 3cki 3e8r 3edz 3ewj } 3 \mathrm{~g} 423 \mathrm{kmc} 3 \mathrm{kme} \\
310 \mathrm{t} 310 \mathrm{v} 3 \mathrm{le} 93 \text { lea } 3 \operatorname{lgp} 3 \mathrm{o} 64\end{array}$ \\
\hline $\begin{array}{l}\text { Estrogen receptor beta/ Nuclear } \\
\text { hormone receptor/ T } 80896\end{array}$ & $\begin{array}{l}\text { 1l2j 1nde 1qkm 1u3q 1u3r 1u3s 1u9e 1x76 1x78 1x7b 1x7j 1yy4 1yye 1zaf 2fsz 2giu 2i0g } \\
2 \text { jj3 2nv7 2qtu 2yjd 2yly 2z4b 3oll 3ols 3omo 3omp 3omq 4j24 4j26 4zi1 5toa }\end{array}$ \\
\hline PI3K alpha/ Kinase/ T80276 & 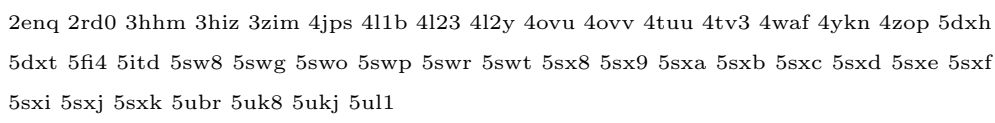 \\
\hline $\begin{array}{l}\text { Glycogen synthase kinase- } 3 \text { beta/ } \\
\text { Kinase/ T70977 }\end{array}$ & $\begin{array}{l}\text { 1gng 1h8f 1i09 1j1b 1j1c 1o6k 1o6l 1o9u 1pyx 1q3d 1q3w 1q41 1q4l 1q5k 1r0e 1uv5 2jdo } \\
\text { 2jdr 2jld 2o5k 2ow3 2uw9 2x39 2xh5 3cqu 3cqw 3du8 3e87 3e88 3e8d 3f7z 3f88 3gb2 3i4b } \\
\text { 311s 3m1s 3mv5 3ow4 3pup 3q3b 3qkk 3say 3sd0 3zdi 3zrk 3zrl 3zrm 4acc 4acd 4acg 4ach } \\
\text { 4afj 4b7t 4dit 4ekk 4iq6 4j1r 4j71 4nm0 4nm3 4nm5 4nm7 4ptc 4pte 4ptg 5f94 5f95 5hln } \\
\text { 5hlp 5k5n 5oy4 6b8j }\end{array}$ \\
\hline
\end{tabular}


Cell division protein kinase 2/ Kinase/ T70176 1aq1 1b38 1b39 1buh 1ckp 1di8 1dm2 1e1v 1e1x 1e9h 1f5q 1fin 1fq1 1fvt 1fvv 1g5s 1gih 1gii 1gij 1gy3 1gz8 1h00 1h01 1h07 1h08 1h0v 1h0w 1h1p 1h1q 1h1r 1h1s 1h24 1h25 1h26 1h27 1h28 1hck 1hcl 1jst 1jsu 1jsv 1jvp 1ke5 1ke6 1 ke7 1 ke8 1 ke9 1 ogu 1 oi9 1 oiq 1 oir 1oit 1oiu 1oiy 1okv 1okw 1ol1 1ol2 1p2a 1p5e 1pf8 1pkd 1pw2 1pxi 1pxj 1pxk 1pxl 1pxm 1 pxn 1pxo 1pxp 1pye 1qmz 1r78 1urc 1urw 1v1k 1vyw 1vyz 1w0x 1w8c 1w98 1wcc 1y8y 1y91 1ykr 2a0c 2a4l 2b52 2b53 2b54 2b55 2bhe 2bhh 2bkz 2bpm 2btr 2bts 2c4g 2c5n 2c5o 2c5v 2c5x 2c5y 2c68 2c69 2c6i 2c6k 2c61 2c6m 2c6o 2c6t 2cch 2cci 2cjm 2clx 2ds1 2duv 2exm 2fvd 2g9x 2hic 2i40 2iw6 2iw8 2iw9 2j9m 2jgz 2r3f 2r3g 2r3h 2r3i 2r3j 2r3k 2r31 2r3m 2r3n 2r3o 2r3p 2r3q 2r3r 2r64 2uue 2uzb 2uzd 2uze 2uzl 2uzn 2uzo 2v0d 2v22 2vta 2vth 2vti 2vtj 2vtl 2vtm 2vtn 2vto 2vtp 2vtq 2vtr 2vts 2vtt 2vu3 2vv9 2w05 2w06 2w17 2 w 1 h 2 wev 2 wfy 2 whb 2 wih 2 wip 2 wma 2 wmb 2 wpa 2 wxv $2 x 1$ n 2 xmy $2 x n b 3$ bht 3 bhu 3bhv 3ddp 3ddq 3dog 3eid 3ej1 3eoc 3ezr 3ezv 3f5x 3fz1 3ig7 3igg 3le6 3lfn 3lfq 3lfs 3my5 3ns9 3pj8 3pxf 3pxq 3pxr 3pxy 3pxz 3py0 3py1 3qhr 3qhw 3ql8 3qqf 3qqg 3qqh 3qqj 3qqk 3qql 3qrt 3qru 3qtq 3qtr 3qts 3qtu 3qtw 3qtx 3qtz 3qu0 3qwj 3qwk 3qx2 3qx4 3qxo 3qxp 3qzf 3qzg 3qzh 3qzi 3r1q 3r1s 3r1y 3r28 3r6x 3r71 3r73 3r7e 3r7i 3r7u 3r7v 3r7y 3r83 3r81 3r8m 3r8p 3r8u 3r8v 3r8z 3r9d 3r9h 3r9n 3r9o 3rah 3rai 3rak 3ral 3rjc 3rk5 3rk7 3rk9 3rkb 3rm6 3rm7 3rmf 3rni 3roy 3rpo 3rpr 3rpv 3rpy 3rzb 3s00 3s0o 3s1h 3s2p 3sqq 3sw 4 3sw7 3ti1 3tiy 3tiz 3tnw 3uli 3unj 3unk 3wbl 4acm 4bck 4bcm 4bcn 4bco 4bcp 4bcq 4bgh $4 \mathrm{bzd} 4 \mathrm{cfm} 4 \mathrm{cfn} 4 \mathrm{cfu} 4 \mathrm{cfv} 4 \mathrm{cfw} 4 \mathrm{cfx} 4 \mathrm{~d} 1 \mathrm{x} 4 \mathrm{~d} 1 \mathrm{z} 4 \mathrm{ek} 3$ 4ek4 4ek5 4ek6 4ek8 4eoi 4eoj 4eok 4 eol 4 eom 4eon 4eoo 4eop 4eoq 4eor 4eos 4erw 4ez3 4ez7 4fkg 4fki 4fkj 4fkl 4fko 4fkp 4fkq $4 \mathrm{fkr} 4 \mathrm{fks} 4 \mathrm{fkt} 4 \mathrm{fku} 4 \mathrm{fkv} 4 \mathrm{fkw} 4 \mathrm{fx} 3$ 4gcj 4i3z 4ii5 4kd1 4lyn 4nj3 4rj3 5a14 5and 5ane 5ang 5 ani 5 anj 5 ank 5 ano 5 cyi 5d1j 5fp5 5fp6 5iev 5iex 5iey 5if1 5jq5 5jq8 5k4j 5l2w 5lmk 5nev 5uq1 5uq2 5uq3

4fyq 4 fyr 4 fys 4 fyt 5 lhd

Aminopeptidase N/ Peptidase/ T67272

Vascular endothelial growth factor receptor 1/ Kinase/ T63966

Histone deacetylase 4/ Carbonnitrogen hydrolase/ T63816

Proto-oncogene c-Abl/ Kinase/
T63505

1ab2 1abl 1awo 1bbz 1ju5 1opl 1zzp 2abl 2e2b 2f4j 2fo0 2g1t 2g2f 2g2h 2g2i 2gqg 2hiw 2hyy 2 hz0 2hz4 2hzi 2 o88 2v7a 3cs9 3eg0 3eg1 3eg2 3eg3 3egu 3k2m 3pyy 3qri 3qrj 3qrk 3t04 3ue4 3uyo 4j9b 4j9c 4j9d 4j9e 4j9f 4j9g 4j9h 4j9i 4jjb 4jjc 4jjd 4twp 4wa9 4xey 4yc8 4 zog 5 dc0 5dc4 5dc9 5hu9 5mo4 5oaz 6amv 6amw

Tyrosine-protein kinase SYK/ Kinase/ T62431

1 a 81 1csy $1 \mathrm{csz} 1 \mathrm{xba} 1 \mathrm{xbb} 1 \mathrm{xbc} 3 \mathrm{buw} 3 \mathrm{emg} 3 \mathrm{fqe} 3 \mathrm{fqh} 3 \mathrm{fqs} 3 \mathrm{srv} 3 \mathrm{tub} 3$ tuc 3tud 3vf8 3vf9 4dfl 4dfn 4f4p 4fl1 4fl2 4fl3 4fyn 4fyo 4fz6 4fz7 4gfg 4i0r 4i0s 4i0t 4puz 4pv0 4px6 4rss 4rx7 4rx8 4rx9 4wnm 4xg2 4xg3 4xg4 4xg6 4xg7 4xg8 4xg9 4yjo 4yjp 4yjq 4yjr 4yjs 4yjt 4 yju 4yjv 5c26 5c27 5cxh 5cxz 5cy3 5ghv 5lma 5lmb 5t68 5tiu 5tr6 5tt7

Antiapoptotic protein BCL-XL/ Bcl-2 family/ T56510

1bxl 1g5j 1lxl 1maz 1r2d 1r2e 1r2g 1r2h 1r2i 1ysg 1ysi 1ysn 2b48 2lp8 2lpc 2m03 2m04 2me8 2me9 2mej 2o1y 2o2m 2o2n 2p1l 2pon 2yj1 2yq6 2yq7 2yxj 3cva 3fdl 3fdm 3inq 3io8 3pl7 3qkd 3r85 3sp7 3spf 3wiz 3zk6 3zln 3zlo 3zlr 4a1u 4a1w 4aq3 4bpk 4c52 4c5d 4cin 4ehr 4hnj 4ieh 4ppi 4qve 4qvf 4qvx 4tuh 4z9v 5agw 5agx 5b1z 5c3g 5fmj 5fmk 6bf2 


\begin{tabular}{|c|c|}
\hline $\begin{array}{l}\text { Lanosterol synthase/ Intramolecu- } \\
\text { lar transferases/ T56175 }\end{array}$ & $1 \mathrm{w} 6 \mathrm{j} 1 \mathrm{w} 6 \mathrm{k}$ \\
\hline $\begin{array}{l}\text { TGF-beta receptor type I/ Kinase/ } \\
\text { T53389 }\end{array}$ & 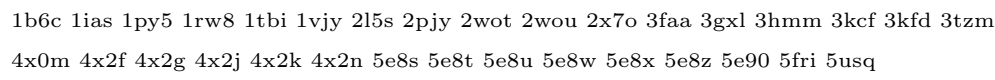 \\
\hline $\begin{array}{l}\text { Nociceptin receptor/ GPCR } \\
\text { rhodopsin/ T52921 }\end{array}$ & 4 ea3 5 dhg $5 \mathrm{dhh}$ \\
\hline $\begin{array}{l}\text { Insulin-like growth factor I recep- } \\
\text { tor/ Kinase/ T48069 }\end{array}$ & $\begin{array}{l}\text { 1igr 1jqh 1k3a } 1 \mathrm{~m} 7 \mathrm{n} \text { 1p4o } 2 \text { oj9 } 2 \mathrm{zm} 3 \text { 3d94 3f5p 3i81 3lvp 3lw0 3nw5 3nw6 3nw7 3o23 } \\
3 \mathrm{qqu} 4 \mathrm{~d} 2 \mathrm{r} 4 \mathrm{xss} 5 \text { fxq } 5 \text { fxr } 5 \text { fxs } 5 \mathrm{hzn}\end{array}$ \\
\hline $\begin{array}{l}\text { Hepatocyte growth factor receptor/ } \\
\text { Kinase/ T40474 }\end{array}$ & 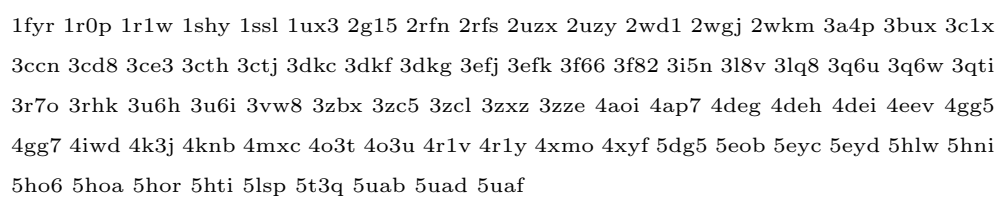 \\
\hline $\begin{array}{l}\text { Protein kinase C, beta type/ Ki- } \\
\text { nase/ T40276 }\end{array}$ & $2 \mathrm{iOe}$ \\
\hline $\begin{array}{l}\text { Mitogen-activated protein kinase 8/ } \\
\text { Kinase/ T40097 }\end{array}$ & $\begin{array}{l}\text { 1ukh 1uki 2g01 2gmx 2h96 2no3 2xrw 2xs0 3elj 3o17 3o2m 3pze 3v3v 3vud 3vug 3vuh } \\
\text { 3vui 3vuk 3vul 3vum 4awi 4e73 4g1w 4hys 4hyu 4izy 4lff 4qtd 4ux9 4yr8 5len }\end{array}$ \\
\hline $\begin{array}{l}\text { Calmodulin/ Calmodulin- } \\
\text { dependent secretion/ T39610 }\end{array}$ & 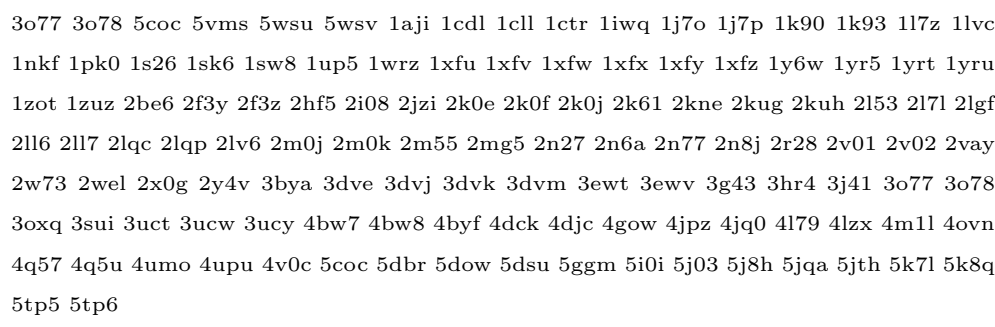 \\
\hline $\begin{array}{l}\text { Peroxisomeproliferator activated } \\
\text { receptor delta/ Zinc-finger/ T36557 }\end{array}$ & $\begin{array}{l}\text { 1gwx 1y0s 2awh 2b50 2baw 2env 2gwx 2j14 2q5g 2xyj 2xyw 2xyx 2znp 2znq 3d5f 3dy6 } \\
\text { 3et2 3gwx 3gz9 3oz0 3peq 3sp9 3tkm 5u3q 5u3r 5u3s 5u3t 5u3u 5u3v 5u3w 5u3x 5u3y } \\
5 \mathrm{u} 3 \mathrm{z} 5 \mathrm{u} 405 \mathrm{u} 415 \mathrm{u} 425 \mathrm{u} 435 \mathrm{u} 445 \mathrm{u} 455 \mathrm{u} 46\end{array}$ \\
\hline $\begin{array}{l}\text { Thrombin receptor/ GPCR } \\
\text { rhodopsin/ T36483 }\end{array}$ & 1nrn 1 nro $1 \mathrm{nrp} 1 \mathrm{nrq} 1 \mathrm{nrr} 3$ bef $3 \mathrm{hki} 3 \mathrm{hkj} 3 \mathrm{lu} 9$ svw 7 \\
\hline $\begin{array}{l}\text { Dual specificity mitogen-activated } \\
\text { protein kinase kinase } 1 / \text { Kinase/ } \\
\text { T35940 }\end{array}$ & $\begin{array}{l}\text { 1s9j 2p55 3dv3 3dy7 3e8n 3eqb 3eqc 3eqd 3eqf 3eqg 3eqh 3eqi 3mbl 3orn 3os3 3pp1 3sls } \\
\text { 3v01 3v04 3vvh 3w8q 3wig 3zls 3zlw 3zlx 3zly 3zm4 4an2 4an3 4an9 4anb 4ark 4lmn 4mne } \\
4 \mathrm{u} 7 \mathrm{z} 4 \mathrm{u} 804 \mathrm{u} 815 \mathrm{bx} 05 \text { eym 5hze }\end{array}$ \\
\hline $\begin{array}{l}\text { Lymphocyte function-associated } \\
\text { antigen 1/ T35640 }\end{array}$ & 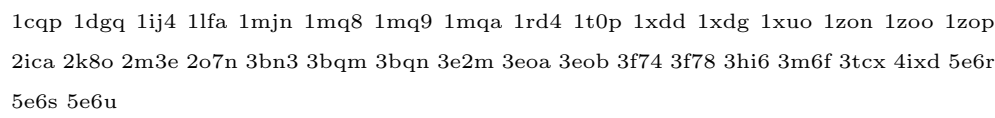 \\
\hline
\end{tabular}




\begin{tabular}{|c|c|}
\hline Collagenase 3/ Peptidase/ T34296 & $\begin{array}{l}\text { 1eub 1fls } 1 \text { fm1 1pex 1uc1 1xuc 1xud 1xur 1you 1ztq 2d1n 2e2d 2ow9 2ozr 2pjt 2yig 3elm } \\
3 \text { i7g 3i7i 3kec 3kej 3kek 3kry 3ljz 3o2x 3tvc 3wv1 3wv2 3wv3 3zxh 456c 4a7b 4fu4 4fvl } \\
4 \text { g0d 4jp4 4jpa } 4119 \text { 5b5o 5b5p 5bot 5boy 5bpa 5uwk 5uwl 5uwm 5uwn 830c }\end{array}$ \\
\hline Integrin alpha-5/ Integrin/ T29879 & 3vi3 3vi4 4wjk 4wk0 4wk2 4wk4 \\
\hline $\begin{array}{l}\text { Metabotropicglutamate receptor } 1 / \\
\text { GPCR glutamate/ T2 } 27137\end{array}$ & $3 \mathrm{ks} 94$ or 2 \\
\hline $\begin{array}{l}\text { Tyrosine-protein kinase JAK3/ Ki- } \\
\text { nase/ T23172 }\end{array}$ & $\begin{array}{l}\text { 1yvj 3lxk 3lxl 3pjc 3zc6 3zep 4hvd 4hvg 4hvh 4hvi 4i6q 4qps 4qt1 4rio 4v0g 4z16 5lwm } \\
\text { 5lwn 5toz 5tts 5ttu 5ttv 5vo6 5w86 5wfj }\end{array}$ \\
\hline $\begin{array}{l}\text { NAD-dependent deacetylase } \\
\text { sirtuin-1/ Carbon-nitrogen hy- } \\
\text { drolase/ T14731 }\end{array}$ & 4i5i 4 if6 4 ig9 $4 \mathrm{kxq} 4 \mathrm{zzh}$ 4zzi $4 \mathrm{zzj} 5 \mathrm{btr}$ \\
\hline $\begin{array}{l}\text { Sphingosine 1-phosphate receptor } \\
1 / \text { GPCR rhodopsin/ T13852 }\end{array}$ & $3 v 2 w 3 v 2 y$ \\
\hline $\begin{array}{l}\text { Retinoic acid receptor RXR-alpha/ } \\
\text { Zinc-finger/ T13726 }\end{array}$ & 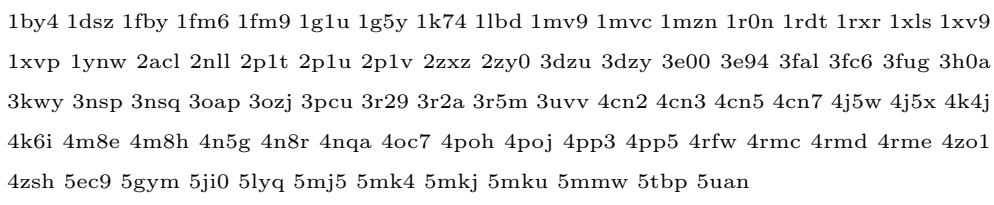 \\
\hline $\begin{array}{l}\text { Carbonic anhydrase I/ Carbon- } \\
\text { oxygen lyases/ T13201 }\end{array}$ & $\begin{array}{l}\text { 1azm } 1 \mathrm{bzm} 1 \mathrm{crm} 1 \mathrm{czm} 1 \mathrm{hcb} 1 \mathrm{hug} 1 \mathrm{huh} 1 \mathrm{j} 9 \mathrm{w} \text { 1jv0 2cab 2foy } 2 \mathrm{fw} 42 \mathrm{it} 42 \mathrm{nmx} 2 \mathrm{nn} 12 \mathrm{nn} 7 \\
\text { 3lxe } 3 \mathrm{w} 6 \mathrm{~h} 3 \mathrm{w} 6 \mathrm{i} 4 \mathrm{wr} 74 \mathrm{wup} 4 \mathrm{wuq} 5 \mathrm{e} 2 \mathrm{~m} 5 \mathrm{gmm}\end{array}$ \\
\hline
\end{tabular}




\begin{tabular}{|c|c|}
\hline $\begin{array}{l}\text { Acetyl-CoA carboxylase } 2 / \mathrm{Lig}- \\
\text { ases forming carbon-carbon bonds/ } \\
\text { T08922 }\end{array}$ & $2 \mathrm{dn} 82 \mathrm{hjw} 2 \mathrm{kcc} 3 \mathrm{ff} 63$ gid $3 \mathrm{glk} 3 \mathrm{jrw} 3 \mathrm{jrx} 3 \mathrm{tdc} 4 \mathrm{hq} 65 \mathrm{kkn}$ \\
\hline mRNA of PDK-1/ Kinase/ T08074 & $\begin{array}{l}\text { 1h1w 1oky 1okz 1uu3 1uu7 1uu8 1uu9 1uvr 1w1d 1w1g 1w1h 1z5m 2biy 2pe0 2pe1 2pe2 } \\
\text { 2r7b 2vki 2xch 2xck 3h9o 3hrc 3hrf 3ion 3iop 3nax 3nay 3nun 3nus 3nuu 3nuy 3orx 3orz } \\
\text { 3otu 3pwy 3qc4 3qcq 3qcs 3qcx 3qcy 3qd0 3qd3 3qd4 3rcj 3rwp 3rwq 3sc1 4a06 4a07 4aw0 } \\
\text { 4aw1 4ct1 4ct2 4rqk 4rqv 4rrv 4xx9 5ack 5hkm 5hng 5ho7 5ho8 5lvl 5lvm 5lvn 5lvo 5lvp } \\
\text { 5mrd }\end{array}$ \\
\hline $\begin{array}{l}\text { CGMP-specific } 3^{\prime}, 5^{\prime} \text {-cyclic phos- } \\
\text { phodiesterase/ Phosphoric diester } \\
\text { hydrolases/ T07663 }\end{array}$ & $\begin{array}{l}\text { 1rkp 1t9r 1t9s 1tbf 1udt 1udu 1uho 1xoz 1xp0 2chm 2h40 2h42 2h44 2xss 3b2r 3bjc 3hc8 } \\
\text { 3hdz 3jwq 3jwr 3lfv 3mfo 3shy 3shz 3sie 3tge 3tgg 4g2w 4g2y 4i9z 4ia0 4md6 4oew 4oex } \\
\text { 5jo3 }\end{array}$ \\
\hline $\begin{array}{l}\text { Neurotrophic tyrosine kinase recep- } \\
\text { tor } 1 / \text { Kinase/ T07173 }\end{array}$ & $\begin{array}{l}\text { 1he7 1shc } 1 \text { wwa } 1 \text { www } 2 \text { ifg } 2 \mathrm{n} 90 \text { aoj } 4 \mathrm{crp} 4 \text { foi } 4 \mathrm{gt} 54 \mathrm{pmm} 4 \mathrm{pmp} 4 \mathrm{pms} 4 \mathrm{pmt} 4 \mathrm{yne} 4 \mathrm{yps} \\
5 \mathrm{~h} 3 \mathrm{q} 5 \mathrm{i} 8 \mathrm{a} 5 \mathrm{jfs} 5 \mathrm{jfv} 5 \mathrm{jfw} 5 \mathrm{jfx} 5 \mathrm{kmi} 5 \mathrm{kmj} 5 \mathrm{kmk} 5 \mathrm{kml} 5 \mathrm{kmm} 5 \mathrm{kmn} 5 \mathrm{kmo}\end{array}$ \\
\hline $\begin{array}{l}\text { Poly [ADP-ribose] polymerase-1/ } \\
\text { Pentosyltransferase/ T06273 }\end{array}$ & 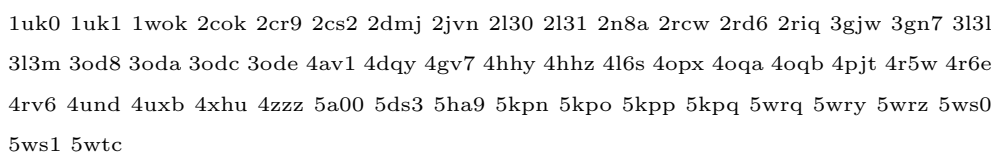 \\
\hline $\begin{array}{l}\text { Nitric oxide synthase, inducible/ } \\
\text { Oxidoreductases acting on paired } \\
\text { donors/ T02703 }\end{array}$ & 1nsi 2116 2nsi $3 \mathrm{e} 7 \mathrm{~g} 3 \mathrm{ej} 83 \mathrm{hr} 4$ 4cx7 4nos 5 tp6 $5 \mathrm{xn} 3$ \\
\hline $\begin{array}{l}\mathrm{D}(3) \text { dopamine receptor/ GPCR } \\
\text { rhodopsin/ T02551 }\end{array}$ & $3 \mathrm{pbl}$ \\
\hline $\begin{array}{l}\text { Phosphoinositide } 3 \text { kinase/ Kinase/ } \\
\text { T95913 }\end{array}$ & 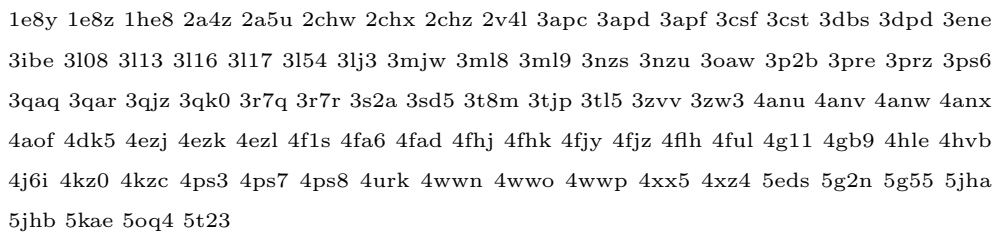 \\
\hline $\begin{array}{l}\text { Ribosomal protein S6 kinase/ Ki- } \\
\text { nase/ T94479 }\end{array}$ & 3a60 3a61 3a62 3we4 3wf5 3wf6 3wf7 3wf8 3wf9 413j 4l31 41424143414441454146 4rlo 4rlp \\
\hline $\begin{array}{l}\text { Receptor protein-tyrosine kinase } \\
\text { erbB-4/ Kinase/ T92057 }\end{array}$ & 2 ahx 212 t 2 lcx 2 r 4 b 3bbt 3 bbw 3 bce $3 u 2 p ~ 3 u 7 u ~ 3 u 9 u$ \\
\hline mRNA of B-Raf/ Kinase/ T90648 & 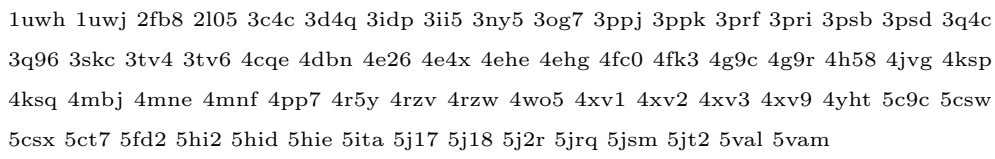 \\
\hline
\end{tabular}




\begin{tabular}{|c|c|}
\hline $\begin{array}{l}\text { Glycinamide ribonucleotide formyl- } \\
\text { transferase/ Carbon-nitrogen lig- } \\
\text { ase/ T89918 }\end{array}$ & 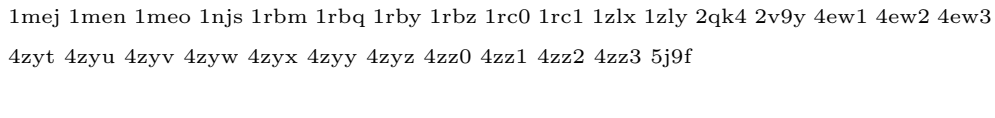 \\
\hline $\begin{array}{l}17 \text { alpha-hydroxylase-C17, } 20- \\
\text { lyase/ Oxidoreductases acting on } \\
\text { paired donors/ T89041 }\end{array}$ & 2c17 3ruk 3swz 4nkv 4nkw 4nkx 4nky 4nkz 5irq 5irv \\
\hline $\begin{array}{l}\text { Protein farnesyltransferase beta } \\
\text { subunit/ Alkyl aryl transferase/ } \\
\text { T86428 }\end{array}$ & 1jcq $1 \mathrm{ld} 7$ 1ld 8 1mzc $1 \mathrm{~s} 63$ 1sa 4 tn6 2f0y $2 \mathrm{~h} 6 \mathrm{f} 2 \mathrm{~h} 6 \mathrm{~g} 2 \mathrm{~h} 6 \mathrm{~h} 2 \mathrm{~h} 6$ i 2 iej $3 \mathrm{e} 37$ \\
\hline $\begin{array}{l}\text { Coagulation factor IX/ Peptidase/ } \\
\text { T83369 }\end{array}$ & $\begin{array}{l}\text { 1cfh } 1 \mathrm{cfi} 1 \mathrm{edm} 1 \mathrm{ixa} 1 \mathrm{mgx} 1 \mathrm{nl0} 1 \mathrm{rfn} 2 \mathrm{wph} 2 \mathrm{wpi} 2 \mathrm{wpj} 2 \mathrm{wpk} 2 \mathrm{wpl} 2 \mathrm{wpm} 3 \mathrm{kcg} 3 \mathrm{lc} 33 \mathrm{lc} 5 \\
4 \mathrm{wm} 04 \mathrm{wma} 4 \mathrm{wmb} 4 \mathrm{wmi} 4 \mathrm{wmk} 4 \mathrm{wn} 24 \mathrm{wnh} 4 \mathrm{yzu} 4 \mathrm{z} 0 \mathrm{k} 4 \mathrm{zae} 5 \mathrm{egm} 5 \mathrm{f} 845 \mathrm{f} 855 \mathrm{f} 865 \mathrm{jb} 85 \mathrm{jb} 9 \\
5 \mathrm{jba} 5 \mathrm{jbb} 5 \mathrm{jbc} 5 \text { tno } 5 \mathrm{tnt} 5 \mathrm{vyg}\end{array}$ \\
\hline $\begin{array}{l}\text { Angiotensin-converting enzyme/ } \\
\text { Glycosylases/ T } 82577\end{array}$ & $\begin{array}{l}\text { 1o86 1o8a 1uze 1uzf 2c6f 2c6n 2iul 2iux 2oc2 2xy9 2xyd 2ydm 3bkk 3bkl 313n 3nxq 4aph } \\
4 \text { apj } 4 \text { bxk } 4 \text { bzr } 4 \text { bzs } 4 \mathrm{c} 2 \mathrm{n} 4 \mathrm{c} 2 \mathrm{o} 4 \mathrm{c} 2 \mathrm{p} 4 \mathrm{c} 2 \mathrm{q} 4 \mathrm{c} 2 \mathrm{r} 4 \mathrm{ca} 5 \text { 4ca6 } 4 \text { ufa } 4 \text { ufb } 5 \mathrm{am} 85 \mathrm{am} 95 \mathrm{ama} \\
5 \mathrm{amb} 5 \mathrm{amc}\end{array}$ \\
\hline $\begin{array}{l}\text { Retinoic acid receptor gamma/ } \mathrm{Nu}- \\
\text { clear hormone receptor/ } \mathrm{T} 82146\end{array}$ & 1exa 1 exx 1 fcx 1 fcy 1 fcz 1 fdo 2 lbd 3 lbd $41 \mathrm{bd} 5 \mathrm{~m} 24$ \\
\hline $\begin{array}{l}\text { mRNA of Inhibitor of apoptosis } \\
\text { protein/ Target of antisense drug/ } \\
\text { T81892 }\end{array}$ & 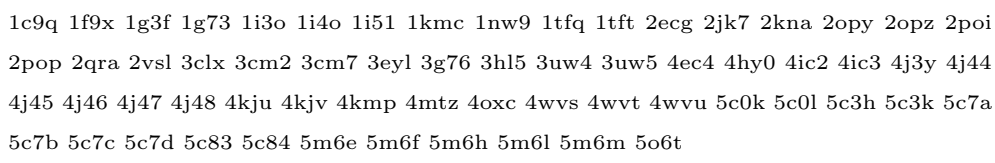 \\
\hline $\begin{array}{l}\text { Squalene synthetase/ Alkyl aryl } \\
\text { transferase/ T } 81850\end{array}$ & $\begin{array}{l}\text { 1ezf 3asx 3lee 3q2z 3q30 3v66 3vj8 3vj9 3vja 3vjb 3vjc 3wc9 3wcd 3wcf 3wch 3wci 3wcj } \\
3 w c l \text { 3wcm 3wef 3weg 3weh 3wei 3wej 3wek 3wsa }\end{array}$ \\
\hline MAP kinase p38/ Kinase/ T79798 & $1 \mathrm{~cm} 84 \mathrm{qum}$ \\
\hline $\begin{array}{l}\text { Thyroid hormone receptor alpha/ } \\
\text { Nuclear hormone receptor/ T79591 }\end{array}$ & 1nav 2 h77 2 h79 3 hzf 3 ilz 3 jzb $4 \operatorname{lnw} 4 \ln x$ \\
\hline $\begin{array}{l}\text { Catechol-O-methyl-transferase/ } \\
\text { Methyltransferase superfamily/ } \\
\text { T76904 }\end{array}$ & 3a7e 3bwm 3bwy 4pyi 4pyj 4pyk 4xuc 4xud 4xue 5lsa \\
\hline $\begin{array}{l}\text { Antithrombin-III/ Serpin family/ } \\
\text { T73476 }\end{array}$ & 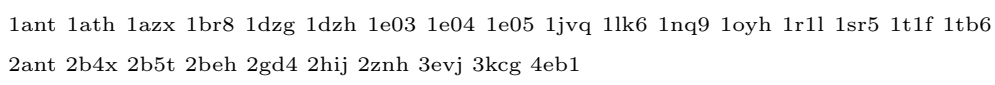 \\
\hline $\begin{array}{l}\text { Tissue factor/ Transmembrane pro- } \\
\text { tein/ T72702 }\end{array}$ & $\begin{array}{l}\text { 1ahw 1boy 1dan 1fak 1j9c 1jps 1nl8 1o5d 1tfh 1uj3 1w0y 1w2k 1wqv 1wss 1wtg 1wun } \\
1 \text { wv7 1z6j 2a2q 2aei 2aer 2b7d 2b8o 2c4f 2cef 2ceh 2cez 2cfj 2ec9 2f9b 2fir 2flb 2flr 2hft } \\
\text { 2puq 2zp0 2zwl 2zzu 3ela 3th2 3th3 3th4 4ibl 4m7l 4ylq 4z6a 4zma 5w06 }\end{array}$ \\
\hline
\end{tabular}




\begin{tabular}{|c|c|}
\hline $\begin{array}{l}\text { Mineralocorticoid receptor/ Zinc- } \\
\text { finger/ T72168 }\end{array}$ & 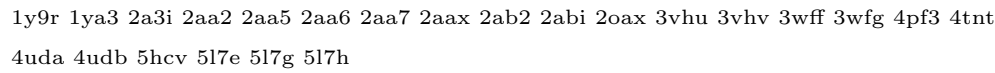 \\
\hline $\begin{array}{l}\text { Corticosteroid } 11 \text {-beta- } \\
\text { dehydrogenase, isozyme } 1 / \text { Short- } \\
\text { chain dehydrogenases reductases/ } \\
\text { T65200 }\end{array}$ & $\begin{array}{l}\text { 1xu7 1xu9 2bel 2ilt 2irw 2rbe 3byz 3bzu 3ch6 3czr 3d3e 3d4n 3d5q 3ey4 3fco 3frj 3h6k } \\
3 \text { hfg 3oq1 3pdj 3qqp 3tfq 4bb5 4bb6 4c7j 4c7k 4hfr 4hx5 4iju 4ijv 4ijw 4k1l 4p38 4yyz } \\
5 \text { pgu 5pgv 5pgw 5pgx 5pgy }\end{array}$ \\
\hline JAK1/ Kinase/ T62460 & 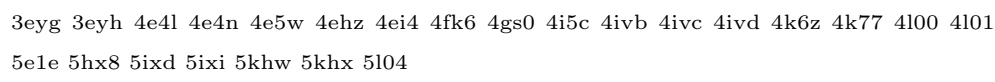 \\
\hline $\begin{array}{l}\text { Interleukin-2/ Cytokine: inter- } \\
\text { leukin/ T61698 }\end{array}$ & $\begin{array}{l}\text { 1ilm 1iln 1irl 1m47 1m48 1m49 1m4a 1m4b 1m4c 1nbp 1pw6 1py2 1qvn 1z92 2b5i 2erj } \\
3 \text { ink 3qaz 3qb1 4nej 4nem 5lqb 5m5e }\end{array}$ \\
\hline $\begin{array}{l}\text { Kappa-type opioid receptor/ GPCR } \\
\text { rhodopsin/ T60693 }\end{array}$ & $2 \mathrm{aOd} 2 \mathrm{iqn} 4 \mathrm{djh}$ \\
\hline $\begin{array}{l}\text { Mast/ stem cell growth factor re- } \\
\text { ceptor/ T57700 }\end{array}$ & 1pkg 1qzj 1qzk 1r01 1t45 1t46 2e9w 2ec8 2iuh 2vif 3g0e 3g0f 4hvs $4 \mathrm{k} 94$ 4k9e 4pgz 4u0i \\
\hline $\begin{array}{l}\text { S-adenosylmethioninedecarboxylase } \\
\text { proenzyme/ Carbon-carbon lyase/ } \\
\text { T55922 }\end{array}$ & $\begin{array}{l}\text { 1i72 1i79 1i7b 1i7c } 1 \mathrm{i} 7 \mathrm{~m} 1 \text { jen } 1 \mathrm{j} 10 \text { 1msv 3dz2 3dz3 3dz4 3dz5 3dz6 3dz7 3ep3 3ep4 3ep5 } \\
\text { 3ep6 3ep7 3ep8 3ep9 3epa 3epb 3h0v 3h0w }\end{array}$ \\
\hline $\begin{array}{l}\text { Mitogen-activated protein kinase } \\
11 / \text { Kinase/ T55729 }\end{array}$ & $3 \operatorname{gc} 83 g c 93 \operatorname{gp} 0$ \\
\hline $\begin{array}{l}\text { cystic fibrosis transmembrane con- } \\
\text { ductance regulator/ Acid anhy- } \\
\text { drides hydrolase/ T55654 }\end{array}$ & 1nbd 1 xmi 1 xmj 2 bbo 2 bbs 2 bbt 2 lob 2 pze 2 pzf 2 pzg $3 \mathrm{gd} 73$ isw 4 wz 6 5d2d 5 d3e 5 d $3 f$ fuak \\
\hline $\begin{array}{l}\text { Nicotinamide } \quad \text { phosphoribosyl- } \\
\text { transferase/ Pentosyltransferase/ } \\
\text { T54582 }\end{array}$ & $\begin{array}{l}\text { 2e5b 2e5c 2e5d 2gvg 2gvj 3dgr 3dhd 3dhf 3dkj 3dkl 4jnm 4jr5 4kfn 4kfo 4kfp 4l41 4l4m } \\
\text { 4lts 4lv9 4lva 4lvb 4lvd 4lvf 4lvg 4lww 4m6p 4m6q 4n9b 4n9c 4n9d 4n9e 4o0z 4o10 4o12 } \\
\text { 4o13 4o14 4o15 4o16 4o17 4o18 4o19 4o1a 4o1b 4o1c 4o1d 4o28 4wq6 5kit 5lx3 5lx5 5u2m } \\
\text { 5u2n 5upe 5upf }\end{array}$ \\
\hline $\begin{array}{l}\text { Carbonic anhydrase IV/ Carbon- } \\
\text { oxygen lyases/ T53378 }\end{array}$ & $1 \mathrm{znc} 3 \mathrm{f} 7 \mathrm{~b} 3 \mathrm{f} 7 \mathrm{u} 3 \mathrm{fw} 35 \mathrm{ipz} 5 \mathrm{jn} 85 \mathrm{jn} 95 \mathrm{jna} 5 \mathrm{jnc} 5 \mathrm{ku} 6$ \\
\hline $\begin{array}{l}\text { NAD }(\mathrm{P}) \mathrm{H} \text { dehydrogenase [quinone] } \\
1 / \text { Oxidoreductases acting on } \\
\text { NADH or NADPH/ T52389 }\end{array}$ & 1d4a 1dxo 1gg5 1h66 1h69 1kbo 1kbq 1qbg 2f1o 3jsx 4cet 4cf6 5a4k 5ea2 5eai 5 fuq \\
\hline
\end{tabular}




\begin{tabular}{|c|c|}
\hline $\begin{array}{l}\text { Casein kinase II, alpha chain/ Ki- } \\
\text { nase/ T51565 }\end{array}$ & 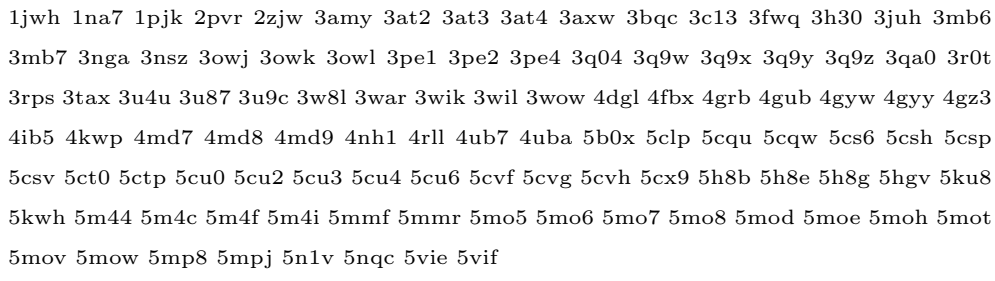 \\
\hline $\begin{array}{l}\text { Toll-like receptor } 8 / \text { Toll-like recep- } \\
\text { tor family/ T48703 }\end{array}$ & $\begin{array}{l}\text { 3w3g 3w3j 3w3k 3w31 3w3m 3w3n 3wn4 4qbz 4qc0 4r07 4r08 4r09 4r0a 4r6a 5awa 5awb } \\
5 \text { awc 5awd 5az5 5hdh }\end{array}$ \\
\hline $\begin{array}{l}\text { mRNA of androgen receptor/ Tar- } \\
\text { get of antisense drug/ T46521 }\end{array}$ & $\begin{array}{l}\text { 1e3g } 1 \text { gs4 } 1 \text { t5z } 1 \text { t63 } 1 \text { t65 1xj7 1xow 1xq3 1z95 2am9 2ama 2amb 2ao6 2ax6 2ax7 2ax8 } \\
\text { 2ax9 2axa 2hvc 2oz7 2pio 2pip 2piq 2pir 2pit 2piu 2piv 2piw 2pix 2pkl 2pnu 2q7i 2q7j } \\
\text { 2q7k 2q7l 2yhd 2ylo 2ylp 2ylq 2z4j 3b5r 3b65 3b66 3b67 3b68 3btr 313x 313z 3rlj 3rll 3v49 } \\
\text { 3v4a 3zqt 4hlw 4k7a 4oea 4oed 4oey 4oez 4ofr 4ofu 4ogh 4oh5 4oh6 4oha 4oil 4oiu 4oj9 } \\
\text { 4ojb 4ok1 4okb 4okt 4okw 4okx 4olm 4ql8 5cj6 5jjm 5t8e 5t8j 5v8q }\end{array}$ \\
\hline $\begin{array}{l}\text { Coagulation factor VII/ Peptidase/ } \\
\text { T43332 }\end{array}$ & 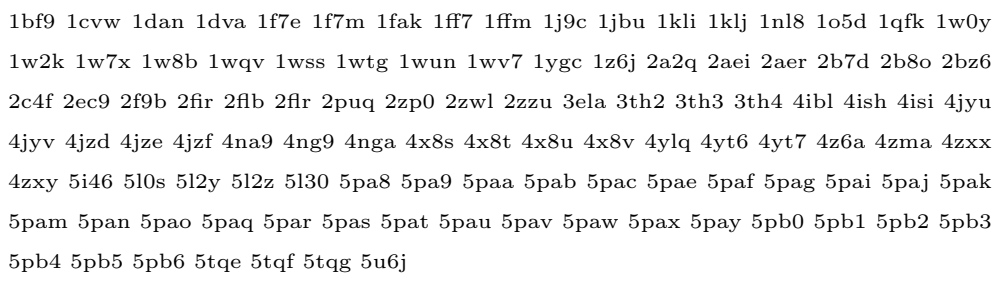 \\
\hline $\begin{array}{l}\text { Ferrochelatase/ Ferrochelatase/ } \\
\text { T42822 }\end{array}$ & $\begin{array}{l}\text { 1hrk } 2 \text { hrc } 2 \text { hre } 2 \text { pnj } 2 \text { po5 2po7 2qd1 2qd2 2qd3 2qd4 2qd5 3aqi 3hcn 3hco 3hcp 3hcr 3w1w } \\
4 \text { ffd } 4 \text { kla } 4 \text { klc } 4 \text { klr } 4 \mathrm{kmm} 4 \mathrm{mk} 4\end{array}$ \\
\hline $\begin{array}{l}\text { Gamma-aminobutyric acid B recep- } \\
\text { tor/ GPCR glutamate/ T } 42446\end{array}$ & 4f11 $4 \mathrm{f} 12 \quad 4 \mathrm{mqe} 4 \mathrm{mqf} 4 \mathrm{mr} 74 \mathrm{mr} 8 \quad 4 \mathrm{mr} 9 \quad 4 \mathrm{mrm}$ 4ms $14 \mathrm{~ms} 34 \mathrm{~ms} 4$ 4pas \\
\hline $\begin{array}{l}\text { 5-hydroxytryptamine } 2 \mathrm{~B} \text { receptor/ } \\
\text { GPCR rhodopsin/ T31204 }\end{array}$ & 4ib4 4nc3 5tud 5tvn \\
\hline $\begin{array}{l}\text { Protein kinase } \mathrm{C} \text {, theta type/ Ki- } \\
\text { nase/ T23995 }\end{array}$ & 1xjd 2 enj 2 enn 2 enz 2 jed $4 \mathrm{q} 9 \mathrm{z}$ 4ra5 5 f9e \\
\hline $\begin{array}{l}\text { Mitogen-activated protein kinase } 3 / \\
\text { Kinase/ T23276 }\end{array}$ & 2 zoq 4 qtb \\
\hline $\begin{array}{l}\text { 3-phosphoinositide dependent pro- } \\
\text { tein kinase-1/ Kinase/ T20891 }\end{array}$ & $\begin{array}{l}\text { 1h1w 1oky 1okz 1uu3 1uu7 1uu8 1uu9 1uvr 1w1d 1w1g 1w1h 1z5m 2biy 2pe0 2pe1 2pe2 } \\
\text { 2r7b 2vki 2xch 2xck 3h9o 3hrc 3hrf 3ion 3iop 3nax 3nay 3nun 3nus 3nuu 3nuy 3orx 3orz } \\
\text { 3otu 3pwy 3qc4 3qcq 3qcs 3qcx 3qcy 3qd0 3qd3 3qd4 3rcj 3rwp 3rwq 3sc1 4a06 4a07 4aw0 } \\
\text { 4aw1 4ct1 4ct2 4rqk 4rqv 4rrv 4xx9 5ack 5hkm 5hng 5ho7 5ho8 5lvl 5lvm 5lvn 5lvo 5lvp } \\
\text { 5mrd }\end{array}$ \\
\hline
\end{tabular}




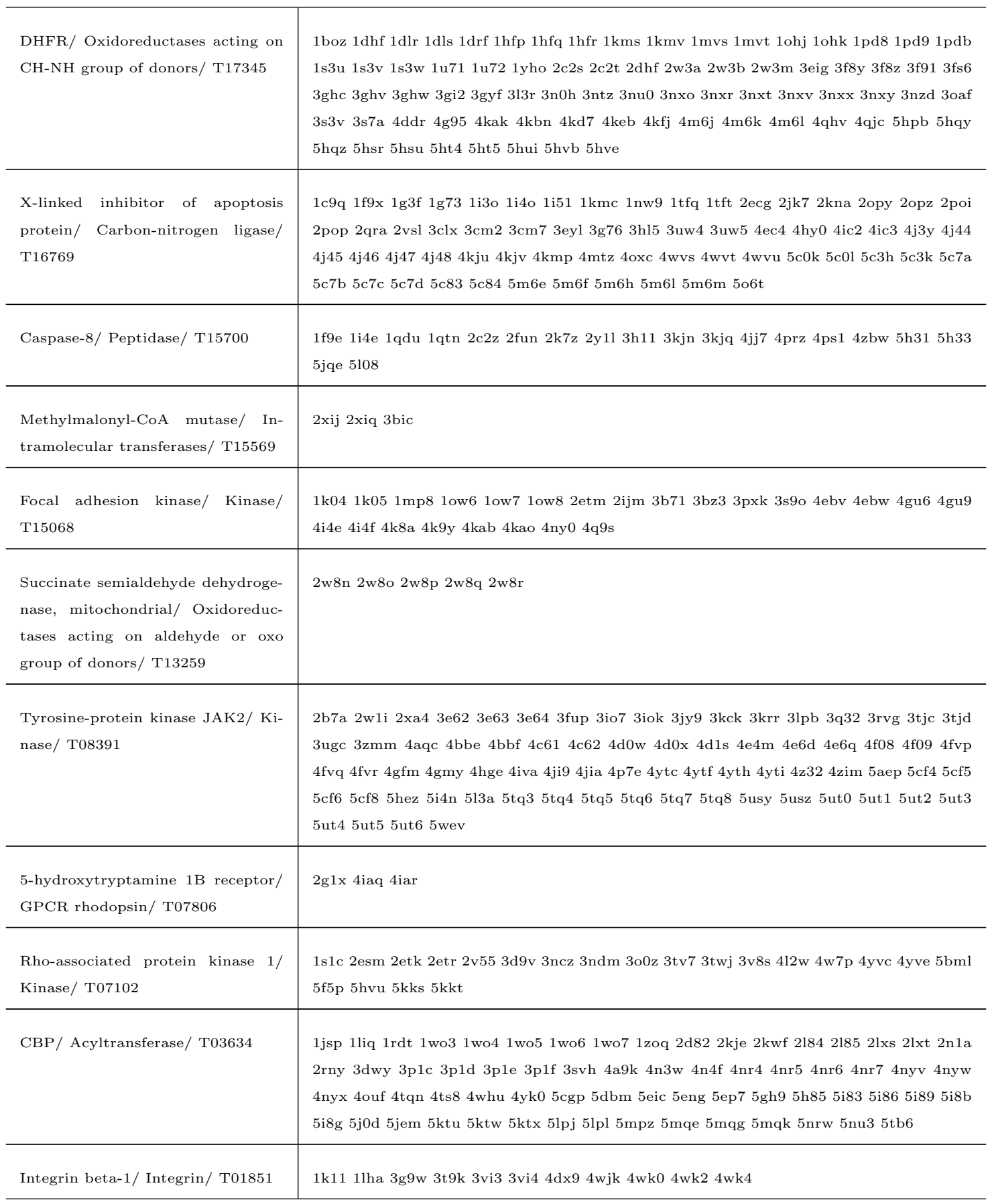




\begin{tabular}{|c|c|}
\hline $\begin{array}{l}\text { Dihydroorotate dehydrogenase, mi- } \\
\text { tochondrial/ Oxidoreductases act- } \\
\text { ing on CH-CH group of donors/ } \\
\text { T01318 }\end{array}$ & 1tv5 $3 \mathrm{i} 653 \mathrm{i} 68$ 3i6r 3o8a 3sfk 4cq8 4cq9 4cqa 4orm 4rx0 5boo 5del 5 fi 8 5tbo \\
\hline $\begin{array}{l}\text { Mitogen-activated protein kinase } \\
\text { 10/ Kinase/ T00663 }\end{array}$ & $\begin{array}{l}\text { 1jnk 1pmn 1pmu 1pmv 2b1p 2exc 2o0u 2o2u 2ok1 2p33 2r9s 2waj 2zdt 2zdu 3cgf 3cgo } \\
\text { 3da6 3fi2 3fi3 3fv8 3g90 3g9l 3g9n 3kvx 3oxi 3oy1 3ptg 3rtp 3tti 3ttj 3v6r 3v6s 4h36 4h39 } \\
\text { 4h3b 4kke 4kkg 4kkh 4u79 4w4v 4w4w 4w4x 4w4y 4whz 4x21 4y46 4y5h 4z9l }\end{array}$ \\
\hline $\begin{array}{l}\text { Ubiquitin-protein ligase E3 Mdm2/ } \\
\text { Carbon-nitrogen ligase/ T00176 }\end{array}$ & $\begin{array}{l}\text { 1rv1 1t4e 1t4f 1ycr 1z1m 2axi 2c6a 2c6b 2f1y 2fop 2gv2 2hdp 2lzg 2m86 2mps 2ruh 2vje } \\
\text { 2vjf 3eqs 3g03 3iux 3iwy 3jzk 3jzr 3jzs 3lbk 3lbl 3lnj 3lnz 3mqs 3tj2 3tpx 3tu1 3v3b 3vbg } \\
\text { 3vzv 3w69 4dij 4ere 4erf 4hbm 4hfz 4hg7 4jv7 4jv9 4jve 4jvr 4jwr 4mdn 4mdq 4oas 4oba } \\
\text { 4occ 4ode 4odf 4ogn 4ogt 4ogv 4oq3 4qo4 4qoc 4ud7 4ue1 4umn 4wt2 4xxb 4zfi 4zgk 4zyc } \\
\text { 4zyf 4zyi 5afg 5c5a 5hmh 5hmi 5hmk 5j7f 5j7g 5lav 5law 5lay 5laz } 5 \ln 25 \mathrm{mnj} 5 \text { swk } 5 \text { trf }\end{array}$ \\
\hline $\begin{array}{l}\text { Thyroid hormone receptor beta-1/ } \\
\text { Nuclear hormone receptor/ T } 98933\end{array}$ & $\begin{array}{l}\text { 1bsx 1n46 1nax 1nq0 1nq1 1nq2 1nuo 1q4x 1r6g 1xzx 1y0x 2j4a 2nll 2pin 3d57 3gws 3imy } \\
3 \text { jzc 4zo1 }\end{array}$ \\
\hline $\begin{array}{l}\text { Mitogen-activated protein kinase } \\
\text { kinase kinase 5/ Kinase/ T97589 }\end{array}$ & 2clq 3vw6 4bf2 4bhn 4bib 4bic 4bid 4bie 5ulm 5uor 5uox 5up3 5v19 5v24 6ej1 \\
\hline $\begin{array}{l}\text { UDP-glucose 4-epimerase/ Race- } \\
\text { mases and epimerases/ T96014 }\end{array}$ & 1ek5 1ek6 1hzj 1i3k 1i3l 1i3m 1i3n \\
\hline $\begin{array}{l}\text { Retinoic acid receptor alpha/ } \mathrm{Nu}- \\
\text { clear hormone receptor/ T94085 }\end{array}$ & $1 \mathrm{dkf} 1 \mathrm{dsz} 3 \mathrm{a} 9 \mathrm{e} 3 \mathrm{kmr} 3 \mathrm{kmz} 4 \mathrm{dqm} 5 \mathrm{k} 13$ \\
\hline FGF-3 receptor/ Kinase/ T91331 & 1ry7 2lzl $4 \mathrm{k} 33$ \\
\hline $\begin{array}{l}\text { DNMT1/ Methyltransferase super- } \\
\text { family/ T } 88304\end{array}$ & 3epz 3pta 3swr 4wxx 4yoc 4z96 4z97 \\
\hline Aurora kinase A/ Kinase/ T 87675 & 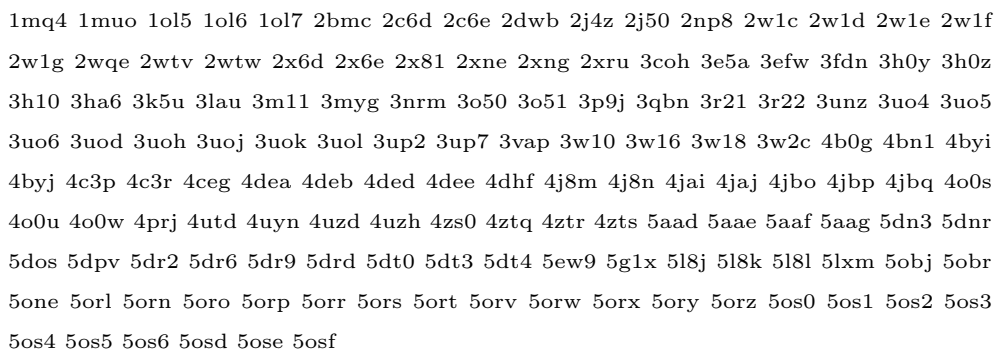 \\
\hline $\begin{array}{l}\text { Receptor protein-tyrosine kinase } \\
\text { erbB-3/ Kinase/ T } 86350\end{array}$ & 1m6b 219u 3kex 3lmg 3p11 4leo 4otw 4p59 4riw 4rix 4riy 5cus \\
\hline
\end{tabular}




\begin{tabular}{|c|c|}
\hline Thioredoxin/ Thioredoxin/ T85616 & $\begin{array}{l}\text { 1aiu 1auc 1cqg 1cqh 1ert 1eru 1erv 1erw 1m7t 1mdi 1mdj 1mdk 1trs } 1 \text { tru 1trv 1trw 1w1c } \\
\text { 1w1e 2hsh 2hxk 2ifq 2iiy 3e3e 3kd0 3m9j 3m9k 3qfa 3qfb 3trx } 411141144 \text { oo4 4oo5 4pok } \\
\text { 4pol 4pom 4puf 4trx 5dqy }\end{array}$ \\
\hline $\begin{array}{l}\text { Glutamate receptor, ionotropic } \\
\text { kainate } 1 / \text { Glutamate-gated ion } \\
\text { channel/ T73495 }\end{array}$ & 2 zns 2 znt $2 \mathrm{znu} 3 \mathrm{fuz} 3 \mathrm{fv} 13 \mathrm{fv} 23 \mathrm{fvg} 3 \mathrm{fvk} 3 \mathrm{fvn} 3 \mathrm{fvo} 4 \mathrm{mf} 3$ \\
\hline $\begin{array}{l}\text { Steroid hormone receptor ERR1/ } \\
\text { Nuclear hormone receptor/ T72841 }\end{array}$ & $1 \mathrm{xb} 72 \mathrm{pj} 13 \mathrm{~d} 243 \mathrm{k} 6 \mathrm{p}$ \\
\hline $\begin{array}{l}\text { mRNA of Protein kinase C-theta/ } \\
\text { Kinase/ T72295 }\end{array}$ & 1 xjd 2 enj 2 enn 2 enz 2 jed $4 q 9 \mathrm{z} 4 \mathrm{ra} 5$ ffee \\
\hline $\begin{array}{l}\text { Wee1-like protein kinase/ Kinase/ } \\
\text { T69991 }\end{array}$ & 1x8b 2in6 2io6 2z2w 3bi6 3biz 3cqe 3cr0 5v5y 5vc3 5vc4 5vc5 5vc6 5vd2 \\
\hline Cathepsin S/ Peptidase/ T68290 & $\begin{array}{l}\text { 1bxf 1glo } 1 \mathrm{~ms} 6 \text { 1npz 1nqc 2c0y 2f1g 2fq9 } 2 \text { fra } 2 \text { frq } 2 \mathrm{ft} 2 \text { 2fud } 2 \text { fye } 2 \mathrm{~g} 6 \mathrm{~d} 2 \mathrm{~g} 7 \mathrm{y} 2 \mathrm{~h} 7 \mathrm{j} 2 \mathrm{hh} 5 \\
2 \mathrm{hhn} 2 \mathrm{hxz} 2 \mathrm{op} 32 \mathrm{r} 9 \mathrm{~m} \text { 2r9n 2r9o 3iej 3kwn 3mpe 3mpf 3n3g 3n4c 3ovx } 4 \mathrm{p} 6 \mathrm{e} 4 \mathrm{p} 6 \mathrm{~g}\end{array}$ \\
\hline $\begin{array}{l}\text { 5'-Nucleotidase/ Phosphoric mo- } \\
\text { noester hydrolases / T65116 }\end{array}$ & $4 \mathrm{~h} 1 \mathrm{~s} 4 \mathrm{~h} 1 \mathrm{y} 4 \mathrm{~h} 2 \mathrm{~b} 4 \mathrm{~h} 2 \mathrm{f} 4 \mathrm{~h} 2 \mathrm{~g} 4 \mathrm{~h} 2 \mathrm{i}$ \\
\hline $\begin{array}{l}\text { Serine/ threonine-protein kinase } \\
\text { Sgk1/ T64682 }\end{array}$ & $2 \mathrm{r} 5 \mathrm{t} 3 \mathrm{hdm} 3 \mathrm{hdn}$ \\
\hline $\begin{array}{l}\text { Serine/ threonine-protein kinase } \\
\text { Chk1/ T62449 }\end{array}$ & 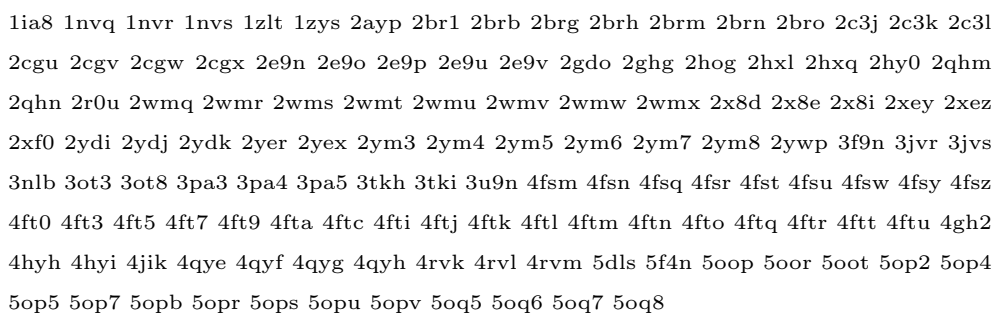 \\
\hline $\begin{array}{l}\text { Dihydroorotate dehydrogenase/ } \\
\text { Oxidoreductases acting on } \mathrm{CH}-\mathrm{CH} \\
\text { group of donors/ T61400 }\end{array}$ & 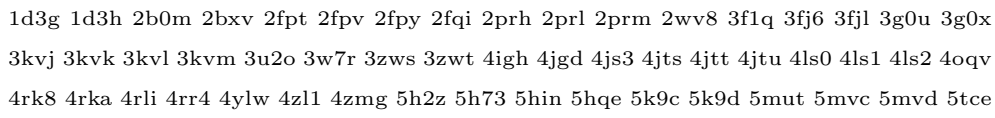 \\
\hline $\begin{array}{l}\text { Proto-oncogene tyrosine-protein ki- } \\
\text { nase receptor ret/ Kinase/ T60631 }\end{array}$ & 1xpd 2 ivs 2 ivt 2 ivu 2 ivv $2 \times 2 \mathrm{k} 2 \times 212 \times 2 \mathrm{~m} 2 \times 2 \mathrm{u} 4$ cki 4 ckj 4 ux 85 amn $5 \mathrm{fm} 25 \mathrm{fm} 3$ \\
\hline $\begin{array}{l}\text { Cell division protein kinase } 7 / \mathrm{Ki}- \\
\text { nase/ T58449 }\end{array}$ & $1 \lg 3$ pa 8 ua2 2hic \\
\hline
\end{tabular}




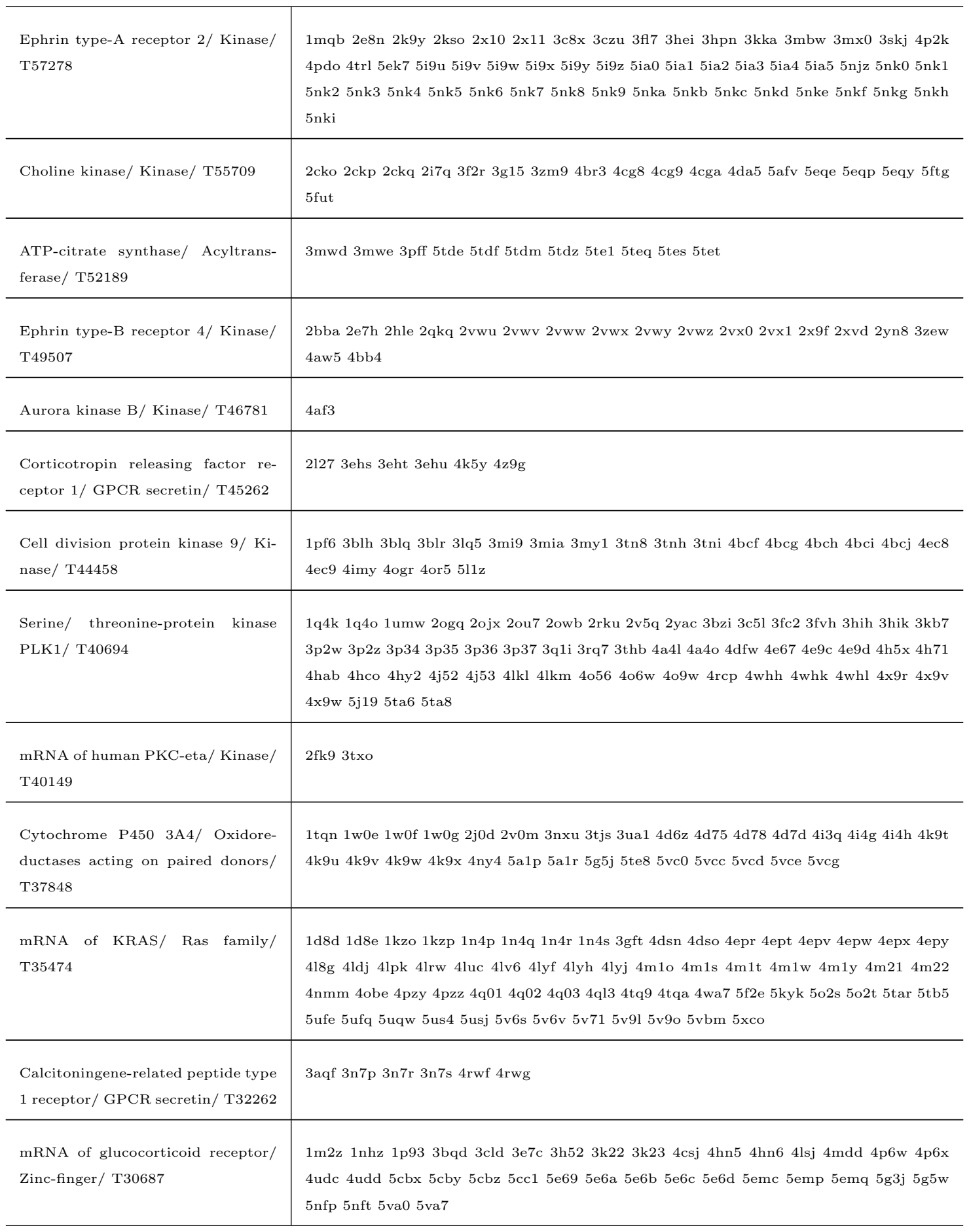




\begin{tabular}{|c|c|}
\hline $\begin{array}{l}\text { mRNA of human caspase 6/ Pepti- } \\
\text { dase/ T25927 }\end{array}$ & $\begin{array}{l}\text { 1mi9 2wdp 3k7e 3nkf 3nr2 3od5 3p45 3p4u 3qnw 3s70 3s 8e 3v6l 3v6m 4ejf 4fxo 4hva 4iyr } \\
4 \mathrm{n} 5 \mathrm{~d} 4 \mathrm{n} 6 \mathrm{~g} 4 \mathrm{n} 7 \mathrm{j} 4 \mathrm{n} 7 \mathrm{~m} 4 \mathrm{nbk} 4 \mathrm{nbl} 4 \mathrm{nbn}\end{array}$ \\
\hline PFKFB3 kinase/ Kinase/ T23787 & $\begin{array}{l}\text { 2axn } 2 \text { dwo } 2 \text { dwp } 2 \mathrm{i} 1 \mathrm{v} 3 \mathrm{qpu} 3 \mathrm{qpv} 3 \mathrm{qpw} 4 \mathrm{~d} 4 \mathrm{j} 4 \mathrm{~d} 4 \mathrm{k} 4 \mathrm{~d} 4 \mathrm{l} 4 \mathrm{~d} 4 \mathrm{~m} 4 \mathrm{ma} 45 \mathrm{ajv} 5 \mathrm{ajw} 5 \mathrm{ajx} 5 \mathrm{ajy} \\
5 \text { ajz } 5 \text { ak0 }\end{array}$ \\
\hline $\begin{array}{l}\text { Cytochrome P450 11A1, mitochon- } \\
\text { drial/ Oxidoreductases acting on } \\
\text { paired donors/ T23145 }\end{array}$ & $3 \mathrm{n} 9 \mathrm{y} 3 \mathrm{n} 9 \mathrm{z}$ 3na0 3na1 \\
\hline $\begin{array}{l}\text { MAPKAP kinase } 2 / \text { Kinase/ } \\
\text { T21307 }\end{array}$ & $\begin{array}{l}\text { 1kwp } 1 \mathrm{nxk} 1 \mathrm{ny} 32 \mathrm{jbo} 2 \mathrm{jbp} 2 \mathrm{okr} 2 \mathrm{onl} 2 \mathrm{oza} 2 \mathrm{p} 3 \mathrm{~g} 2 \mathrm{pzy} 3 \mathrm{a} 2 \mathrm{c} 3 \mathrm{fpm} 3 \mathrm{fyj} 3 \mathrm{fyk} 3 \mathrm{gok} 3 \mathrm{ka0} 3 \mathrm{kc} 3 \\
3 \mathrm{kga} 3 \mathrm{~m} 2 \mathrm{w} 3 \mathrm{~m} 42 \text { 3r2b 3r2y 3r30 3wi6 4tyh }\end{array}$ \\
\hline $\begin{array}{l}\text { Cell division protein kinase } 5 / \mathrm{Ki}- \\
\text { nase/ T20973 }\end{array}$ & 1h4l 1lfr 1ung 1 unh 1 unl $3 o 0 g$ au 8 \\
\hline $\begin{array}{l}\text { mRNA of PKA Catalytic Subunit } \\
\text { C-alpha/ Kinase/ T20669 }\end{array}$ & $\begin{array}{l}\text { 2gu8 3agl 3agm 3ama 3amb } 3191 \text { 319m 319n 3mvj 3nx8 3oog 3ovv 3owp 3oxt 3p0m 3poo } \\
\text { 3vqh 4ae6 4ae9 4uj1 4uj2 4uj9 4uja 4ujb 4wb5 4wb6 4wb7 4wb8 5bx6 5bx7 5izf 5izj 5j5x }\end{array}$ \\
\hline
\end{tabular}


Carbonic anhydrase II/ Carbonoxygen lyases/ T20401 12ca 1a42 1am6 1avn 1bcd 1bic 1bn1 1bn3 1bn4 1bnm 1bnn 1bnq 1bnt 1bnu 1bnv 1bnw $1 \mathrm{bv} 3$ 1ca2 1ca3 1cah 1cai 1caj 1cak 1cal 1cam 1can 1cao 1cay 1caz 1ccs 1cct 1ccu 1cil $1 \mathrm{cim} 1 \mathrm{cin} 1 \mathrm{cnb} 1 \mathrm{cnc} 1 \mathrm{cng} 1 \mathrm{cnh} 1 \mathrm{cni} 1 \mathrm{cnj} 1 \mathrm{cnk} 1 \mathrm{cnw} 1 \mathrm{cnx} 1 \mathrm{cny} 1 \mathrm{cra} 1 \mathrm{cva} 1 \mathrm{cvb} 1 \mathrm{cvc} 1 \mathrm{cvd}$ $1 \mathrm{cve} 1 \mathrm{cvf} 1 \mathrm{cvh} 1 \mathrm{dca} 1 \mathrm{dcb} 1 \mathrm{eou} 1 \mathrm{f} 2 \mathrm{w} 1 \mathrm{fql} 1 \mathrm{fqm} 1 \mathrm{fqn} 1 \mathrm{fqr} 1 \mathrm{fr} 41 \mathrm{fr} 7 \mathrm{fsn} 1 \mathrm{fsq} 1 \mathrm{fsr} 1 \mathrm{~g} 0 \mathrm{e} 1 \mathrm{~g} 0 \mathrm{f}$ 1g1d 1g3z 1g45 1g46 1g48 1g4j 1g4o 1g52 1g53 1g54 1h4n 1h9n 1h9q 1hca 1hea 1heb 1hec 1hed 1hva 1i8z 1 i90 1i91 1i9l 1i9m 1i9n 1i9o 1i9p 1i9q 1if4 1if5 1if6 1if7 1if8 1if9 1kwq $1 \mathrm{kwr} 1 \lg 5$ 1lg6 1lgd 1lug 1lzv 1moo 1mua 1okl 1okm 1okn 1oq5 1ray 1raz 1rza 1rzb 1rzc 1rzd 1rze 1t9n 1tb0 1tbt 1te3 1 teq 1 teu 1 tg 3 1tg9 1 th9 1 thk 1 ttm 1 uga 1 ugb 1 ugc 1 ugd 1uge 1ugf 1ugg 1xeg 1xev 1xpz 1xq0 1yda 1ydb 1ydc 1ydd 1yo0 1yo1 1yo2 1z9y 1ze8 1zfk 1zfq 1zge 1zgf 1zh9 1zsa 1zsb 1zsc 2abe 2aw1 2ax2 2ca2 2cba 2cbb 2cbc 2cbd 2cbe 2eu2 2eu3 2ez7 2 f14 2 fmg 2 fmz 2 fnk 2 fnm 2 fnn 2 foq 2 fos 2 fou 2 fov 2 gd8 2 geh 2 h 15 2h4n 2 hd6 2hkk 2hl4 2hnc 2hoc 2ili 2nng 2nno 2nns 2nnv 2nwo 2nwp 2nwy 2nwz 2nxr 2nxs 2nxt 2o4z 2osf 2osm 2pou 2pov 2pow 2q1b 2q1q 2q38 2qo8 2qoa 2qp6 2vva 2vvb 2wd2 2wd3 2weg 2weh 2wej 2weo 2x7s 2x7t 2x7u 3b4f 3bet 3bl0 3bl1 3c7p 3ca2 3caj 3cyu 3d8w 3d92 $3 \mathrm{~d} 93$ 3d9z 3daz 3dbu 3dc3 3dc9 3dcc 3dcs 3dcw 3dd0 3dd8 3dv7 3dvb 3dvc 3dvd 3efi 3eft 3f4x 3f8e 3ffp 3gz0 3hfp 3hkn 3hkq 3hkt 3hku 3hlj 3hs4 3ibi 3ibl 3ibn 3ibu 3ieo 3igp 3k2f $3 \mathrm{k} 34$ 3k7k 3kig 3kkx 3kne 3koi 3kok 3kon 3ks3 3kwa $31143 \mathrm{~m} 04$ 3m14 3m1j 3m1k 3m1q 3m1w 3m2n 3m2x 3m2y 3m2z 3m3x 3m40 3m5e 3m5s 3m5t 3m67 3m96 3m98 3mhc 3mhi $3 \mathrm{mhl} 3 \mathrm{mhm} 3 \mathrm{mho} 3 \mathrm{ml} 23 \mathrm{mmf} 3 \mathrm{mna} 3 \mathrm{mnh} 3 \mathrm{mni} 3 \mathrm{mnj} 3 \mathrm{mnk} 3 \mathrm{mnu} 3 \mathrm{mwo} 3 \mathrm{myq} 3 \mathrm{mzc} 3 \mathrm{non}$ 3n2p 3n3j 3n4b 3nb5 3ni5 3nj9 3oik 3oil 3oim 3oku 3okv 3oy0 3oyq 3oys 3p3h 3p3j 3p44 3p4v 3p55 3p58 3p5a 3p5l 3pjj 3po6 3pyk 3qyk 3r16 3r17 3rg3 3rg4 3rge 3rj7 3rld 3ryj 3ryv 3ryx 3ryy 3ryz 3rz0 3rz1 3rz5 3rz7 3rz8 3s71 3s72 3s73 3s74 3s75 3s76 3s77 3s78 3s8x 3s9t 3sap 3sax 3sbh 3sbi 3t5u 3t5z 3t82 3t83 3t84 3t85 3tmj 3tvn 3tvo 3u3a 3u45 3u47 3u7c 3v2j 3v2m 3v3f 3v3g 3v3h 3v3i 3v3j 3v5g 3v7x 3vbd 3zp9 4bcw 4bf1 4bf6 4ca2 4cac 4cq0 4dz7 4dz9 4e3d 4e3f 4e3g 4e3h 4e49 4e4a 4e5q 4fik 4fl7 4fpt 4frc 4fu5 4fvn 4fvo 4g0c $4 \mathrm{gl1} 4 \mathrm{hba} 4 \mathrm{hew} 4 \mathrm{hey} 4 \mathrm{hez} 4 \mathrm{hf} 34 \mathrm{ht0} 4 \mathrm{idr} 4 \mathrm{ilx} 4$ ito 4 itp $4 \mathrm{iwz} 4 \mathrm{js} 6$ 4jsa 4 jss 4 jsw $4 \mathrm{jsz} 4 \mathrm{k} 0 \mathrm{~s}$ 4k0t 4k0z 4k13 4k1q 4kap 4kni 4knj 4kuv 4kuw 4kuy 4kv0 415u 415v 415w 4lhi 4lp6 4m2r $4 \mathrm{~m} 2 \mathrm{u} 4 \mathrm{~m} 2 \mathrm{v} 4 \mathrm{~m} 2 \mathrm{w} 4 \mathrm{mdg} 4 \mathrm{mdl} 4 \mathrm{mdm} 4 \mathrm{mlt} 4 \mathrm{mlx}$ 4mo8 4mty 4n0x 4n16 4pq7 4pxx 4pyx 4pyy 4pzh 4q06 4q07 4q08 4q09 4q49 4q6d 4q6e 4q78 4q7p 4q7s 4q7v 4q7w 4q81 4q83 4q87 4q8x 4q8y 4q8z 4q90 4q99 4q9y 4qef 4qiy 4qjm 4qk1 4qk2 4qk3 4qsa 4qsb 4qsi 4qt 4qy3 4r59 4r5a 4r5b 4rfc 4rfd 4rh2 4riu 4riv 4rn4 4rux 4ruy 4ruz 4wl4 4ww6 4xe1 4y0j 4ygj 4ygk 4ygl 4ygn 4yvy 4ywp 4yx4 4yxi 4yxo 4yxu 4yyt 4z0q 4z1e 4z1j 4z1k 4z1n 4zao 4zwi 4zwx 4zwy 4zwz 4zx0 4zx1 5a6h 5amd 5amg 5aml 5bnl 5bru 5brv 5brw 5byi 5c8i $5 \mathrm{ca} 25 \mathrm{cac} 5 \mathrm{cj} 15 \mathrm{clu} 5 \mathrm{dog} 5 \mathrm{doh} 5 \mathrm{drs} 5 \mathrm{dsi} 5 \mathrm{dsj} 5 \mathrm{dsk} 5 \mathrm{dsl} 5 \mathrm{dsm} 5 \mathrm{dsn} 5$ dso 5 dsp 5 dsq 5 dsr $5 \mathrm{e} 28$ 5e2k 5e2r 5e2s 5eh5 5eh7 5eh8 5ehe 5ehv 5ehw 5eij 5ekh 5ekj 5ekm 5eoi 5fdc 5fdi 5 flo $5 \mathrm{flp} 5 \mathrm{flq} 5 \mathrm{flr} 5 \mathrm{fls} 5 \mathrm{flt} 5 \mathrm{fng} 5 \mathrm{fnh} 5 \mathrm{fni} 5 \mathrm{fnj} 5 \mathrm{fnk} 5 \mathrm{fnl} 5 \mathrm{fnm} 5 \mathrm{~g} 015 \mathrm{~g} 035 \mathrm{~g} 0 \mathrm{~b} 5 \mathrm{~g} 0 \mathrm{c} 5 \mathrm{gmn}$ 5 j8z 5 jdv 5 je 75 jeg 5 jeh 5 jep 5 jes 5 jg 3 5jg 5 5jgs 5 jgt 5 jmz 5 jn 15 jn 35 jn 7 jjq0 5 jqt 5130 5l6k 5l6t 5170 519e 5ljq 5ljt 51145118 5llc 5lle 5llg 5llh 5lmd 5lvs 5n0d 5n0e 5o07 5sz0 5sz1 $5 \mathrm{sz} 25 \mathrm{sz} 35 \mathrm{sz} 45 \mathrm{sz} 5$ 5sz6 5sz7 5t71 5t72 5t74 5t75 5tfx 5th4 5thi 5thj 5thn 5 ti0 5 txy 5 ty 1 5 ty 8 ty 9 tya 5u0d 5u0e 5u0f 5u0g 5uln 5vgy 5wex 6ca2 7ca2 8ca2 9ca2

\begin{tabular}{|c|c|}
\hline $\begin{array}{l}\text { Glutathione } \\
\text { tramolecular } \\
\text { T19433 }\end{array}$ & $\begin{array}{l}\text { 1iyh 1iyi } 1 \mathrm{v} 402 \mathrm{cvd} 2 \mathrm{vcq} 2 \mathrm{vcw} 2 \mathrm{vcx} 2 \mathrm{vcz} 2 \mathrm{vd} 02 \mathrm{vd} 13 \mathrm{ee} 23 \mathrm{kxo} 3 \mathrm{vi} 53 \mathrm{vi} 74 \mathrm{ec} 04 \mathrm{edy} 4 \mathrm{edz} \\
4 \mathrm{ee} 0 \text { 5ais } 5 \text { aiv } 5 \text { aix }\end{array}$ \\
\hline $\begin{array}{l}\text { Phospholipase A2, membrane asso- } \\
\text { ciated/ Carboxylic ester hydrolase/ } \\
\text { T19160 }\end{array}$ & $\begin{array}{l}\text { 1ayp } 1 \mathrm{bbc} 1 \mathrm{db} 41 \mathrm{db} 51 \mathrm{dcy} 1 \mathrm{j} 1 \mathrm{a} 1 \mathrm{kqu} 1 \mathrm{kvo} 1 \mathrm{n} 28 \text { 1n29 1pod 1poe } 2 \text { gny } 3 \mathrm{u} 8 \mathrm{~b} 3 \mathrm{u} 8 \mathrm{~d} 3 \mathrm{u} 8 \mathrm{~h} \\
3 \mathrm{u} 8 \mathrm{i} 5 \mathrm{~g} 3 \mathrm{n}\end{array}$ \\
\hline
\end{tabular}




\begin{tabular}{|c|c|}
\hline $\begin{array}{l}\text { Histamine N-methyltransferase/ } \\
\text { Methyltransferase superfamily/ } \\
\text { T17448 }\end{array}$ & 1icz 1jqd 1jqe 2aot 2aou 2aov 2aow 2aox \\
\hline $\begin{array}{l}\text { Protein tyrosine phosphatase-1B/ } \\
\text { Phosphoric monoester hydrolases/ } \\
\text { T16347 }\end{array}$ & 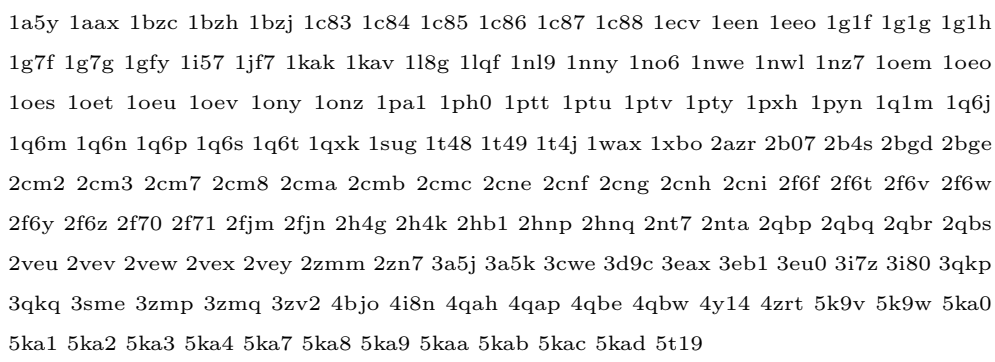 \\
\hline $\begin{array}{l}\text { Integrin-linked protein kinase } 1 / \\
\text { Kinase/ T13616 }\end{array}$ & $2 \mathrm{kbx} 3 \mathrm{f} 6 \mathrm{q}$ 3ixe $3 \mathrm{kmu} 3 \mathrm{kmw}$ 3rep 4hi8 4hi9 \\
\hline $\begin{array}{l}\text { CAMP-specific } 3^{\prime}, 5^{\prime} \text {-cyclic phos- } \\
\text { phodiesterase } 4 \mathrm{~B} / \text { Phosphoric di- } \\
\text { ester hydrolases/ T10265 }\end{array}$ & 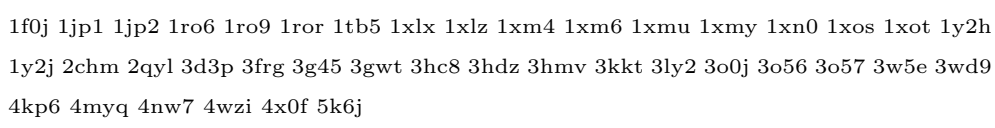 \\
\hline $\begin{array}{l}\text { Neutrophil collagenase/ Peptidase/ } \\
\text { T08856 }\end{array}$ & 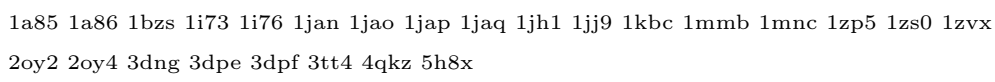 \\
\hline Granzyme B/ Peptidase/ T08298 & $1 \mathrm{fq} 3 \mathrm{iau}$ \\
\hline $\begin{array}{l}\text { Focal adhesion kinase-2/ Kinase/ } \\
\text { T07087 }\end{array}$ & $\begin{array}{l}\text { 2fo6 } 2 \mathrm{lk} 4 \text { 3cc6 3et7 3fzo 3fzp 3fzr 3fzs 3fzt 3gm1 3gm2 3gm3 3h3c 3u3f 4eku 4h1j 4h1m } \\
4 \mathrm{r} 32 \text { 4xef 4xek 4xev 5to8 }\end{array}$ \\
\hline $\begin{array}{l}\text { Neutral endopeptidase/ Peptidase/ } \\
\text { T05409 }\end{array}$ & 1dl9 1dmt 1qvd 1r1h 1r1i 1r1j 1y8j 2qpj 2yb9 4cth 5jmy \\
\hline $\begin{array}{l}\text { Mitogen-activated protein kinase } \\
\text { kinase kinase } 7 / \text { Kinase/ T04361 }\end{array}$ & $\begin{array}{l}\text { 2eva 2yiy 4gs6 4l3p } 415241534 \text { o91 5e7r 5gjd 5gjf 5gjg 5j7s 5j8i 5j9l 5jga 5jgb 5jgd 5jh6 } \\
5 \text { jk3 5v5n }\end{array}$ \\
\hline $\begin{array}{l}\text { mRNA of H-ras/ Ras family/ } \\
\text { T03871 }\end{array}$ & 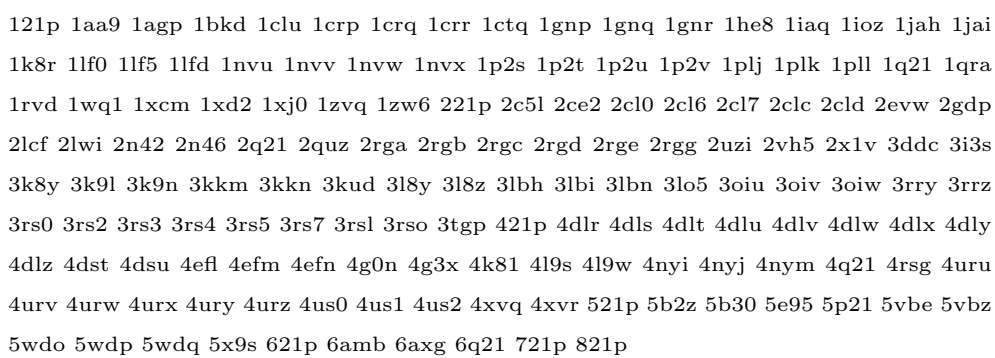 \\
\hline
\end{tabular}


mRNA of PPAR-gamma/ Nuclear hormone receptor/ T03818
$1 \mathrm{fm} 61 \mathrm{fm} 9$ 1i7i $1 \mathrm{k} 74$ 1 knu 1nyx 1prg 1rdt 1wm0 1zeo 1zgy 2ath 2f4b 2fvj 2g0g 2g0h 2gtk 2hfp 2hwq 2hwr 2i4j 2i4p 2i4z 2om9 2p4y 2pob 2prg 2q59 2q5p 2q5s 2q61 2q6r 2q6s 2q8s 2qmv 2vsr 2vst 2vv0 2vv1 2vv2 2vv3 2vv4 2xkw 2yfe 2zk0 2zk1 2zk2 2zk3 2zk4 2zk5 2zk6 2zno 2zvt 3ads 3adt 3adu 3adv 3adw 3adx 3an3 3an4 3b0q 3b0r 3b1m 3b3k 3bc5 3cdp 3cds 3cs8 3cwd 3d6d 3dzu 3dzy 3e00 3et0 3et3 3fej 3fur 3g9e 3gbk 3h0a 3ho0 3hod 3ia6 3k8s 3kmg 3lmp 3noa 3osi 3osw 3pba 3po9 3prg 3qt0 3r5n 3r8a 3r8i 3s9s 3sz1 3t03 3ty0 3u9q 3v9t 3v9v 3v9y 3vjh 3vji 3vn2 3vso 3vsp 3wj4 3wj5 3wmh 3x1h 3x1i 4a4v 4a4w 4ci5 4e4k 4e4q 4em9 4ema 4f9m 4fgy 4hee 4jaz 4j14 41964198 4o8f 4oj4 4prg 4pvu 4pwl 4r06 4r2u 4r6s 4xld 4xta 4xuh 4xum 4y29 4yt1 5azv 5dsh 5dv3 5dv6 5dv8 5dvc 5dwl 5f9b 5gtn 5 gto 5 gtp 5 hzc 5 ji0 5lsg 5tto 5two 5 u 5 l

Type IV phosphodiesterase/ Phosphoric diester hydrolases/ T02001

1e9k 1mkd 1oyn 1ptw 1q9m 1tb7 1tbb 1xom 1xon 1xoq 1xor 1y2b 1y2c 1y2d 1y2e 1y2k 1zkn 2fm0 2fm5 2pw3 2qyn 3g4g 3g4i 3g4k 3g4l 3g58 3iad 3iak 3k4s 3sl3 3sl4 3sl5 3sl6 $3 \mathrm{~s} 18$ 3v9b 4 ogb 4 w1o 4 wcu $5 \mathrm{k} 1 \mathrm{i} 5 \mathrm{k} 32$ 5tkb 5 wqa

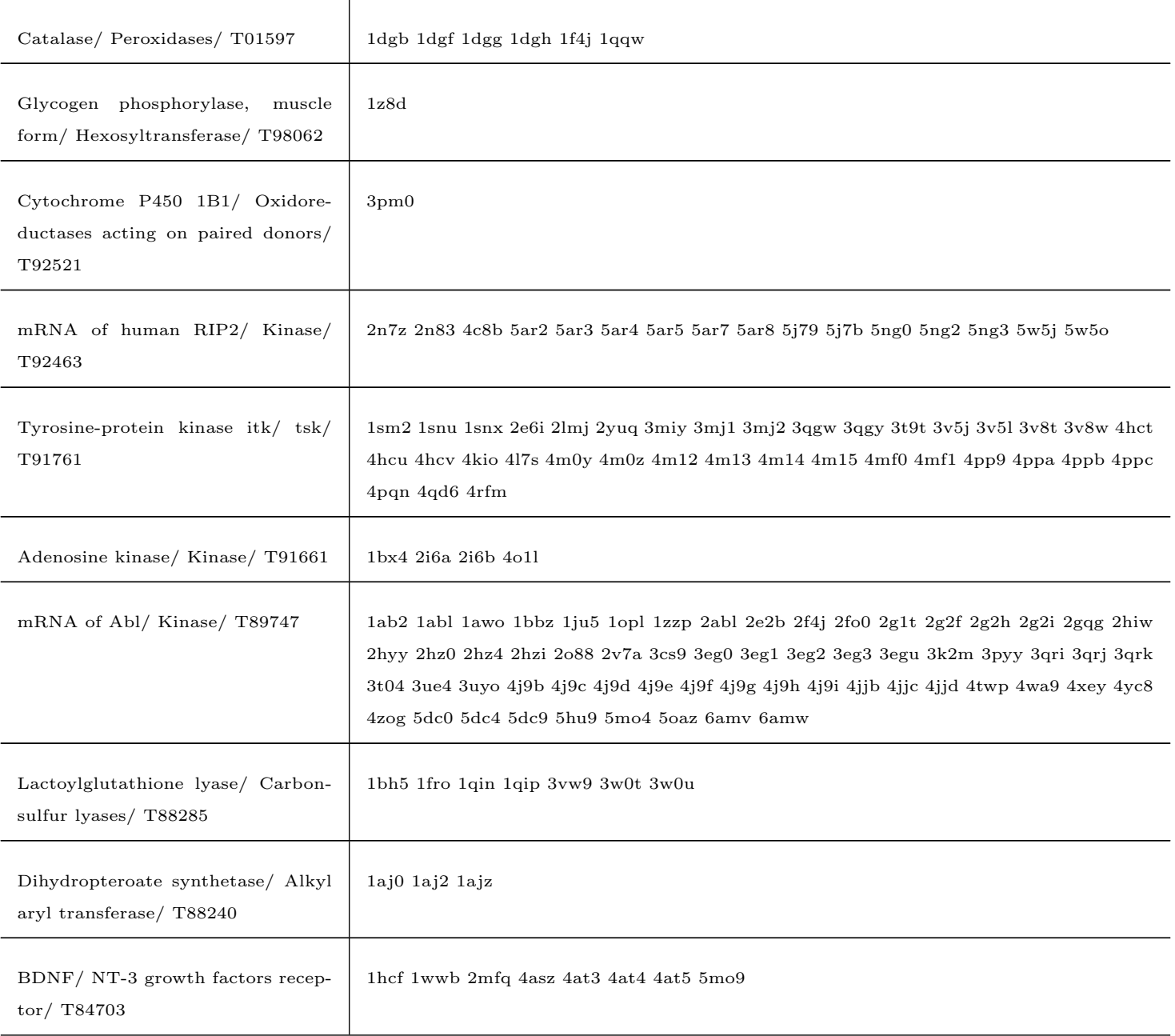




\begin{tabular}{|c|c|}
\hline $\begin{array}{l}\text { Glucosylceramidase/ Glycosylases/ } \\
\text { T84173 }\end{array}$ & $\begin{array}{l}\text { 1ogs } 1 \text { y7v } 2 \text { f61 2j25 2nsx 2nt0 2nt1 2v3d 2v3e 2v3f 2vt0 2wcg 2wkl 2xwd 2xwe 3gxd 3gxf } \\
3 \text { gxi 3gxm 3ke0 3keh 3rik 3ril 5lvx }\end{array}$ \\
\hline $\begin{array}{l}\text { Protein kinase C iota/ Kinase/ } \\
\text { T83174 }\end{array}$ & 1vd2 1wmh 1zrz 3a8w 3a8x 3zh8 5li1 5li9 5lih \\
\hline $\begin{array}{l}\text { Insulin-degrading Enzyme/ Pepti- } \\
\text { dase/ T81900 }\end{array}$ & 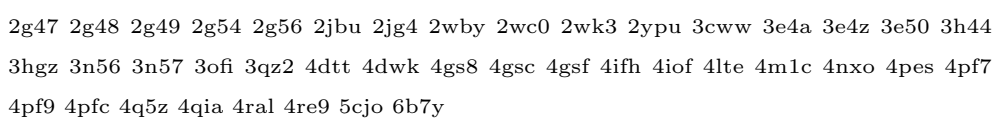 \\
\hline $\begin{array}{l}\text { mRNA of VEGFR1/ Kinase/ } \\
\text { T80782 }\end{array}$ & 1ftt 1qsv 1qsz 1qty 1rv6 2xac 3hng 4ckv 4cl7 5abd 5ex3 5t89 \\
\hline $\begin{array}{l}\text { Tryptophan 5-hydroxylase-1/ Ox- } \\
\text { idoreductases acting on paired } \\
\text { donors/ T78915 }\end{array}$ & 1 in $91 \mathrm{mlw} 3 \mathrm{hf} 63 \mathrm{hf} 83 \mathrm{hfb} 5 \mathrm{j} 6 \mathrm{~d} 51015 \mathrm{tpg}$ \\
\hline $\begin{array}{l}78 \mathrm{kDa} \text { glucose-regulated protein/ } \\
\text { Heat shock protein/ T77594 }\end{array}$ & 3iuc 3ldl 3ldn 3ldo 3ldp 5e84 5e 85 5e 86 5evz 5ex 5 exw 5ey 4 fox 5f1x 5f2r \\
\hline $\begin{array}{l}\text { CGMP-dependent3',5'-cyclic phos- } \\
\text { phodiesterase/ Phosphoric diester } \\
\text { hydrolases/ T77400 }\end{array}$ & 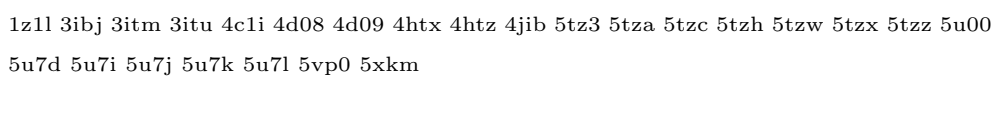 \\
\hline Pyridoxal kinase/ Kinase/ T76522 & 2ajp 2f7k 2yxt 2 yxu 3 fhx 3 fhy 3 keu 4 en 4 eoh \\
\hline $\begin{array}{l}\text { 6-phosphogluconate dehydrogenase, } \\
\text { decarboxylating/ Short-chain dehy- } \\
\text { drogenases reductases/ T76497 }\end{array}$ & $2 \mathrm{jkv} 4 \mathrm{gwg} 4 \mathrm{gwk}$ \\
\hline $\begin{array}{l}\text { Liver carboxylesterase/ Carboxylic } \\
\text { ester hydrolase/ T } 76369\end{array}$ & $\begin{array}{l}\text { 1mx1 1mx5 1mx9 1ya4 1ya8 1yah 1yaj 2dqy 2dqz 2dr0 2h7c 2hrq 2hrr 3k9b 4ab1 5a7f } \\
5 \mathrm{a} 7 \mathrm{~g} 5 \mathrm{a} 7 \mathrm{~h}\end{array}$ \\
\hline $\begin{array}{l}\text { GMP synthase } \quad \text { [glutamine- } \\
\text { hydrolyzing]/ Carbon-nitrogen } \\
\text { ligase/ T75517 }\end{array}$ & $2 \mathrm{vpi} 2 \mathrm{vxo}$ \\
\hline mRNA of CCR5/ ddRNAi/ T72171 & 1nd8 1ne0 1opn 1opt 1opw $21872 \mathrm{mzx} 2$ rll $2 \mathrm{rrs} 4 \mathrm{mbs}$ \\
\hline $\begin{array}{l}\text { Bifunctional aminoacyl tRNA } \\
\text { synthetase/ Carbon-oxygen ligase/ } \\
\text { T67416 }\end{array}$ & 1 fyj 4 hvc $4 \mathrm{k} 864 \mathrm{k} 874 \mathrm{k} 88$ aa1n $5 \mathrm{a} 345 \mathrm{a} 5 \mathrm{~h} 5 \mathrm{bmu} 5 \mathrm{vad}$ \\
\hline $\begin{array}{l}\text { mRNA of Insulin-like growth fac- } \\
\text { tor } 1 \text { receptor/ Target of antisense } \\
\text { drug/ T } 64977\end{array}$ & $\begin{array}{l}\text { 1igr } 1 \text { jqh } 1 \mathrm{k} 3 \mathrm{a} \quad 1 \mathrm{~m} 7 \mathrm{n} \text { 1p4o } 2 \text { oj9 } 2 \mathrm{zm} 3 \text { 3d94 3f5p 3i81 3lvp 3lw0 3nw5 3nw6 3nw7 3o23 } \\
3 \mathrm{qqu} 4 \mathrm{~d} 2 \mathrm{r} 4 \mathrm{xss} 5 \text { fxq } 5 \text { fxr } 5 \text { fxs } 5 \mathrm{hzn}\end{array}$ \\
\hline
\end{tabular}




\begin{tabular}{|c|c|}
\hline $\begin{array}{l}\text { CAMP-specific } 3 ', 5^{\prime} \text {-cyclic phos- } \\
\text { phodiesterase } 4 \mathrm{~A} / \text { Phosphoric di- } \\
\text { ester hydrolases/ T61744 }\end{array}$ & $2 q y k 3 i 8 v 3 t v x$ \\
\hline $\begin{array}{l}\text { Retinoic acid receptor beta/ } \mathrm{Nu}- \\
\text { clear hormone receptor/ T61657 }\end{array}$ & 1hra 1 xap $4 \mathrm{dm} 64 \mathrm{dm} 84$ jyg 4 jyh 4 jyi 5 uan \\
\hline $\begin{array}{l}\text { Aldosterone synthase/ Oxidoreduc- } \\
\text { tases acting on paired donors/ } \\
\text { T59056 }\end{array}$ & $4 \mathrm{dvq} 4 \mathrm{fdh} 4 \mathrm{zgx}$ \\
\hline $\begin{array}{l}\text { Cytochrome P450 2D6/ Oxidore- } \\
\text { ductases acting on paired donors/ } \\
\text { T57392 }\end{array}$ & 2f9q 3qm4 3tbg 3tda 4 wnt 4 wnu 4 wnv 4 wnw 4 xry 4 xrz 5 tft 5 tfu \\
\hline $\begin{array}{l}\text { Interleukin-1receptor-associated } \\
\text { kinase-4/ Kinase/ T55398 }\end{array}$ & $\begin{array}{l}\text { 2nru 2nry 2o8y 2oib 2oic 2oid 3mop 4rmz 4u97 4u9a 4xs2 4y73 4yo6 4yp } 4 \text { ztl 4ztm 4ztn } \\
5 \mathrm{kx} 75 \mathrm{kx} 8 \text { 5t1s 5t1t 5uiq 5uir 5uis 5uit 5uiu 5w84 5w85 }\end{array}$ \\
\hline $\begin{array}{l}\text { Oxysterols receptor LXR-alpha/ } \\
\text { Nuclear hormone receptor/ T52297 }\end{array}$ & 1uhl 3ipq 3ips 3ipu 5avi 5avl 5hjs \\
\hline $\begin{array}{l}\text { Porphobilinogen deaminase/ Alkyl } \\
\text { aryl transferase/ T47466 }\end{array}$ & 3ecr 3eq 1 \\
\hline $\begin{array}{l}\text { Aldehyde dehydrogenase, mito- } \\
\text { chondrial/ Oxidoreductases acting } \\
\text { on aldehyde or oxo group of donors/ } \\
\text { T44282 }\end{array}$ & 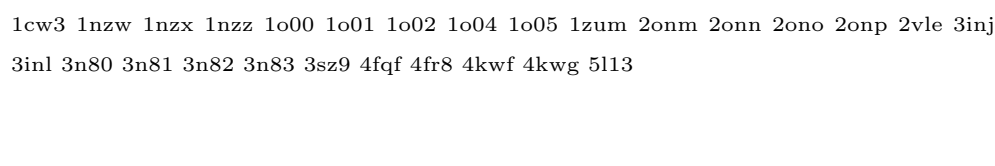 \\
\hline $\begin{array}{l}\text { mRNA of human IL-1 receptor- } \\
\text { associated kinase-4/ Kinase/ } \\
\text { T41766 }\end{array}$ & $\begin{array}{l}\text { 2nru 2nry 2o8y 2oib 2oic 2oid 3mop 4rmz 4u97 4u9a 4xs2 4y73 4yo6 4yp8 4ztl 4ztm 4ztn } \\
5 \mathrm{kx} 75 \mathrm{kx} 8 \text { 5t1s 5t1t 5uiq 5uir 5uis 5uit 5uiu 5w84 5w85 }\end{array}$ \\
\hline $\begin{array}{l}\text { mRNA of SYK/ Target of siRNA } \\
\text { drug/ T37952 }\end{array}$ & 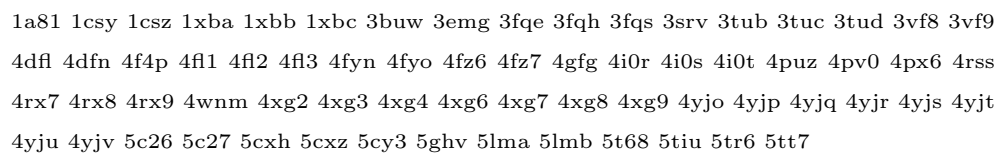 \\
\hline $\begin{array}{l}\text { Soluble epoxide hydrolase/ Ether- } \\
\text { hydrolase/ T } 35734\end{array}$ & 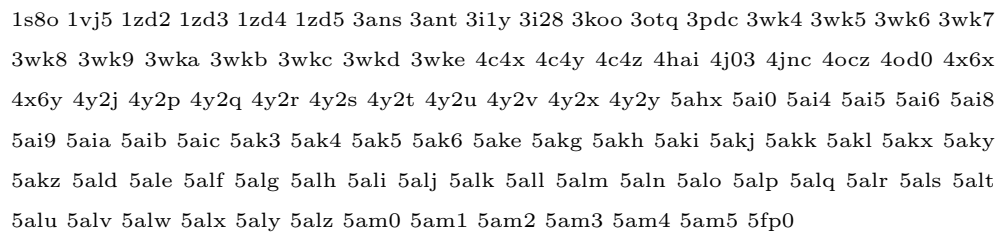 \\
\hline
\end{tabular}




\begin{tabular}{|c|c|}
\hline $\begin{array}{l}\text { HIF prolyl hydroxylase- } 2 / \text { Oxidore- } \\
\text { ductases acting on paired donors/ } \\
\text { T32880 }\end{array}$ & $\begin{array}{l}\text { 2g19 2g1m 2hbt 2hbu 2y33 2y34 3hqr 3hqu 3ouh 3oui 3ouj 4bqw } 4 \text { bqx } 4 \text { bqy } 4 \text { jzr } 4 \mathrm{kbz} \\
4 \text { uwd 5a3u 519b 519r 519v 5la9 5las 5lat 5lb6 5lbb 5lbc 5lbe 5lbf 5ox 5 5ox6 5v18 }\end{array}$ \\
\hline $\begin{array}{l}\text { Glutamyl aminopeptidase/ Pepti- } \\
\text { dase/ T31956 }\end{array}$ & $4 \mathrm{kx} 74 \mathrm{kx} 8 \quad 4 \mathrm{kx} 9 \quad 4 \mathrm{kxa} 4 \mathrm{kxb} 4 \mathrm{kxc} 4 \mathrm{kxd}$ \\
\hline $\begin{array}{l}\text { Glutathione reductase/ Oxidore- } \\
\text { ductases acting on a sulfur group of } \\
\text { donors/ T30803 }\end{array}$ & $\begin{array}{l}\text { 1alg 1bwc 1dnc 1gra } 1 \text { grb } 1 \text { gre } 1 \text { grf } 1 \text { grg } 1 \text { grh } 1 \text { grt } 1 \text { gsn } 1 \mathrm{k} 4 \mathrm{q} 1 \mathrm{xan} 2 \mathrm{aaq} 2 \mathrm{gh} 52 \mathrm{grt} 3 \mathrm{djg} \\
3 \mathrm{djj} 3 \mathrm{dk} 43 \mathrm{dk} 83 \mathrm{dk} 93 \mathrm{grs} 3 \mathrm{grt} 3 \mathrm{sqp} 4 \mathrm{gr} 14 \mathrm{grt} 5 \mathrm{grt}\end{array}$ \\
\hline $\begin{array}{l}\text { Serine/ threonine-protein kinase } \\
\text { Chk2/ T28713 }\end{array}$ & 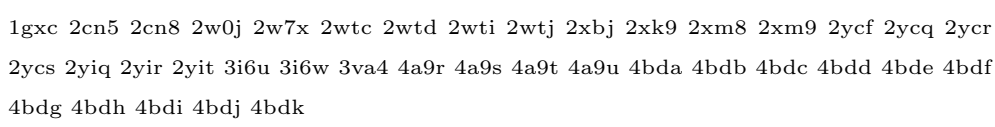 \\
\hline $\begin{array}{l}\text { Recombinant human angiotensin } \\
\text { converting enzyme 2/ Peptidase/ } \\
\text { T27889 }\end{array}$ & 1r42 1r4l 1xjp 2ajf 3d0g 3d0h 3d0i 3kbh 3sci 3scj 3sck 3scl \\
\hline $\begin{array}{l}\text { Nuclear receptor coactivator } 3 / \\
\text { Acyltransferase/ T2 } 26774\end{array}$ & $1 \mathrm{kbh} 313 \mathrm{x} 313 \mathrm{z}$ \\
\hline $\begin{array}{l}\text { Myeloperoxidase/ Peroxidases/ } \\
\text { T23471 }\end{array}$ & $\begin{array}{l}1 \mathrm{cxp} 1 \mathrm{~d} 2 \mathrm{v} 1 \mathrm{~d} 5 \mathrm{l} 1 \mathrm{~d} 7 \mathrm{w} 1 \mathrm{dnu} 1 \mathrm{dnw} 1 \mathrm{mhl} 1 \mathrm{myp} 3 \mathrm{f} 9 \mathrm{p} 3 \mathrm{zs} 03 \mathrm{zs} 14 \mathrm{c} 1 \mathrm{~m} 4 \mathrm{dl} 14 \mathrm{ejx} 5 \mathrm{fiw} 5 \mathrm{mfa} \\
5 \mathrm{uzu}\end{array}$ \\
\hline $\begin{array}{l}\text { mRNA of integrin beta } 3 / \text { Integrin/ } \\
\text { T23355 }\end{array}$ & 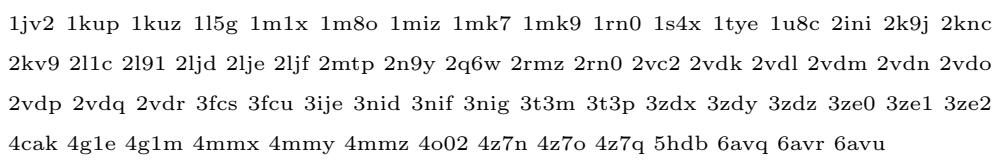 \\
\hline $\begin{array}{l}\text { mRNA of Bcl-x/ Bcl-2 family/ } \\
\text { T19923 }\end{array}$ & $\begin{array}{l}\text { 1bxl 1g5j 1lxl 1maz 1r2d 1r2e 1r2g 1r2h 1r2i 1ysg 1ysi 1ysn 2b48 2lp8 2lpc 2m03 2m04 } \\
\text { 2me8 2me9 2mej 2o1y 2o2m 2o2n 2p1l 2pon 2yj1 2yq6 2yq7 2yxj 3cva 3fdl 3fdm 3inq 3io8 } \\
\text { 3pl7 3qkd 3r85 3sp7 3spf 3wiz 3zk6 3zln 3zlo 3zlr 4a1u 4a1w 4aq3 4bpk 4c52 4c5d 4cin } \\
\text { 4ehr 4hnj 4ieh 4ppi 4qve 4qvf 4qvx 4tuh 4z9v 5agw 5agx 5b1z 5c3g 5fmj 5fmk 6bf2 }\end{array}$ \\
\hline
\end{tabular}


Heat shock protein HSP 90/ Heat shock protein/ T18477 1byq 1osf 1uy6 1uy7 1uy8 1uy9 1uyc 1uyd 1uye 1uyf 1uyg 1uyh 1uyi 1uyk 1uyl 1yc1 1yc3 1yc4 1yer 1yes 1yet 2bsm 2bt0 2bug 2byh 2byi 2bz5 2c2l 2ccs 2cct 2ccu 2fwy 2fwz 2 h55 2jjc 2k5b 2qf6 2qfo 2qg0 2qg2 2uwd 2vci 2vcj 2wi1 2wi2 2wi3 2wi4 2wi5 2wi6 2wi7 2xab $2 x d k 2 x d l$ xds $2 x d u 2 x d x$ 2xhr 2xht 2xhx 2xjg 2xjj 2xjx 2xk2 2ye2 2ye3 2ye4 2ye5 2ye6 2 ye7 2ye8 2ye9 2yea 2yeb 2yec 2yed 2yee 2yef 2yeg 2yeh 2yei 2yej 2yi0 2yi5 2yi6 2yi7 2yjw 2yjx 2yk2 2yk9 2ykb 2ykc 2yke 2yki 2ykj 3b24 3b25 3b26 3b27 3b28 3bm9 3bmy 3d0b 3eko 3ekr 3ft5 3ft8 3hek 3hhu 3hyy 3hyz 3hz1 3hz5 3inw 3inx 3k97 3k98 3k99 3mnr 3o0i 3ow6 3owb 3owd 3q6m 3q6n 3qdd 3qtf 3r4m 3r4n 3r4o 3r4p 3r91 3r92 3rkz 3rlp 3rlq 3rlr 3t0h 3t0z 3t10 3t1k 3t2s 3tuh 3vha 3vhc 3vhd 3wha 3wq9 4aif 4awo 4awp 4awq 4b7p 4bqg 4bqj 4cgq 4cgu 4cgv 4cgw 4cwf 4cwn 4cwo 4cwp 4cwq 4cwr 4cws 4cwt 4eeh $4 \mathrm{eft}$ 4efu 4egh 4egi 4egk 4fcp 4fcq 4fcr 4hy6 4jql 4l8z 4190419141934194 4lwe 4lwf 4lwg 4lwh 4lwi 4nh7 4nh8 4o04 4o05 4o07 4o09 4o0b 4r3m 4u93 4w7t 4xip 4xiq 4xir 4xit 4ykq 4ykr 4 ykt 4 yku 4ykw 4ykx 4yky 4ykz 5 cfo 5 fnc 5 fnd 5 fnf 5 ggz 5j2v 5lq9 5lr1 5lr7 5lrl 5lrz 5ls 1 $5 \mathrm{~m} 4 \mathrm{e} 5 \mathrm{~m} 4 \mathrm{~h} 5 \mathrm{t} 21$

1lwz 1zxm 1zxn 4fm9 4r1f 5gwk

DNA topoisomerase II alpha/ ATP hydrolyzing DNA topoisomerase/ T17048

Carbonic anhydrase XII/ Carbonoxygen lyases/ T16987

Oxysterols receptor LXR-beta/ Nuclear hormone receptor/ T13714

$73-\mathrm{kDa} / \mathrm{molecular}$

HSP73/ Heat shock protein/

3agy 3agz 3esk 3fzf 3fzh 3fzk 3fzl 3fzm 3ldq 3m3z 4h5n 4h5r 4h5t 4h5v 4h5w 4hwi 4kbq 5 aqf 5 aqg 5 aqh 5 aqi 5aqj 5aqk 5 aql 5 aqm 5 aqn 5 aqo 5 aqp 5 aqq 5 aqr 5 aqs 5 aqt 5 aqu 5 aqv

$\mathrm{T} 12817$

mRNA of Human mdm2/ Carbonnitrogen ligase/ T10937

1rv1 1t4e 1t4f 1ycr 1z1m 2axi 2c6a 2c6b 2f1y 2fop 2gv2 2hdp 2lzg 2m86 2mps 2ruh 2vje 2vjf 3eqs 3g03 3iux 3iwy 3jzk 3jzr 3jzs 3lbk 3lbl 3lnj 3lnz 3mqs 3tj2 3tpx 3tu1 3v3b 3vbg 3vzv 3w69 4dij 4ere 4erf 4hbm 4hfz 4hg7 4jv7 4jv9 4jve 4jvr 4jwr 4mdn 4mdq 4oas 4oba 4occ 4ode 4odf 4ogn 4ogt 4ogv 4oq3 4qo4 4qoc 4ud7 4ue1 4umn 4wt2 4xxb 4zfi 4zgk 4zyc 4 zyf 4 zyi 5 afg 5 c5a 5 hmh 5 hmi 5 hmk 5j7f 5j7g 5lav 5law 5lay 5laz 5ln2 5mnj 5swk 5 trf

mRNA of human Jun-N-terminal 3e7o 3 npc kinase-2 (JNK2)/ Kinase/ T06037 mRNA of ERBB3/ Kinase/ T04084

1m6b 219u 3kex 3lmg 3p11 4leo 4otw 4p59 4riw 4rix 4riy 5cus

Leukotriene A-4 hydrolase/ Etherhydrolase/ T03691

1gw6 1h19 1hs6 1sqm 2r59 2vj8 3b7r 3b7s 3b7t 3b7u 3cho 3chp 3chq 3chr 3chs 3fh5 3fh7 $3 \mathrm{fh} 8$ fhe $3 \mathrm{fts} 3 \mathrm{ftu} 3 \mathrm{ftv} 3 \mathrm{ftw} 3 \mathrm{ftx} 3 \mathrm{fty} 3 \mathrm{ftz} 3 \mathrm{fu} 03 \mathrm{fu} 33 \mathrm{fu} 5$ fu6 $3 \mathrm{fud} 3 \mathrm{fue} 3 \mathrm{fuf} 3 \mathrm{fuh} 3 \mathrm{fui}$ 3fuj 3fuk 3ful 3fum 3fun 3u9w 4dpr 4121 mkt 4ms6 4r7l 4rsy 4rvb 5aen 5bpp 5fwq 5n3w 5ni2 5ni4 5ni6 5nia 5nid 5nie 


\begin{tabular}{|c|c|}
\hline MMP-12/ Peptidase/ T03500 & $\begin{array}{l}\text { 1jiz 1jk3 1os2 1os9 1rmz 1ros 1utt 1utz 1y93 1ycm 1z3j 2hu6 2jxy 2k2g 2k9c 2krj 2mlr } \\
\text { 2mls 2n8r 2oxu 2oxw 2oxz 2poj 2w0d 2wo8 2wo9 2woa 2z2d 3ba0 3ehx 3ehy 3f15 3f16 } \\
\text { 3f17 3f18 3f19 3f1a 3lik 3lil 3lir 3ljg 3lk8 3lka 3n2u 3n2v 3nx7 3rts 3rtt 3ts4 3tsk 3uvc } \\
\text { 4efs 4gql 4gr0 4gr3 4gr8 4guy 4h30 4h49 4h76 4h84 4i03 4ijo 5cxa 5czm 5d2b 5d3c 5i0l } \\
\text { 5i2z 5i3m 5i43 5i4o 5l79 5l7f 5lab 5n5j 5n5k }\end{array}$ \\
\hline Aggrecanase/ Peptidase/ T98896 & 2rjq 3b8z 3hy7 3hy9 3hyg 3ljt \\
\hline $\begin{array}{l}\text { PAK-4 protein kinase/ Kinase/ } \\
\text { T98293 }\end{array}$ & $\begin{array}{l}\text { 2bva 2cdz 2j0i 2ov2 2q0n 2x4z 4app 4fie 4fif 4fig } 4 \text { fih } 4 \text { fii } 4 \text { fij } 4 \text { jdh } 4 \text { jdi } 4 \text { jdj } 4 \text { jdk } 41674 \text { njd } \\
4 \mathrm{o} 0 \mathrm{v} 4 \mathrm{o} 0 \mathrm{x} 4 \mathrm{o} 0 \mathrm{y} 4 \mathrm{xbr} 4 \mathrm{xbu} 5 \mathrm{bms} 5 \mathrm{i} 0 \mathrm{~b} 5 \text { ved } 5 \text { vee } 5 \text { vef }\end{array}$ \\
\hline $\begin{array}{l}\text { Transforming protein RhoA/ Small } \\
\text { GTPase superfamily/ T } 96736\end{array}$ & 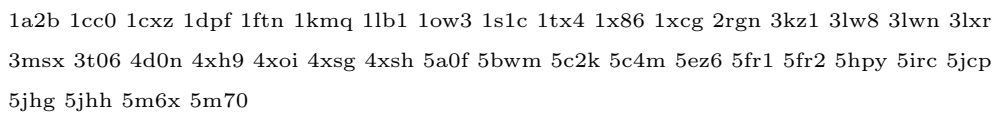 \\
\hline $\begin{array}{l}\text { Branched chain amino acid- } \\
\text { dependent aminotransferase/ } \\
\text { Transferases of nitrogenous groups/ } \\
\text { T94788 }\end{array}$ & 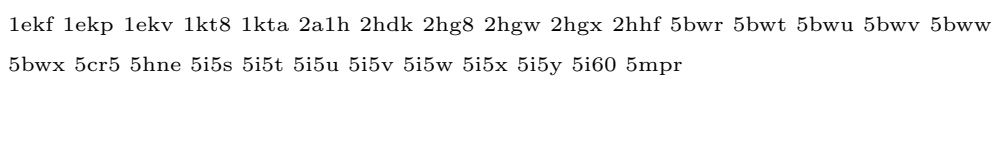 \\
\hline $\begin{array}{l}\text { DOT1L/ Methyltransferase super- } \\
\text { family/ T } 87686\end{array}$ & $\begin{array}{l}\text { 1nw3 2mv7 3qow 3qox 3sr4 3sx0 3uwp 4ek9 4ekg 4eki 4eqz 4er0 4er3 4er5 4er6 4er7 4hra } \\
4 \text { wvl 5drt 5dry 5dsx 5dt2 } 5 \mathrm{dtm} 5 \mathrm{dtq} 5 \mathrm{dtr} 5 \text { juw } 5 \mathrm{mvs} 5 \mathrm{mw} 35 \mathrm{mw} 4\end{array}$ \\
\hline $\begin{array}{l}\text { Folate receptor alpha/ Folate recep- } \\
\text { tor/ T83386 }\end{array}$ & $4 \mathrm{~km} 64 \mathrm{~km} 74 \mathrm{kmx}$ 4lrh $5 \mathrm{izq}$ \\
\hline $\begin{array}{l}\text { mRNA of p53/ Target of siRNA } \\
\text { drug/ T } 82051\end{array}$ & 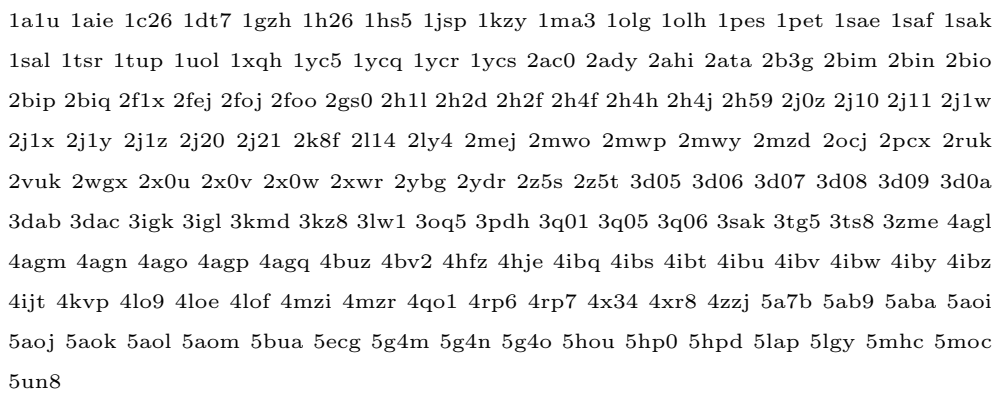 \\
\hline $\begin{array}{l}\text { mRNA of Caspase-2/ Target of } \\
\text { siRNA drug/ T } 81103\end{array}$ & 1pyo 2p2c 3r5j 3r6g 3r61 3r7b 3r7n 3r7s 3rjm \\
\hline $\begin{array}{l}\text { Phenylalanine hydroxylase/ Ox- } \\
\text { idoreductases acting on paired } \\
\text { donors/ T76213 }\end{array}$ & $\begin{array}{l}\text { 1dmw 1j8t 1j8u 1kw0 1lrm 1mmk 1mmt 1pah 1tdw 1tg2 2pah 3pah 4anp 4pah 5fii 5pah } \\
6 \mathrm{pah}\end{array}$ \\
\hline $\begin{array}{l}\text { FK506-binding protein } 1 \mathrm{~A} / \mathrm{Cis}- \\
\text { trans-isomerases/ T76059 }\end{array}$ & 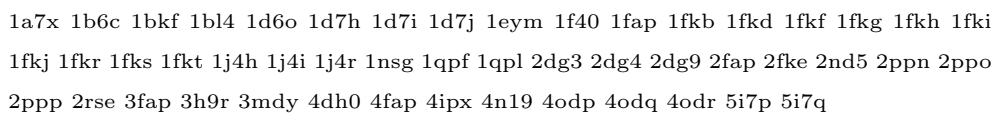 \\
\hline
\end{tabular}




\begin{tabular}{|c|c|}
\hline $\begin{array}{l}\text { Methionine aminopeptidase } 2 / \text { Pep- } \\
\text { tidase/ T75596 }\end{array}$ & $\begin{array}{l}\text { 1b59 1b6a 1bn5 1boa 1kq0 1kq9 1qzy } 1 \text { r58 } 1 \text { r5g } 1 \text { r5h } 1 \text { yw7 } 1 \text { yw } 8 \text { 1yw9 2adu 2ea2 2ea } 4 \\
2 \text { ga2 2oaz } 5 \text { cls } 5 \text { d6e } 5 \text { d6f } 5 \text { jfr } 5 \text { jhu } 5 \text { ji6 } 5 \text { lyw } 5 \text { lyx }\end{array}$ \\
\hline Lethal factor/ Peptidase/ T72335 & $\begin{array}{l}\text { 1j7n 1jky 1pwp 1pwq 1pwu 1pwv 1pww 1yqy } 1 \text { zxv } 210 \mathrm{r} 3 \mathrm{kwv} 4 \mathrm{dv} 8 \text { pkq } 4 \mathrm{pkr} 4 \mathrm{pks} 4 \mathrm{pkt} \\
4 \mathrm{pku} 4 \mathrm{pkv} 4 \mathrm{pkw} 4 \mathrm{wf} 64 \mathrm{xm} 64 \mathrm{xm} 74 \mathrm{xm} 85 \mathrm{~d} 1 \mathrm{~s} 5 \mathrm{~d} 1 \mathrm{t} 5 \mathrm{~d} 1 \mathrm{u}\end{array}$ \\
\hline $\begin{array}{l}\text { Orphan nuclear receptor NR1I3/ } \\
\text { Nuclear hormone receptor/ T69506 }\end{array}$ & $1 \mathrm{xv} 91 \mathrm{xvp}$ \\
\hline $\begin{array}{l}\text { Mitogen-activated protein kinase 9/ } \\
\text { Kinase/ T69375 }\end{array}$ & 3e7o 3 npc \\
\hline $\begin{array}{l}\text { Zap70 tyrosine kinase/ Kinase/ } \\
\text { T64553 }\end{array}$ & 1fbv $1 \mathrm{~m} 611 \mathrm{u} 592 \mathrm{cbl} 2 \mathrm{oq} 12 \mathrm{ozo} 2 \mathrm{y} 1 \mathrm{n} 3 \mathrm{zni} 4 \mathrm{a} 4 \mathrm{~b} 4 \mathrm{a} 4 \mathrm{c} 4 \mathrm{k} 2 \mathrm{r} 4 \mathrm{xz} 04 \mathrm{xz} 1$ \\
\hline $\begin{array}{l}\text { Serum albumin/ Serum albumin } \\
\text { family/ T63068 }\end{array}$ & $\begin{array}{l}\text { 1ao6 1bj5 1bke } 1 \text { bm0 } 1 \text { e78 1e7a 1e7b 1e7c 1e7e 1e7f 1e7g 1e7h 1e7i 1gni 1gnj 1h9z 1ha2 } \\
\text { 1hk1 1hk2 1hk3 1hk4 1hk5 1n5u 1o9x 1tfo 1uor 1ysx 2bx8 2bxa 2bxb 2bxc 2bxd 2bxe } \\
\text { 2bxf 2bxg 2bxh 2bxi 2bxk 2bxl 2bxm 2bxn 2bxo 2bxp 2bxq 2esg 2i2z 2i30 2n0x 2vdb 2vue } \\
\text { 2vuf 2xsi 2xvq 2xvu 2xvv 2xvw 2xw0 2xw1 2ydf 3a73 3b9l 3b9m 3cx9 3jqz 3jry 3lu6 3lu7 } \\
\text { 3lu8 3sqj 3tdl 3uiv 4bke 4e99 4emx 4g03 4g04 4hgk 4hgm 4iw1 4iw2 4k2c 4k71 4l8u 419k } \\
\text { 419q 4la0 4lb2 4lb9 4n0f 4n0u 4s1y 4z69 5fuo 5gix 5giy 5id7 5ifo 5ijf 5ujb 5x52 }\end{array}$ \\
\hline $\begin{array}{l}\text { Beta-adrenergic receptor kinase } 1 / \\
\text { Kinase/ T62548 }\end{array}$ & 1bak 3cik 3krw $3 \mathrm{krx} 3 \mathrm{v} 5 \mathrm{w} 4 \mathrm{mk0} 4 \mathrm{pnk} 5$ he1 5 ukk 5 ukl 5uuu 5 uvc \\
\hline $\begin{array}{l}\text { Sorbitoldehydrogenase/ Short- } \\
\text { chain dehydrogenases reductases/ } \\
\text { T59445 }\end{array}$ & $1 \mathrm{pl} 61 \mathrm{pl} 71 \mathrm{pl} 8$ \\
\hline Cyclin-A2/ T58470 & 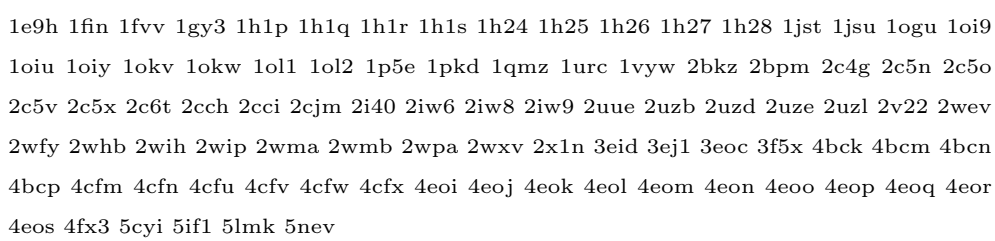 \\
\hline
\end{tabular}



$1 \mathrm{rvl} 1 \mathrm{rvm} 1 \mathrm{rvn} 1 \mathrm{rvo} 1 \mathrm{rvp} 1 \mathrm{rvq} 1 \mathrm{rvr} 1 \mathrm{~s} 6 \mathrm{p}$ 1s6q 1s9e 1s9g 1sbg 1suq 1sv5 1t03 1t05 1t7k 1tv6 1tvr 1uwb 1w5v 1w5w 1w5x 1w5y 1yt9 1zp8 1zpa 1zsf 1zsr 2aqu 2b5j 2b6a 2ban 2bbb 2be2 2exf 2g69 2hb3 2hmi 2hnz 2hs1 2hs2 2i4d 2i4u 2i4v 2i4w 2i4x 2i5j 2iaj 2ic3 2idw 2ieo 2jzw 214521462141 2uxz 2uy0 2vg5 2vg6 2vg7 2x4u 2ykm 2ykn 2zd1 2ze2 3avi 3bgr 3dlk 3gga 3ggv 3ggx 3hvt 3ig1 3irx 3is9 3isn 3ith 3jsm 3jyt 3k2p 3k4v 3kle 3klf 3klg 3klh 3kli 3ndt 3nu3 3nu4 3nu5 3nu6 3nu9 3nuj 3nuo 3ok9 3psu 3qaa 3qlh 3qo9 3tkg 3tkw 3t19 3tlh 3v4i 3v6d 3v81 3zps 3zpt 3zpu 4coe 4cp7 4cpq 4cpr 4cps 4cpt 4cpu 4cpw 4cpx 4dg1 4g1q 4g8g 4g8i 4g9d 4g9f 4h4m 4h4o 4i2p 4i2q 4icl 4id5 4idk 4ifv 4ify 4ig0 4ig3 4kfb 4kko 4ko0 4lsl 4lsn 4mfb 4o44 4o4g 4ojr 4pqu 4puo 4pwd 4q0b 4qag 4r5p 4rw4 4rw6 4rw7 4rw8 4rw9 4u8w 4we1 4ye3 4yhq 4zip 4zls 5agz 5ah6 5ah7 5ah8 5ah9 5aha 5ahb 5ahc 5 bry 5bs4 5c24 5c25 5c42 5cym 5cyq 5d3g 5fdl 5hbm 5hlf 5hp1 5hro 5i3u 5i42 5j1e 5jfp 5 jfu 5 jg1 5t6z 5t70 5tep 5ter 5tuq 5tw3 5txl 5txm 5txn 5txo 5txp 5ult 5uov 5upz 5uv5 $5 \mathrm{v} 515 \mathrm{v} 5 \mathrm{~m} 5 \mathrm{vqq} 5 \mathrm{vqr} 5 \mathrm{vqs} 5 \mathrm{vqt} 5 \mathrm{vqu} 5 \mathrm{vqv} 5 \mathrm{vqw} 5 \mathrm{vqx} 5 \mathrm{vqy} 5 \mathrm{vqz}$

ALK tyrosine kinase receptor/ Kinase/ T56418

Farnesyl pyrophosphate synthase/

Alkyl aryl transferase/ T52373

\begin{tabular}{l} 
\\
\hline Proto-oncogene pim-1/ Kinase/ \\
T50594
\end{tabular}

2kup 2kuq 2xb7 2xba 2xp2 2yfx 2yhv 2yjr 2yjs 2ys5 2yt2 3aox 319p 3lcs 3lct 4anl 4anq $4 \mathrm{ans} 4 \mathrm{ccb} 4 \mathrm{ccu} 4 \mathrm{cd0} 4 \mathrm{cli} 4 \mathrm{clj} 4 \mathrm{cmo} 4 \mathrm{cmt} 4 \mathrm{cmu} 4 \mathrm{cnh} 4 \mathrm{ctb} 4 \mathrm{ctc} 4 \mathrm{dce} 4 \mathrm{fnw} 4 \mathrm{fnx} 4 \mathrm{fny} 4 \mathrm{fnz}$ 4 fob 4 foc 4 fod 4 joa 4 mkc 4 tt7 4 z55 5a9u 5 aa 8 aa9 5 aaa 5 aab 5 aac 5 fto 5 ftq 5 imx 5 iug 5iuh 5iui $5 \mathrm{j} 7 \mathrm{~h} 5 \mathrm{kz0}$

1yq7 1yv5 1zw5 2f7m 2f89 2f8c 2f8z 2f92 2f94 2f9k 2opm 2opn 2qis 2rah 2vf6 3b7l 3cp6 3n1v 3n1w 3n31 3n45 3n46 3n49 3n5h 3n5j 3n6k 3rye 3s4j 4dem 4ga3 4h5c 4h5d 4h5e 4jvj $4 \mathrm{kfa} 4 \mathrm{kpd} 4 \mathrm{kpj} 4 \mathrm{kq} 5$ 4kqs 4kqu 412x 4lfv 4lpg 4lph 4n1z 4n9u 4nfi 4nfj 4nfk 4ng6 4nke 4 nkf 4nua 4ogu 4p0v 4p0w 4p0x 4pvx 4pvy 4q23 4qpf 4qxs 4rxa 4xqr 4xqs 4xqt 5cg5 5cg6 5 dgm 5 dgn 5 dgs 5 diq 5 djp 5 djr 5 djv 5 ja0 5 juz 5 jv0 5 jv 15 jv2 $5 \mathrm{ksx}$

1xqz 1xr1 1xws 1yhs 1yi3 1yi4 1ywv 1yxs 1yxt 1yxu 1yxv 1yxx 2bik 2bil 2bzh 2bzi 2bzj 2bzk 2c3i 2j2i 2o3p 2063 2o64 2o65 2obj 2oi4 2xix 2xiy 2xiz 2xj0 2xj1 2xj2 3a99 3bgp 3bgq 3bgz 3bwf 3c4e 3cxw 3cy2 3cy3 3dcv 3f2a 3jpv 3jxw 3jy0 3jya 3ma3 3qf9 3r00 3r01 3r02 3r04 3t9i 3uix 3umw 3umx 3vbq 3vbt 3vbv 3vbw 3vbx 3vby 3vc4 3we8 4a7c 4alu 4alv 4alw 4as0 4bzn 4bzo 4dtk 4enx 4eny 4gw8 4i41 4iaa 4jx3 4jx7 4k0y 4k18 4k1b 4ll5 $4 \operatorname{lm} 541 \mathrm{mu} 4 \mathrm{mbi} 4 \mathrm{mbl}$ 4med 4mta 4n6y 4n6z 4n70 4rbl 4rc2 4rc3 4rc4 4rpv 4ty1 4wrs 4wsy 4 wt 6 4xh6 4xhk 5c1q 5dgz 5 dhj 5 dia 5 dwr 5 eol 5 iis 5 ipj 5 kcx 5 kgd 5 kge 5 kgg 5 kgi $5 \mathrm{kgk} 5 \mathrm{kzi} 5 \mathrm{o} 115 \mathrm{o} 125 \mathrm{o} 135$ tel 5 tex 5 toe 5 tur $5 \mathrm{v} 82$

mRNA of Eukaryotic translation initiation factor $4 \mathrm{E} / \mathrm{T} 48614$

1ipb 1ipc 1wkw 2gpq 2v8w 2v8x 2v8y 2w97 3am7 3tf2 3u7x 4aza 4bea 4dt6 4dum 4tpw 4 tqb 4tqc 4ued 5ehc 5ei3 5eir 5ekv 5gw6 5t46

1g5m 1gjh 1ysw 2o21 2o22 2o2f 2w31 2xa0 4aq3 4ieh 4lvt 4lxd 4man 5agw 5agx 5fcg 5jsn

2wjw 2wjx 2xhd 3r7x 3rn8 3rnn 3ua 8 hh8s
mRNA of BCL-2/ Target of antisense drug/ T47387

1

Glutamate receptor 2/ Glutamategated ion channel/ T42392

2wjw 2wjx 2xhd 3r7x 3rn8 3rnn 3 ua 8 h 8 s




\begin{tabular}{|c|c|}
\hline $\begin{array}{l}\text { 5'-methylthioadenosine phosphory- } \\
\text { lase/ Pentosyltransferase/ T40787 }\end{array}$ & $1 \mathrm{cb} 01 \mathrm{cg} 61 \mathrm{k} 27$ 1sd1 $1 \mathrm{sd} 23 \ln 53 \mathrm{ozc} 3 \mathrm{ozd} 3 \mathrm{oze} 5$ eub 5 tc5 5 tc6 5 tc $75 \mathrm{tc} 8$ \\
\hline $\begin{array}{l}\text { Biliverdin reductase A/ Oxidore- } \\
\text { ductases acting on } \mathrm{CH}-\mathrm{CH} \text { group of } \\
\text { donors/ T37046 }\end{array}$ & $2 \mathrm{~h} 63$ \\
\hline $\begin{array}{l}\text { Cholesterol 24-hydroxylase/ Ox- } \\
\text { idoreductases acting on paired } \\
\text { donors/ T33641 }\end{array}$ & 2q9f 2q9g 3mdm 3mdr 3mdt 3mdv 4 enh 4 fia $4 j 14$ \\
\hline $\begin{array}{l}\text { mRNA of Caspase } 8 / \text { Peptidase/ } \\
\text { T28705 }\end{array}$ & $\begin{array}{l}\text { 1f9e 1i4e 1qdu 1qtn 2c2z 2fun } 2 \mathrm{k} 7 \mathrm{z} 2 \mathrm{y} 113 \mathrm{~h} 113 \mathrm{kjn} 3 \mathrm{kjq} 4 \mathrm{jj} 74 \mathrm{prz} 4 \mathrm{ps} 14 \mathrm{zbw} 5 \mathrm{~h} 315 \mathrm{~h} 33 \\
5 \text { jqe } 5108\end{array}$ \\
\hline $\begin{array}{l}\text { Kinesin-like protein } \quad \text { KIF11/ } \\
\text { Kinesin-like protein family/ T28484 }\end{array}$ & 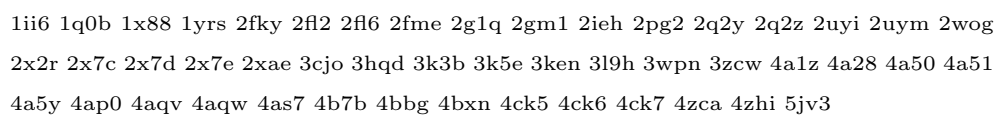 \\
\hline $\begin{array}{l}\text { mRNA of Beta } 2 \text { adrenoceptor/ } \\
\text { Target of siRNA drug/ T24555 }\end{array}$ & $\begin{array}{l}\text { 1gq4 2r4r 2r4s 2rh1 3d4s 3kj6 3ny8 3ny9 3nya 3p0g 3pds 3sn6 4gbr 4lde 4ldl 4ldo 4qkx } \\
5 \mathrm{~d} 5 \mathrm{a} 5 \mathrm{~d} 5 \mathrm{~b} 5 \mathrm{~d} 615 \mathrm{jqh} 5 \mathrm{x} 7 \mathrm{~d}\end{array}$ \\
\hline $\begin{array}{l}\text { Monoglyceride lipase/ Carboxylic } \\
\text { ester hydrolase/ T18664 }\end{array}$ & 3hju 3jw8 3jwe 3pe6 4uuq \\
\hline $\begin{array}{l}\text { Peptidyl-prolyl cis-trans isomerase } \\
\text { NIMA-interacting } 1 / \text { Cis-trans- } \\
\text { isomerases/ T16308 }\end{array}$ & 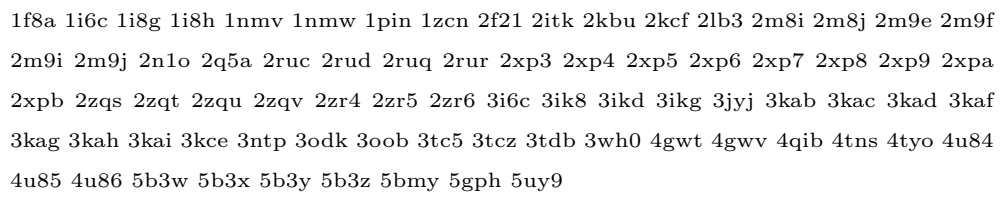 \\
\hline $\begin{array}{l}\text { mRNA of Factor XI/ Target of an- } \\
\text { tisense drug/ T15803 }\end{array}$ & $\begin{array}{l}\text { 1xx9 1xxd 1xxf 1zhm 1zhp 1zhr 1zjd 1zlr 1zmj 1zml 1zmn 1zom 1zpb 1zpc 1zpz 1zrk 1zsj } \\
\text { 1zsk 1zsl 1ztj 1ztk 1ztl 2f83 2fda 2j8j 2j81 3bg8 3sor 3sos 4cr5 4cr9 4cra 4crb 4crc 4crd } \\
4 \text { cre 4crf 4crg 4d76 4d7f 4d7g 4na7 4na8 4ty6 4ty7 4wxi 4x6m 4x6n 4x6o 4x6p 4y8x 4y8y } \\
\text { 4y8z 5e2o 5e2p 5eod 5eok 5exl 5exm 5exn 5i25 5q0d 5q0e 5q0f 5q0g 5q0h 5qck 5qcl 5qcm } \\
\text { 5qcn 5tks 5tkt 5tku 5wb6 }\end{array}$ \\
\hline
\end{tabular}




\begin{tabular}{|c|c|}
\hline $\begin{array}{l}\text { Induced myeloid leukemia cell dif- } \\
\text { ferentiation protein Mcl-1/ Bcl-2 } \\
\text { family/ T14912 }\end{array}$ & $\begin{array}{l}\text { 2kbw } 2 \mathrm{mhs} 2 \mathrm{nl} 92 \mathrm{nla} 2 \mathrm{pqk} 3 \mathrm{~d} 7 \mathrm{v} 3 \mathrm{io} 93 \mathrm{kj} 03 \mathrm{kj} 13 \mathrm{kj} 23 \mathrm{kz} 03 \mathrm{mk} 83 \mathrm{pk} 13 \mathrm{twu} 3 \mathrm{wix} 3 \mathrm{wiy} 4 \mathrm{bpi} \\
4 \mathrm{bpj} 4 \mathrm{hw} 24 \mathrm{hw} 34 \mathrm{hw} 44 \mathrm{oq} 54 \mathrm{oq} 64 \mathrm{wgi} 4 \mathrm{wmr} 4 \mathrm{wms} 4 \mathrm{wmt} 4 \mathrm{wmu} 4 \mathrm{wmv} 4 \mathrm{wmw} 4 \mathrm{wmx} 4 \mathrm{zbf} \\
4 \mathrm{zbi} 5 \mathrm{c} 3 \mathrm{f} 5 \mathrm{c} 6 \mathrm{~h} 5 \mathrm{fc} 45 \mathrm{fdo} 5 \mathrm{fdr} 5 \mathrm{iez} 5 \mathrm{if} 45 \mathrm{jsb} 5 \mathrm{ku} 95 \mathrm{lof} 5 \mathrm{mes} 5 \mathrm{mev} 5 \mathrm{uum} 5 \mathrm{vkc} 6 \mathrm{~b} 4 \mathrm{l} 6 \mathrm{bu}\end{array}$ \\
\hline Troponin C/ Troponin/ T12966 & 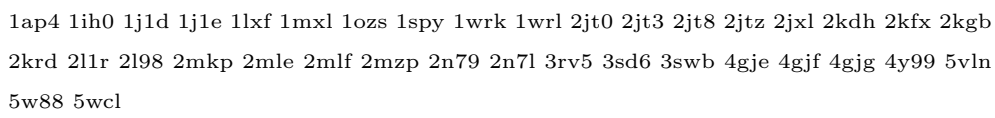 \\
\hline CD38/ Glycosylases/ T10877 & $\begin{array}{l}\text { 1yh3 1zvm 2ef1 2hct 2i65 2i66 2i67 2o3q 2o3r 2o3s 2o3t 2o3u 2pgj 2pgl 3dzf 3dzg 3dzh } \\
\text { 3dzi 3dzj 3dzk 3f6y 3i9m 3i9n 3ofs 3raj 3rok 3rom 3rop 3roq 3u4h 3u4i 4cmh } 4 \text { f45 } 4 \text { f46 } \\
4 \text { ogw 4tmf 4xjs 4xjt 5f1k 5f1o } 5 \text { f21 }\end{array}$ \\
\hline $\begin{array}{l}\text { Fatty acid-binding protein, } \\
\text { adipocyte/ Calycin family/ T07217 }\end{array}$ & $\begin{array}{l}\text { 1tou 1tow } 2 \mathrm{hnx} 2 \mathrm{nnq} 3 \mathrm{fr} 23 \mathrm{fr} 43 \mathrm{fr} 53 \mathrm{p} 6 \mathrm{c} 3 \mathrm{p} 6 \mathrm{~d} 3 \mathrm{p} 6 \mathrm{e} 3 \mathrm{p} 6 \mathrm{f} 3 \mathrm{p} 6 \mathrm{~g} 3 \mathrm{p} 6 \mathrm{~h} 3 \mathrm{q} 61 \text { 3rzy } 4 \mathrm{nns} 4 \mathrm{nnt} \\
5 \mathrm{~d} 455 \mathrm{~d} 475 \mathrm{~d} 485 \mathrm{~d} 4 \mathrm{a} 5 \mathrm{edb} 5 \mathrm{edc} 5 \mathrm{hz} 65 \mathrm{hz} 8\end{array}$ \\
\hline $\begin{array}{l}\text { mRNA of CYP3A4/ Target of anti- } \\
\text { sense drug/ T90391 }\end{array}$ & $\begin{array}{l}\text { 1tqn 1w0e 1w0f 1w0g 2j0d 2v0m 3nxu 3tjs 3ua1 4d6z 4d75 4d78 4d7d 4i3q 4i4g 4i4h 4k9t } \\
4 \mathrm{k} 9 \mathrm{u} 4 \mathrm{k} 9 \mathrm{v} 4 \mathrm{k} 9 \mathrm{w} 4 \mathrm{k} 9 \mathrm{x} 4 \mathrm{ny} 45 \mathrm{a} 1 \mathrm{p} 5 \mathrm{a} 1 \mathrm{r} 5 \mathrm{~g} 5 \mathrm{j} 5 \mathrm{te} 85 \mathrm{vc0} 5 \mathrm{vcc} 5 \mathrm{vcd} 5 \mathrm{vce} 5 \mathrm{vcg}\end{array}$ \\
\hline $\begin{array}{l}\text { Dihydrofolate reductase/ Oxidore- } \\
\text { ductases acting on } \mathrm{CH}-\mathrm{NH} \text { group of } \\
\text { donors/ T89515 }\end{array}$ & $3 g 5 k 3 g 5 p$ \\
\hline $\begin{array}{l}\text { Ras-related C3 botulinum toxin } \\
\text { substrate } 1 / \text { Small GTPase super- } \\
\text { family/ T } 88752\end{array}$ & 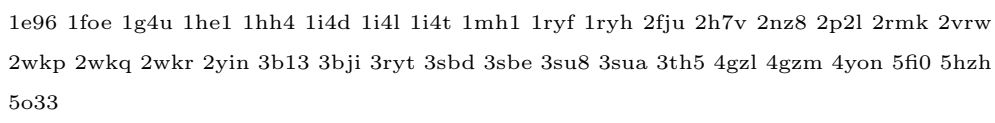 \\
\hline $\begin{array}{l}\text { Bromodomain-containing protein } \\
\text { 2/ Bromodomain/ T86399 }\end{array}$ & $\begin{array}{l}\text { 1x0j 2dvq 2dvr 2dvs 2dvv 2e3k 2g4a 2ydw 2yek 3aqa 3oni 4a9e 4a9f 4a9h 4a9i 4a9j 4a9m } \\
\text { 4a9n 4a9o 4akn 4alg 4alh 4j1p 4mr5 4mr6 4qeu 4qev 4qew 4uyf 4uyg 4uyh 5bt5 5dfb 5dfc } \\
5 \text { dfd 5dw1 5ek9 5hel 5hem 5hen 5hfq 5ibn 5ig6 5u5s 5uew 5xhe 5xhk }\end{array}$ \\
\hline $\begin{array}{l}\text { Phosphodiesterase 10A (PDE10A)/ } \\
\text { Phosphoric diester hydrolases/ } \\
\text { T84133 }\end{array}$ & 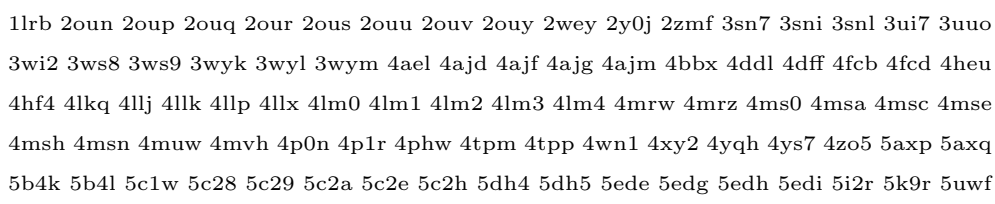 \\
\hline $\begin{array}{l}\text { Wiskott-Aldrich syndrome protein/ } \\
\text { T83585 }\end{array}$ & 1cee 1 ej5 1 t $842 \mathrm{a} 3 \mathrm{z} 2 \mathrm{k} 42$ 2ot0 \\
\hline $\begin{array}{l}\text { Pregnane X receptor/ Zinc-finger/ } \\
\text { T82702 }\end{array}$ & $\begin{array}{l}\text { 1ilg } 1 \text { ilh } 1 \mathrm{~m} 13 \text { 1nrl 1skx 2o9i 2qnv 3ctb 3hvl 3r8d 4j5w 4j5x 4ny9 4s0s 4s0t 4x1f 4x1g } \\
\text { 4xao 4xhd 5a86 5x0r }\end{array}$ \\
\hline $\begin{array}{l}\text { Endoplasmin/ Heat shock protein/ } \\
\text { T81311 }\end{array}$ & 4 nh9 \\
\hline
\end{tabular}




\begin{tabular}{|c|c|}
\hline $\begin{array}{l}\text { Enoyl-[acyl-carrier-protein] reduc- } \\
\text { tase }[\mathrm{NADH}] / \text { Oxidoreductases act- } \\
\text { ing on } \mathrm{CH}-\mathrm{CH} \text { group of donors/ } \\
\text { T79068 }\end{array}$ & 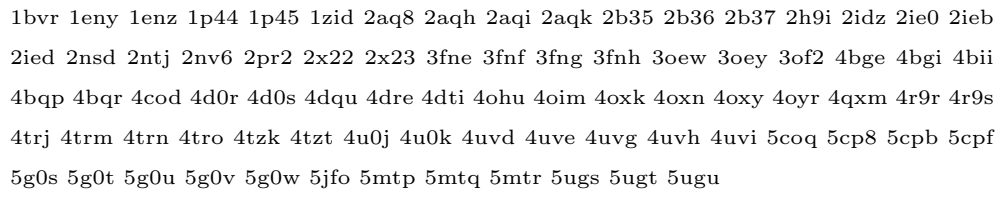 \\
\hline $\begin{array}{l}\text { mRNA of Aurora B/ Kinase/ } \\
\text { T77764 }\end{array}$ & $4 \mathrm{af} 3$ \\
\hline $\begin{array}{l}\text { Beta-ketoacyl-ACP synthase III/ } \\
\text { Acyltransferase/ T72444 }\end{array}$ & 1ebl 1 hn9 1 hnd 1 hnh 1 hnj $1 \mathrm{hnk} 1 \mathrm{mzs} 2$ eft 2 gyo $3 \mathrm{il} 9$ 4z 8 d 5 bnm 5 bnr 5 bns \\
\hline $\begin{array}{l}\text { Isocitrate dehydrogenase [NADP] } \\
\text { cytoplasmic/ Short-chain dehydro- } \\
\text { genases reductases/ T69563 }\end{array}$ & $\begin{array}{l}\text { 1t09 1t01 3inm 3map 3mar 3mas 4i3k 4i3l 4kzo } 4103410441064 \text { umx } 4 \text { umy } 4 \text { xrx } 4 \text { xs } 35 \text { de } 1 \\
\text { 5gir 5k10 5k11 } 51575158 \text { 5lge 5sun 5svf 5tqh 5yfm 5yfn 6b0z }\end{array}$ \\
\hline $\begin{array}{l}\text { Activated CDC42 kinase 1/ Kinase/ } \\
\text { T63220 }\end{array}$ & $1 \mathrm{cf} 41 \mathrm{u} 461 \mathrm{u} 4 \mathrm{~d} 1 \mathrm{u} 54$ 3eqp 3 eqr 4 ewh 4 hzr 4 hzs 4 id 7 \\
\hline $\begin{array}{l}\text { Deoxyhypusine synthase/ Alkyl } \\
\text { aryl transferase/ T62265 }\end{array}$ & $1 \mathrm{dhs} 1 \mathrm{rlz} 1 \mathrm{roz} 1 \mathrm{rqd}$ \\
\hline $\begin{array}{l}\text { Ornithine aminotransferase, mito- } \\
\text { chondrial/ Transferases of nitroge- } \\
\text { nous groups/ T } 62073\end{array}$ & 1 gbn 1 oat 2 byj 2 byl 2can 2 oat 5 vwo \\
\hline Polo-like kinase 2/ Kinase/ T59654 & $4 \mathrm{i} 5 \mathrm{~m} \mathrm{4i5p} \mathrm{4i6b} \mathrm{4i6f} \mathrm{4i6h} \mathrm{4rs6} \mathrm{4xb0}$ \\
\hline $\begin{array}{l}\text { mRNA of PLK1/ Target of siRNA } \\
\text { drug/ T59273 }\end{array}$ & $\begin{array}{l}\text { 1q4k 1q4o 1umw 2ogq 2ojx 2ou7 2owb 2rku 2v5q 2yac 3bzi 3c5l 3fc2 3fvh 3hih 3hik 3kb7 } \\
\text { 3p2w 3p2z 3p34 3p35 3p36 3p37 3q1i 3rq7 3thb 4a4l 4a4o 4dfw 4e67 4e9c 4e9d 4h5x 4h71 } \\
4 \text { hab 4hco 4hy2 4j52 4j53 4lkl 4lkm 4o56 4o6w 4o9w 4rcp 4whh 4whk 4whl 4x9r 4x9v } \\
4 \text { x9w 5j19 5ta6 5ta8 }\end{array}$ \\
\hline
\end{tabular}




\begin{tabular}{|c|c|}
\hline $\begin{array}{l}\text { Estradiol } 17 \text { beta-dehydrogenase } 4 / \\
\text { Short-chain dehydrogenases reduc- } \\
\text { tases/ T59230 }\end{array}$ & 1ikt 1s9c 1zbq \\
\hline $\begin{array}{l}\text { Glycoprotein IIb/ IIIa receptor/ } \\
\text { T50688 }\end{array}$ & 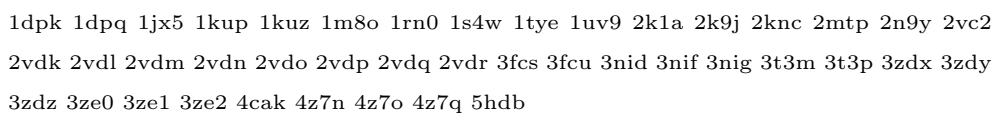 \\
\hline $\begin{array}{l}\text { Nonstructural protein NS3/ Pepti- } \\
\text { dase/ T40492 }\end{array}$ & 1rtl 2a4g 2gvf 3eyd 3hkw 3kn2 3qgh 3qgi 3rc4 3rc5 3su4 \\
\hline Cathepsin A/ Peptidase/ T37154 & 1ivy $3 \mathrm{bp} 43 \mathrm{bp} 73 \mathrm{bxn} 4 \mathrm{az} 0$ 4az3 4 ci9 4 cia 4 cib $4 \mathrm{mws} 4 \mathrm{mwt}$ \\
\hline $\begin{array}{l}\text { Activin receptor-like kinase-1/ Ki- } \\
\text { nase/ T36959 }\end{array}$ & 2 lcr $3 \mathrm{my} 04$ fao \\
\hline $\begin{array}{l}\text { D-amino acid oxidase/ Oxidoreduc- } \\
\text { tases acting on } \mathrm{CH}-\mathrm{NH} 2 \text { group of } \\
\text { donors/ T33124 }\end{array}$ & $2 \mathrm{du} 82 \mathrm{e} 48$ 2e 49 2e $4 \mathrm{a} 2 \mathrm{e} 82$ 2gnz 3cuk 3g3e 3w4i 3w $4 \mathrm{j} 3 \mathrm{w} 4 \mathrm{k} 3 \mathrm{znn} 3 \mathrm{zno} 3 \mathrm{znp} 3 \mathrm{znq} 4 \mathrm{qfc} 4 \mathrm{qfd}$ \\
\hline Thymidine kinase/ Kinase/ T30081 & $1 \mathrm{w} 4 \mathrm{r} 1 \mathrm{xbt} 2 \mathrm{orv} 2 \mathrm{wvj}$ \\
\hline $\begin{array}{l}\text { Glutathione S-transferase P/ Alkyl } \\
\text { aryl transferase/ T21669 }\end{array}$ & $\begin{array}{l}10 \mathrm{gs} 11 \mathrm{gs} 12 \mathrm{gs} 13 \mathrm{gs} 14 \mathrm{gs} 16 \mathrm{gs} 17 \mathrm{gs} 18 \mathrm{gs} 19 \mathrm{gs} 1 \mathrm{aqv} 1 \mathrm{aqw} 1 \mathrm{aqx} 1 \mathrm{eog} 1 \mathrm{eoh} 1 \mathrm{gss} 1 \mathrm{kbn} 1 \mathrm{lbk} \\
1 \mathrm{md} 31 \mathrm{md} 41 \mathrm{pgt} 1 \mathrm{px} 61 \mathrm{px} 7 \text { } \mathrm{zgn} 20 \mathrm{gs} 22 \mathrm{gs} 2 \mathrm{a} 2 \mathrm{r} 2 \mathrm{a} 2 \mathrm{~s} 2 \mathrm{gss} 2 \mathrm{j} 9 \mathrm{~h} 2 \mathrm{pgt} 3 \mathrm{csh} 3 \mathrm{csi} 3 \mathrm{csj} 3 \mathrm{dd} 3 \\
3 \mathrm{dgq} 3 \mathrm{gss} 3 \mathrm{gus} 3 \mathrm{hjm} 3 \mathrm{hjo} 3 \mathrm{hkr} 3 \mathrm{ie} 3 \mathrm{~km} 63 \mathrm{kmn} 3 \mathrm{kmo} 3 \mathrm{n} 9 \mathrm{j} 3 \mathrm{pgt} 4 \mathrm{gss} 4 \mathrm{pgt} 5 \mathrm{dak} 5 \mathrm{dal} 5 \mathrm{dcg} \\
5 \mathrm{ddl} 5 \mathrm{djl} 5 \mathrm{djm} 5 \mathrm{gss} 5 \mathrm{j} 415 \mathrm{jcw} 5 \mathrm{l} 6 \mathrm{x} 796 \mathrm{gss} 7 \mathrm{gss} 8 \mathrm{gss} 9 \mathrm{gss}\end{array}$ \\
\hline $\begin{array}{l}\text { Isocitrate dehydrogenase (IDH2)/ } \\
\text { Short-chain dehydrogenases reduc- } \\
\text { tases/ T20808 }\end{array}$ & 4 ja8 5 gis 5 i95 5 i96 5svn 5 svo \\
\hline $\begin{array}{l}\text { Phosphodiesterase (PDE) 9/ Phos- } \\
\text { phoric diester hydrolases/ T17852 }\end{array}$ & $\begin{array}{l}\text { 2hd1 2yy2 3dy8 3dyl 3dyn 3dyq 3dys 3jsi 3jsw 3k3e 3k3h 3n3z 3qi3 3qi4 4e90 4g2j 4g2l } \\
4 \mathrm{gh} 64 \mathrm{y} 86 \text { 4y87 4y8c }\end{array}$ \\
\hline $\begin{array}{l}\text { P300/ CBP-associated factor/ } \\
\text { T16902 }\end{array}$ & $\begin{array}{l}\text { 1cm0 } 1 \mathrm{jm} 4 \text { 1n72 } 1 \text { wug } 1 \text { wum } 1 \text { zs5 } 2 \text { rnw } 2 \mathrm{rnx} 3 \mathrm{gg} 3 \text { 4nsq } 5 \mathrm{fdz} 5 \text { fe0 } 5 \text { fe } 15 \mathrm{fe} 25 \mathrm{fe} 35 \mathrm{fe} 45 \mathrm{fe} 5 \\
5 \mathrm{fe} 65 \mathrm{fe} 75 \mathrm{fe} 85 \mathrm{fe} 95 \mathrm{lvq} 5 \mathrm{lvr}\end{array}$ \\
\hline $\begin{array}{l}\text { Lysine specific histone demethy- } \\
\text { lase } 1 / \text { Flavin monoamine oxidase/ } \\
\text { T16739 }\end{array}$ & 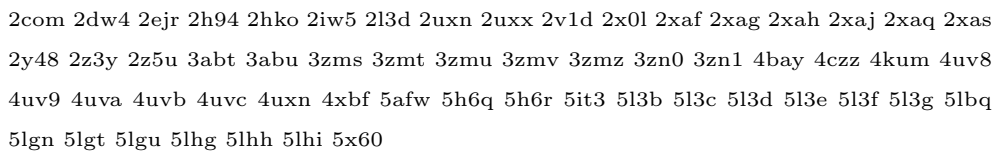 \\
\hline $\begin{array}{l}\text { Protein arginine } \quad \text { N- } \\
\text { methyltransferase } \quad 4 / \quad \text { Methyl- } \\
\text { transferase superfamily/ T12837 }\end{array}$ & $2 \mathrm{y} 1 \mathrm{w} 2 \mathrm{y} 1 \mathrm{x} 4 \mathrm{ikp} 5 \mathrm{dwq} 5 \mathrm{dx} 05 \mathrm{dx} 15 \mathrm{dx} 85 \mathrm{dxa} 5 \mathrm{dxj} 5 \mathrm{u} 4 \mathrm{x}$ \\
\hline mRNA of HER4/ Kinase/ T06559 & 2 ahx 212 t 2 lcx 2 r 4 b 3bbt 3 bbw 3 bce $3 u 2 p ~ 3 u 7 u ~ 3 u 9 u$ \\
\hline
\end{tabular}




\begin{tabular}{|c|c|}
\hline $\begin{array}{l}\text { Trypanothione reductase/ Oxidore- } \\
\text { ductases acting on a sulfur group of } \\
\text { donors/ T99616 }\end{array}$ & $2 \mathrm{wba}$ \\
\hline Integrin alpha-2/ beta-1/ T97353 & 1k11 1lha 3g9w 3t9k 3vi3 3vi4 4dx9 4wjk 4wk0 4wk2 4wk4 \\
\hline $\begin{array}{l}\text { Cytochrome P450 reductase/ Oxi- } \\
\text { doreductases acting on NADH or } \\
\text { NADPH/ T } 95446\end{array}$ & 1b1c 3fjo 3qe2 3qfe 3qfr 3qfs 3qft 5emn 5 fa 6 \\
\hline Integrin alpha-5/ beta-1/ T94306 & $3 \mathrm{vi} 33 \mathrm{vi} 4$ 4wjk $4 \mathrm{wk} 04 \mathrm{wk} 24 \mathrm{wk} 4$ \\
\hline TTR/ T86462 & 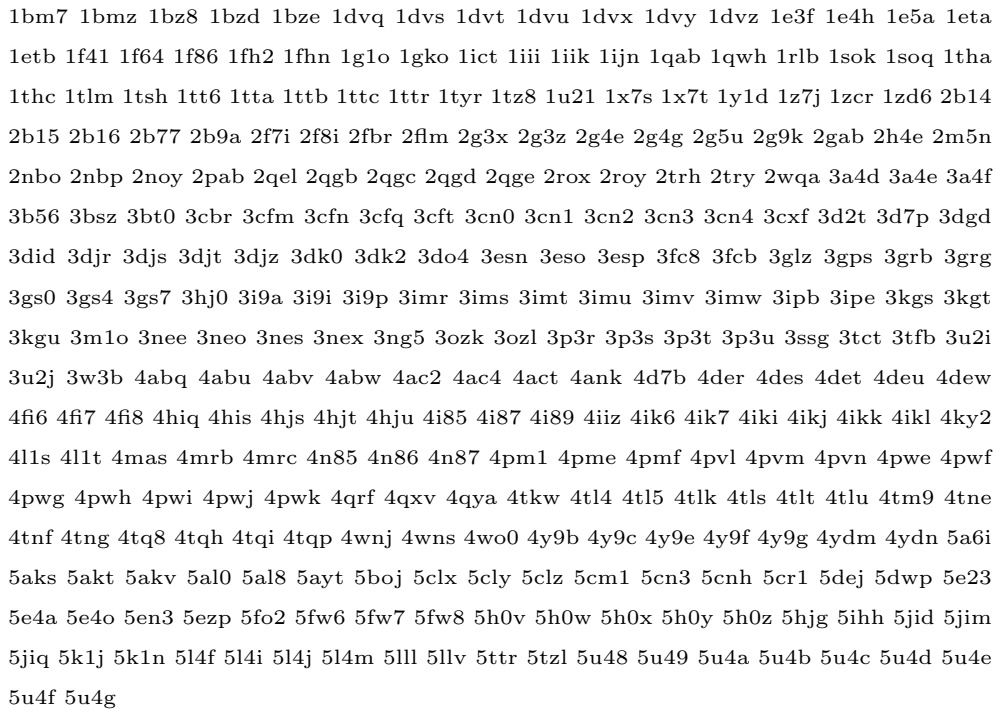 \\
\hline $\begin{array}{l}\text { CDC7 protein kinase/ Kinase/ } \\
\text { T81358 }\end{array}$ & 4 f99 4f9a 4f9b 4f9c 5uwq 5uwr \\
\hline ADAMTS-4/ Peptidase/ T79570 & 2 rjp $3 \mathrm{~b} 2 \mathrm{z} 4 \mathrm{wk} 74$ wke 4 wki \\
\hline $\begin{array}{l}\text { Alpha v beta } 3 \text { integrin receptor/ } \\
\text { Integrin/ T } 72245\end{array}$ & 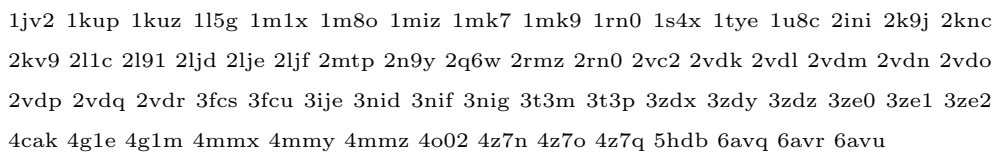 \\
\hline Integrin alpha-5/ beta-3/ T67363 & 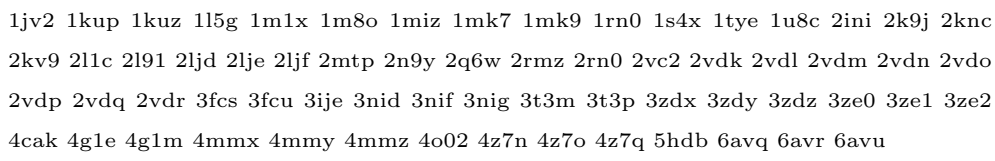 \\
\hline
\end{tabular}




\begin{tabular}{|c|c|}
\hline $\begin{array}{l}\text { Scavenger decapping enzyme DcpS/ } \\
\text { Acid anhydrides hydrolase/ T64074 }\end{array}$ & 1st0 1st4 $1 \mathrm{xml} 1 \mathrm{xmm} 3 \mathrm{bl} 3$ bl9 3bla $4 \mathrm{qde} 4 \mathrm{qdv} 4 \mathrm{qeb}$ \\
\hline $\begin{array}{l}\text { Bone morphogenetic protein recep- } \\
\text { tor/ Kinase/ T60213 }\end{array}$ & $2 \mathrm{hlq} 3 \mathrm{~g} 2 \mathrm{f}$ \\
\hline $\begin{array}{l}\text { Endothelin-converting enzyme } 1 / \\
\text { Peptidase/ T50970 }\end{array}$ & $3 \mathrm{dwb}$ \\
\hline $\begin{array}{l}\text { Nuclear receptor ROR-alpha/ } \mathrm{Nu}- \\
\text { clear hormone receptor/ T43206 }\end{array}$ & $1 \mathrm{n} 831 \mathrm{~s} 0 \mathrm{x} 4 \mathrm{~s} 15$ \\
\hline $\begin{array}{l}\text { 1-deoxy-D-xylulose-5-phosphate re- } \\
\text { ductoisomerase/ Short-chain dehy- } \\
\text { drogenases reductases/ T31751 }\end{array}$ & 3au8 3au9 3aua 3wqq 3wqr 3wqs 4 gae $4 \mathrm{kp} 7$ \\
\hline $\begin{array}{l}\text { Dual specificity mitogen-activated } \\
\text { protein kinase kinase } 5 / \text { Kinase/ } \\
\text { T17143 }\end{array}$ & $2 \mathrm{npt} 2 \mathrm{o} 2 \mathrm{v} 4 \mathrm{ic} 7$ \\
\hline $\begin{array}{l}\text { SET and MYND domain-containing } \\
\text { protein } 2 / \text { Methyltransferase super- } \\
\text { family/ T15797 }\end{array}$ & 3rib 3s7b 3s7d 3s7f 3s7j 3tg4 3tg5 4o6f 4wuy 4ynd 5arf 5arg 5kjk 5kjl 5kjm 5kjn 5wcg \\
\hline Tryptase/ Peptidase/ T14558 & 1lto 2f9n 2f9o 2f9p 2zeb 2zec 4a61 4mpu 4mpv 4mpw 4mpx 4mqa 5 f03 \\
\hline $\begin{array}{l}\text { Valosin-containing protein p97/ } \\
\text { Acid anhydrides hydrolase/ T12646 }\end{array}$ & $\begin{array}{l}\text { 3ebb 3hu1 3hu2 3hu3 3qc8 3qq7 3qq8 3qwz 3tiw 4kdi } 4 \text { kdl } 4 \text { kln } 4 \text { ko8 } 4 \text { kod 4p0a 5b6c 5c18 } \\
\text { 5c19 5c1a 5c1b 5dyg 5dyi 5epp 5ftj } 5 \mathrm{ftk} 5 \mathrm{ftl} 5 \mathrm{ftm} 5 \mathrm{ftn} 5 \mathrm{glf} 5 \text { ifs } 5 \text { ifw } 5 \mathrm{x} 4 \mathrm{l}\end{array}$ \\
\hline $\begin{array}{l}\text { Myosin-7/ TRAFAC class myosin- } \\
\text { kinesin ATPase/ T07507 }\end{array}$ & $\begin{array}{l}\text { 1ik2 } 2 \text { fxm } 2 \text { fxo } 3 \mathrm{dtp} 4 \mathrm{db} 14 \mathrm{p} 7 \mathrm{~h} 4 \mathrm{pa} 04 \mathrm{xa} 14 \mathrm{xa} 34 \mathrm{xa} 44 \mathrm{xa} 65 \mathrm{chx} 5 \mathrm{cj} 05 \mathrm{cj} 15 \mathrm{cj} 45 \text { tby } 5 \mathrm{wj} 7 \\
5 \mathrm{wjb} 5 \text { wlq } 5 \mathrm{wlz} 5 \text { wme }\end{array}$ \\
\hline
\end{tabular}




\begin{tabular}{|c|c|}
\hline $\begin{array}{l}\text { BCAM-AKT2 fusion gene/ Kinase/ } \\
\text { T94621 }\end{array}$ & 1gzk 1gzn 1gzo $1 \mathrm{mrv} 1 \mathrm{mry} 1 \mathrm{o} 6 \mathrm{k} 1 \mathrm{o} 61$ 1p6s 2 jdo 2 jdr 2 uw9 2x39 2xh5 3d0e 3e $873 \mathrm{e} 88$ 3e $8 \mathrm{~d}$ \\
\hline $\begin{array}{l}\text { Thymidine monophosphate kinase/ } \\
\text { Kinase/ T94400 }\end{array}$ & 1g3u 1gsi 1gtv 1mrn 1mrs 1n5i 1n5j 1n5k 1n5l 1w2g 1w2h 4unn 4unp 4unq 4unr 4uns \\
\hline $\begin{array}{l}\text { Corticosteroid-binding globulin/ } \\
\text { Serpin family/ T88452 }\end{array}$ & $2 \mathrm{vdx} 2 \mathrm{vdy} 4 \mathrm{bb} 24 \mathrm{c} 414 \mathrm{c} 49$ \\
\hline $\begin{array}{l}\text { UDP-3-O-[3-hydroxymyristoyl] } \\
\text { n-acetylglucosamine deacety- } \\
\text { lase/ Carbon-nitrogen hydrolase/ } \\
\text { T85944 }\end{array}$ & $\begin{array}{l}\text { 2ves 3p3e 3u1y 3uhm } 4 \text { fw } 34 \text { fw } 4 \text { fw } 54 \text { fw } 64 \text { fw } 74 \text { j3d } 4 \text { lcf } 4 \text { lcg } 4 \text { lch } 4 \text { okg } 5 \text { drq } 5 \text { drr } 5 \mathrm{n} 8 \mathrm{c} \\
5 \text { u39 5u3b 5upg } 5 \mathrm{vwm}\end{array}$ \\
\hline $\begin{array}{l}\text { 3-oxoacyl-[acyl-carrier-protein] syn- } \\
\text { thase II/ Acyltransferase/ T } 82723\end{array}$ & 1b3n 1kas 2gfv 2gfw 2gfx 2gfy $3 g 0 y 3 g 11$ hnz 3 ho2 3 ho9 3i8p \\
\hline $\begin{array}{l}\text { NRH dehydrogenase [quinone] } 2 / \\
\text { Oxidoreductases acting on diphe- } \\
\text { nols as donors/ T75498 }\end{array}$ & $\begin{array}{l}\text { 1qr2 1sg0 1xi2 1zx1 2bzs 2qmy 2qmz 2qr2 2qwx 2qx4 2qx6 2qx8 2qx9 3fw1 3g5m 3gam } \\
\text { 3nfr 3nhf 3nhj 3nhk 3nhl 3nhp 3nhr 3nhs 3nhu 3nhw 3nhy 3o2n 3o73 3ovm 3owh 3owx } \\
\text { 3ox1 3ox2 3ox3 3te7 3tem 3tzb 3uxe 3uxh 4fgj 4fgk 4fgl 4gqi 4gr9 4qod 4qoe 4qof 4qog } \\
\text { 4qoh 4qoi 4qoj 4u7f 4u7g 4u7h 4xdg 4xdh 4zvk 4zvl 4zvm 4zvn 5buc 5lbt 5lbu }\end{array}$ \\
\hline $\begin{array}{l}\text { mRNA of Colony stimulating } \\
\text { factor-1 receptor/ Target of anti- } \\
\text { sense drug/ T55414 }\end{array}$ & 2i0v 2i0y $2 \mathrm{i} 1 \mathrm{~m} 2$ ogv 3 bea $3 \mathrm{dpk} 3 \mathrm{krj} 3 \mathrm{krl} 3$ lcd 3lco $4 \mathrm{dkd} 4 \mathrm{hw} 74$ liq $4 \mathrm{r} 7 \mathrm{~h} 4 \mathrm{r} 7 \mathrm{i} 4 \mathrm{wrl} 4 \mathrm{wrm}$ \\
\hline Plasmepsin 1/ Peptidase/ T50547 & 1lcr 1ldu 3qrv 3qs1 \\
\hline Cdc2-like kinase 1/ Kinase/ T46685 & $1 \mathrm{z} 572 \mathrm{vag} 5 \mathrm{j} 1 \mathrm{v} 5 \mathrm{j} 1 \mathrm{w} 5 \mathrm{x} 8 \mathrm{i}$ \\
\hline $\begin{array}{l}\text { Nicotinate-nucleotide pyrophospho- } \\
\text { rylase [carboxylating]/ Pentosyl- } \\
\text { transferase/ T } 44515\end{array}$ & 1qpn 1qpo 1qpq 1qpr \\
\hline mRNA of HIF2-alpha/ T41003 & 1p97 2a24 3f1n 3f1o 3f1p 3h7w 3h82 4ghi 4gs9 4pky 4xt2 5kiz 5tbm 5ufp \\
\hline
\end{tabular}


Bromodomain-containing protein

4/ Bromodomain/ T40556 2i8n 2lsp 2mjv 2n3k 2ncz 2nd0 2nd1 2nnu 2oss 2ouo 2yel 2yem 3mxf 3p5o 3svf 3svg 3u5j 3u5k 3u5l 3uvw 3uvx 3uvy 3uw9 3zyu 4a9l 4bjx 4bw1 4bw2 4bw3 4bw4 4c66 4c67 4cfk 4cfl 4cl9 4clb 4don 4e96 4f3i 4gpj 4hbv 4hbw 4hbx 4hby 4hxk 4hxl 4hxm 4hxn 4hxo 4hxp 4 hxr 4 hxs 4 ioo 4ioq 4ior 4j0r 4j0s 4j3i 4kv1 4kv4 4lr6 4lrg 4lyi 4lys 4lyw 4lzr 4lzs 4men 4meo 4mep 4meq 4mr3 4mr4 4nqm 4nr8 4nuc 4nud 4nue 4o70 4o71 4o72 4o74 4o75 4o76 4o77 4o78 4o7a 4o7b 4o7c 4o7e 4o7f 4ogi 4ogj 4pce 4pci 4ps5 4qb3 4qr3 4qr4 4qr5 4qzs 4uix 4uiy 4uiz 4uyd 4whw 4wiv 4x2i 4xy9 4xya 4yh3 4yh4 4z1q 4z1s 4z93 4zc9 4zw1 5a5s $5 \mathrm{a} 855 \mathrm{acy} 5 \mathrm{ad} 25 \mathrm{ad} 35 \mathrm{bt} 45 \mathrm{cfw} 5 \mathrm{coi} 5 \mathrm{cp} 55 \mathrm{cpe} 5 \mathrm{cqt} 5 \mathrm{crm} 5 \mathrm{crz} 5 \mathrm{cs} 85 \mathrm{ctl} 5 \mathrm{cy} 95 \mathrm{~d} 0 \mathrm{c} 5 \mathrm{~d} 24$ $5 \mathrm{~d} 255 \mathrm{~d} 265 \mathrm{~d} 3 \mathrm{~h} 5 \mathrm{~d} 3 \mathrm{j} 5 \mathrm{~d} 3 \mathrm{l} 5 \mathrm{~d} 3 \mathrm{n} 5 \mathrm{~d} 3 \mathrm{p} 5 \mathrm{~d} 3 \mathrm{r} 5 \mathrm{~d} 3 \mathrm{~s} 5 \mathrm{~d} 3 \mathrm{t} 5 \mathrm{dlx} 5 \mathrm{dlz} 5 \mathrm{dw} 2$ 5dx4 5e0r 5egu 5ei4 5eis $5 \mathrm{f} 5 \mathrm{z} 5 \mathrm{f} 605 \mathrm{f} 615 \mathrm{f} 625 \mathrm{f} 635 \mathrm{fbx} 5 \mathrm{~h} 21$ 5hcl $5 \mathrm{hls} 5 \mathrm{hm0} 5 \mathrm{hq} 5$ hhq6 5 hq7 5 i 80 5i 88 5igk 5 jwm $5 \mathrm{kdh} 5 \mathrm{khm} 5 \mathrm{kj0} 5 \mathrm{ku} 3 \quad 5 \mathrm{lj} 1 \quad 5 \mathrm{lj} 2 \quad 5$ lrq 5 luu $5 \mathrm{~m} 395 \mathrm{~m} 3 \mathrm{a}$ 5t35 5 ti2 5 ti3 5 ti 4 5ti5 5 ti 6 5 ti7 5u28 5u2c 5u2e 5u2f 5ueo 5uep 5ueq 5uer 5ues 5uet 5ueu 5uev 5uex 5uey 5uez 5ufo 5ula 5uoo 5uvs 5uvt 5uvu 5uvv 5uvw 5uvx 5uvy 5uvz 5vom 5wuu

HIV integrase/ Integrase/ T39087

1a9m 1ajv 1ajx 1axa 1bqm 1bqn 1d4h 1d4i 1d4j 1dlo 1dw6 1ebk 1ebw 1eby 1ebz 1ec0 1 ec1 1ec2 1ec3 1eet 1 g35 1gnm 1gnn 1 gno 1 har 1 hbv 1 hef 1 heg 1 hih $1 \mathrm{hmv} 1 \mathrm{hni} 1 \mathrm{hnv} 1 \mathrm{hos}$ $1 \mathrm{hps} 1 \mathrm{hpz} 1 \mathrm{hqe} 1 \mathrm{hqu} 1 \mathrm{hrh} 1 \mathrm{hte} 1 \mathrm{htf} 1 \mathrm{htg} 1 \mathrm{hvi} 1 \mathrm{hvk} 1 \mathrm{hvp} 1 \mathrm{hvu} 1 \mathrm{hys} 1 \mathrm{ikv} 1 \mathrm{ikw} 1 \mathrm{ikx} 1 \mathrm{iky}$ 1j5o $1 \mathrm{kjh} 1 \mathrm{mer} 1 \mathrm{mes} 1 \mathrm{met} 1 \mathrm{meu} 1 \mathrm{n} 5 \mathrm{y} 1 \mathrm{n} 6 \mathrm{q}$ 1npa $1 \mathrm{npv} 1 \mathrm{npw} 1 \mathrm{qe} 1$ 1qme $1 \mathrm{r} 0 \mathrm{a} 1 \mathrm{rdh} 1 \mathrm{rtd}$ 1rvl 1rvm 1rvn 1rvo 1rvp 1rvq 1rvr 1s6p 1s6q 1s9e 1s9g 1sbg 1suq 1sv5 1t03 1t05 1t7k 1tv6 1tvr 1uwb 1w5v 1w5w 1w5x 1w5y 1yt9 1zp8 1zpa 1zsf 1zsr 2aqu 2b5j 2b6a 2ban 2bbb 2be2 2exf 2g69 2hb3 2hmi 2hnz 2hs1 2hs2 2i4d 2i4u 2i4v 2i4w 2i4x 2i5j 2iaj 2ic3 2idw 2ieo 2jzw 214521462141 2uxz 2uy0 2vg5 2vg6 2vg7 2x4u 2ykm 2ykn 2zd1 2ze2 3avi 3bgr 3dlk 3gga 3ggv 3ggx 3hvt 3ig1 3irx 3is9 3isn 3ith 3jsm 3jyt 3k2p 3k4v 3kle 3klf 3klg 3klh 3kli 3ndt 3nu3 3nu4 3nu5 3nu6 3nu9 3nuj 3nuo 3ok9 3psu 3qaa 3qlh 3qo9 3tkg 3tkw 3t19 3tlh 3v4i 3v6d 3v81 3zps 3zpt 3zpu 4coe 4cp7 4cpq 4cpr 4cps 4cpt 4cpu 4cpw 4cpx 4dg1 4g1q 4g8g 4g8i 4g9d 4g9f 4h4m 4h4o 4i2p 4i2q 4icl 4id5 4idk 4ifv 4ify 4ig0 4ig3 4kfb 4kko 4ko0 4lsl 4lsn 4mfb 4o44 4o4g 4ojr 4pqu 4puo 4pwd 4q0b 4qag 4r5p 4rw4 4rw6 4rw7 4rw8 4rw9 4u8w 4we1 4ye3 4yhq 4zip 4zls 5agz 5ah6 5ah7 5ah8 5ah9 5aha 5ahb 5ahc 5 bry 5bs4 5c24 5c25 5c42 5cym 5cyq 5d3g 5fdl 5hbm 5hlf 5hp1 5hro 5i3u 5i42 5j1e 5jfp 5 jfu 5 jg1 5 t6z 5 t70 5tep 5ter 5tuq 5tw3 5txl 5txm 5txn 5txo 5txp 5ult 5uov 5upz 5uv5 $5 \mathrm{v} 515 \mathrm{v} 5 \mathrm{~m} 5 \mathrm{vqq} 5 \mathrm{vqr} 5 \mathrm{vqs} 5 \mathrm{vqt} 5 \mathrm{vqu} 5 \mathrm{vqv} 5 \mathrm{vqw} 5 \mathrm{vqx} 5 \mathrm{vqy} 5 \mathrm{vqz}$

1ny2 2wok 4asq 4asr 4ecb 4ecc 5i25

Kininogen/ T30731

1a2n 1uae 2z2c 3iss 3kqj 3kr6 3swd

UDP-N-acetylglucosamine

carboxyvinyltransferase/ Alkyl aryl

transferase/ T24634 


\begin{tabular}{|c|c|}
\hline mRNA of transthyretin/ T23389 & 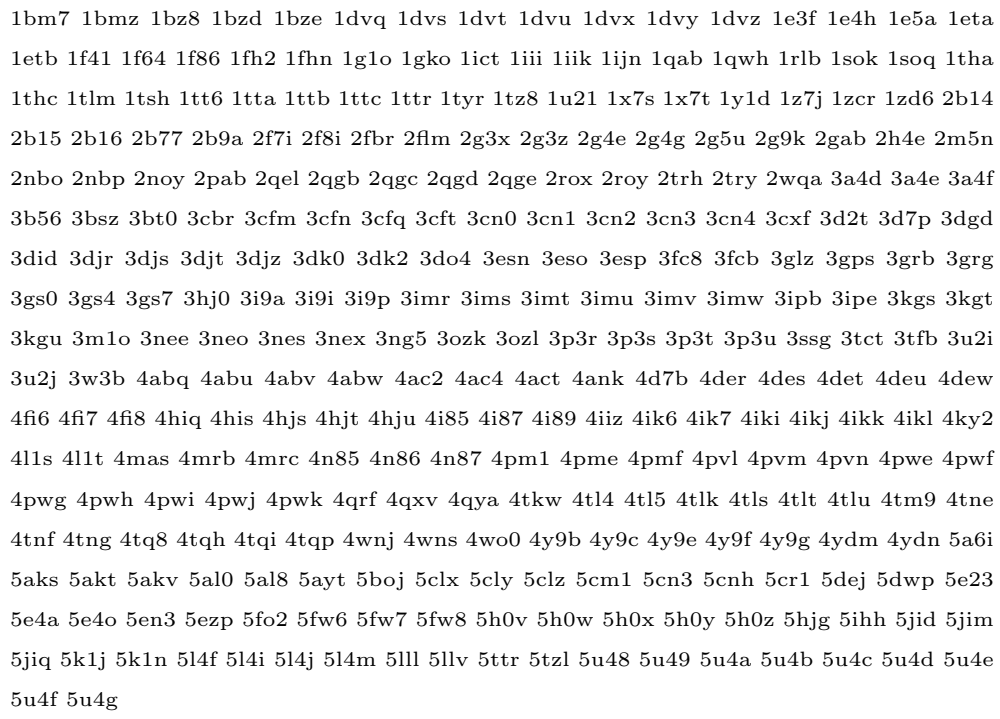 \\
\hline $\begin{array}{l}\text { Pyruvate dehydrogenase kinase iso- } \\
\text { form } 2 \text { (PDHK2)/ Kinase/ T17967 }\end{array}$ & $\begin{array}{l}2 \mathrm{btz} 2 \mathrm{bu} 22 \mathrm{bu} 52 \mathrm{bu} 62 \mathrm{bu} 72 \mathrm{bu} 84 \mathrm{mp} 24 \mathrm{mp} 74 \mathrm{mpc} 4 \mathrm{mpe} 4 \mathrm{mpn} 4 \mathrm{v} 254 \mathrm{v} 265 \mathrm{j} 6 \mathrm{a} 5 \mathrm{j} 715 \mathrm{~m} 4 \mathrm{k} \\
5 \mathrm{~m} 4 \mathrm{~m} 5 \mathrm{~m} 4 \mathrm{n} 5 \mathrm{~m} 4 \mathrm{p}\end{array}$ \\
\hline $\begin{array}{l}\text { Nicotinate-nucleotide adenylyl- } \\
\text { transferase/ Kinase/ T88126 }\end{array}$ & $1 \mathrm{k} 4 \mathrm{k} 1 \mathrm{k} 4 \mathrm{~m}$ \\
\hline $\begin{array}{l}\text { Thioredoxin reductase, cytoplas- } \\
\text { mic/ Oxidoreductases acting on a } \\
\text { sulfur group of donors/ T } 84581\end{array}$ & $1 \mathrm{w} 1 \mathrm{c} 2 \mathrm{cfy} 2 \mathrm{j} 3 \mathrm{n} 2 \mathrm{zzO} 2 \mathrm{zzb} 2 \mathrm{zzc} 3 \mathrm{qfa} 3 \mathrm{qfb}$ \\
\hline $\begin{array}{l}\text { Pyruvate decarboxylase/ Oxidore- } \\
\text { ductases acting on aldehyde or ox- } \\
\text { ogroup of donors/ T71690 }\end{array}$ & 118a 1rp7 2g25 2g28 2g67 2iea 2qta 2qtc \\
\hline $\begin{array}{l}\text { S-nitrosoglutathione reductase/ } \\
\text { Short-chain dehydrogenases reduc- } \\
\text { tases/ T70518 }\end{array}$ & 1wma 2 pfg 3 bhi $3 b h j 3 b h m ~ 4 z 3 d$ \\
\hline $\begin{array}{l}\text { DNA ligase/ Ligases forming phos- } \\
\text { phoric ester bonds/ T70310 }\end{array}$ & 2owo 4 glx 5 tt5 \\
\hline $\begin{array}{l}\text { mRNA of DMPK/ Target of anti- } \\
\text { sense drug/ T } 68766\end{array}$ & $1 \mathrm{wt} 62 \mathrm{vd} 5$ \\
\hline
\end{tabular}




\begin{tabular}{|c|c|}
\hline $\begin{array}{l}\text { Pyruvate kinase M2/ Kinase/ } \\
\text { T65889 }\end{array}$ & $\begin{array}{l}\text { 1t5a 1zjh 3bjf 3bjt 3g2g 3gqy 3gr4 3h6o 3me3 3srd 3srf 3srh 3u2z 4b2d 4fxf 4fxj 4g1n } \\
\text { 4jpg 4qg6 4qg8 4qg9 4qgc 4rpp 4wj8 4yj5 5x1v 5x1w }\end{array}$ \\
\hline $\begin{array}{l}\text { M1-family aminopeptidase/ Pepti- } \\
\text { dase/ T63159 }\end{array}$ & $\begin{array}{l}\text { 3ebg 3ebh 3ebi 3q43 3q44 3t8v 4j3b 4k5l 4k5m 4k5n 4k5o 4k5p 4r5t 4r5v 4r5x 4x2u 4zw3 } \\
4 \mathrm{zw} 5 \text { 4zw6 4zw7 4zw8 4zx3 4zx4 4zx5 4zx6 }\end{array}$ \\
\hline $\begin{array}{l}\text { Tyrosine-protein kinase MELK/ Ki- } \\
\text { nase/ T61518 }\end{array}$ & $\begin{array}{l}\text { 4bky } 4 \text { bkz } 4 \text { bl1 } 4 \mathrm{~d} 2 \mathrm{p} 4 \mathrm{~d} 2 \mathrm{t} 4 \mathrm{~d} 2 \mathrm{v} 4 \mathrm{~d} 2 \mathrm{w} \text { 4ixp } 4 \text { ump } 4 \text { umq } 4 \mathrm{umr} 4 \mathrm{umt} 4 \mathrm{umu} 5 \mathrm{ih} 8 \text { 5ih9 5iha } \\
5 \text { ihc } 5 \mathrm{k} 00\end{array}$ \\
\hline Plasmepsin 2/ Peptidase/ T46465 & $\begin{array}{l}\text { 1j8j 1lee 1lf2 1lf3 1lf4 } 1 \mathrm{~m} 43 \text { 1me6 1pfz 1sme 1w6h 1w6i 1xdh 1xe5 1xe6 2bju 2bl3 2igx } \\
\text { 2igy 2r9b 3f9q 4cku 4y6m 4ya8 4z22 5bwy }\end{array}$ \\
\hline $\begin{array}{l}\text { Proto-oncogene c-RAF/ Kinase/ } \\
\text { T23673 }\end{array}$ & $\begin{array}{l}\text { 1c1y 1faq 1far 1gua 1rfa 3cu8 3iqj 3iqu 3iqv 3kuc 3kud 3nkx 3o8i 3omv 4fj3 4g0n 4g3x } \\
\text { 4iea 4ihl }\end{array}$ \\
\hline Gelsolin/ T23000 & 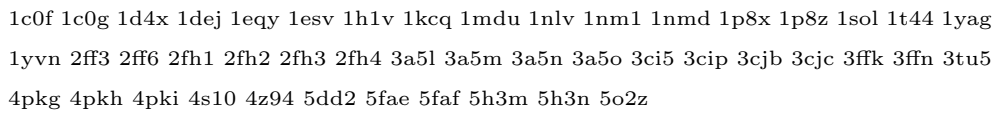 \\
\hline $\begin{array}{l}\text { Deoxyribonucleoside phosphotrans- } \\
\text { ferase/ Kinase/ T22976 }\end{array}$ & 1j90 1oe0 1ot3 $1 \mathrm{zm} 7$ 1zmx $2 \mathrm{jcs} 2 \mathrm{jj} 82 \mathrm{vp} 02 \mathrm{vp} 2$ 2vp4 2vp5 2vp6 2vp9 2vpp 2vqs \\
\hline $\begin{array}{l}\text { Glycosyltransferase MurG/ Hexo- } \\
\text { syltransferase/ T07466 }\end{array}$ & $1 \mathrm{fOk} 1 \mathrm{nlm}$ \\
\hline $\begin{array}{l}\text { BMX non-receptor tyrosine kinase/ } \\
\text { T99990 }\end{array}$ & 2 ekx 2ys2 3sxr 3sxs \\
\hline casein kinase 1, epsilon/ T99989 & $4 \mathrm{hni} 4 \mathrm{hok}$ \\
\hline $\begin{array}{l}\text { receptor (TNFRSF)-interacting } \\
\text { serine-threonine kinase 1/ T99340 }\end{array}$ & 4 ith 4 iti 4 itj 4 neu 5 hx 65 tx 5 \\
\hline $\begin{array}{l}\text { [Pyruvate dehydrogenase } \\
\text { [lipoamide]] kinase isozyme } 3, \\
\text { mitochondrial/ Kinase/ T99029 }\end{array}$ & $1 \mathrm{y} 8 \mathrm{n} 1 \mathrm{y} 8 \mathrm{o} 1 \mathrm{y} 8 \mathrm{p} 2 \mathrm{pnr} 2 \mathrm{q} 8 \mathrm{i}$ \\
\hline histone deacetylase $7 /$ T98698 & $3 \mathrm{c} 0 \mathrm{y} 3 \mathrm{c} 0 \mathrm{z} 3 \mathrm{c} 103 \mathrm{znr} 3 \mathrm{zns}$ \\
\hline $\begin{array}{l}\text { Thymidylate synthase/ Methyl- } \\
\text { transferase superfamily/ T98397 }\end{array}$ & $\begin{array}{l}\text { 1hvy } 1 \text { hw } 3 \text { hw } 4 \text { haw } 1 \text { i00 1ju6 1juj 1ypv 2onb 2rd8 2rda 3eaw 3ebu 3ed7 3edw 3ef9 3egy } \\
\text { 3ehi 3ej1 3gg5 3gh0 3gh2 3h9k 3hb8 3n5e 3n5g 3ob7 4e28 4fgt 4g2o 4g6w 4gd7 4gyh 4h1i } \\
4 \text { jef 4kpw 4o1u 4o1x 4up1 5hs3 5wrn 5x4w 5x4x 5x4y 5x5a 5x5d 5x5q 5x66 5x67 5x69 }\end{array}$ \\
\hline $\begin{array}{l}\text { Peptidyl-prolyl cis-trans isomerase } \\
\text { FKBP5/ Cis-trans-isomerases/ } \\
\text { T98337 }\end{array}$ & $\begin{array}{l}\text { 1kt0 3o5d 3o5e 3o5f 3o5g 3o5i 3o5j 3o5k 3o5l 3o5m 3o5o 3o5p 3o5q 3o5r 4drh 4dri 4drk } \\
4 \text { drm } 4 \text { drn } 4 \text { dro } 4 \text { drp } 4 \text { drq } 4 \text { jfi } 4 \text { jfj } 4 \text { jfk } 4 \text { jfl } 4 \text { jfm 4r0x } 4 \text { tw6 } 4 \text { tw } 74 \text { tx0 4w9o } 4 \text { w9p 4w9q } \\
5 \text { bxj } 5 \text { dit } 5 \text { diu } 5 \text { div } 5 \text { njx } 5 \text { omp }\end{array}$ \\
\hline
\end{tabular}




\begin{tabular}{|c|c|}
\hline $\begin{array}{l}\text { Deoxycytidine kinase/ Kinase/ } \\
\text { T98089 }\end{array}$ & $\begin{array}{l}\text { 1p5z 1p60 1p61 1p62 2a2z 2a30 2a7q 2no0 2no1 2no6 2no7 2no9 2noa 2qrn 2qro 2zi3 2zi4 } \\
\text { 2zi5 2zi6 2zi7 2zi9 2zia 3hp1 3ipx 3ipy 3kfx 3mjr 3qej 3qen 3qeo 4jlj 4jlk 4jlm 4jln 4kcg } \\
\text { 4l5b 4q18 4q19 4q1a 4q1b 4q1c 4q1d 4q1e 4q1f }\end{array}$ \\
\hline ADAM 8/ Peptidase/ T95761 & $4 \mathrm{dd} 8$ \\
\hline $\begin{array}{l}\text { mitogen-activated protein kinase } \\
\text { kinase kinase } 14 / \text { T } 95736\end{array}$ & $4 \mathrm{dn} 54 \mathrm{~g} 3 \mathrm{~d} 4 \mathrm{idt} 4 \mathrm{idv}$ \\
\hline $\begin{array}{l}\text { mRNA of MMP9/ Peptidase/ } \\
\text { T93690 }\end{array}$ & 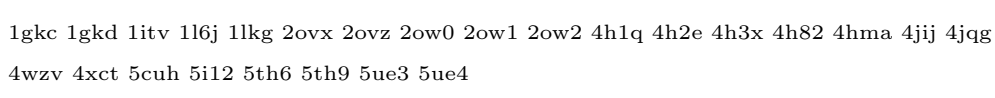 \\
\hline $\begin{array}{l}\text { Prostatic acid phosphatase/ } \\
\text { T93283 }\end{array}$ & 1cvi 1nd5 1nd6 2hpa 213h $217721792 \mathrm{mg} 03 \mathrm{ppd}$ \\
\hline $\begin{array}{lr}\text { dual-specificity } & \text { tyrosine- }(\mathrm{Y})- \\
\text { phosphorylation } & \text { regulated kinase } \\
\text { 1A/ T92803 } & \end{array}$ & $\begin{array}{l}\text { 2vx3 2wo6 3anq 3anr 4aze 4mq1 4mq2 4nct 4ylj 4ylk 4yll 4yu2 5a3x 5a4e 5a4l 5a4q 5a4t } \\
\text { 5a54 5aik }\end{array}$ \\
\hline $\begin{array}{l}\text { Replicase polyprotein 1ab/ Viral } \\
\text { RNA helicase/ T92724 }\end{array}$ & $\begin{array}{l}\text { 1o5s 1p76 1p9t 1pa5 1puk 1q1x 1q2w 1qz8 1sxf 1uj1 1uk2 1uk3 1uk4 1uw7 1wof 1ysy 1z1i } \\
\text { 1z1j 2a5a 2a5i 2a5k 2acf 2ahm 2aj5 2alv 2amd 2amq 2bx3 2bx4 2c3s 2d2d 2duc 2fav 2fe8 } \\
\text { 2fyg 2g1f 2g9t 2ga6 2gdt 2gri 2gt7 2gt8 2gtb 2gx4 2gz7 2gz8 2gz9 2h2z 2h85 2hob 2hsx } \\
\text { 2idy 2jzd 2jze 2jzf 2k87 2op9 2ozk 2pwx 2q6g 2qc2 2qcy 2qiq 2rhb 2rnk 2v6n 2vj1 2xyq } \\
\text { 2xyr 2xyv 2z3c 2z3d 2z3e 2z94 2z9g 2z9j 2z9k 2z9l 3d62 3e9s 3ebn 3r24 4tww 4twy 4wy3 } \\
\text { 4zuh 5b6o 5c5n 5c5o 5c8s 5c8t 5c8u 5e6j 5f22 }\end{array}$ \\
\hline $\begin{array}{l}\text { Pantothenate kinase/ Kinase/ } \\
\text { T91895 }\end{array}$ & 1 esm 1 esn 1 sq 5 \\
\hline ADAMTS1/ T90987 & 2 jih $2 \mathrm{v} 4 \mathrm{~b} 3 \mathrm{q} 2 \mathrm{~g} 3 \mathrm{q} 2 \mathrm{~h}$ \\
\hline $\begin{array}{l}\text { Adenylosuccinate synthetase/ } \\
\text { Carbon-nitrogen ligase/ T89054 }\end{array}$ & $1 \mathrm{p} 9 \mathrm{~b}$ \\
\hline Integrin alpha-V/ beta-1/ T88806 & 1k11 1lha 3g9w 3t9k 3vi3 3vi4 4dx9 4wjk 4wk0 4wk2 4wk4 \\
\hline $\begin{array}{l}\text { L-lactate dehydrogenase/ Short- } \\
\text { chain dehydrogenases reductases/ } \\
\text { T88057 }\end{array}$ & 1sov 1 sow $3 \mathrm{czm}$ \\
\hline casein kinase 1 , delta/ T87109 & $\begin{array}{l}\text { 3uys 3uyt 3uzp } 4 \text { hgt } 4 \mathrm{hnf} 4 \mathrm{~kb} 84 \mathrm{kba} 4 \mathrm{kbc} 4 \mathrm{kbk} 4 \text { tn6 } 4 \text { tw9 } 4 \text { twc } 5 \text { ih } 4 \text { 5ih5 5ih6 5mqv } \\
5 \mathrm{w} 4 \mathrm{w}\end{array}$ \\
\hline $\begin{array}{l}\text { Scytalone dehydratase/ Carbon- } \\
\text { oxygen lyases/ T86829 }\end{array}$ & 1idp 1std 2std 3std 4std 5std 6std 7std \\
\hline
\end{tabular}




\begin{tabular}{|c|c|}
\hline $\begin{array}{l}\mathrm{NH}(3) \text {-dependentNAD }(+) \text { syn- } \\
\text { thetase/ Carbon-nitrogen ligase/ } \\
\text { T86152 }\end{array}$ & $1 \mathrm{wxe} 1 \mathrm{wxf} 1 \mathrm{wxg} 1 \mathrm{wxh} 1 \mathrm{wxi}$ \\
\hline mRNA of cytohesin-2/ T85056 & $1 \mathrm{pbv} 1 \mathrm{r} 8 \mathrm{~m} 1 \mathrm{r} 8 \mathrm{q} 1 \mathrm{r} 8 \mathrm{~s} 1 \mathrm{~s} 9 \mathrm{~d} 4 \mathrm{jmi} 4 \mathrm{jmo} 4 \mathrm{jwl} 4 \mathrm{jxh} 415 \mathrm{~m} 4 \mathrm{z} 21$ \\
\hline $\begin{array}{l}\text { Prostacyclin synthase/ Intramolec- } \\
\text { ular oxidoreductases/ T } 83979\end{array}$ & 2 iag $3 \mathrm{~b} 6 \mathrm{~h}$ \\
\hline Quinone oxidoreductase/ T83284 & $1 \mathrm{yb} 5$ \\
\hline $\begin{array}{l}\text { ribosomal protein } \mathrm{S} 6 \text { kinase, } 90 \mathrm{kDa} \text {, } \\
\text { polypeptide } 1 / \mathrm{T} 83202\end{array}$ & 2wnt 2z7q 2z7r 2z7s 3rny 3tei 4h3p 4nif 5csf 5 csi $5 \operatorname{csj} 5 \operatorname{csn} 5$ n7d 5 nff 5 n7g 5 v61 5v62 \\
\hline $\begin{array}{l}\text { ABL proto-oncogene } 2, \quad \text { non- } \\
\text { receptor tyrosine kinase/ } \mathrm{T} 83009\end{array}$ & 2 ecd $2 \mathrm{kk} 12 \mathrm{xyn} 3 \mathrm{gvu} 3 \mathrm{hmi} 3$ ulr 4 eih \\
\hline $\begin{array}{l}\text { HCV helicase/ Acid anhydrides hy- } \\
\text { drolase/ T } 82916\end{array}$ & 1rtl 2a4g 2gvf 3eyd 3hkw 3kn2 3qgh 3qgi 3rc4 3rc5 3su4 \\
\hline casein kinase 1 , gamma $2 / \mathrm{T} 82773$ & $2 \mathrm{c} 47$ \\
\hline $\begin{array}{l}\text { Kynurenine oxoglutarate transami- } \\
\text { nase II/ Transferases of nitrogenous } \\
\text { groups/ T } 82668\end{array}$ & 2qlr 2r2n 2vgz 2xh1 3dc1 3ue8 4gdy 4ge4 4ge7 4ge9 4geb 5efs 5eun 5tf5 \\
\hline $\begin{array}{l}\text { Folate receptor beta/ Folate recep- } \\
\text { tor/ T } 82297\end{array}$ & $4 \mathrm{kmy} 4 \mathrm{kmz} 4 \mathrm{kn} 04 \mathrm{kn} 14 \mathrm{kn} 2$ \\
\hline $\begin{array}{l}\text { Calmodulin-dependent kinase II/ } \\
\text { T82262 }\end{array}$ & 2zv2 5uy6 5uyj 5vt1 \\
\hline $\begin{array}{l}\text { death-associated protein kinase } 3 / \\
\text { T81623 }\end{array}$ & 1yrp 2j90 3bhy 3bqr 5a6n 5a6o \\
\hline $\begin{array}{l}\text { DNA gyrase B subunit/ ATP- } \\
\text { hydrolyzing DNA topoisomerase/ } \\
\text { T81503 }\end{array}$ & $\begin{array}{l}\text { 1aj6 1ei1 1kzn 3g7e 3nuh 4duh 4hyp 4kfg 4prv 4prx 4pu9 4wub 4wuc 4wud 4xtj 4zvi 513j } \\
5 \mathrm{mmn} 5 \mathrm{mmo} 5 \mathrm{mmp}\end{array}$ \\
\hline $\begin{array}{l}\text { casein kinase } 2, \text { alpha prime } \\
\text { polypeptide subunit/ } \mathrm{T} 80526\end{array}$ & 3e3b 3 ofm $3 \mathrm{u} 875 \mathrm{~m} 4 \mathrm{u} 5 \mathrm{~m} 56$ \\
\hline $\begin{array}{l}\text { Von Hippel-Lindau disease tumor } \\
\text { suppressor (VHL)/ T80423 }\end{array}$ & $\begin{array}{l}\text { 1lm8 1lqb 1vcb 3zrc 3zrf 3ztc 3ztd 3zun 4ajy 4awj 4b95 4b9k 4bks 4bkt 4w9c 4w9d 4w9e } \\
4 \text { w9f 4w9g 4w9h 4w9i 4w9j 4w9k 4w9l 4wqo 5lli 5n4w 5nvv 5nvw 5nvx 5nvy 5nvz 5nw0 } \\
\text { 5nw1 5nw2 5t35 }\end{array}$ \\
\hline
\end{tabular}




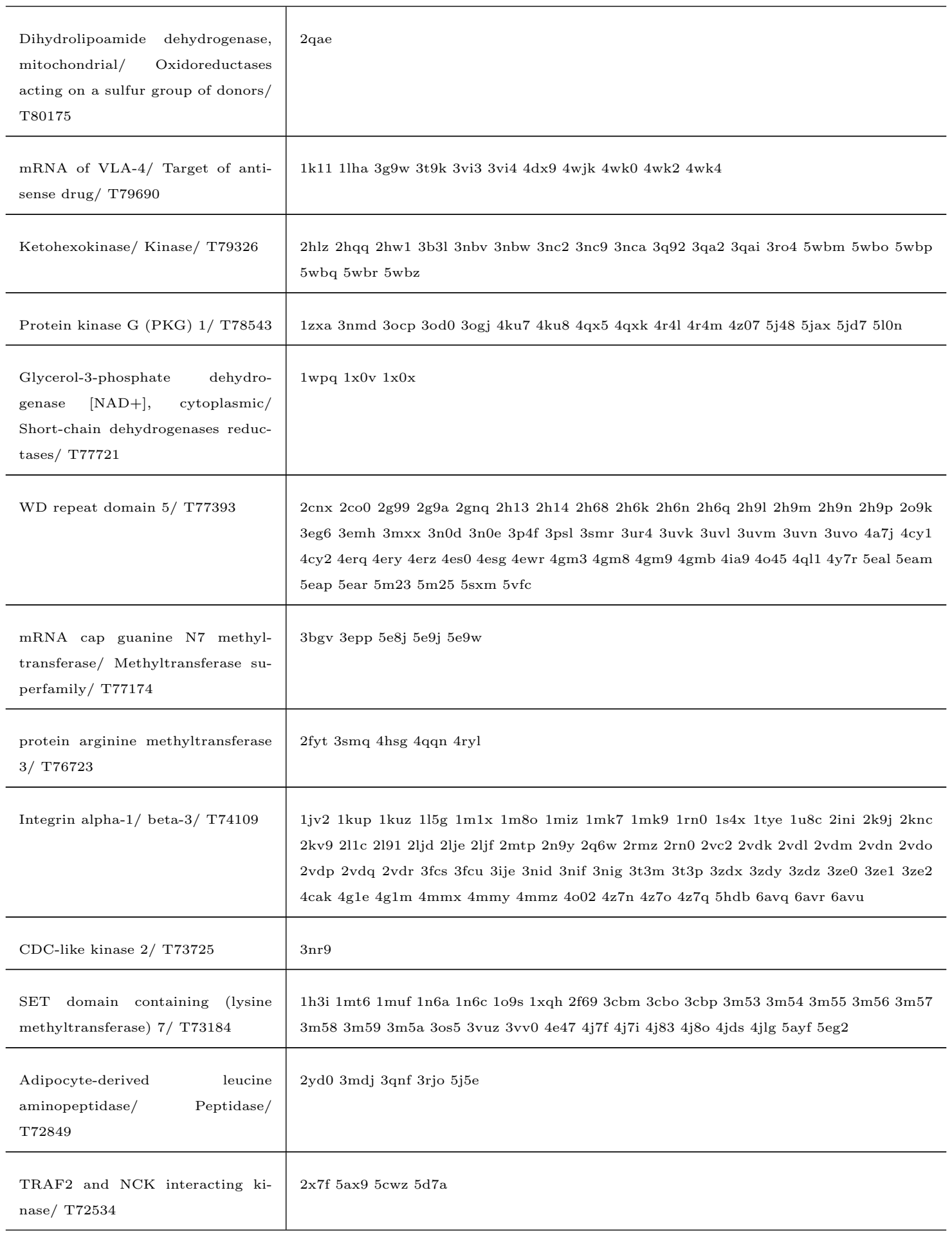




\begin{tabular}{|c|c|}
\hline Caspase $7 / \mathrm{T} 72252$ & 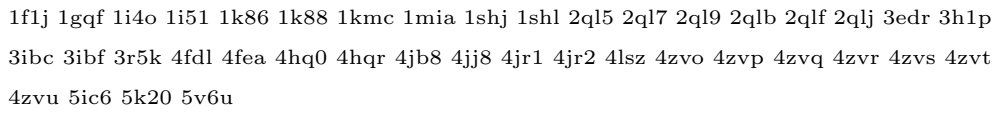 \\
\hline GAR transformylase/ T72042 & 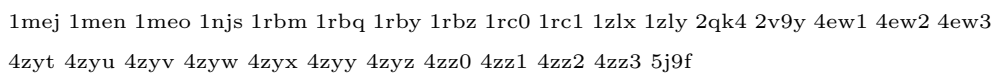 \\
\hline $\begin{array}{l}\text { Neutrophil gelatinase-associated } \\
\text { lipocalin/ Calycin family/ T72010 }\end{array}$ & 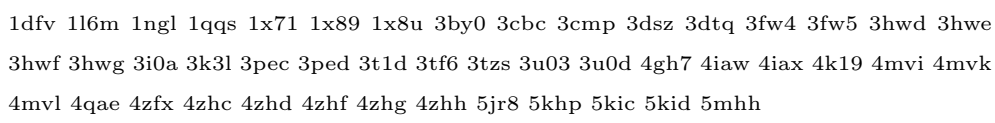 \\
\hline GTPase HRas/ Ras family/ T71081 & 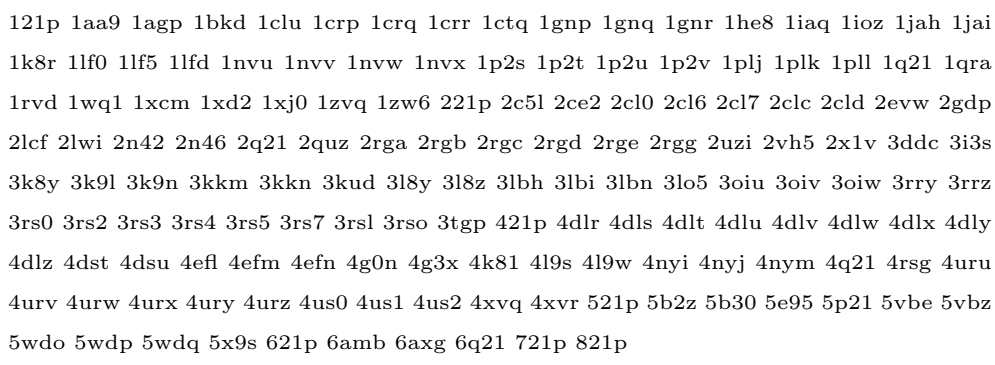 \\
\hline $\begin{array}{l}\text { mitogen-activated protein kinase } 7 / \\
\text { T70851 }\end{array}$ & 2q8y 4 b99 4 ic7 4 ic 8 4zsg 4 zsj 4 zsl 5 byy 5 byz \\
\hline $\begin{array}{l}\text { mRNA of human zinc finger } \\
\text { protein-217/ Zinc-finger/ T70278 }\end{array}$ & $2 \mathrm{hu} 23 \mathrm{uk} 3 \mathrm{ff} 2 \mathrm{j} 4 \mathrm{is} 1$ \\
\hline $\begin{array}{l}\text { Nuclear receptor binding SET do- } \\
\text { main containing protein } 1 / \text { Methyl- } \\
\text { transferase superfamily/ T } 68777\end{array}$ & 3ooi \\
\hline $\begin{array}{l}\text { Poly(ADP-ribose) glycohydrolase/ } \\
\text { Glycosylases/ T66935 }\end{array}$ & $4 \mathrm{a} 0 \mathrm{~d} 4 \mathrm{~b} 1 \mathrm{~g}$ 4b1h 4b1i 4b1j 5a7r 5lhb \\
\hline $\begin{array}{l}\text { Endoplasmic reticulum aminopepti- } \\
\text { dase } 2 / \mathrm{T} 65818\end{array}$ & $3 \mathrm{se} 64 \mathrm{e} 364 \mathrm{jbs} 5 \mathrm{ab0} 5 \mathrm{ab} 25 \mathrm{cu} 55 \mathrm{j} 6 \mathrm{~s} 5 \mathrm{k} 1 \mathrm{v}$ \\
\hline $\mathrm{HCN} 2 / \mathrm{T} 65005$ & $2 \mathrm{mpf} 3 \mathrm{u} 10$ \\
\hline $\begin{array}{l}\text { Angiotensinogen/ Serpin family/ } \\
\text { T64485 }\end{array}$ & 1n9u 1n9v 2jp8 2wxw 2x0b 3ck0 3woo 3wor 4aa1 4aph 4fys 5e2q \\
\hline $\begin{array}{l}\text { cyclin-dependent kinase-like } 1 \\
(\mathrm{CDC} 2 \text {-related kinase)/ T63851 }\end{array}$ & 4agu \\
\hline
\end{tabular}




\begin{tabular}{|c|c|}
\hline $\begin{array}{l}\text { UDP-N- } \\
\text { acetylenolpyruvylglucosamine } \\
\text { reductase/ Short-chain dehydroge- } \\
\text { nases reductases/ T63359 }\end{array}$ & $1 \mathrm{mbb} 1 \mathrm{mbt} 1 \mathrm{uxy} 2 \mathrm{mbr} 2 \mathrm{q} 85$ \\
\hline Retinoid X receptor-beta/ T60077 & 1h9u 1 uhl 5 hjp $5 \mathrm{i} 4 \mathrm{v} 5 \mathrm{kya} 5 \mathrm{kyj}$ \\
\hline $\begin{array}{l}\text { Estrogen-related receptor-gamma/ } \\
\text { T59875 }\end{array}$ & $1 \mathrm{kv} 61 \mathrm{tfc} 1 \mathrm{vjb} 2 \mathrm{e} 2 \mathrm{r} 2 \mathrm{ewp} 2 \mathrm{gp} 72 \mathrm{gpo} 2 \mathrm{gpp} 2 \mathrm{gpu} 2 \mathrm{gpv} 2 \mathrm{p} 7 \mathrm{a} 2 \mathrm{p} 7 \mathrm{~g} 2 \mathrm{p} 7 \mathrm{z} 2 \mathrm{zas} 2 \mathrm{zbs} 2 \mathrm{zkc}$ \\
\hline $\begin{array}{l}\text { mRNA of Protein kinase } \mathrm{C} \text { alpha/ } \\
\text { Kinase/ T56722 }\end{array}$ & 2eli 3iw4 4dnl 4ra4 \\
\hline $\begin{array}{l}\text { Phenylethanolamine } \\
\text { methyltransferase/ T56496 }\end{array}$ & $\begin{array}{l}\text { 1hnn } 1 \mathrm{n} 7 \mathrm{i} 1 \mathrm{n} 7 \mathrm{j} 1 \mathrm{yz} 32 \mathrm{an} 32 \mathrm{an} 42 \mathrm{an} 52 \mathrm{~g} 702 \mathrm{~g} 712 \mathrm{~g} 72 \text { 2g8n } 2 \mathrm{obf} 2 \mathrm{ony} 2 \mathrm{onz} 2 \mathrm{opb} 3 \mathrm{hca} 3 \mathrm{hcb} \\
3 \mathrm{hcc} 3 \mathrm{hcd} 3 \mathrm{hce} 3 \mathrm{hcf} 3 \mathrm{kpj} 3 \mathrm{kpu} 3 \mathrm{kpv} 3 \mathrm{kpw} 3 \mathrm{kpy} 3 \mathrm{kqm} 3 \mathrm{kqo} 3 \mathrm{kqp} 3 \mathrm{kqq} 3 \mathrm{kqs} 3 \mathrm{kqt} 3 \mathrm{kqv} \\
\text { 3kqw } 3 \mathrm{kqy} 3 \mathrm{kr} 03 \mathrm{kr} 13 \mathrm{kr} 24 \mathrm{dm} 34 \mathrm{mik} 4 \mathrm{mq} 4\end{array}$ \\
\hline $\begin{array}{l}\text { lysine (K)-specific demethylase } 6 \mathrm{~B} / \\
\text { T56057 }\end{array}$ & $2 \mathrm{xue} 2 \mathrm{xxz} 4$ ask $5 \mathrm{fp} 35$ oy 3 \\
\hline $\begin{array}{l}\text { Schistosoma mansoni purine nucle- } \\
\text { oside phosphorylase/ T54519 }\end{array}$ & 1tcu 1 tcv 1 td 13 djf $3 \mathrm{e} 0 \mathrm{q} 3 \mathrm{e} 9 \mathrm{r} 3 \mathrm{e} 9 \mathrm{z} 3 \mathrm{f} 8 \mathrm{w} 3 \mathrm{faz} 3 \mathrm{fb} 13 \mathrm{fnq} 3 \mathrm{iex}$ \\
\hline $\begin{array}{l}\text { Cyclin-dependent protein kinase } \\
\text { PfPK5/ Kinase/ T54472 }\end{array}$ & 1lch $1 \mathrm{ob} 31 \mathrm{v} 0 \mathrm{~b} 1 \mathrm{v} 0 \mathrm{o} 1 \mathrm{v} 0 \mathrm{p}$ \\
\hline $\begin{array}{l}\text { euchromatic histone-lysine } \quad \mathrm{N}- \\
\text { methyltransferase } 2 / \mathrm{T} 53251\end{array}$ & $2 \mathrm{o} 8 \mathrm{j} 3 \mathrm{dm} 13 \mathrm{k} 5 \mathrm{k} 3 \mathrm{rjw} 4 \mathrm{nvq} 5 \mathrm{jhn} 5 \mathrm{jin} 5 \mathrm{jiy} 5 \mathrm{jj} 05 \mathrm{t} 0 \mathrm{k} 5 \mathrm{t} 0 \mathrm{~m} 5$ ttf 5 tuy $5 \mathrm{vsc} 5 \mathrm{vse}$ \\
\hline $\begin{array}{l}\text { Acidic mammalian chitinase/ Gly- } \\
\text { cosylases/ T51597 }\end{array}$ & 2 ybt 2 ybu 3 fxy 3 fy $13 \mathrm{rm} 4$ 3rm8 3rm9 3rme \\
\hline $\begin{array}{l}\text { Tryptophan hydroxylase/ Oxidore- } \\
\text { ductases acting on paired donors/ } \\
\text { T51593 }\end{array}$ & $1 \mathrm{in} 91 \mathrm{mlw} 3 \mathrm{hf} 63 \mathrm{hf} 83 \mathrm{hfb} 5 \mathrm{j} 6 \mathrm{~d} 51015 \mathrm{tpg}$ \\
\hline
\end{tabular}




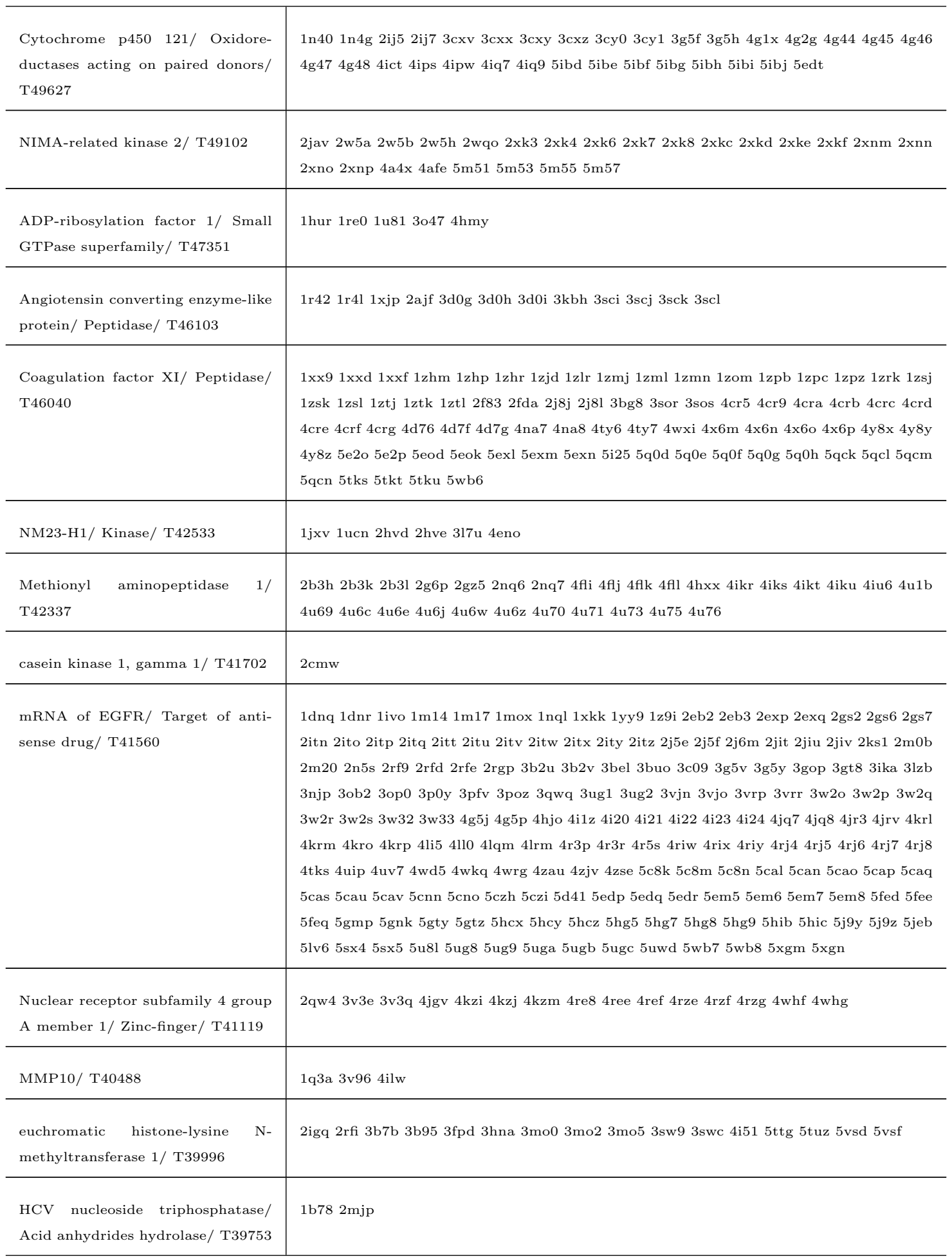




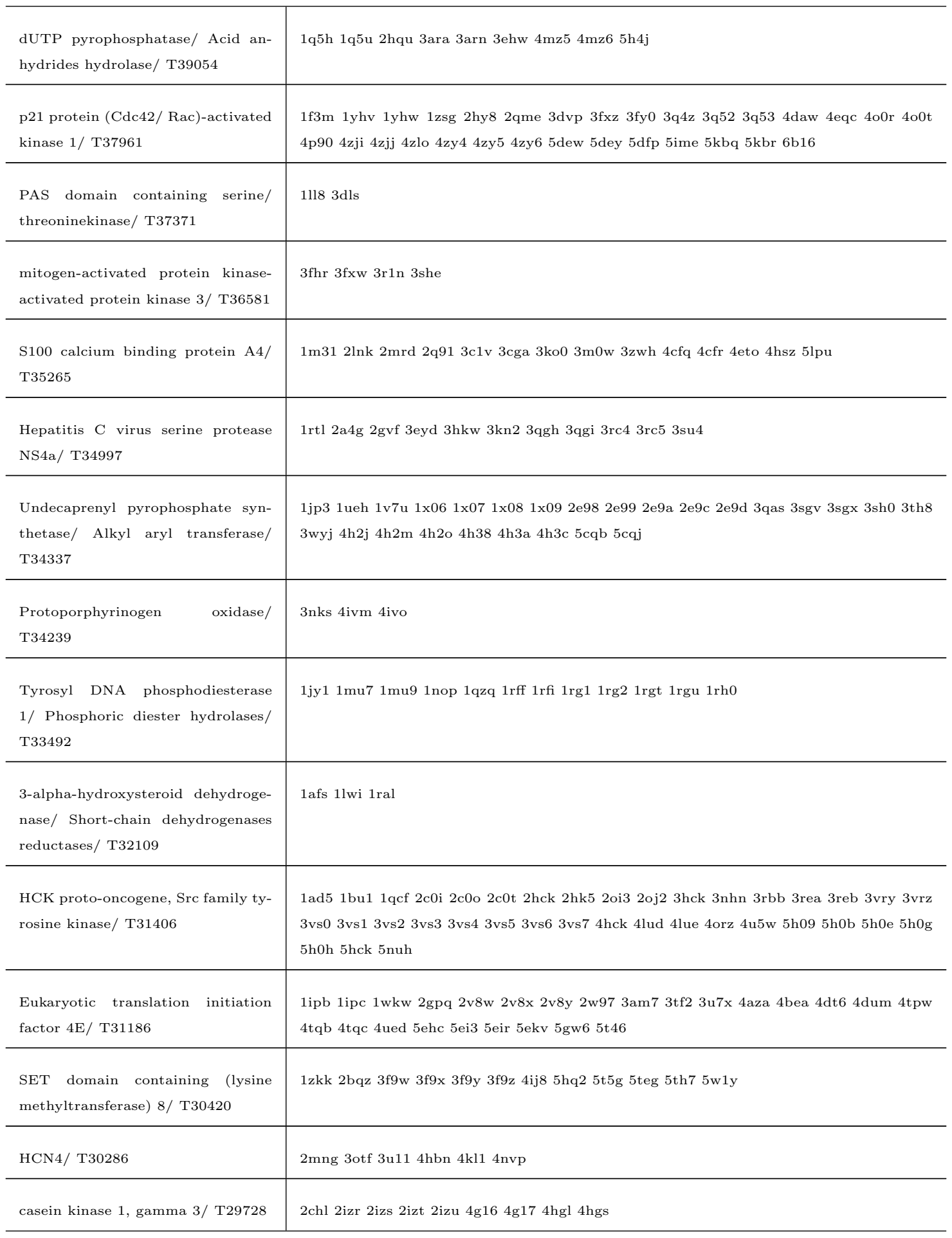




\begin{tabular}{|c|c|}
\hline $\begin{array}{l}\text { Ketol-acid reductoisomerase/ } \\
\text { Short-chain dehydrogenases reduc- } \\
\text { tases/ T28153 }\end{array}$ & $1 \mathrm{yrl} 3 \mathrm{ulk}$ \\
\hline Integrin beta-3/ Integrin/ T26457 & 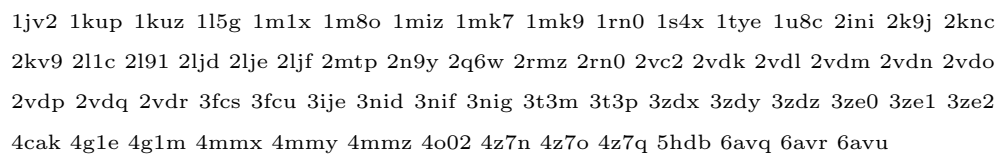 \\
\hline Fibrinogen/ Fibrinogen/ T26144 & 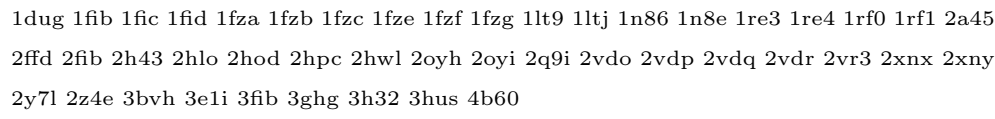 \\
\hline $\begin{array}{l}\text { Mixed-lineage leukemia protein } \\
\text { (MLL) complex/ Methyltransferase } \\
\text { superfamily/ T25462 }\end{array}$ & $\begin{array}{l}\text { 2agh 2j2s 2jyi } 2 \mathrm{kkf} 2 \mathrm{ku} 72 \mathrm{kyu} 2 \text { lxs } 2 \text { lxt } 2 \mathrm{msr} 2 \mathrm{mtn} 2 \mathrm{w} 5 \mathrm{y} 2 \mathrm{w} 5 \mathrm{z} 3 \mathrm{eg} 63 \mathrm{emh} 3 \text { lqh 3lqi 3lqj } \\
3 \mathrm{p} 4 \mathrm{f} 3 \mathrm{u} 853 \mathrm{u} 884 \mathrm{esg} 4 \mathrm{gq} 64 \mathrm{nw} 35 \mathrm{f} 5 \mathrm{e} 5 \mathrm{f} 61\end{array}$ \\
\hline $\begin{array}{l}\text { RAR-related orphan receptor- } \\
\text { gamma/ T25307 }\end{array}$ & 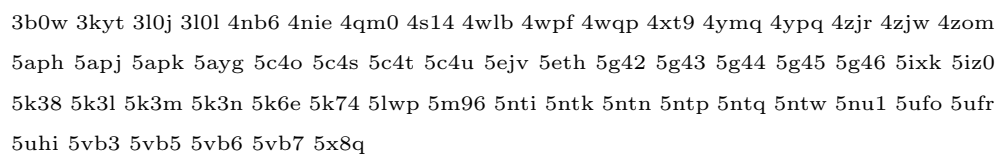 \\
\hline $\begin{array}{l}\text { 3-Deoxy-D-manno-octulosonate } 8 \text { - } \\
\text { phosphate synthase/ Alkyl aryl } \\
\text { transferase/ T23688 }\end{array}$ & $1 \mathrm{~d} 9 \mathrm{e} 1 \mathrm{~g} 7 \mathrm{u} 1 \mathrm{~g} 7 \mathrm{v} 1 \mathrm{gg} 01 \mathrm{phq} 1 \mathrm{phw} 1 \mathrm{pl} 9$ 1q3n $1 \mathrm{x} 6 \mathrm{u} 1 \mathrm{x} 8 \mathrm{f}$ \\
\hline HIV p24 capsid protein/ T23343 & 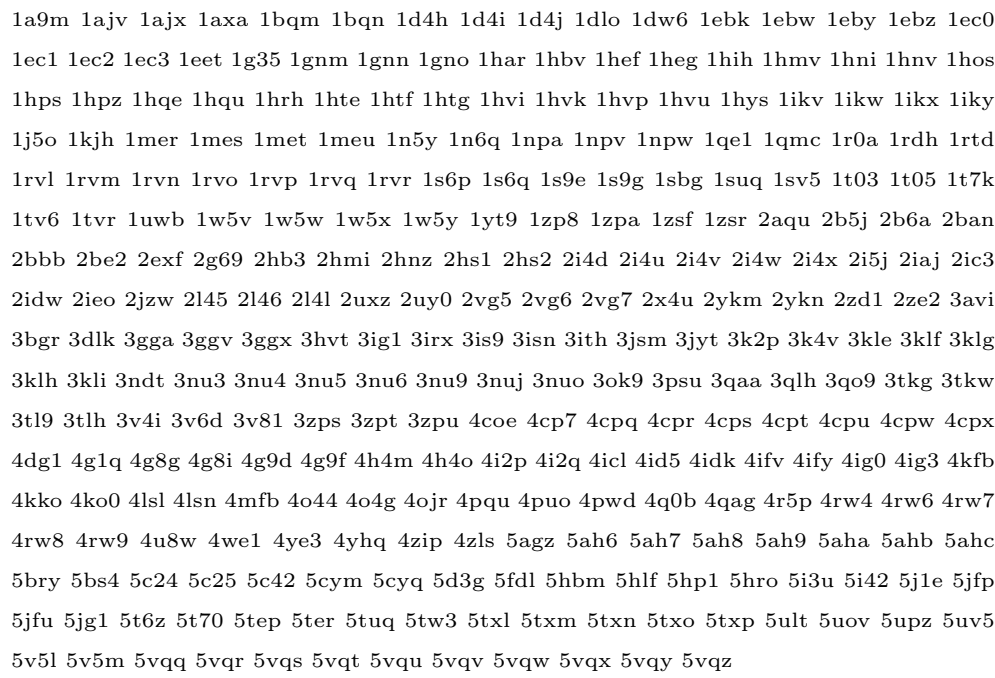 \\
\hline $\begin{array}{l}\text { mRNA of AKT1/ Target of anti- } \\
\text { sense drug/ T22306 }\end{array}$ & $\begin{array}{l}\text { 1h10 1unp 1unq 1unr 2uvm 2uzr 2uzs 3cqu 3cqw 3mv5 3mvh 3o96 3ocb 3ow4 3qkk 3qkl } \\
3 \mathrm{qkm} 4 \text { ejn } 4 \text { ekk } 4 \text { ekl } 4 \mathrm{gv} 15 \mathrm{kcv}\end{array}$ \\
\hline
\end{tabular}




\begin{tabular}{|c|c|}
\hline $\begin{array}{l}\text { 5-methyltetrahydrofolate- } \\
\text { homocysteine methyltransferase/ } \\
\text { Methyltransferase superfamily/ } \\
\text { T21661 }\end{array}$ & $2 \mathrm{o} 2 \mathrm{k} 4 \mathrm{ccz}$ \\
\hline $\begin{array}{l}\text { Phosphodiesterase (PDE) 3B/ } \\
\text { Phosphoric diester hydrolases/ } \\
\text { T21357 }\end{array}$ & $1 \mathrm{so} 21 \mathrm{soj}$ \\
\hline $\begin{array}{l}\text { Troponin I, cardiac muscle/ Tro- } \\
\text { ponin/ T20186 }\end{array}$ & 1j1d 1j1e 1lxf $1 \mathrm{mxl} 1 \mathrm{ozs} 2 \mathrm{kgb} 2 \mathrm{krd} 211 \mathrm{r} 2 \mathrm{mzp} 2 \mathrm{n} 7 \mathrm{l} 4 \mathrm{y} 99$ 5vln $5 \mathrm{w} 885 \mathrm{wcl}$ \\
\hline Cytochrome P450 2C9/ T19244 & $1 \log 2 \log 5$ 1r9o $4 \mathrm{nz} 25 \mathrm{a} 5 \mathrm{i} 5 \mathrm{a} 5 \mathrm{j} 5 \mathrm{k} 7 \mathrm{k} 5 \mathrm{w} 0 \mathrm{c} 5 \mathrm{x} 235 \mathrm{x} 245 \mathrm{xxi}$ \\
\hline $\begin{array}{l}\text { Ribosome-inactivating protein } \\
\text { alpha-trichosanthin/ T18416 }\end{array}$ & 1gis 1 giu $1 \mathrm{j} 4 \mathrm{~g} 1 \mathrm{mrj} 1 \mathrm{mrk}$ 1nli $1 \mathrm{qd} 2$ 1tcs $2 \mathrm{jdl} 2 \mathrm{jjr} 2 \mathrm{vs} 6$ \\
\hline $\begin{array}{l}\text { poly (ADP-ribose) polymerase } 3 / \\
\text { T17228 }\end{array}$ & 2eoc $3 \mathrm{c} 49$ 3c4h $3 \mathrm{ce} 03 \mathrm{fhb} 4 \mathrm{gv0} 4 \mathrm{gv} 24 \mathrm{gv} 4416 \mathrm{z} 41704171417 \mathrm{n} 417 \mathrm{o} 417 \mathrm{p} 417 \mathrm{r} 417 \mathrm{u}$ \\
\hline $\begin{array}{l}\text { mRNA of TNF alpha ligand/ Cy- } \\
\text { tokine: tumor necrosis factor/ } \\
\text { T16486 }\end{array}$ & $\begin{array}{l}1 \mathrm{a} 8 \mathrm{~m} 1 \text { tnf } 2 \mathrm{az} 52 \mathrm{e} 7 \mathrm{a} 2 \text { tun } 2 \mathrm{zjc} 2 \mathrm{zpx} 3 \mathrm{alq} 3 \mathrm{it} 8319 \mathrm{j} 3 \mathrm{wd} 54 \mathrm{~g} 3 \mathrm{y} 4 \text { tsv } 4 \text { twt } 4 \mathrm{y} 6 \text { o } 5 \mathrm{~m} 2 \mathrm{i} 5 \mathrm{~m} 2 \mathrm{j} \\
5 \mathrm{~m} 2 \mathrm{~m} 5 \mathrm{mu} 85 \text { tsw } 5 \text { uui } 5 \text { wux }\end{array}$ \\
\hline $\begin{array}{l}\text { bromodomain adjacent to zinc fin- } \\
\text { ger domain, } 2 \mathrm{~B} / \mathrm{T} 15745\end{array}$ & 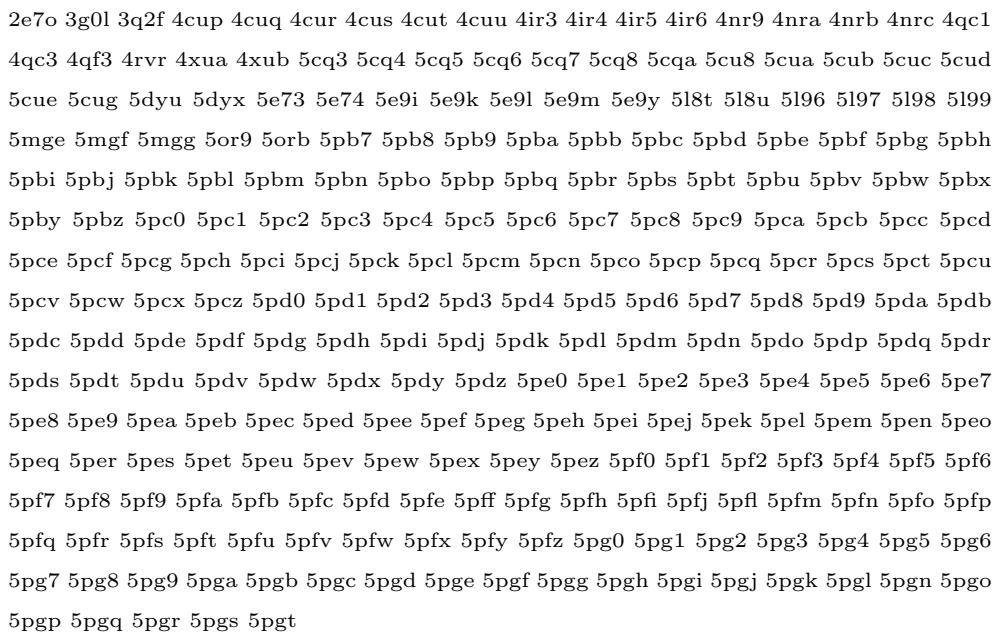 \\
\hline
\end{tabular}



1hps 1 hpz 1 hqe 1 hqu 1 hrh 1 hte 1 htf 1 htg 1 hvi 1 hvk 1 hvp 1 hvu 1 hys 1 ikv 1 ikw 1 ikx 1 iky $1 \mathrm{j} 5$ o $1 \mathrm{kjh} 1 \mathrm{mer} 1 \mathrm{mes} 1 \mathrm{met} 1 \mathrm{meu} 1 \mathrm{n} 5 \mathrm{y} 1 \mathrm{n} 6 \mathrm{q}$ 1npa $1 \mathrm{npv} 1 \mathrm{npw} 1 \mathrm{qe} 1$ 1qme $1 \mathrm{r} 0 \mathrm{a} 1 \mathrm{rdh} 1 \mathrm{rtd}$ 1rvl 1rvm 1rvn 1rvo 1rvp 1rvq 1rvr 1s6p 1s6q 1s9e 1s9g 1sbg 1suq 1sv5 1t03 1t05 1t7k 1tv6 1tvr 1uwb 1w5v 1w5w 1w5x 1w5y 1yt9 1zp8 1zpa 1zsf 1zsr 2aqu 2b5j 2b6a 2ban 2bbb 2be2 2exf 2g69 2hb3 2hmi 2hnz 2hs1 2hs2 2i4d 2i4u 2i4v 2i4w 2i4x 2i5j 2iaj 2ic3 2idw 2ieo 2jzw 214521462141 2uxz 2uy0 2vg5 2vg6 2vg7 2x4u 2ykm 2ykn 2zd1 2ze2 3avi 3bgr 3dlk 3gga 3ggv 3ggx 3hvt 3ig1 3irx 3is9 3isn 3ith 3jsm 3jyt 3k2p 3k4v 3kle 3klf 3klg 3klh 3kli 3ndt 3nu3 3nu4 3nu5 3nu6 3nu9 3nuj 3nuo 3ok9 3psu 3qaa 3qlh 3qo9 3tkg 3tkw 3t19 3tlh 3v4i 3v6d 3v81 3zps 3zpt 3zpu 4coe 4cp7 4cpq 4cpr 4cps 4cpt 4cpu 4cpw 4cpx 4dg1 4g1q 4g8g 4g8i 4g9d 4g9f 4h4m 4h4o 4i2p 4i2q 4icl 4id5 4idk 4ifv 4ify 4ig0 4ig3 4kfb 4 kko $4 \mathrm{ko0} 4 \mathrm{lsl} 4 \mathrm{lsn} 4 \mathrm{mfb} 4 \mathrm{o} 44$ 4o4g 4ojr 4pqu 4puo 4pwd 4q0b 4qag 4r5p 4rw4 4rw6 4rw7 4rw8 4rw9 4u8w 4we1 4ye3 4yhq 4zip 4zls 5agz 5ah6 5ah7 5ah8 5ah9 5aha 5ahb 5ahc 5 bry 5 bs 4 5c24 5c25 5c42 5cym 5cyq 5d3g 5fdl 5hbm 5hlf 5hp1 5hro 5i3u 5i42 5j1e 5jfp 5 jfu 5 jg1 5t6z 5t70 5tep 5ter 5tuq 5tw3 5txl 5txm 5txn 5txo 5txp 5ult 5uov 5upz 5uv5 $5 \mathrm{v} 515 \mathrm{v} 5 \mathrm{~m} 5 \mathrm{vqq} 5 \mathrm{vqr} 5 \mathrm{vqs} 5 \mathrm{vqt} 5 \mathrm{vqu} 5 \mathrm{vqv} 5 \mathrm{vqw} 5 \mathrm{vqx} 5 \mathrm{vqy} 5 \mathrm{vqz}$ Short-chain dehydrogenases reductases/ T15139

HIV p7 nucleocapsid protein/ $\mathrm{T} 15107$
Phosphoglycerate dehydrogenase/

2 g76 5n53 5nzo 5nzp 5nzq 5ofm 5ofv 5ofw

1a9m 1ajv 1ajx 1axa 1bqm 1bqn 1d4h 1d4i 1d4j 1dlo 1dw6 1ebk 1ebw 1eby 1ebz 1ec0 1ec1 1ec2 1ec3 1eet 1g35 1gnm 1gnn 1gno 1har 1hbv 1 hef 1 heg 1 hih $1 \mathrm{hmv} 1 \mathrm{hni} 1 \mathrm{hnv} 1 \mathrm{hos}$ $1 \mathrm{hps} 1 \mathrm{hpz} 1 \mathrm{hqe} 1 \mathrm{hqu} 1 \mathrm{hrh} 1 \mathrm{hte} 1 \mathrm{htf} 1 \mathrm{htg} 1 \mathrm{hvi} 1 \mathrm{hvk} 1 \mathrm{hvp} 1 \mathrm{hvu} 1 \mathrm{hys} 1 \mathrm{ikv} 1 \mathrm{ikw} 1 \mathrm{ikx} 1 \mathrm{iky}$ $1 \mathrm{j} 5 \mathrm{o} 1 \mathrm{kjh} 1 \mathrm{mer} 1 \mathrm{mes} 1 \mathrm{met} 1 \mathrm{meu} 1 \mathrm{n} 5 \mathrm{y}$ 1n6q 1npa $1 \mathrm{npv} 1 \mathrm{npw} 1 \mathrm{qe} 1$ 1qme $1 \mathrm{r} 0 \mathrm{a} 1 \mathrm{rdh} 1 \mathrm{rtd}$ $1 \mathrm{rvl} 1 \mathrm{rvm}$ 1rvn 1rvo 1rvp 1rvq 1rvr 1s6p 1s6q 1s9e 1s9g 1sbg 1suq 1sv5 1t03 1t05 1t7k 1tv6 1tvr 1uwb 1w5v 1w5w 1w5x 1w5y 1yt9 1zp8 1zpa 1zsf 1zsr 2aqu 2b5j 2b6a 2ban 2bbb 2be2 2exf 2g69 2hb3 2hmi 2hnz 2hs1 2hs2 2i4d 2i4u 2i4v 2i4w 2i4x 2i5j 2iaj 2ic3 2idw 2ieo 2jzw 214521462141 2uxz 2uy0 2vg5 2vg6 2vg7 2x4u 2ykm 2ykn 2zd1 2ze2 3avi 3bgr 3dlk 3gga 3ggv 3ggx 3hvt 3ig1 3irx 3is9 3isn 3ith 3jsm 3jyt 3k2p 3k4v 3kle 3klf 3klg 3klh 3kli 3ndt 3nu3 3nu4 3nu5 3nu6 3nu9 3nuj 3nuo 3ok9 3psu 3qaa 3qlh 3qo9 3tkg 3tkw 3t19 3tlh 3v4i 3v6d 3v81 3zps 3zpt 3zpu 4coe 4cp7 4cpq 4cpr 4cps 4cpt 4cpu 4cpw 4cpx 4dg1 4g1q 4g8g 4g8i 4g9d 4g9f 4h4m 4h4o 4i2p 4i2q 4icl 4id5 4idk 4ifv 4ify 4ig0 4ig3 4kfb 4kko 4ko0 4lsl 4lsn 4mfb 4o44 4o4g 4ojr 4pqu 4puo 4pwd 4q0b 4qag 4r5p 4rw4 4rw6 4rw7 4rw8 4rw9 4u8w 4we1 4ye3 4yhq 4zip 4zls 5agz 5ah6 5ah7 5ah8 5ah9 5aha 5ahb 5ahc 5bry 5bs4 5c24 5c25 5c42 5cym 5cyq 5d3g 5fdl 5hbm 5hlf 5hp1 5hro 5i3u 5i42 5j1e 5jfp 5 jfu 5 jg1 5 t6z 5 t70 5tep 5ter 5tuq 5tw3 5 txl 5txm 5txn 5txo 5txp 5ult 5uov 5upz 5uv5 $5 \mathrm{v} 515 \mathrm{v} 5 \mathrm{~m} 5 \mathrm{vqq} 5 \mathrm{vqr} 5 \mathrm{vqs} 5 \mathrm{vqt} 5 \mathrm{vqu} 5 \mathrm{vqv} 5 \mathrm{vqw} 5 \mathrm{vqx} 5 \mathrm{vqy} 5 \mathrm{vqz}$

Lactate dehydrogenase A/ Short1i10 4ajp 4jnk 414r 4l4s 4m49 4ojn 4okn 4qo7 4qo8 4qsm 4qt0 4r68 4r69 4rls 4zvv 5ixs 5 ixy

chain dehydrogenases reductases/ $\mathrm{T} 15053$

1flt 1qsv 1qsz 1qty 1rv6 2xac 3hng 4ckv 4cl7 5abd 5ex3 5t89
mRNA of VEGF-1 receptor/

T14439 


\begin{tabular}{|c|c|}
\hline $\begin{array}{l}\text { Ras-related protein Rab-7a/ Small } \\
\text { GTPase superfamily/ T14030 }\end{array}$ & 1t91 1yhn 3law \\
\hline $\begin{array}{l}\text { Liver receptor homolog-1/ Nuclear } \\
\text { hormone receptor/ T13902 }\end{array}$ & 1yok 1yuc 1zdu 2a66 3plz 3tx7 4dor 4dos 4is8 4oni 4pld 4ple 4rwv 510m $51115 \mathrm{syz} 5$ unj \\
\hline $\begin{array}{l}\text { eukaryotic translationinitiation fac- } \\
\text { tor 2-alpha kinase } 3 / \mathrm{T} 13176\end{array}$ & $4 \mathrm{~g} 314 \mathrm{~g} 344 \mathrm{~m} 7 \mathrm{i} 4 \mathrm{x} 7 \mathrm{~h} 4 \mathrm{x} 7 \mathrm{j} 4 \mathrm{x} 7 \mathrm{k} 4 \mathrm{x} 7 \mathrm{l} 4 \mathrm{x} 7 \mathrm{n} 4 \mathrm{x} 7 \mathrm{o} 4 \mathrm{yzs} 5 \mathrm{sv} 7$ \\
\hline $\begin{array}{l}\text { Farnesyl protein transferase/ } \\
\text { T13127 }\end{array}$ & 1jcq 1ld7 1ld 8 1mzc 1s63 1sa4 1tn6 2f0y 2h6f 2h6g 2h6h 2h6i 2iej 3e37 \\
\hline activin A receptor, type I/ T12091 & 3h9r 3mtf 3oom 3q4u 4bgg 4c02 4dym 5oxg 5oy6 6eix \\
\hline $\begin{array}{l}\text { Cytochrome P450 2B6/ Oxidore- } \\
\text { ductases acting on paired donors/ } \\
\text { T11793 }\end{array}$ & 3ibd 3qoa 3qu8 3ua5 4i91 4rql 4rrt 4zv8 5uap 5uda 5uec 5ufg \\
\hline mRNA of WEE1/ Kinase/ T10939 & 1x8b 2in6 2io6 2z2w 3bi6 3biz 3cqe $3 \mathrm{cr} 05 \mathrm{v} 5 \mathrm{y} 5 \mathrm{vc} 35 \mathrm{vc} 45 \mathrm{vc} 5 \quad 5 \mathrm{vc} 6$ 5vd2 \\
\hline $\begin{array}{l}\text { mRNA of HCV core protein/ } \\
\text { T09874 }\end{array}$ & 1rtl 2a4g 2gvf 3eyd 3hkw 3kn2 3qgh 3qgi 3rc4 3rc5 3su4 \\
\hline $\begin{array}{l}\text { Retinol dehydrogenase/ Short- } \\
\text { chain dehydrogenases reductases/ } \\
\text { T09770 }\end{array}$ & $\operatorname{lagn} 1 \mathrm{~d} 1 \mathrm{~s} 1 \mathrm{~d} 1 \mathrm{t}$ \\
\hline $\begin{array}{l}\text { Cyclin dependent kinase } 5 \text { activator } \\
1 / \text { Cyclin-dependent kinase } 5 \text { acti- } \\
\text { vator/ T09513 }\end{array}$ & 1h4l 1ung 1unh 1unl 3o0g \\
\hline $\begin{array}{l}\text { discoidin domain receptor tyrosine } \\
\text { kinase } 1 / \text { T09185 }\end{array}$ & 3zos 4 ag 4 bbj 4 ckr 5 bvk 5 bvn 5 bvo 5 bvw 5 fdp $5 \mathrm{fdx}$ \\
\hline Caspase-2/ Peptidase/ T09128 & 1pyo 2p2c 3r5j 3r6g 3r61 3r7b 3r7n 3r7s 3rjm \\
\hline $\begin{array}{l}\text { DTDP-4-dehydrorhamnose } 3,5- \\
\text { epimerase rmlC/ Racemases and } \\
\text { epimerases/ T08218 }\end{array}$ & 1pm7 1upi 2ixc \\
\hline $\begin{array}{l}\text { oxidative stress responsive } 1 / \\
\text { T08198 }\end{array}$ & 2v3s 2vwi 3dak \\
\hline Integrin alpha-1/ beta-1/ T06717 & 1k11 1lha 3g9w 3t9k 3vi3 3vi4 4dx9 4wjk 4wk0 4wk2 4wk4 \\
\hline $\begin{array}{l}\text { Hepatitis C virus NS5B poly- } \\
\text { merase/ T05152 }\end{array}$ & 1rtl 2a4g 2gvf 3eyd 3hkw 3kn2 3qgh 3qgi 3rc4 3rc5 3su4 \\
\hline
\end{tabular}




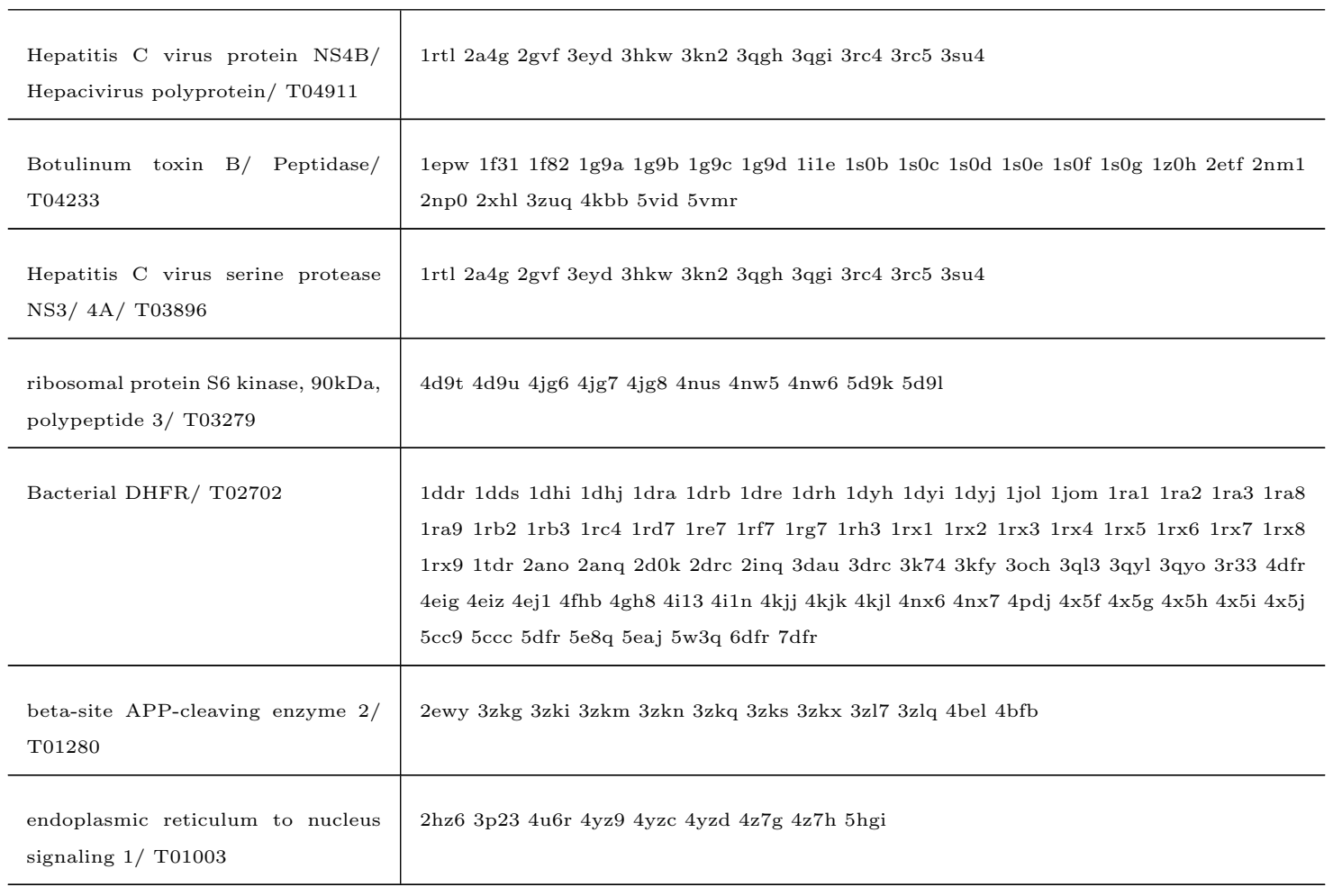


Table S3, related to Chapter 3. Benchmark for validation

\begin{tabular}{|c|c|c|}
\hline Drug Name & $\begin{array}{l}\text { number of prediction pdbs } \\
\text { ranked in top } 10 \%\end{array}$ & number of true positive pdbs \\
\hline Dorzolamide & 2 & 3 \\
\hline Lovastatin & 2 & 3 \\
\hline Estradiol & 2 & 3 \\
\hline Efavirenz & 1 & 1 \\
\hline Delavirdine & 1 & 1 \\
\hline Niflumic acid & 0 & 1 \\
\hline Nevirapine & 1 & 1 \\
\hline Galantamine & 1 & 1 \\
\hline Sitagliptin & 1 & 1 \\
\hline Tadalafil & 3 & 3 \\
\hline Imatinib & 3 & 3 \\
\hline Succinylcholine & 1 & 1 \\
\hline Apixaban & 1 & 1 \\
\hline Cocaine & 0 & 1 \\
\hline Dichlorphenamide & 1 & 3 \\
\hline Proflavine & 0 & 1 \\
\hline Minocycline & 1 & 1 \\
\hline Indomethacine & 3 & 6 \\
\hline Pentoxifylline & 0 & 8 \\
\hline Chloramphenicol & 1 & 9 \\
\hline Topiramate & 2 & 3 \\
\hline Papaverine & 0 & 7 \\
\hline
\end{tabular}




\begin{tabular}{|c|c|c|}
\hline Balsalazide & 1 & 2 \\
\hline Ethoxzolamide & 0 & 3 \\
\hline Gefitinib & 3 & 20 \\
\hline Prazosin & 1 & 2 \\
\hline Pyrimethamine & 1 & 2 \\
\hline Ethacrynic acid & 1 & 1 \\
\hline Abacavir & 0 & 1 \\
\hline Captopril & 2 & 3 \\
\hline Celecoxib & 2 & 2 \\
\hline Levonorgestrel & 4 & 5 \\
\hline Zonisamide & 1 & 4 \\
\hline Ampicillin & 0 & 1 \\
\hline Progesterone & 4 & 5 \\
\hline Diazepam & 0 & 1 \\
\hline Diclofenac & 1 & 2 \\
\hline Methotrexate & 2 & 2 \\
\hline Clomipramine & 1 & 2 \\
\hline Trimethoprim & 2 & 2 \\
\hline Dexamethasome & 1 & 2 \\
\hline Hydrocortisone & 2 & 2 \\
\hline Fludrocortisone & 3 & 3 \\
\hline Trifluoperazine & 1 & 4 \\
\hline Hydrochlorothiazide & 1 & 3 \\
\hline Sildenafil & 3 & 3 \\
\hline Ursodeoxycholic & 2 & 2 \\
\hline
\end{tabular}



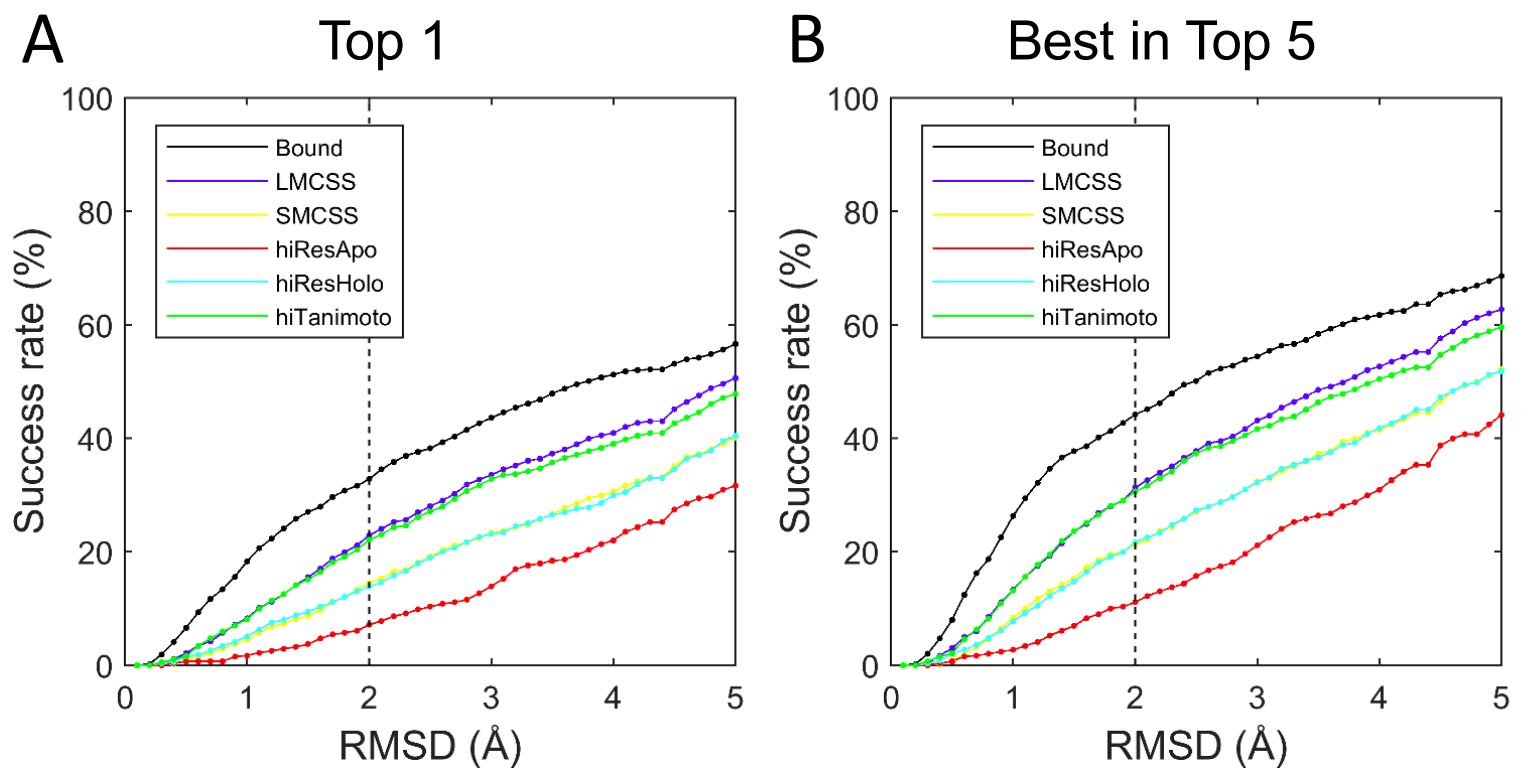

Figure S2, related to Figure 4.1. The results of the binding mode prediction based on the Vina Score. (A) The success rate when only the top predicted model was considered for each protein structure. When $2.0 \AA$ was used as the threshold, the success rates of dockings with the bound, LMCSS, SMCSS, hiResHolo, hiResApo, and hiTanimoto protein structures are $32.8 \%, 22.9 \%, 14.5 \%, 13.8 \%, 7.1 \%, 22.1 \%$, respectively. (B) The success rate when top 5 models were considered for each protein structure. When $2.0 \AA$ was used as the threshold, the success rates of dockings with the bound, LMCSS, SMCSS, hiResHolo, hiResApo, and hiTanimoto protein structures were $44.2 \%, 31.3 \%, 21.3 \%, 21.7 \%, 11.1 \%$, and $30.6 \%$, respectively. 
Table S4, related to Table 4.1. The results of binding mode prediction for the CELPP targets based on the Vina Score. The first row (Bound) lists the results of docking with the bound protein structures. The last row (hiSHAFTS) gives the results of docking with the user-specified protein structures, and the other five rows show the results of docking using the candidate protein structures provided by CELPP. The error of each value was estimated with the bootstrap method, in which 1000 replicates were used, and was reported in the parentheses.

\begin{tabular}{ccccccccc}
\hline & & \multicolumn{3}{c}{ Top 1 } & \multicolumn{3}{c}{ Best in Top 5 } & \multicolumn{2}{c}{ Best in All } \\
\cline { 3 - 8 } $\begin{array}{c}\text { Protein } \\
\text { Structure }\end{array}$ & $\begin{array}{c}\text { Number } \\
\text { of } \\
\text { Targets }\end{array}$ & $\begin{array}{c}\text { Median } \\
\text { RMSD } \\
(\AA)\end{array}$ & $\begin{array}{c}\text { Median } \\
\text { RMSD } \\
(\AA)\end{array}$ & $\begin{array}{c}\text { Median } \\
\text { RMSD } \\
(\AA)\end{array}$ & $\begin{array}{c}\text { Median } \\
\text { RMSD } \\
(\AA)\end{array}$ & $\begin{array}{c}\text { Median } \\
\text { RMSD } \\
(\AA)\end{array}$ & $\begin{array}{c}\text { Median } \\
\text { RMSD } \\
(\AA)\end{array}$ \\
\hline Bound & 969 & $3.9(0.3)$ & $4.8(0.1)$ & $2.5(0.2)$ & $3.7(0.1)$ & $1.2(0.0)$ & $1.5(0.0)$ \\
LMCSS & 969 & $5.0(0.2)$ & $5.3(0.1)$ & $3.8(0.2)$ & $4.3(0.1)$ & $1.5(0.0)$ & $1.9(0.0)$ \\
SMCSS & 969 & $6.0(0.1)$ & $5.9(0.1)$ & $4.8(0.2)$ & $5.1(0.1)$ & $1.9(0.1)$ & $2.4(0.1)$ \\
hiResApo & 608 & $6.5(0.1)$ & $6.7(0.1)$ & $5.6(0.2)$ & $5.7(0.1)$ & $2.2(0.1)$ & $2.8(0.1)$ \\
hiResHolo & 969 & $5.8(0.2)$ & $6.0(0.1)$ & $4.8(0.2)$ & $5.0(0.1)$ & $1.8(0.1)$ & $2.4(0.1)$ \\
hiTanimoto & 969 & $5.2(0.1)$ & $5.4(0.1)$ & $4.0(0.2)$ & $4.0(0.1)$ & $1.5(0.0)$ & $1.9(0.0)$ \\
hiSHAFTS & 875 & $5.2(0.2)$ & $5.5(0.1)$ & $4.0(0.2)$ & $4.5(0.1)$ & $1.6(0.1)$ & $1.9(0.0)$ \\
\hline
\end{tabular}




\section{Bibliography}

[1] TheUniProtConsortium, Nucleic Acids Research 2016, 45, D158-D169.

[2] Berman, H. M. Nucleic Acids Research 2000, 28, 235-242.

[3] Li, Y. H. et al. Nucleic acids research 2017, 46, D1121-D1127.

[4] Yan, C.; Zou, X. Computer-Aided Drug Discovery; Springer, 2015; pp 153-166.

[5] Huang, S.-Y.; Zou, X. Journal of Computational Chemistry 2006, 27, 1866-1875.

[6] Yan, C.; Grinter, S. Z.; Merideth, B. R.; Ma, Z.; Zou, X. Journal of Chemical Information and Modeling 2015, 56, $1013-1021$. 


\section{VITA}

Zhiwei Ma was born in June 1987 in Hailun, Heilongjiang Province, China. She obtained her Bachelor of Science degree in Physics from Taiyuan Normal University in June, 2010. She obtained her Master of Science degree in Physics from Hangzhou Normal University in June, 2013.

After graduation, she was admitted into Department of Physics and Astronomy at University of Missouri as a Ph.D student under Dr. Xiaoqin Zou's supervision, and also joined the Zou Lab as a research assistant in 2014. During the PhD period, she conducted research in the field of computational structure biology. She focused on developing the large scale molecular docking methods to predict protein-ligand interactions. With the completion of her PhD study, she will join the Dr. TzyhChang Hwang's lab for her postdoctoral research. 